The extraction of the spin structure function, g2 (and g1) at low Bjorken $\mathrm{x}$

\title{
By
}

Luwani Zurmbonwi Ndukum

\author{
A Dissertation \\ Submitted to the Faculty of \\ Mississippi State University \\ in Partial Fulfillment of the Requirements \\ for the Degree of Doctor of Philosophy \\ in Applied Physics \\ in the Bagley College of Engineering
}

Mississippi State, Mississippi

August 2015 


\section{Copyright by}

\section{Luwani Zurmbonwi Ndukum}


The extraction of the spin structure function, g2 (and g1) at low Bjorken $\mathrm{x}$

By

Luwani Zurmbonwi Ndukum

Approved:

James A. Dunne
(Major Professor)

Dipangkar Dutta

(Committee Member)

Oscar A. Rondon-Aramayo

(Committee Member)

Radhakrishnan Srinivasan

(Committee Member)

Gautam Rupak Lan Tai Moong

(Committee Member)

Hendrik F. Arnoldus

(Graduate Coordinator)

Jason Keith
Interim Dean
Bagley College of Engineering


Name: Luwani Zurmbonwi Ndukum

Date of Degree: August 14, 2015

Institution: Mississippi State University

Major Field: Applied Physics

Major Professor: Dr. James A. Dunne

Title of Study: The extraction of the spin structure function, g2 (and g1) at low Bjorken $\mathrm{X}$

Pages of Study: 161

Candidate for Degree of Doctor of Philosophy

The Spin Asymmetries of the Nucleon Experiment (SANE) used the Continuous Electron Beam Accelerator Facility at Jefferson Laboratory in Newport News, VA to investigate the spin structure of the proton. The experiment measured inclusive double polarization electron asymmetries using a polarized electron beam, scattered off a solid polarized ammonia target with target polarization aligned longitudinal and near transverse to the electron beam, allowing the extraction of the spin asymmetries A1 and A2, and spin structure functions g1 and g2. Polarized electrons of energies of 4.7 and $5.9 \mathrm{GeV}$ were used. The scattered electrons were detected by a novel, non-magnetic array of detectors observing a four-momentum transfer range of 2.5 to $6.5 \mathrm{GeV}^{*} \mathrm{~V}$. This document addresses the extraction of the spin asymmetries and spin structure functions, with a focus on spin structure function, g2 (and g1) at low Bjorken $\mathrm{x}$. The spin structure functions were measured as a function of $\mathrm{x}$ and $\mathrm{W}$ in four $\mathrm{Q}$ square bins. A full understanding of the low $\mathrm{x}$ region is 
necessary to get clean results for SANE and extend our understanding of the kinematic region at low $\mathrm{x}$. 


\section{DEDICATION}

To my mother, Teresa Siri Ndukum. 


\section{ACKNOWLEDGEMENTS}

This material is based upon work supported by the U.S. Department of Energy, Office of Science, Office of Nuclear Physics under contract DE-AC05-06OR23177.

I thank my committee members for their comments on this dissertation, and I thank James Dunne my advisor for giving me such a great opportunity and for the support all the way. I have learned a great deal doing research at Jefferson Lab (JLab).

I want to take this opportunity to say a special thank you to all the members of the SANE analysis collaboration. Most importantly I want to appreciate Oscar Rondon for his support and patience in guiding me through the SANE analysis. Furthermore, I can say I was very lucky to have Mark Jones as my JLab supervisor, Mark was always ready to provide answers to my questions, make valuable comments and contribution to my work, there was always that sense of joy when you leave Mark's office because you know that he will always create time to attend to you.

I equally want to thank my family and friends for their love and support through out this journey. My gratitude to Juliet Ndukum for her financial support and advises through out graduate school. Tchefor Ndukum inspired me to continue in Physics. Not forgetting Mr Crispus Mbenga, my secondary school physics teacher who was very instrumental in making me fall in love with Physics, I say a big thank you. 
Most importantly, I want to thank my wife, Elisabeth Ndukum for her unwavering support and understanding during the writing of this dissertation. 


\section{TABLE OF CONTENTS}

DEDICATION $\ldots \ldots \ldots \ldots \ldots \ldots \ldots \ldots \ldots \ldots \ldots \ldots \ldots \ldots \ldots$

ACKNOWLEDGEMENTS . . . . . . . . . . . . . . . . . iii

LIST OF TABLES $\ldots \ldots \ldots \ldots \ldots \ldots$ viii

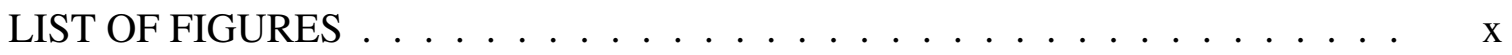

\section{CHAPTER}

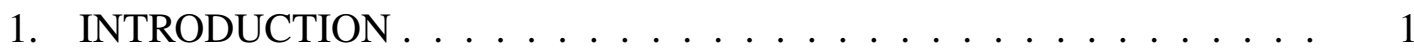

$1.1 \quad$ Lepton Scattering . . . . . . . . . . . . . . . . . 3

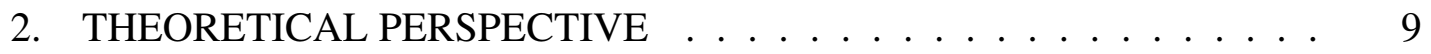

$2.1 \quad$ Investigating the Internal Structure of the Proton $\ldots \ldots \ldots$

2.2 Kinematics and Variable . . . . . . . . . . . . . . . . . 10

2.3 Electromagnetic Interaction . . . . . . . . . . . . . . 11

2.4 Elastic Scattering (Electron-Nucleon) _ . . . . . . . . . . 11

2.5 Deep Inelastic Scattering and The Parton Model . . . . . . . . . 17

2.6 Physics of Parton Distribution . . . . . . . . . . . . . 20

2.7 Quark Spin Structure of the Nucleon . . . . . . . . . . . . . 24

2.8 Moments and Twist . . . . . . . . . . . . . . . 27

3. EXPERIMENTAL SETUP . . . . . . . . . . . . . . . . . 33

$3.1 \quad$ Experimental Apparatus $\ldots \ldots \ldots \ldots . \ldots \ldots$

3.2 The Accelerator . . . . . . . . . . . . . . . . . . 34

3.2.1 The Polarized Beam Source . . . . . . . . . . . . . 36

3.2.2 The Polarized Electron Production . . . . . . . . . 36

3.2.3 Acceleration and Delivery of Beam . . . . . . . . . 38

3.2.4 Beam Current Measurement . . . . . . . . . . . . . . . 39

3.2.5 Beam Energy Measurement . . . . . . . . . . . . 40

3.2.6 Beam Position Measurement . . . . . . . . . . . 40 
3.2.7 Beam Polarization Measurement . . . . . . . . . . . . 41

3.2.8 The Raster System . . . . . . . . . . . . . . . . . . . 46

3.3 Chicane and Helium Bag . . . . . . . . . . . . . . . . . 47

3.4 Target . . . . . . . . . . . . . . . . . 49

$3.4 .1 \quad$ Why Ammonia . . . . . . . . . . . . . . . 49

3.4.2 Dynamic Nuclear Polarization DNP . . . . . . . . . 49

3.4.3 Thermal Equilibrium Polarization $\ldots \ldots \ldots \ldots$. . . . 50

3.4.4 Solid-State Effect . . . . . . . . . . . . . . . . 51

3.4.5 Equal Spin Temperature Theory . . . . . . . . . . 53

3.4 .6 Target Cryogenic . . . . . . . . . . . . . . 55

3.4 .7 Microwaves . . . . . . . . . . . . . . 58

3.4.8 Nuclear Magnetic Resonance and Polarization . . . . . . 60

3.4.9 Radiation Damage and Lifespan of a Target Load . . . . . 63

3.5 Electron Detector Package . . . . . . . . . . . . . . 65

3.5.1 The BigCal Electromagnetic Calorimeter . . . . . . . . 67

3.5.2 Čerenkov Detector . . . . . . . . . . . . . . 68

3.5.3 Hodoscopes . . . . . . . . . . . . . . 70

3.5.3.1 Lucite Hodoscope . . . . . . . . . . . . 70

3.5.3.2 Forward (Front) Tracker . . . . . . . . . . . 71

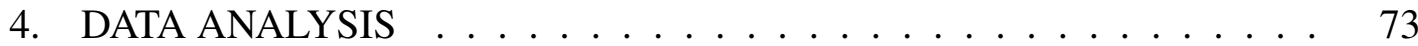

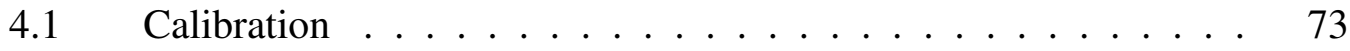

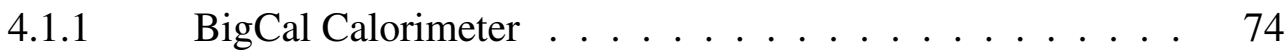

4.2 Artificial Neural Network _. . . . . . . . . . . . . . 75

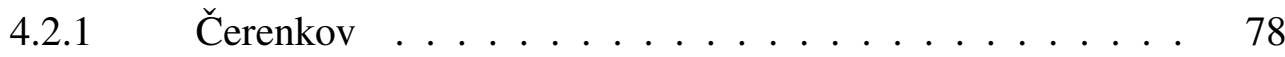

4.3 Event Reconstruction and Selection _ . . . . . . . . . . . 79

4.3.1 Event Criteria . . . . . . . . . . . . . . 82

4.4 Asymmetries and Structure Functions Analysis . . . . . . . . 83

4.4.1 Charge Normalization . . . . . . . . . . . . . . . . 84

4.4.2 Livetime Correction . . . . . . . . . . . . . . . . 84

4.4.3 Dilution Factor and Packing Fractions . . . . . . . . 85

4.4.4 Radiative Corrections . . . . . . . . . . . . . . . 90

4.5 Structure Function from Measurable Asymmetries and Cross-Section 92

4.6 Extraction of Asymmetries and Structure Functions . . . . . . . 94

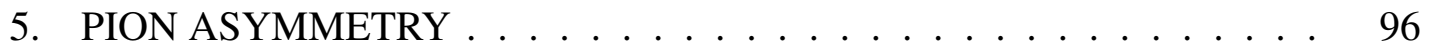

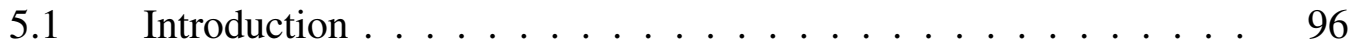

5.2 Pair-Sysmetric Background . . . . . . . . . . . . . 98

5.2.1 Double Track Background from the Tracker . . . . . . . 99

5.3 Trajectory of a $e^{+} e^{-}$pair in SANE . . . . . . . . . . 100

5.4 Calculating the Pair-Symmetric Background Asymmetry . . . . . 100 
5.5 Systematic Error in Background Correction . . . . . . . . . . . 112

$5.6 \quad$ Calculating $A_{b}$ and $f_{b} \ldots \ldots \ldots \ldots \ldots \ldots$

5.6.1 $A_{b}$ for SANE's $A_{180^{\circ}}$ Configuration . . . . . . . 117

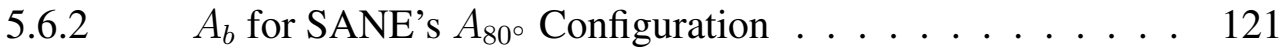

$5.6 .3 \quad$ Calculating $f_{b} \ldots \ldots \ldots \ldots \ldots \ldots$

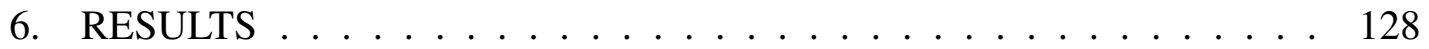

$6.1 \quad$ Results of $A_{180}$ and $A_{80} \quad \ldots \ldots \ldots \ldots \ldots$

6.2 Results of the Spin Asymmetries . . . . . . . . . . . . . . . . . 129

6.3 Results of the Spin Structure Functions . . . . . . . . . . . . . . 129

6.4 Background Corrections . . . . . . . . . . . . . . 136

7. CONCLUSIONS ..................... 141

$7.1 \quad$ Conclusion . . . . . . . . . . . . . . . . . 141

\section{APPENDIX}

A. BEAM ENERGY COMBINATIONS FOR EXPERIMENTAL HALLS AND $\pi^{0}$ ASYMMETRY DATA FOR ALL RUNS $\ldots \ldots \ldots$. . . . . . . . 148

A.1 Beam Energy Combinations for Experimental Halls . . . . . . . . 149

A.2 $\pi^{0}$ Asymmetry Data for All Runs _ . . . . . . . . . . . 152 


\section{LIST OF TABLES}

3.1 Beam Energies per Run for the various SANE run periods . . . . . . . . . 41

$3.2 \quad$ Chicane Setting . . . . . . . . . . . . . . . . 48

4.1 Good Runs with the Energy and Target Field Angle . . . . . . . . . . . . 83

4.2 Material thickness that contributed to radiative processes . . . . . . . . . 92

5.1 Total Average Asymmetry for the Various Energy Cuts . . . . . . . . . 110

5.2 Pion Asymmetries for the Various Energy and Angle Orientations . . . . . 112

5.3 $\pi^{0}$ Asymmetries Combined by Groups of Runs with the Same Sign of the Product of $P_{b} * P_{t} \ldots \ldots \ldots \ldots \ldots \ldots$

$5.4 \quad$ SANE and SLAC experiments were at comparable $P_{T} \ldots \ldots \ldots 117$

5.5 Ratio of Background Particles to Electrons _. . . . . . . . . . . 125

5.6 Error Results and the A Numerical Example of the Corrected Asymmetry $A \quad 126$

5.7 Numerical Example to Illustrate the Corrected Asymmetries $A_{180^{\circ}}$ and $A_{80^{\circ}} \quad 127$

6.1 SANE Configuration . . . . . . . . . . . . . . . 128

A.1 $\pi^{0}$ Asymmetries for All Runs _. . . . . . . . . . . . . . 153 


\section{LIST OF FIGURES}

1.1 Plot of SANE Kinematics $\ldots \ldots \ldots \ldots \ldots$

2.1 Elastic Scattering Showing the Four-momentum $q$ of the Virtual Photon . . 10

2.2 Deep Inelastic Scattering . . . . . . . . . . . . . . . . . . . . 17

$2.3 \quad$ Plot Showing $F_{2}^{n} / F_{2}^{p}$ Ratio $\ldots \ldots \ldots \ldots \ldots \ldots \ldots \ldots \ldots$

2.4 Experimental data of the proton structure function $F_{2}^{p}(x)$ at various values

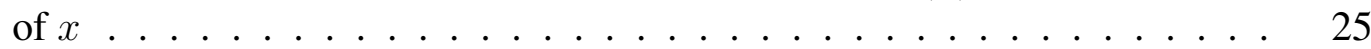

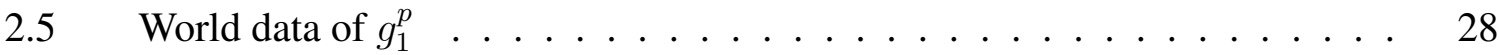

$2.6 \quad$ Data of $g_{1}$ plotted versus $x \ldots \ldots \ldots \ldots \ldots \ldots \ldots$

$2.7 \quad$ Data of $g_{2}$ plotted versus $x$, with $g_{2}^{W W} \ldots \ldots \ldots \ldots \ldots \ldots$

$2.8 \quad$ Expected SANE results for $\bar{d}_{2} \ldots \ldots \ldots \ldots \ldots \ldots \ldots$

$3.1 \quad$ Experimental Layout Showing the Various Components Involved $\ldots \ldots$

3.2 An Annotated Diagram of the CEBAF Accelerator . . . . . . . . . 36

3.3 Beam position in units of $\mathrm{mm}$ for all the run numbers . . . . . . . . . 42

$3.4 \quad$ SANE's Beam Polarization per Run number . . . . . . . . . . . . . . . . . 43

3.5 SANE Beamline During Perpendicular Target Field Running . . . . . . . . 48

3.6 Schematic Demonstration of the Solid-State Effect . . . . . . . . . . . 52

$3.7 \quad$ Population Densities of the Electron Levels $\ldots \ldots \ldots$

$3.8 \quad$ Target and magnet used for SANE $\ldots \ldots \ldots \ldots \ldots \ldots \ldots$ 
$3.9 \quad$ Target Ladder . . . . . . . . . . . . . . . . . . . . . . . . . . . . . . . 59

3.10 Schematic Drawing of the $Q$-meter (NMR) Circuit . . . . . . . . . . 61

3.11 NMR signal decomposition $\ldots \ldots \ldots$. . . . . . . . . . . 63

3.12 Charge averaged target polarization per run for SANE $\ldots \ldots \ldots$. . . . . 64

3.13 Charge averaged target polarization per run for SANE $\ldots \ldots \ldots 6$

3.14 Annotated Picture of BETA with DIS Electron Simulation . . . . . . . 66

3.15 Rear View of Lead-Glass in BigCal (Left), Trigger Logic (Right) . . . . . . 68

3.16 Picture of the front tracker . . . . . . . . . . . . . . . . . . . 72

4.1 Reconstructed $\pi^{0}$ Mass. $P_{2}=$ Gaussian Fit and $P_{3}=\sigma \ldots \ldots \ldots$

$4.2 \quad$ Neural Network schematics _. . . . . . . . . . . . . . . . . . . 77

4.3 Difference between generated and reconstructed $Y, X$, and $E \ldots \ldots$

4.4 Čerenkov ADC Showing Two Peaks . . . . . . . . . . . . . . . . . . . 80

4.5 Čerenkov TDC versus ADC values _ . . . . . . . . . . . . . . . . 81

4.6 Diagram of BETA with Physics Angles _ . . . . . . . . . . . . 82

4.7 Dilution factor for one of the $\mathrm{NH}_{3}$ target loads as a function of $W(\mathrm{GeV})$. $\quad 87$

$4.8 \quad$ Method Used in Estimating the Packing Fraction in SANE . . . . . . . . . 89

$4.9 \quad$ Radiation corrections mechanism _ . . . . . . . . . . . . . . . . . 90

$5.1 \quad$ Ratio of Background Particles to Electron High at Low $x \ldots \ldots$. . . . . 97

$5.2 \quad$ Charge Symmetric Background with SANE kinematics . . . . . . . . . . 97

5.3 Tracker Analysis, When Tracker is $\mathrm{In} /$ Out . . . . . . . . . . . . . . 101

5.4 Simulated Čerenkov Response with Double Track ADC Spectrum _ . . . . 102

5.5 Red-electron, blue-positron) starting direction of $40^{\circ}$ along the BETA axis . 103 
5.6 Vertical Deflection and Translation in the BigCal . . . . . . . . . . 103

5.7 Reconstructed $\pi^{0}$ Mass from Two Neutral Cluster Particles . . . . . . . . . 104

$5.8 \quad E(1)$ of Run $73001 \ldots \ldots \ldots \ldots \ldots$

$5.9 \quad E(2)$ of Run $73001 \ldots \ldots \ldots \ldots \ldots$

5.10 Comparing the MC (red plot) with Data . . . . . . . . . . . . . . 107

5.11 Average Asymmetry Versus Run Numbers for the Energy Cuts . . . . . . . 109

5.12 Total Average Asymmetry for All Cuts Versus Energy Cuts . . . . . . . . . 110

5.13 Average $\pi^{0}$ Physics Background Asymmetry (Blue Circles) . . . . . . . 111

5.14 Plots of $\pi^{+}$(Blue) and $\pi^{-}\left(\right.$Red) Asymmetries Versus $P_{T} \ldots \ldots \ldots$

5.15 Plots of $\pi^{+}$(Blue) and $\pi^{-}$(Red) Asymmetries Versus $P_{T} \ldots \ldots \ldots$

5.16 Plots of $\pi^{+}$(Blue) and $\pi^{-}$(Red) Asymmetries Versus $P_{T} \ldots \ldots \ldots$

5.17 Parallel $\pi^{0}$ Asymmetry Versus $P_{T}$ with a Constant Fit (Blue Line). . . . . . 120

5.18 Perpendicular $\pi^{0}$ Asymmetry Versus $P_{T} \ldots \ldots \ldots \ldots$

5.19 Fit to Perpendicular $\pi^{0}$ Asymmetry Data . . . . . . . . . . . . 122

5.20 Ratio of Background Particles to Electrons at SANE Kinematics . . . . . . 124

$6.1 \quad$ Plots of $A_{180}$ Versus $x$ at $4.7 \mathrm{GeV} \ldots \ldots \ldots \ldots$

$6.2 \quad$ Plots of $A_{80}$ Versus $x$ at $4.7 \mathrm{GeV} \ldots \ldots \ldots \ldots 131$

$6.3 \quad$ Plots of $A_{180}$ Versus $x$ at $5.9 \mathrm{GeV} \ldots \ldots \ldots \ldots$

$6.4 \quad$ Plots of $A_{80}$ Versus $x$ at $5.9 \mathrm{GeV} \ldots \ldots \ldots \ldots$

$6.5 \quad$ Plots of $A_{1}{ }^{p}$ Versus $W \ldots \ldots \ldots \ldots \ldots \ldots \ldots$

$6.6 \quad$ Plots of $A_{2}{ }^{p}$ Versus $W \ldots \ldots \ldots \ldots \ldots \ldots$

$6.7 \quad$ Plot of $A_{1}{ }^{p}$ Versus $x \ldots \ldots \ldots \ldots \ldots \ldots$ 
$6.8 \quad$ Results of $x^{2} g_{1}{ }^{p}$ Versus $x \ldots \ldots \ldots \ldots \ldots \ldots \ldots$

$6.9 \quad$ Results of $x^{2} g_{2}^{p}$ Versus $x \ldots \ldots \ldots \ldots \ldots \ldots \ldots$

$6.10 \quad$ Results of $g_{1}^{p}$ Versus $x \ldots \ldots \ldots \ldots$

$6.11 \quad$ Results of $x^{2} g_{2}{ }^{p}$ Versus $x \ldots \ldots \ldots \ldots \ldots$

A.1 Precession Difference Functions for Halls A and B . . . . . . . . . . 150

A.2 Precession Difference Functions for Halls B and C . . . . . . . . . 151

A.3 Precession Difference Functions for Halls A and C . . . . . . . . . . . 151 


\section{CHAPTER 1}

\section{INTRODUCTION}

The study of matter can be traced way back to $500 \mathrm{BC}[1]$ when the first speculations were recorded as to whether matter is continuous, or is composed of discrete particles by the Greek philosophers such as Anaxagoras (500-428 BC) and Empedocles (484-424 BC) as well as Leucippus (Circa 450 BC) and his pupil Democritus (460-370 BC) who argued that the universe consists of empty space and of indivisible particles, called atoms. The Greek word 'atomos' meaning indivisible, differing from each other in form, position and arrangement. The atomic hypothesis, however, was rejected by Aristotle (384-322 BC) who strongly supported the concept of the continuity of matter. The first experimental evidence that electric charge was not infinitely divisible, but existed in discrete units, was obtained by M. Faraday, who discovered the laws of electrolysis in 1833 [2]. While the first direct measurements of this smallest possible charge were initiated by J. J. Thomson and carried out by his student J. S. Townsend in 1879. In 1895, J. J. Thomas set out the hypothesis that cathode rays consisted of a stream of particles each of mass, $m$ and charge $-e$ [3]. Cathode ray is a beam of negatively charged electrons emitted from the cathode of a high-vacuum tube. All these were in attempt to address the question, "what is matter made of?" on the most fundamental level. In 1895, E. Rutherford was awarded a Research 
Fellowship to travel to England for postgraduate study at the Cavendish Laboratory, University of Cambridge to work under the inspiring leadership of J. J. Thomson [4]. By the early years of the 20th century, the atomic nature of matter had been well established. It was known that atoms contained electrons and that an electron was much lighter than even the lightest atom. The question now arises as to how the mass and positive charge are distributed within the atom. The answer was provided by Rutherford experiment in 1906 whereby Geiger and Marsden under Rutherford's supervision, scattered off $\alpha$-particles by metallic foils of various thickness [7]. Rutherford found that the positive charge, and most of the mass, was concentrated in a tiny core, or nucleus, at the center of the atom. The nucleus of the lightest atom (hydrogen) was given the name proton by Rutherford [5, 6]. In 1932, Chadwick discovered the neutron-an electrically neutral twin to the proton [8]. At this point the atom was known to be composed of just protons, neutrons, and electrons. Later, the supposed tiny protons and neutrons were discovered to possess internal structure. As the years went by (1930-1960) more elementary particles were discovered such as Yukawa's meson, Dirac's positron, and Pauli's neutrino. Many models were postulated to explain the mechanisms involved in the formation of these elementary particles. So far, the parton model is one of the most successful which has evolved into the quark model, in which the nucleons are comprised of quarks and gluons governed by the color force described by quantum chromodynamics [33].

Now, the structure of the nucleons can be probed and information about their constituent particles inferred from scattering of electrons from proton. In this document, one of such scattering experiments to probe the proton is discussed. The experiment was named 
SANE-Spin Asymmetry of the Nucleon Experiment with identification number E-07-003 and carried out at The Thomas Jefferson National Accelerator Facility (for short, JLAB).

\subsection{Lepton Scattering}

In considering scattering of a lepton from a nucleon, the cross section for such an interaction may be written in terms of a leptonic and a hadronic tensor. The fundamental interaction is the exchange of a virtual photon between the lepton and the nucleon. The leptonic tensor is known exactly through Quantum Electrodynamics (QED), but since the nucleon is not a fundamental particle, the hadronic tensor can be constrained but not known a priori. The typical approach is to write the hadronic tensor in terms of four structure functions that are functions of the kinematics of the interaction. The kinematic variables chosen are typically: $\nu$, and $-Q^{2}$ such that

$$
\begin{aligned}
\nu & =E-E^{\prime}, \\
Q^{2} & =4 E E^{\prime} \sin ^{2} \frac{\theta}{2} .
\end{aligned}
$$

Where $q^{2} \equiv-Q^{2}$ (with $Q^{2}>0$ ) is the 4 -momentum squared of the virtual photon and $\nu$ is the laboratory energy of the exchanged photon, $E$ and $E^{\prime}$ are the initial and final energies respectively, $\theta$ is the scattering angle of the photon. Two of the structure functions, $F_{1}\left(\nu, Q^{2}\right)$ and $F_{2}\left(\nu, Q^{2}\right)$ are known as the unpolarized structure functions and contribute to the cross section in all scattering events. The other two structure functions, $g_{1}\left(\nu, Q^{2}\right)$ and $g_{2}\left(\nu, Q^{2}\right)$ are known as the polarized structure functions and only contribute to the cross section if both the lepton and nucleon are polarized, hence cancel in the spin-averaged cross sections. 
The unpolarized structure functions were the first to be studied, in the 1970's and 1980's [10], and are now well known over a large kinematics range. The polarized structure functions on the other hand were studied next. They are most easily measured by determining the cross section asymmetry between two states that differ in either target or beam polarization direction. In the 1980's and 1990's, the polarized structure functions were measured in the so-called deep inelastic region, where $Q^{2}>1.0(\mathrm{GeV} / \mathrm{c})^{2}$ and $W>2 \mathrm{GeV}$, where $W$ is the mass of the hardronic state. In this region, they have been measured fairly accurately. The spin asymmetries $A_{1}\left(\nu, Q^{2}\right)$ and $A_{2}\left(\nu, Q^{2}\right)$ extend the deep inelastic scattering description of the nucleon spin structure to the region of the resonances. In this region, the nucleon spin structure can be described in terms of either the $g_{1}\left(\nu, Q^{2}\right)$ and $g_{2}\left(\nu, Q^{2}\right)$ spin structure functions (SSF) or the spin asymmetries, $A_{1}$ and $A_{2}$. The latter are constructed starting from the virtual photon absorption cross section $\sigma_{1 / 2}^{T}, \sigma_{3 / 2}^{T}$ and $\sigma_{1 / 2}^{T L}$ for photon helicities $+1,-1,0$, respectively.

$$
\begin{aligned}
& A_{1}=\frac{\sigma_{1 / 2}^{T}-\sigma_{3 / 2}^{T}}{\sigma_{1 / 2}^{T}+\sigma_{3 / 2}^{T}}=\frac{\nu M G_{1}\left(\nu, Q^{2}\right)-Q^{2} G_{2}\left(\nu, Q^{2}\right)}{W_{1}\left(\nu, Q^{2}\right)} \\
& A_{2}=\frac{\sigma^{T L}}{2 \sigma^{T}}=\sqrt{Q^{2}}\left(\frac{M G_{1}\left(\nu, Q^{2}\right)+\nu G_{2}\left(\nu, Q^{2}\right)}{W_{1}\left(\nu, Q^{2}\right)}\right)
\end{aligned}
$$

Where $2 \sigma^{T}=\sigma_{3 / 2}^{T}+\sigma_{1 / 2}^{T}, M$ is the nucleon mass and $W_{1}\left(\nu, Q^{2}\right)$ is the transverse unpolarized structure function. 
In the scaling limit of deep inelastic scattering (DIS), the structure function depends (up to logarithmic corrections) only on the scaling variable $x$, where

$$
\begin{aligned}
x & =\frac{Q^{2}}{2 M \nu}, \\
\lim _{Q^{2}, \nu \rightarrow \infty} M^{2} \nu G_{1}\left(\nu, Q^{2}\right) & =g_{1}(x), \\
\lim _{Q^{2}, \nu \rightarrow \infty} M \nu^{2} \nu G_{2}\left(\nu, Q^{2}\right) & =g_{2}(x), \\
\lim _{Q^{2}, \nu \rightarrow \infty} M W_{1}\left(\nu, Q^{2}\right) & =F_{1}(x) .
\end{aligned}
$$

When compared to scattering on longitudinally polarized nucleons, scattering longitudinally polarized electrons on transversely polarized nucleons provide access to $g_{1}$ and $g_{2}$ with different weighting. Thus allowing the spin-dependent structure functions to be separated experimentally. The measurement of $A_{2}$ from an experimental stand point is simpler than that of the absolute cross section difference for scattering of longitudinally polarized electrons on transversely polarized nucleons, which is required to access $g_{2}$ directly. Therefore, we aimed to measure the parallel $A_{180^{\circ}}$ and transverse (near perpendicular) $A_{80^{\circ}}$ asymmetries:

$$
\begin{aligned}
A_{80^{\circ}} & =\frac{-D^{\prime}}{W_{1}}\left\{\left[\left(E+E^{\prime} \cos \theta\right) \cos 80^{\circ}+E^{\prime} \sin \theta \cos \phi \sin 80^{\circ}\right] M G_{1}\right. \\
& \left.+\left(2 E E^{\prime} \sin \theta \cos \phi \sin 80^{\circ}-Q^{2} \cos 80^{\circ}\right) G_{2}\right\} \\
A_{180^{\circ}} & =\frac{D^{\prime}}{W_{1}}\left[\left(E+E^{\prime} \cos \theta\right) M G_{1}-Q^{2} G_{2}\right]
\end{aligned}
$$

which are related to the spin asymmetries $A_{1}$ and $A_{2}$ by:

$$
\begin{aligned}
& A_{1}=\frac{C}{D}\left(A_{\|}-d A_{\perp}\right) \\
& A_{2}=\frac{C}{D}\left(c^{\prime} A_{\|}+d^{\prime} A_{\perp}\right)
\end{aligned}
$$


Where $C, c^{\prime}, d, d^{\prime}$ and $D$ are functions of the kinematic variables only. However, $D$ has an additional mild dependence on the unpolarized structure function, $R\left(Q^{2}, W\right)=\sigma_{L} / \sigma_{T}$ such that

$$
D=\frac{1-\epsilon}{1+\epsilon R}
$$

$D$ contains the virtual photon polarization $\epsilon=1 /\left[1+2\left(1+\nu^{2} / Q^{2}\right) \tan ^{2} \theta / 2\right.$.

The spin structure functions are expressed in terms of these asymmetries and the structure functions as

$$
\begin{aligned}
g_{1} & =\frac{F_{1}}{1+\gamma^{2}}\left(A_{1}+\gamma A_{2}\right) \\
g_{2} & =\frac{F_{2}}{1+\gamma^{2}}\left(\frac{A_{2}}{\gamma}-A_{1}\right)
\end{aligned}
$$

Where $\gamma=4 x^{2} M^{2} / Q^{2}$

The Spin Asymmetry of the Nucleon Experiment (SANE) set out to extract $A_{1}$ and $A_{2}$ of the proton from measured asymmetries, extract $g_{1}$ and $g_{2}$ from $A_{1}$ and $A_{2}$, calculate the twist 3 matrix element $d_{2}$ of the proton which quantifies the quark-gluon interaction, probe as $x$ approached 1 at constant $Q^{2}$, and to test the quark models and pQCD prediction.

It is worth noting that the proton polarized structure function, $g_{2}^{p}$, has not been studied sufficiently. Actually, only six experiments so far have attempted to fully measurement $g_{2}^{p}$ namely, SLAC (E143, E155, E155x) [24, 23], CERN (NA-47) [25], and HERMES [13] being the most recent with DIS region covering data scattered over large $Q^{2}$ range, and Jefferson Lab (RSS) [20] with resonance region with data at $Q^{2} \sim 1.3 \mathrm{Ge} V^{2}$. More so, over the years there has been dearth of data on $A_{\perp}$ in the region of the $\left(Q^{2}, x\right)$ kinematic plane for $x>\sim 0.6[36,37]$. This region is entirely dominated by the nucleon resonances for $Q^{2} \leq 5 \mathrm{Ge} V^{2}$. Since the study of the spin structure function began, only parallel 


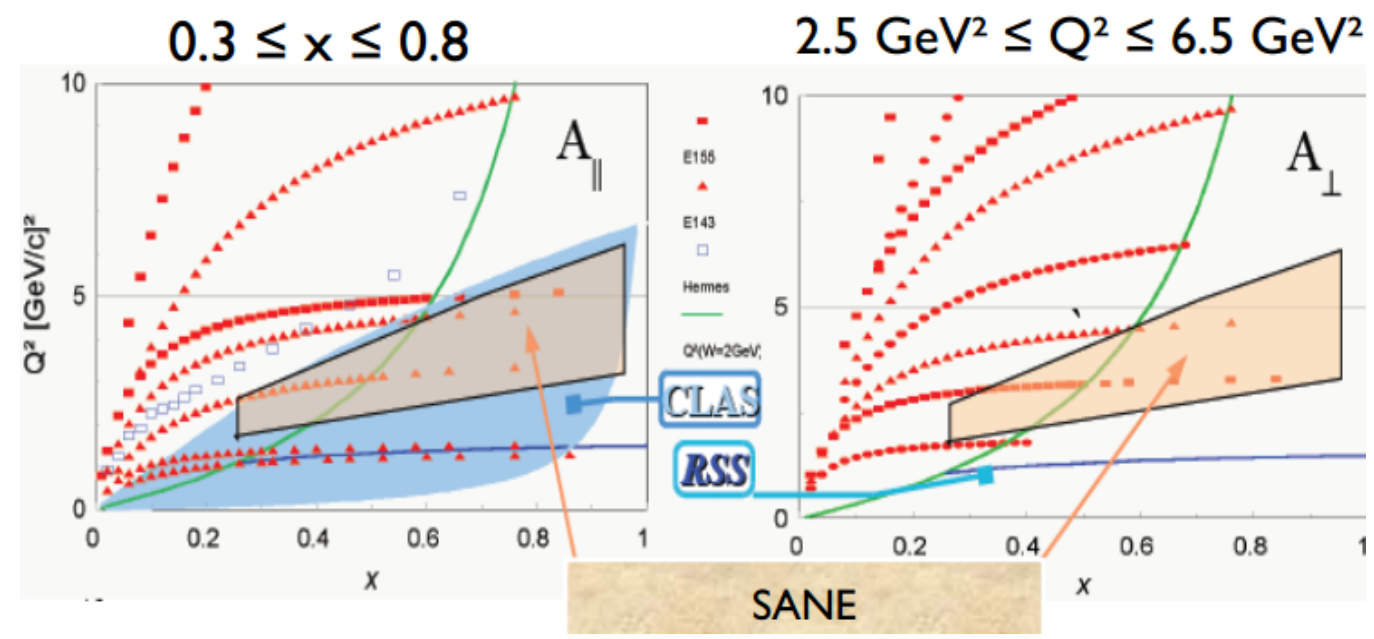

Figure 1.1

Plot of SANE Kinematics

asymmetry results for protons and deuteron have become available for most of the region. The only perpendicular asymmetry measurement on the protons and deuterons was done by the RSS collaboration in Jefferson Lab's Hall C [36] and it is limited to the resonances at $<Q^{2}>\sim 1.3 \mathrm{Ge} V^{2}$. Figure 1.1 depicts the situation for the two proton and deuteron targets where the absence of $A_{\perp}$ data at high $x$ is glaring. The case is somewhat better for the neutron where $g_{2}$ DIS measurement have been done in Jefferson's Lab Hall A.

Furthermore, this document takes a closer look into the pair-symmetric background coming from neutral particle decays as the beam traverses through the target. A full correction of the pair-symmetric background is important for a proper and reliable spin structure function study.

This thesis starts with an introduction in chapter one, followed by the theory section in chapter two. The detailed experimental setup and methods is presented in chapter three, 
while chapter four explains how the data were analyzed, chapter five explains the pair symmetric background. The results are presented in chapter six followed by the conclusion in chapter seven. 


\section{CHAPTER 2}

\section{THEORETICAL PERSPECTIVE}

\subsection{Investigating the Internal Structure of the Proton}

The building blocks of atomic nuclei are namely, protons and neutrons whose internal structure leads us to their fundamental properties. Having a full comprehension as to how the nucleons are built in terms of underlying quark and gluon degrees of freedom is of paramount importance to the field of Nuclear Physics.

Electron (as well as muon) scattering is a suitable tool to study the structure of the nucleon. Electrons can easily be accelerated in well defined monoenergetic beams and accurately detected using magnetic spectrometers and standard particle detection. Also, the

electromagnetic interaction is a "known" interaction with coupling strength of $\alpha_{e m}=\frac{1}{137}$, hence a good candidate for investigating the "picture" of the nucleon. Thus with such a well-defined interaction as the electromagnetic interaction, we can with confidence use it for a probe and a systematic calculation scheme for computing the results of experiments.

Two types of scattering mostly involved in the study of the nucleon are elastic and deep inelastic scattering. In elastic scattering, the final state of the nucleon is unchanged, but with a finite recoil. Here, the scattering cross section allows one to map out the charge and density distribution inside the nucleon. On the other hand, deep inelastic scattering (DIS) in which a quark in a nucleon gets knocked out by a virtual photon and the nucleon gets 
smashed into many fragments [10]. In this case, one gains access to the quark and gluon distribution in momentum space.

\subsection{Kinematics and Variable}

In order to calculate the cross section for elastic and deep inelastic scattering to the lowest order in weak or electromagnetic interactions, a lepton with momentum, $k$ scatters off a nucleon of mass $M$ with the exchange of a virtual photon or $Z^{0}$ or $W^{ \pm}$with momentum $q$ as shown in Figure 2.1.

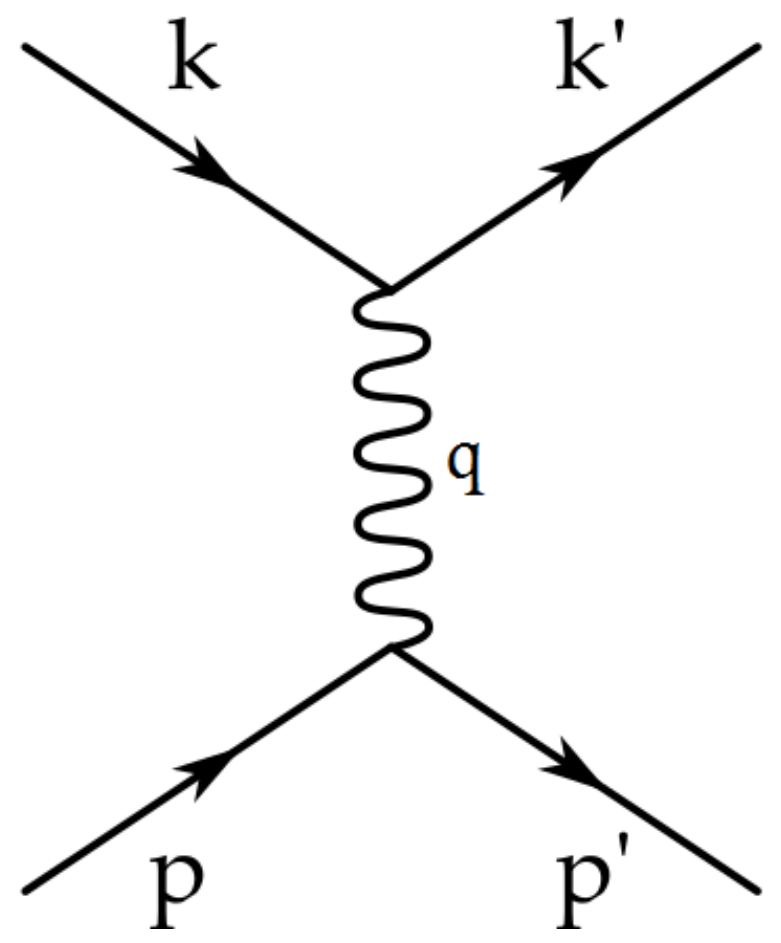

Figure 2.1

Elastic Scattering Showing the Four-momentum $q$ of the Virtual Photon 
We define the invariant quantity thus:

$$
\begin{aligned}
q^{2} & =\left(k-k^{\prime}\right)^{2}=-Q^{2}, Q^{2}>0, \\
s & =(p+k)^{2}, \\
W^{2} & =p_{x}^{2}, \\
\nu & =\frac{p \cdot q}{M}=\frac{1}{2 M}\left(W^{2}+Q^{2}-M^{2}\right) .
\end{aligned}
$$

\subsection{Electromagnetic Interaction}

Figure 2.1 shows an electron (or muon) scattering off a nucleon with an electron (or muon). Looking at the photon exchange, the neutral current cross section involves $\gamma$ and $Z^{0}$ exchange as well as the interference between the two. For $Q^{2}<10^{3} \mathrm{GeV}^{2}$ the electromagnetic interaction dominates.

\subsection{Elastic Scattering (Electron-Nucleon)}

Consider the elastic scattering picture in Figure 2.1 with the incident and outgoing electrons having four momenta $k=(E, \vec{k})$ and $k^{\prime}=\left(E^{\prime}, \overrightarrow{k^{\prime}}\right)$ respectively, the initial and final nucleon four momenta $P$ and $P^{\prime}$. With these kinematics variables, the four momentum of the virtual photon is given by:

$$
q=k-k^{\prime}=P^{\prime}-P \text {. }
$$

The virtual photon has invariant mass:

$$
q^{2}=-4 E E^{\prime} \sin ^{2} \frac{\theta}{2}
$$


where $\theta$ is the scattering angle. Equation (2.3) is always negative i.e., space-like in nature or by definition implying there is always a frame where by the energy transfer $q^{0}=\nu=0$ and $q^{2}=-\overrightarrow{q^{2}} \equiv Q^{2}$. This frame is known as the Breit frame.

Now looking at the elastic scattering condition of the lab frame, we have:

$$
\begin{aligned}
\left(P^{\prime}\right)^{2} & =(P+q)^{2}=M^{2} \\
& =M^{2}+2 P \cdot q+q^{2} \\
& =M^{2}+2 M \nu+q^{2} .
\end{aligned}
$$

Thus, $2 M \nu=Q^{2}$.

Figure 2.1 shows elastic scattering between a charged lepton and proton through a one photon exchange interaction. The energy loss is given by $\nu=E-E^{\prime}$ where $\nu$ is a delta function and it's given by $\nu=-q^{2} / 2 M$ corresponding to the scattered energy:

$$
E^{\prime}=\frac{E}{\left(1+\frac{2 E}{M} \sin ^{2} \frac{\theta}{2}\right)} .
$$

Again, $\theta$ is the scattering angle. In this interaction the physical observables characterizing compositeness are form factors, which enter the elastic scattering cross section. For example, in condense matter physics, it is also the form factors (or structure factors) that are probed in X-ray or electron scattering which roughly speaking, are the Fourier transformation of the charge density.

In Quantum Field Theory (QFT), the cross section for an interaction can be expressed as the product of the phase space factors, momentum, energy and the invariant matrix element. The invariant matrix element, $\mathcal{M}$ is the only part of the cross section which is dependent on the physics of the process being examined, thus much attention will be 
focused on calculating $\mathcal{M}$. Furthermore, in such a calculation, all quantities are averaged over the appropriate momenta and over the initial and final spin states. It should be noted that the averaging over spins state is the case for unpolarized scattering.

Now considering the scattering cross section in one photon exchange, we seek connection to the proton compositeness. If the electron photon vertex is $-i e \gamma^{\mu}$, the electron proton vertex is $(i e)\left\langle P^{\prime}\left|J^{\mu}\right| P\right\rangle$. The scattering matrix (S-matrix) element then reads:

$$
\begin{aligned}
S & =(2 \pi)^{4} \delta^{4}\left(k+P-P^{\prime}-k^{\prime}\right) \bar{u}\left(k^{\prime}\right)\left(-i e \gamma^{\mu}\right) u(k) \frac{-i}{q^{2}}\left\langle P^{\prime}\left|(i e) J^{\mu}\right| P\right\rangle \\
& =-i(2 \pi)^{4} \delta^{4}\left(k+P-P^{\prime}-k^{\prime}\right) \mathcal{M} .
\end{aligned}
$$

Where $\mathcal{M}$ is known as the invariant amplitude. The electromagnetic current is,

$$
J^{\mu}(\xi)=\sum_{i} e_{i} \vec{\psi}_{i}(\xi) \gamma^{\mu} \psi_{i}(\xi)
$$

where $\psi_{i}$ is the wave function, $i$ sums over all quark flavors: up, down, strange, charm, bottom, and top. The first three are light compared with the mass of the nucleon. The heavier quarks are ignored because they are heavy and play minor role. At this point we write the elastic scattering cross section $d \sigma$ in terms of the invariant amplitude as

$$
d \sigma=\frac{1}{2 k^{0} 2 P^{0}\left|\nu_{1}-\nu\right|}(2 \pi)^{4} \delta^{4}\left(k+P-P^{\prime}-k^{\prime}\right)\left|\mathcal{M}_{f_{i}}\right|^{2} \prod_{f} \frac{d^{3} P_{f}}{2 E_{f}(2 \pi)^{3}}
$$

where $\nu_{1}$ is the electron velocity, $\nu$ is the initial nucleon velocity, and $2 k^{0} 2 P^{0}\left|\nu_{1}-\nu\right|$ is invariant when boosted along the $z$-direction. However, in the laboratory frame, $k=E$, $P^{0}=M$, so we get:

$$
d \sigma=\frac{1}{2 M E}|\mathcal{M}|^{2} 2 \pi \delta\left((q+P)^{2}-M^{2}\right) \frac{d^{3} \overrightarrow{k^{\prime}}}{2 E^{\prime}(2 \pi)^{3}} .
$$


Integrating over $E^{\prime}=|\vec{k}|$, that is, when $m_{e}<<E^{\prime}$. We have

$$
\begin{aligned}
d \sigma & =\frac{E^{\prime}}{2 E M^{2}} \frac{1}{1+\frac{2 E}{M} \sin ^{2} \frac{\theta}{2}}|\mathcal{M}|^{2} \frac{d \Omega}{(2 \pi)^{2}} \\
& =\frac{E^{\prime}}{2 E M^{2}} \cdot f_{\text {rec }} \cdot|\mathcal{M}|^{2} \frac{d \Omega}{(2 \pi)^{2}}
\end{aligned}
$$

where $d \Omega$ is thge differential solid angle and $1 /\left(1+\frac{2 E}{M} \sin ^{2} \frac{\theta}{2}\right)$ is known as the recoil factor and reduces to unity if the particle is infinitely heavy. The invariant amplitude square is defined as

$$
|\mathcal{M}|^{2}=\frac{e^{4}}{Q^{4}} \ell^{\mu \nu}<P\left|J^{\nu}\right| P^{\prime}><P^{\prime}\left|J^{\mu}\right| P>
$$

where $\ell^{\mu \nu}$ is the lepton tensor

$$
\ell^{\mu \nu}=\bar{u}\left(k^{\prime}\right) \gamma^{\mu} u(k) \bar{u}(k) \gamma^{\nu} u\left(k^{\prime}\right)
$$

For unpolarized scattering, we average over the initial polarization and sum over the final polarization state to obtain

$$
\ell^{\mu \nu}=2\left(k^{\prime \mu} k^{\nu}+k^{\prime \nu} k^{\mu}-g^{\mu \nu} k^{\prime} \cdot k\right)
$$

where $g^{\mu \nu}$ is the matrix tensor. The hadron tensor is thus given by

$$
W^{\mu \nu}=\left\langle P\left|J^{\nu}\right| P^{\prime}\right\rangle\left\langle P^{\prime}\left|J^{\mu}\right| P\right\rangle
$$

which depends on the current matrix element. The matrix element of the current between the nucleon states defines two form factors,

$$
\left\langle P^{\prime}\left|J^{\mu}(0)\right| P\right\rangle=\bar{U}\left(P^{\prime}\right)\left[F_{1}\left(Q^{2}\right) \gamma^{\mu}+F_{2}\left(Q^{2}\right) \frac{i \sigma^{\mu \nu} q_{\nu}}{2 M}\right] U(P)
$$

where $\sigma^{\mu \nu}$ is the cross section matrix tensor and $F_{1}\left(Q^{2}\right)$ is known as the Dirac form factor and $F_{2}\left(Q^{2}\right)$ is the Pauli form factor. They are parameterizations of the unknown correction 
to the electron vertex. An experiment can thus be carried out to measure the $Q^{2}$ dependence of the cross section in order to extract $F_{1}$ and $F_{2}$ from the data. Once data on $F_{1}$ and $F_{2}$ have been collected, the electron properties can be calculated. Furthermore, the anomalous part of the magnetic moment for the proton is $\kappa_{p}=\mu_{p}-1$, in nuclear magneton-units, $e \hbar /(2 M c)$, with value $\kappa_{p}=1.7928 . M$ is the nucleon mass and $\mu_{p}$ is the magnetic moment of the proton. It follows that in the static limit, $Q^{2}=0, F_{1 p}(0)=1, F_{2 p}(0)=\kappa_{p}$. It should be pointed out that the structure functions, which the SANE data allow access to, are the inelastic analogs of the form factors and will be discussed later.

Also, the electric and magnetic form factors are related in the so called Sachs electric and magnetic form factors as:

$$
\begin{aligned}
& G_{E}\left(Q^{2}\right)=F_{1}\left(Q^{2}\right)-\tau F_{2}\left(Q^{2}\right) \\
& G_{M}\left(Q^{2}\right)=F_{1}\left(Q^{2}\right)+F_{2}\left(Q^{2}\right) .
\end{aligned}
$$

Where $\tau=Q^{2} / 4 M^{2}$.

Thus the hadron tensor now takes the form

$$
\begin{aligned}
W^{\mu \nu}= & 2\left(P^{\prime \mu} P^{\nu}+P^{\prime \nu} P^{\mu}-g^{\mu \nu}\left(P P^{\prime}-M^{2}\right)\right) G_{M}^{2} \\
& -2 F_{2} G_{M}\left(P+P^{\prime}\right)^{\mu}\left(P+P^{\prime}\right)^{\nu} \\
& +F_{2}^{2} \frac{M^{2}+P \cdot P^{\prime}}{2 M^{2}}\left(P+P^{\prime}\right)^{\mu}\left(P+P^{\prime}\right)^{\nu} \\
= & \left(-q^{\mu} q^{\nu}+g^{\mu \nu} q^{2}\right) G_{M}^{2}+\left(P+P^{\prime}\right)^{\mu}\left(P+P^{\prime}\right)^{\nu} \frac{G_{E}^{2}+\tau G_{M}^{2}}{1+\tau} \\
= & g^{\mu \nu} q^{2} G^{2}{ }_{M}+4 P^{\mu} P^{\nu} \frac{G_{E}^{2}+\tau G_{M}^{2}}{1+\tau}+\ldots
\end{aligned}
$$

here, the ellipses indicate terms involving factors of $q^{\mu}$ which do not contribute to the cross section because of the current conservation. 
Considering that the lepton and the hadron tensors are symmetric and conserved in the lab frame i.e., $q^{\mu} \ell_{\mu \nu}=q^{\mu} W_{\mu \nu}$, the elastic scattering cross section becomes:

$$
\frac{d \sigma}{d \Omega}=\sigma_{M o t t}\left[\frac{G_{E}^{2}\left(Q^{2}\right)+\tau G_{M}^{2}\left(Q^{2}\right)}{1+\tau}+2 \tau G_{M}^{2} \tan ^{2} \frac{\theta}{2}\right],
$$

where $\sigma_{M o t t}$ is the Mott scattering cross section which represent the scattering of the electron from a point-like scalar proton. In terms of the recoil factor, $f_{r e c}$ :

$$
\sigma_{M o t t}=\frac{Z^{2} \alpha^{2} \cos ^{2} \frac{\theta}{2}}{4 E^{2} \sin ^{4} \frac{\theta}{2}} f_{r e c}
$$

were $Z$ is the atomic number. If the proton were structureless, then $G_{E}=G_{M}=1$ and the cross section would be:

$$
\frac{d \sigma}{d \Omega}=\sigma_{M o t t}\left[1+2 \tau G_{M}^{2} \tan ^{2} \frac{\theta}{2}\right]
$$

Any observed deviation from this is a clear indication of nucleon substructure.

The cross section can be written as

$$
\frac{d \sigma}{d \Omega}=\frac{\sigma_{M o t t}}{1+\tau}\left[G_{E}^{2}\left(Q^{2}\right)+\frac{\tau}{\epsilon} G_{M}^{2}\left(Q^{2}\right)\right]
$$

where $\epsilon^{-1}$ being the virtual proton longitudinal polarization is given by

$$
\epsilon^{-1}=(1+\tau) 2 \tan \frac{\theta}{2}
$$

With the need to extract the elastic and magnetic form factors separately from the elastic scattering, we endeavor to measure two cross sections at fixed $Q^{2}$ by varying the scattering angle $\theta$ and hence $\epsilon$ since they are correlated by equation (2.22). If we plot the quantity in the square bracket of equation (2.21) versus $\epsilon^{-1}$, the intercept and slope provide separately, the electric and magnetic form factors. This method of separation is the so called Rosenbluth separation method [12]. 


\subsection{Deep Inelastic Scattering and The Parton Model}

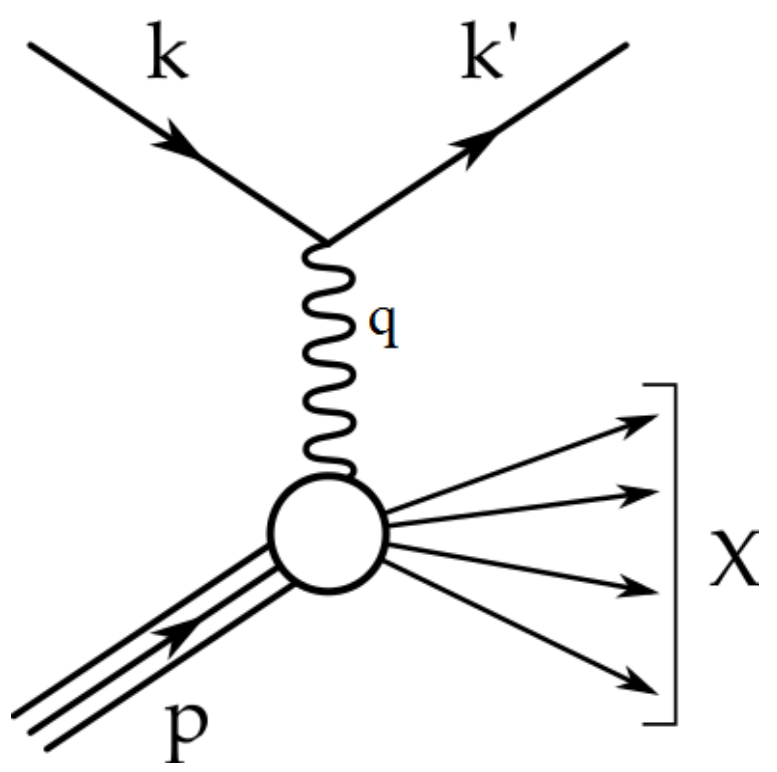

Figure 2.2

Deep Inelastic Scattering

Deep inelastic scattering (DIS) is the process used to probe the inside of hadrons (particularly baryons, such as protons and neutrons) using electrons, muons and neutrinos [46]. An example to consider is the proton in the nucleus of a hydrogen atom. If one strikes it with some momentum transfer $q$, by measuring its final momentum $\overrightarrow{k^{\prime}}$, one can figure out the initial momentum $\vec{k}$. If the energy transfer (momentum transfer) is kept low enough, that energy would be absorbed as a change in momentum; the final state is strickly determined by the kinematics of the electron, in which case the electron and hadron are elastically scattered off of one another as described in the preceding section. On the other hand, if the energy transfer is increased, it is possible to excite nucleon resonances, in- 
duce pion production or a range of other processes. This kind of scattering is referred to as inelastic as shown in Figure 2.2. To explore the existence of quarks inside the proton, good knowledge of their distributions in momentum space is highly needed. To do this, we scatter highly virtual photons off the quarks in the proton and measure the distribution. Considering electron scattering off a proton producing a final state $|X\rangle$ as shown on Figure 2.2. Using the one photon exchange approximation, the S-matrix is:

$$
S=(2 \pi)^{4} \delta^{4}\left(k+P-P^{\prime}-k^{\prime}\right) \bar{u}\left(k^{\prime}\right)\left(-i e \gamma^{\mu}\right) u(k) \times \frac{-i}{q^{2}}\left\langle X\left|(i e) J^{\mu}\right| P\right\rangle,
$$

where $X$ is any hadronic final state. The corresponding inclusive cross section is

$$
\frac{d \sigma}{d \Omega d E}=\frac{\alpha^{4}}{Q^{4}} \frac{E^{\prime}}{E} \ell_{\mu \nu} W^{\mu \nu}
$$

Inclusive in this case means that the final state of the target is not detected, and consequently the measurement "includes" all the different reactions of the electron with the target, $e p \longrightarrow e^{\prime} X$ (where $X$ is any final state) and the hadronic tensor is given by

$$
W_{\mu \nu}=\frac{1}{4 \pi} \sum_{X}\left\langle P\left|J_{\mu}\right| X\right\rangle\left\langle X\left|J_{\nu}\right| P\right\rangle(2 \pi)^{4} \delta^{4}\left(P+q-P_{x}\right) .
$$

The $W$ tensor depends only on the initial nucleon momentum $P$ and the photon momentum $q$. Making use of Lorentz symmetry, parity and time reversal invariance and current conservation, the $W$ tensor can be written in terms of two invariant tensors, $W_{1}$ and $W_{2}$ as shown:

$$
W_{\mu \nu}=W_{1}\left(-g^{\mu \nu}+\frac{q^{\mu} q^{\nu}}{q^{2}}\right)+\frac{W_{2}}{M^{2}}\left(P^{\mu}-q^{\mu} \frac{P \cdot q}{q^{2}}\right)\left(P^{\nu}-q^{\nu} \frac{P \cdot q}{q^{2}}\right)
$$

Where $W_{1}$ and $W_{2}$ are functions of $Q^{2}$ and $\nu\left(Q^{2}\right.$ and $\nu$ being Lorentz scalars). When $W_{1}$ and $W_{2}$ are plotted as a function of $x=Q^{2} / 2 M \nu$, they are nearly independent of $Q^{2}$. This 
is known as Bjorken scaling. Whereas, if $x$ is fixed, $Q^{2}$ tends to $\infty$ and called the Bjorken limit.

The Parton model introduced by Feynman was used to explain the fact that the nucleon is made of non-interacting partons (quarks). More so, in deep inelastic scattering, the photons scatters off these free partons. Partons can be any particles with no internal structure and must be interacting in order to prevent the nucleon from falling apart. To calculate the hadronic tensor, we sum over scattering on partons

$$
W^{\mu \nu}=\int \frac{d x_{F}}{x_{F}} f\left(x_{F}\right) \omega^{\mu \nu},
$$

where $x_{F} P$ is the longitudinal momentum carried by a parton, $f\left(x_{F}\right)$ is the parton density and $\omega^{\mu \nu}$ is the hadron tensor for a single quark. Taking into consideration the contribution of the anti quark

$$
W^{\mu \nu}=-\frac{1}{4 \pi} \operatorname{Im} \int d x \operatorname{Tr}\left[\gamma^{\mu} \frac{x p+q}{(x p+q)^{2}+i \epsilon} \gamma^{\nu} p f_{1}(x)\right]+\text { crossing } .
$$

Upon performing the trace, we arrive at

$$
W^{\mu \nu}=\frac{1}{2 \nu}\left[f_{1}\left(x_{B}\right)-f_{1}\left(-x_{B}\right)\right]\left(2 x_{B} p^{\mu} p^{\nu}+p^{\mu} q^{\nu}+p^{\nu} q^{\mu}-g^{\mu \nu} \nu\right) .
$$

Where $-f_{1}\left(-x_{B}\right)$ is the contribution of $\bar{f}\left(x_{B}\right)$, the anti parton. Now, comparing this with the definition of the structure functions

$$
\begin{aligned}
& W_{1}=\frac{1}{2} \sum_{i} e_{i}^{2}\left[f_{1}^{i}\left(x_{B}\right)+\bar{f}_{1}^{i}\left(x_{B}\right)\right], \\
& W_{2}=\frac{M}{\nu} x_{B} \sum_{i} e_{i}^{2}\left[f_{1}^{i}\left(x_{B}\right)+\bar{f}_{1}^{i}\left(x_{B}\right)\right] .
\end{aligned}
$$


Here, we have restored the summation over quark flavor and included the weight of quark charges. Thus, we define the scaling functions

$$
\begin{aligned}
& F_{1}\left(x_{B}\right)=W_{1}=\frac{1}{2} \sum_{i} e_{i}^{2}\left[f_{1}^{i}\left(x_{B}\right)+\bar{f}_{1}^{i}\left(x_{B}\right)\right] \\
& F_{2}\left(x_{B}\right)=\frac{\nu}{M} W_{2}=x_{B} \sum_{i} e_{i}^{2}\left[f_{1}^{i}\left(x_{B}\right)+\bar{f}_{1}^{i}\left(x_{B}\right)\right],
\end{aligned}
$$

where we see clearly the relation $F_{2}\left(x_{B}\right)=2 x_{B} F_{1}\left(x_{B}\right)$, the well known Callan-Gross relation. It should be clear that no assumption are made about the quark interactions before scattering in the derivation given above.

To conclude on this sub section on probing the nucleon structure through DIS, we have considered the proton to be comprised of two up valence quarks and one down quark $(u u d)$ with electric charge $2 / 3$ and $-1 / 3$ of the proton. There are infinite number of quarks; this can be seen because, the integration $\int q(x) d x$ does not seem to converge due to the fact that there are infinite number of quarks and antiquark pairs in the proton.

It is also worth pointing out that the gluons have been found to play very important role in the nucleon structure. By constructing the integral $\int x q(x) d x$, the fraction of the nucleon momentum carried by quarks can be calculated. Experimental data indicate that this is only about $50 \%$ thereabout. Thus the missing momentum must be carried by the gluons. Hence, the charge-neutral gluons is of great importance in the determination of the nucleon structure.

\subsection{Physics of Parton Distribution}

In this section, we consider a model of the nucleon to describe the physical meaning of the structure function, $F_{2}(x)$. This model is call the Quark Parton Model and considers 
the nucleon to be composed of point-like free particles (partons) with momentum parallel to the proton's. Looking into the parton distributions, we start by writing out the structure functions assuming only up and down quarks are present. This is because, quantitatively, the up and down quarks dominate the structure functions. For simplicity, we define the up and down momentum fraction distributions as:

$$
\begin{aligned}
& u(x) \equiv f_{u}(x) \\
& d(x) \equiv f_{d}(x)
\end{aligned}
$$

And likewise for the antiquarks. The structure functions can thus be written:

$$
F_{2}^{p}(x)=x\left\{\frac{4}{9}\left[u^{p}(x)+\bar{u}^{p}(x)\right]+\frac{1}{9}\left[d^{p}(x)+\bar{d}^{p}(x)\right]\right\}
$$

Under isospin flip, $u \longleftrightarrow d$ and $n \longleftrightarrow p$; this implies:

$$
F_{2}^{n}(x)=x\left\{\frac{4}{9}\left[d^{p}(x)+\bar{d}^{p}(x)\right]+\frac{1}{9}\left[u^{p}(x)+\bar{u}^{p}(x)\right]\right\}
$$

Letting $u(x) \equiv u^{p}(x)$ and $d(x) \equiv d^{p}(x)$

$$
\begin{aligned}
& F_{2}^{p}=x\left[\frac{4}{9}(u+\bar{u})+\frac{1}{9}(d+\bar{d})\right] \\
& F_{2}^{n}=x\left[\frac{4}{9}(d+\bar{d})+\frac{1}{9}(u+\bar{u})\right]
\end{aligned}
$$

From these expressions, it can be deduced that the inequality:

$$
\frac{1}{4} \leq \frac{F_{2}^{n}}{F_{2}^{p}} \leq 4
$$

holds. On the other hand it can be shown that the ratio of the neutron and proton unpolarized structure function $F_{2}(x)$ in the quark model is:

$$
\frac{F_{2}^{n}}{F_{2}^{p}}=\frac{1+4 d^{p}(x) / u^{p}(x)}{4+d^{p}(x) / u^{p}(x)}
$$




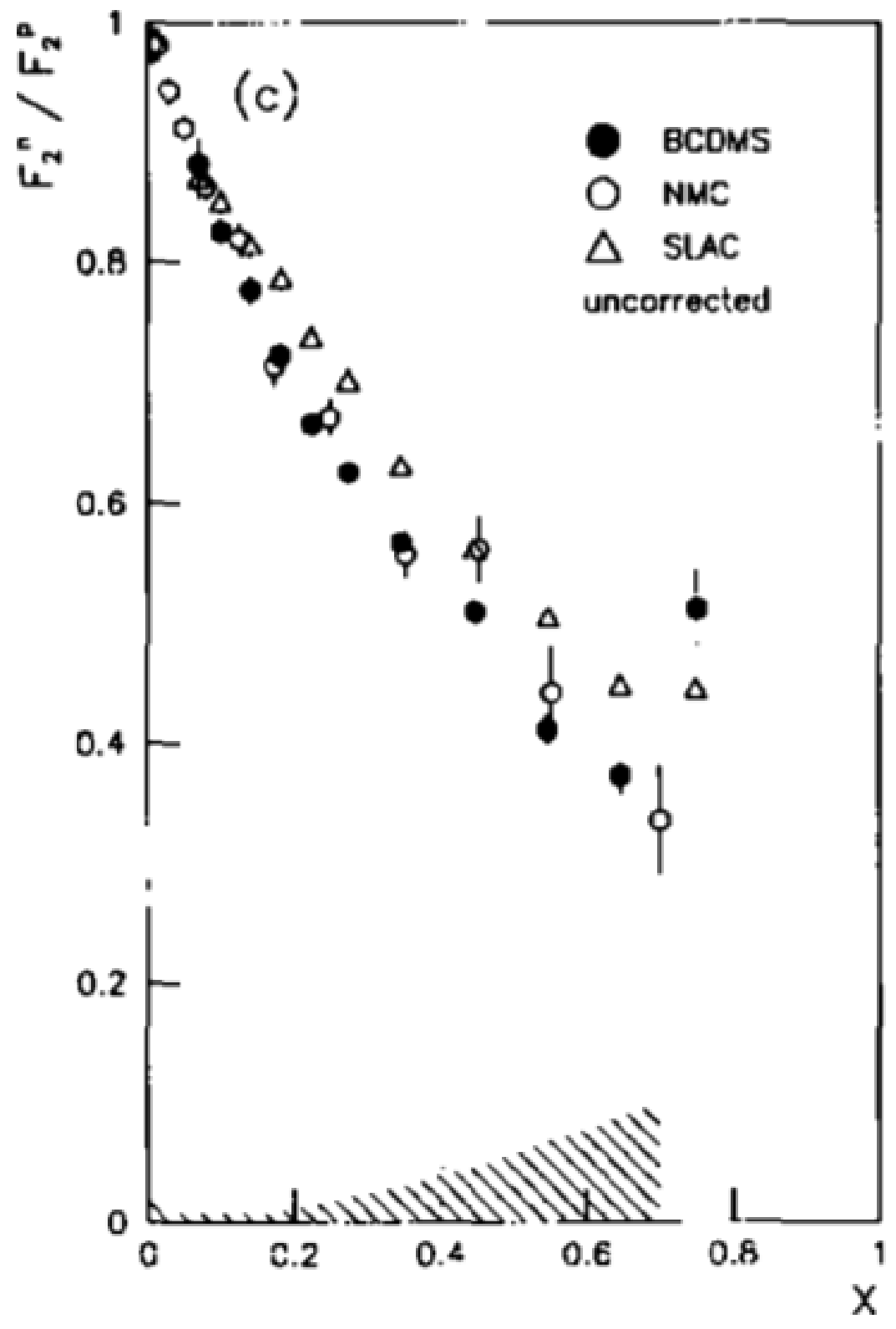

Figure 2.3

Plot Showing $F_{2}^{n} / F_{2}^{p}$ Ratio 
Figure 2.3 [14] shows the plotted data of the ratio of equation (2.37). This ratio approaches the limit of $1 / 4$ as $x \longrightarrow 1$, which is a clear indication of a negligible down quark contribution to the composition of the proton. On the other hand, if $A_{1}(x)$ approaches 1 at high $x$, then the scattering process is dominated by high momentum up quarks with angular momentum aligned with that of the proton. In the region of low $x ; x \ll 1$, the 'sea' (quarks) of $q \bar{q}$ pairs dominates the structure function and one observes:

$$
\frac{F_{2}^{n}}{F_{2}^{p}} \longrightarrow 1
$$

At higher $x \longrightarrow 1$, the valence quarks dominate and we find:

$$
\frac{F_{2}^{n}}{F_{2}^{p}} \longrightarrow \frac{1}{4}
$$

$u(x)>d(x)$ in the proton since there are 2 valence up quarks verses only one valence down quark. The parton model postulates that the nucleon is constituted of point-like free particles with momentum parallel to the proton's. In the scaling limit, the structure functions, $F_{1}\left(Q^{2}, x\right)$ and $F_{2}\left(Q^{2}, x\right)$ can be written as their corresponding asymptotic value in this limit. Beside direct experimental observation of the quark structure by measuring $F_{1}\left(Q^{2}, x\right)$ and $F_{2}\left(Q^{2}, x\right)$, it is possible to obtain evidence of the existence of a sea of gluon in the nucleon. Furthermore, the gluon carry a significant fraction of the momentum of the nucleon which affects a "momentum" sum rule that indicates the fraction of the momentum carried by the quarks. For scattering from an isoscalar nucleus such as deuterium, we define:

$$
\begin{aligned}
F_{2}^{N}(x) & \equiv \frac{1}{2}\left(F_{2}^{p}+F_{2}^{n}\right) \\
& =\frac{5}{18} x[u(x)+\bar{u}(x)+d(x)+\bar{d}(x)]
\end{aligned}
$$


Therefore,

$$
\frac{18}{5} \int_{0}^{1} F_{2}^{N}(x) d x=\int_{0}^{1} x \sum_{i} f_{i}(x) d x
$$

This shows that one can measure the sum of the momentum fractions of all the quarks (including antiquarks) via the integral of equation (2.41). If there were no other significant constituents then the above integral should be unity. Notwithstanding, experimental observation shows that

$$
\frac{18}{5} \int_{0}^{1} F_{2}^{N}(x) d x=0.50 \pm 0.05
$$

The picture of Figure 2.4 shows experimental data for the structure function $F_{2}(x)$ for the proton at various values of $x$ versus the squared momentum transfer $Q^{2}$. The data are plotted as a function of $Q^{2}$ in bins of fixed $x$ [34].

\subsection{Quark Spin Structure of the Nucleon}

When the spins of the quarks are probed in polarized deep inelastic scattering in which the lepton and target proton are both polarized along the scattering axis, the polarized electron exchanges a polarized virtual photon with the target. Due to helicity conservation, the virtual photon inherits some of the incident lepton helicity, resulting in a virtual photon with some net helicity. Thanks to helicity conservation a $(+)$ helicity quark can only absorb a $(+)$ helicity photon, likewise a $(-)$ helicity quark can only absorb a $(-)$ helicity photon. By studying the difference under reversal of the proton spin (photon helicity) we obtain the probability that the struck quark has the same helicity as the incident lepton for a fixed spin orientation of the proton. The cross section difference:

$$
\Delta \sigma=\sigma_{++}-\sigma_{+-}
$$




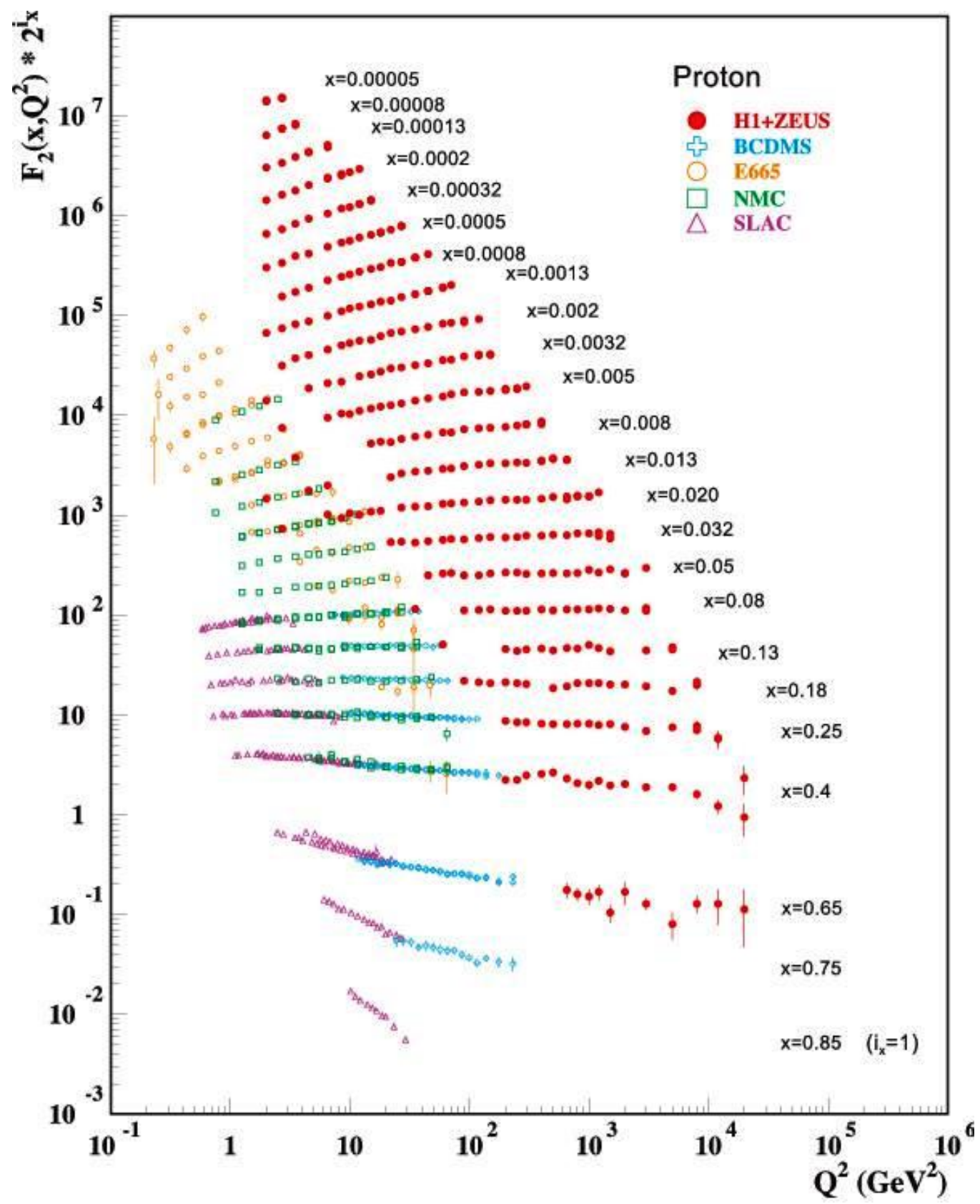

Figure 2.4

Experimental data of the proton structure function $F_{2}^{p}(x)$ at various values of $x$ 
is proportional to the combination of polarized momentum fraction distribution (spin structure function):

$$
\Delta \propto \frac{1}{2} \sum_{i} e^{2}\left[q_{i}^{+}(x)-q_{i}^{-}(x)\right] \equiv g_{1}(x)
$$

Where $q_{i}^{+(-)}$is defined to be the momentum fraction distribution for quark spin parallel (antiparallel) to the nucleon spin. In the case where the nucleon target is polarized with a spin vector $S$, the hadronic tensor, $W^{\mu \nu}$ will contain terms depending on $S$ as follows

$$
W^{[\mu \nu]}=-i \epsilon^{\mu \nu \alpha \beta} q_{\alpha}\left[G_{1}\left(\nu, Q^{2}\right) S_{\beta} / M^{2}+G_{2}\left(\nu, Q^{2}\right)\left(S_{\beta} \nu M-P_{\beta} S \cdot q\right) / M^{4}\right]
$$

We have two scaling functions in the Bjorken limit:

$$
\begin{aligned}
& g_{1}\left(x, Q^{2}\right)=\left(\frac{\nu}{M}\right) G_{1}\left(\nu, Q^{2}\right) \longrightarrow g_{1}(x), \\
& g_{2}\left(x, Q^{2}\right)=\left(\frac{\nu}{M}\right)^{2} G_{1}\left(\nu, Q^{2}\right) \longrightarrow g_{2}(x),
\end{aligned}
$$

these two functions are non-vanishing. The spin structure function $g_{1}$ (and $g_{2}$ ) is extracted from the measured asymmetries of the scattering cross section as the beam or target spin is reversed. It should be noted that $g_{1}$ (and $g_{2}$ ) can also be extracted from differences of polarized cross section [27]. These asymmetries are measured with longitudinally polarized beams and longitudinally $\left(A_{\|}\right)$and transversely $\left(A_{\perp}\right)$ polarized targets

$$
\begin{aligned}
A_{\|} & =\frac{\sigma^{\downarrow \Uparrow}-\sigma^{\uparrow \Uparrow}}{\sigma^{\downarrow \Uparrow}+\sigma^{\uparrow \Uparrow}}, \\
A_{\perp} & =\frac{\sigma^{\downarrow \Rightarrow}-\sigma^{\uparrow \Rightarrow}}{\sigma^{\downarrow \Rightarrow}+\sigma^{\uparrow \Rightarrow}},
\end{aligned}
$$

where " $\Uparrow$ " represents the target spin polarization and " $"$ " represents the electron beam helicity, $\sigma^{\uparrow \Uparrow}$ and $\sigma^{\downarrow \Uparrow}$ represent the polarized cross sections when the longitudinal spins of the target and the incoming electron beam are aligned and anti-aligned, respectively. Similarly, $\sigma^{\downarrow \Rightarrow}$ and $\sigma^{\uparrow \Rightarrow}$ represent the cross sections for the two electron helicities states 
on transversely polarized target. Furthermore, $A_{\|}$and $A_{\perp}$ are combined to get $A_{1}$ and $A_{2}$ by using equation (1.5). Lastly, $g_{1}$ and $g_{2}$ are obtained from $A_{1}, A_{2}$ and $F_{1}$ as in equation (1.7).

Therefore, by measuring these asymmetries the experimental values for the spin dependent structure functions $g_{1}(x)$ and $g_{2}(x)$ can be determined. Figures ?? and ?? show world data for $g_{1}^{p}$ and $g_{2}$. However, the cross-section differences are very small and measurements of individual cross sections are limited by experimental systematic uncertainties. In order to avoid the time dependent systematic effects, the polarization of the target/beam was flipped frequently. While it was hard to flip the target polarization, but the CEBAF beam polarization is flipped at $60 \mathrm{~Hz}$, so the systematics were limited to the beam charge asymmetry and the live time correction.

\subsection{Moments and Twist}

For the $n$th moment of $F_{1}$ and $F_{2}$, the moments or $x$-weighted integrals of the structure functions can be defined as:

$$
\begin{aligned}
& M_{1}^{(n)}\left(Q^{2}\right)=\int_{0}^{1} d x x^{n-1} F_{1}\left(x, Q^{2}\right) \\
& M_{2}^{(n)}\left(Q^{2}\right)=\int_{0}^{1} d x x^{n-2} F_{2}\left(x, Q^{2}\right)
\end{aligned}
$$

These are also known as the Cornwall-Norton moments [65]. We note that for $n=1, F_{1}$ is described to have an effective count of quark charges, while for $n=2, F_{2}$ depicts the momentum sum rule. 


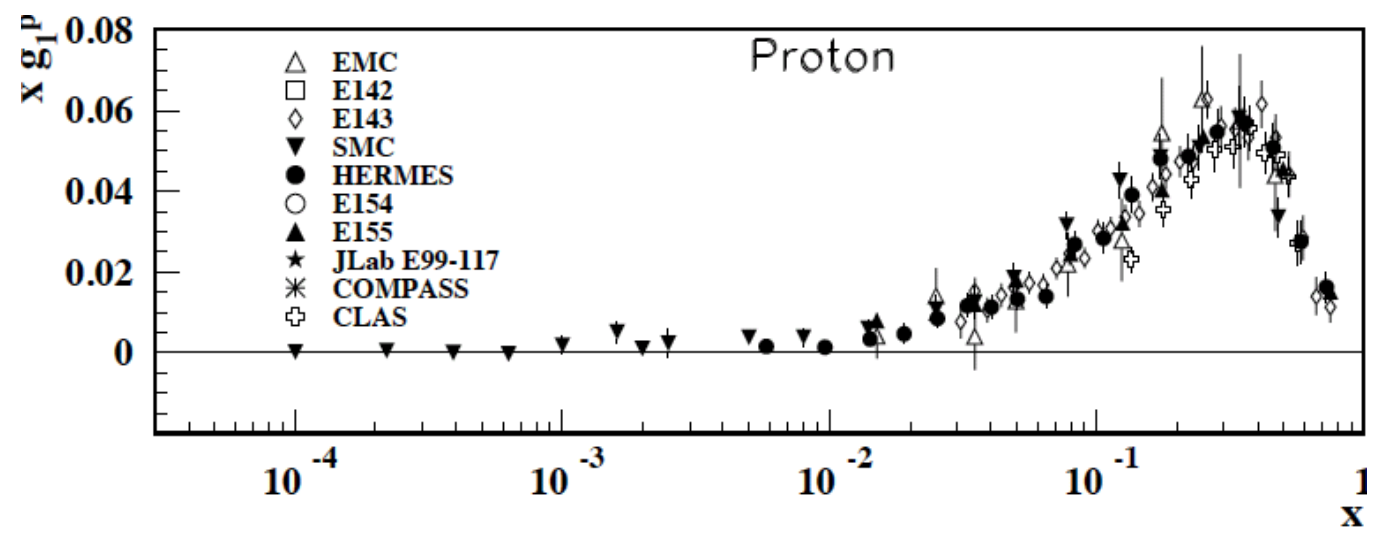

Figure 2.5

World data of $g_{1}^{p}$

Furthermore, the spin structure function moments are defined as:

$$
\begin{aligned}
& \Gamma_{1}^{(n)}\left(Q^{2}\right)=\int_{0}^{1} d x x^{n-1} g_{1}\left(x, Q^{2}\right) \\
& \Gamma_{2}^{(n)}\left(Q^{2}\right)=\int_{0}^{1} d x x^{n-2} g_{2}\left(x, Q^{2}\right)
\end{aligned}
$$

With the difference of the first moments of the proton and neutron longitudinal spin structure functions, $g_{1}^{p}$ and $g_{1}^{n}$ is the fundamental Bjorken sum rule, namely;

$$
\Gamma_{1}^{p}\left(Q^{2}\right)-\Gamma_{1}^{n}\left(Q^{2}\right)=\int_{0}^{1}\left(g_{1}^{p}\left(x, Q^{2}\right)-g_{1}^{n}\left(x, Q^{2}\right)\right) d x=\frac{1}{6} \frac{g_{A}}{g_{V}} C_{N}\left(\alpha_{s}\right) .
$$

Where $g_{A} / g_{V}$ correspond to the ratio of the axial vector and vector coupling constants, which can be determined by measurements of angular correlations in neutron decay. $g_{T}$, the transverse spin structure function given by $g_{T}=g_{1}+g_{2}$ comes from the corresponding twist-3 piece of the spin structure function $g_{2} . g_{2}$ consists of a twist-2 part $g_{2}^{W W}$ and a mixed twist-2/twist-3 part $\overline{g_{2}}$. However, the twist-2 part of $g_{2}$ can be extracted once $g_{1}$ is measured [35]. Figure 2.5 shows world data for $g_{1}^{p}$ [45].

$$
g_{2}\left(x, Q^{2}\right)=g_{2}^{W W}\left(x, Q^{2}\right)+\overline{g_{2}}\left(x, Q^{2}\right) .
$$


From the inclusive measurements in DIS, it has been established that the quarks carry only about $25 \%$ of the nucleon spin $[38,39,40]$ and that there are small but non-negligible quark-gluon interactions in the region $0.2 \leq x \leq 0.4$.

$$
\begin{aligned}
g_{2}^{W W}\left(x, Q^{2}\right) & =-g_{1}\left(x, Q^{2}\right)+\int_{x}^{1} g_{1}\left(y, Q^{2}\right) \frac{d y}{y} \\
\overline{g_{2}}\left(x, Q^{2}\right) & =-\int_{x}^{1} \frac{\partial}{\partial y}\left[\frac{m}{M} h_{T}\left(y, Q^{2}\right) y+\xi\left(y, Q^{2}\right)\right] \frac{d y}{y}
\end{aligned}
$$

where $m$ and $M$ are the quark and nucleon masses, and $h_{T}\left(x, Q^{2}\right)$ is the chiral-odd quark transverse spin distribution. $\xi$ represents a leading twist-3 contribution from processes involving quark-gluon interactions [37]. In addition to $g_{2}^{W W}, g_{2}$ consists of another twist-2 contribution from the transversity, $h_{T}$, even though in DIS this contribution is suppressed by the ratio $m / M[41,42,43]$. Because of the suppression of $h_{T}$, the third moment of the mixed twist $\overline{g_{2}}\left(x, Q^{2}\right)$ can be related by the operator product expansion (OPE) to the reduced twist-3 quark matrix element $d_{2}$. The OPE is a connection between quark matrix elements of the nucleon and the moments of the spin structure functions,

$$
\int_{0}^{1} x^{2} \overline{g_{2}}\left(x, Q^{2}\right) d x=\frac{1}{3} d_{2}\left(Q^{2}\right)
$$

$d_{2}$ can be calculated in lattice QCD [76]. With $h_{T}$ being a leading twist quantity and hence comparable in magnitude to $g_{1}$, this implies that even if the $m / M$ ratio were to be of the order of $\sim 1 \%, h_{T}$ could represent a significant contribution to $\overline{g_{2}}$, since the pure twist-3 part, $\xi$ may be considerably smaller than $g_{1}$.

In addition to the motivations of the SANE as discussed in section 1.1, only a limited amount of $d_{2}$ data exist from SLAC and RSS at Jefferson Lab. Hence SANE aimed to determine $\overline{g_{2}}$ with precision in the critical region $0.3 \leq x \leq 0.8$ at fixed $Q^{2}$, and compute 
$d_{2}$ by combining SANE and world data. Figure 2.6 and Figure 2.7 show the expected results of $g_{1}$ and $g_{2}$ respectively [16]. Figure 2.8 shows the expected results of SANE for $\bar{d}_{2}$, plotted on the projected pQCD evolution of $d_{2}$, normalized to SLACs C-N result at 5 $\mathrm{Ge} V^{2}$. RSS result and the lattice QCD calculation are shown.

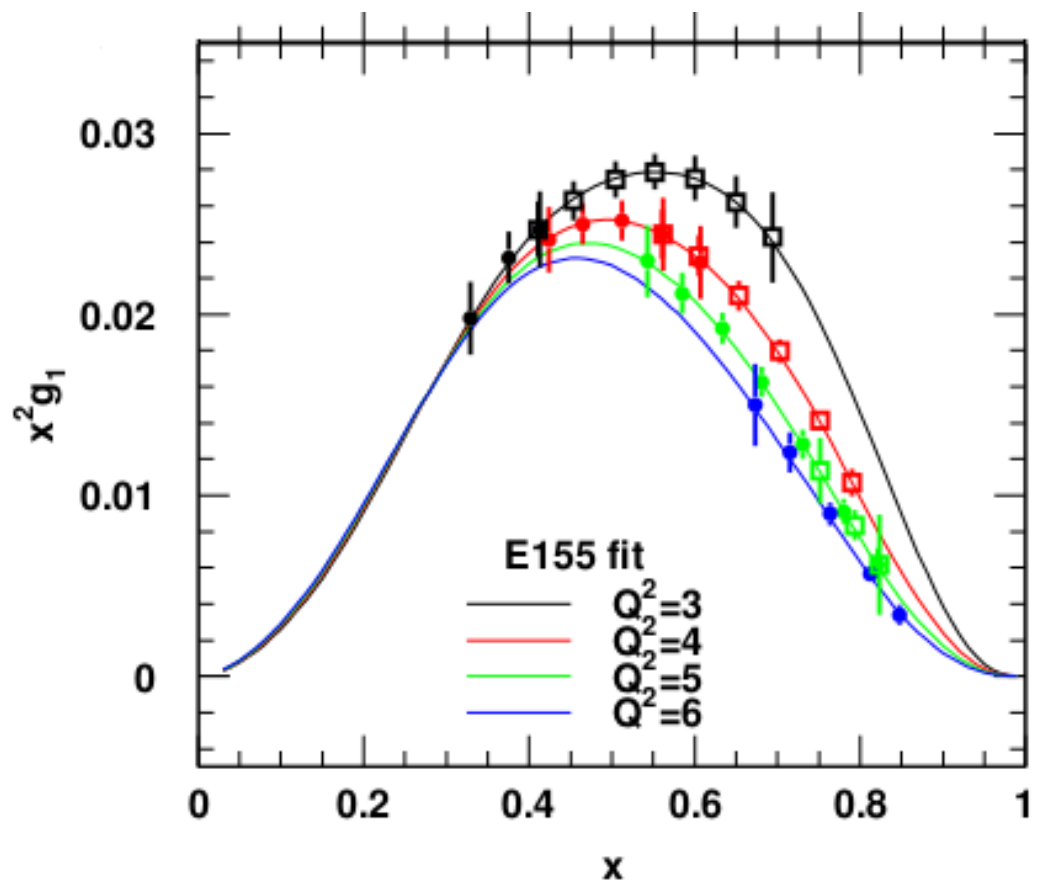

Figure 2.6

Data of $g_{1}$ plotted versus $x$

More relevant to $g_{2}$ is the fact that it gives access to the polarizabilities of the color field [44] taking into account the twist-4 matrix element $f_{2}$. The magnetic and electric polarizabilities are:

$$
\begin{aligned}
& \chi_{B}=\frac{\left(4 d_{2}+f_{2}\right)}{3}, \\
& \chi_{E}=\frac{\left(2 d_{2}-f_{2}\right)}{2}
\end{aligned}
$$




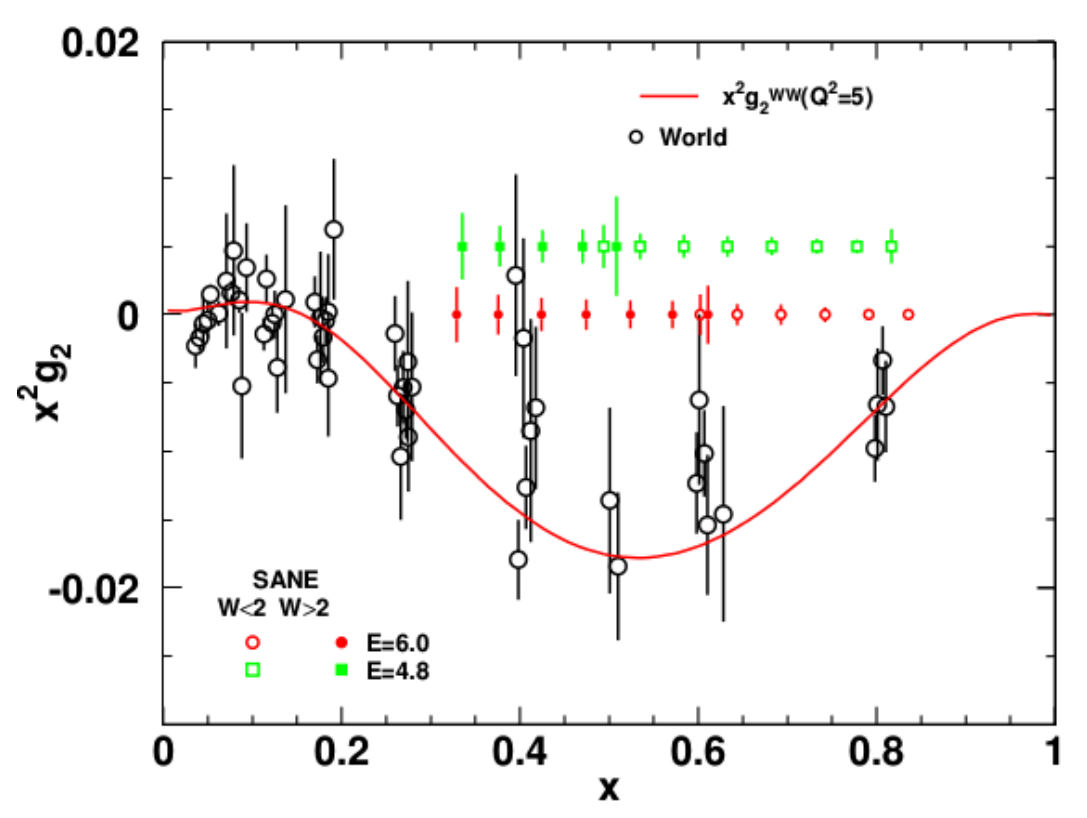

Figure 2.7

Data of $g_{2}$ plotted versus $x$, with $g_{2}^{W W}$

respectively.

Knowledge of these properties of the color fields is an important step in understanding QCD. The twist $-4 f_{2}$ matrix element represents, the quark-quark interactions, and reflects the higher twist corrections to the individual proton and neutron moments of $g_{1}$ and in consequence to the Bjorken sum rule [64, 44].

$$
\int_{0}^{1} g_{1}\left(x, Q^{2}\right) d x=\frac{1}{2} a_{0}+\frac{M^{2}}{9 Q^{2}}\left(a_{2}+4 d_{2}+4 f_{2}\right)+O\left(\frac{M^{4}}{Q^{4}}\right)
$$

where $a_{0}, a_{2}, d_{2}, f_{2}$ correspond to the twist- 2 , quark mass, twist- 3 and twist- 4 terms respectively. More so, these matrix elements are related to the higher moments of the spin structure function and have a strong dependence on the high $x$ contributions. 


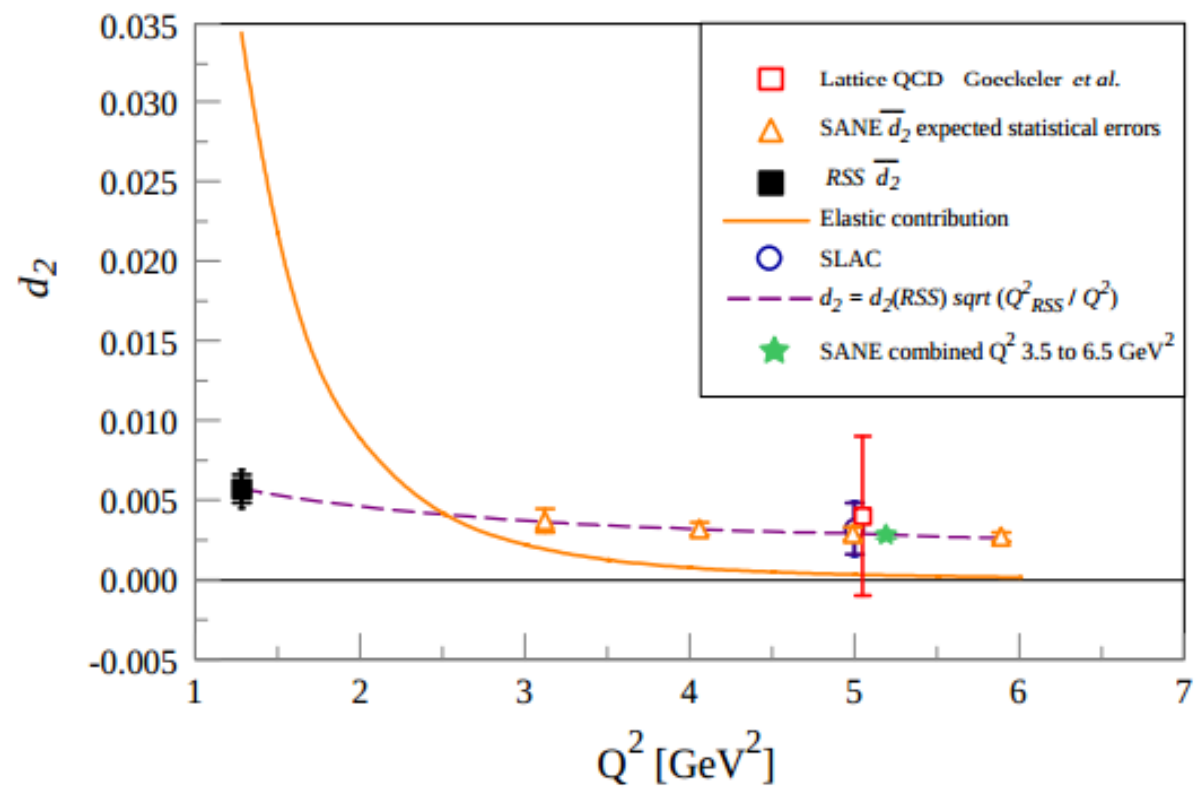

Figure 2.8

Expected SANE results for $\bar{d}_{2}$ 


\section{CHAPTER 3}

\section{EXPERIMENTAL SETUP}

SANE, was conducted from January to March of 2009 at the Thomas Jefferson National Accelerator Facility under experiment number E03-007. The experiment was aimed to extract $A_{1}$ and $A_{2}$ from measured asymmetries, and extract $g_{1}$ and $g_{2}$ from $A_{1}$ and $A_{2}$; with $g_{1}$ and $g_{2}$ one can calculate the Twist 3 matrix element $d_{2}$ given by

$$
d_{2}=\int_{0}^{1} x^{2}\left(2 g_{1}+3 g_{2}\right) d x
$$

(which quantifies the quark-gluon interaction). In addition, the experiment probes the approach of $A_{1}$ to $x=1$ at constant $Q^{2}$ which tests the quark models and pQCD prediction. In this chapter, an insight into the description of the experiment is presented starting with an overview of the experimental methods presented in section 3.1. This is followed by the polarized electron beam in section 3.2 and description of the various detector components in section 3.3. Lastly, section 3.4 present the triggers and data acquisition system.

\subsection{Experimental Apparatus}

The SANE experiment was carried out in Hall C of Jefferson Lab (JLab). Jefferson Lab was ideal for SANE for the following reasons:

- The high polarization continuous electron beam. 


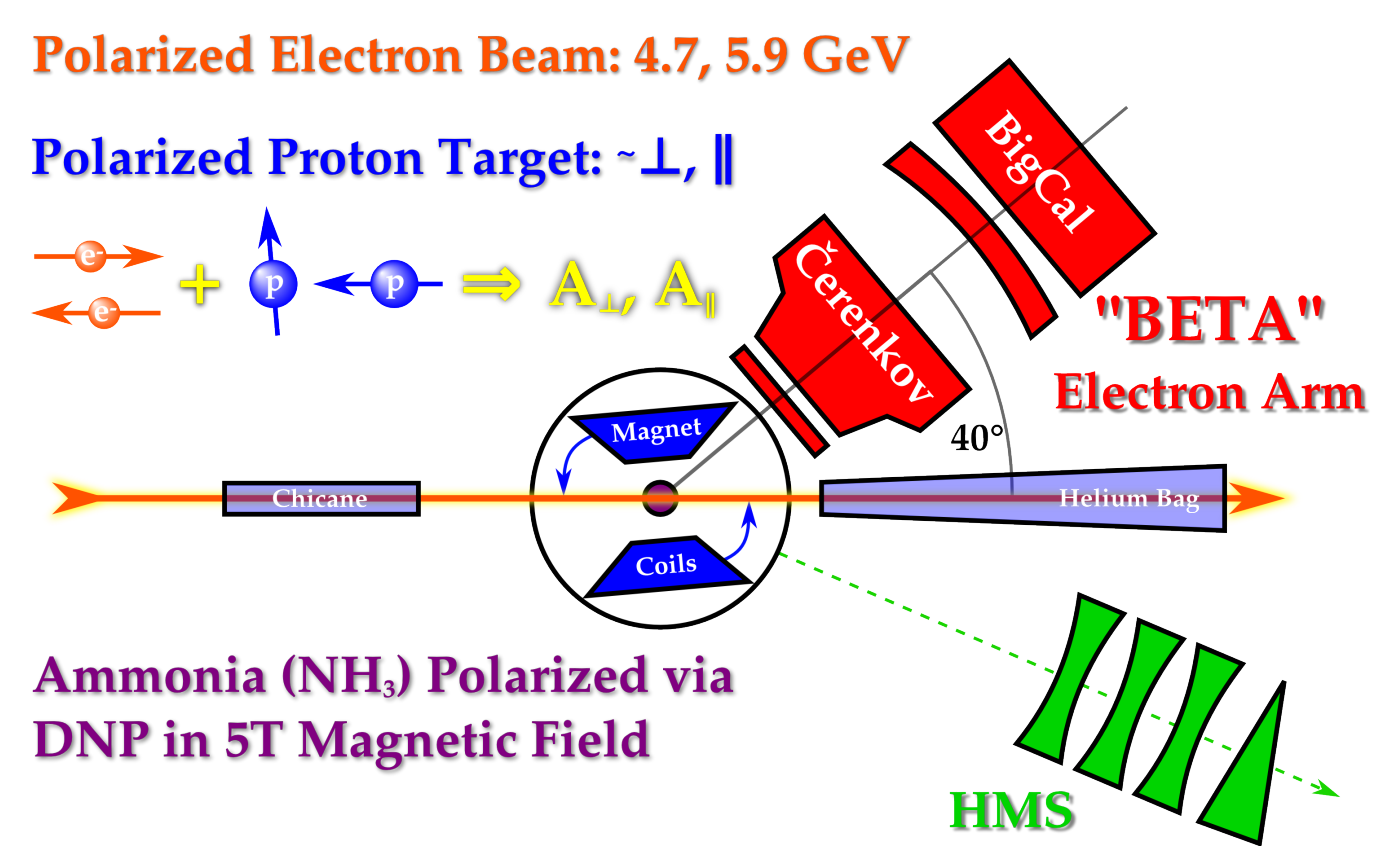

Figure 3.1

Experimental Layout Showing the Various Components Involved

- The large solid angle of the Hall C Cerenkov, calorimeter, tracking hodoscopes detector system, BETA ("Big Electron Telescope Array"), which makes possible high statistics measurements at $Q^{2} \sim 5 \mathrm{Ge} V^{2}$ in reasonable amount of run time [76].

- The open geometry of the UVa solid polarized target, that allows for flexible relative orientations of the beam helicity and the target spins, coupled with the high proton polarizations [16].

Figure 3.1 shows the experimental layout of SANE showing the various components involved.

\subsection{The Accelerator}

The primary mission of Jefferson Lab is to conduct basic research of the atom's nucleus using its unique particle accelerator. The Continuous Beam Accelerator Facility (CEBAF) beam produced at Jefferson Lab is based on superconducting radiofrequency (SRF) tech- 
nology. It produces a stream of charged electrons use to probe the nucleus of the atom. It is the world's most advanced particle accelerator for investigating the quark structure of the atom's nucleus.

The accelerator provides electron beams for experiments and uses a sophisticated computer system to control hundreds of thousands of hardware components which includes a complex cryogenic, microwave, vacuum and magnet systems that comprise the accelerator. Jefferson Lab operates two superconducting radiofrequency accelerators: the Continuous Electron Beam Accelerator Facility (CEBAF) and the Free-Electron Laser (FEL).

The CEBAF accelerator is a unique accelerator used to conduct investigations in the field of nuclear physics. It provides high-current, medium-energy electron beams concurrently to three experimental halls for the study of quarks and gluons, protons and neutrons and the nucleus of the atom. The Jefferson Lab Free-Electron Laser, though powered by a smaller SRF accelerator, holds power records in the production of infrared, ultraviolet and terahertz beams. CEBAF consists of two, anti-parallel linear accelerators, each capable of approximately $600 \mathrm{MeV}$ of acceleration. These accelerators are joined together through a series connection via nine recirculating arcs, five at the north end and four at the south end, to form a "race-track" such that after five passes through the linacs it's possible to provide a maximum beam energy of approximately $6 \mathrm{GeV}$. After extraction, the accelerator can deliver polarized, continuous wave beam at currents up to $200 \mu \mathrm{A}$ to be divided among the three experimental halls, A, B, and C (See Figure 3.2). 


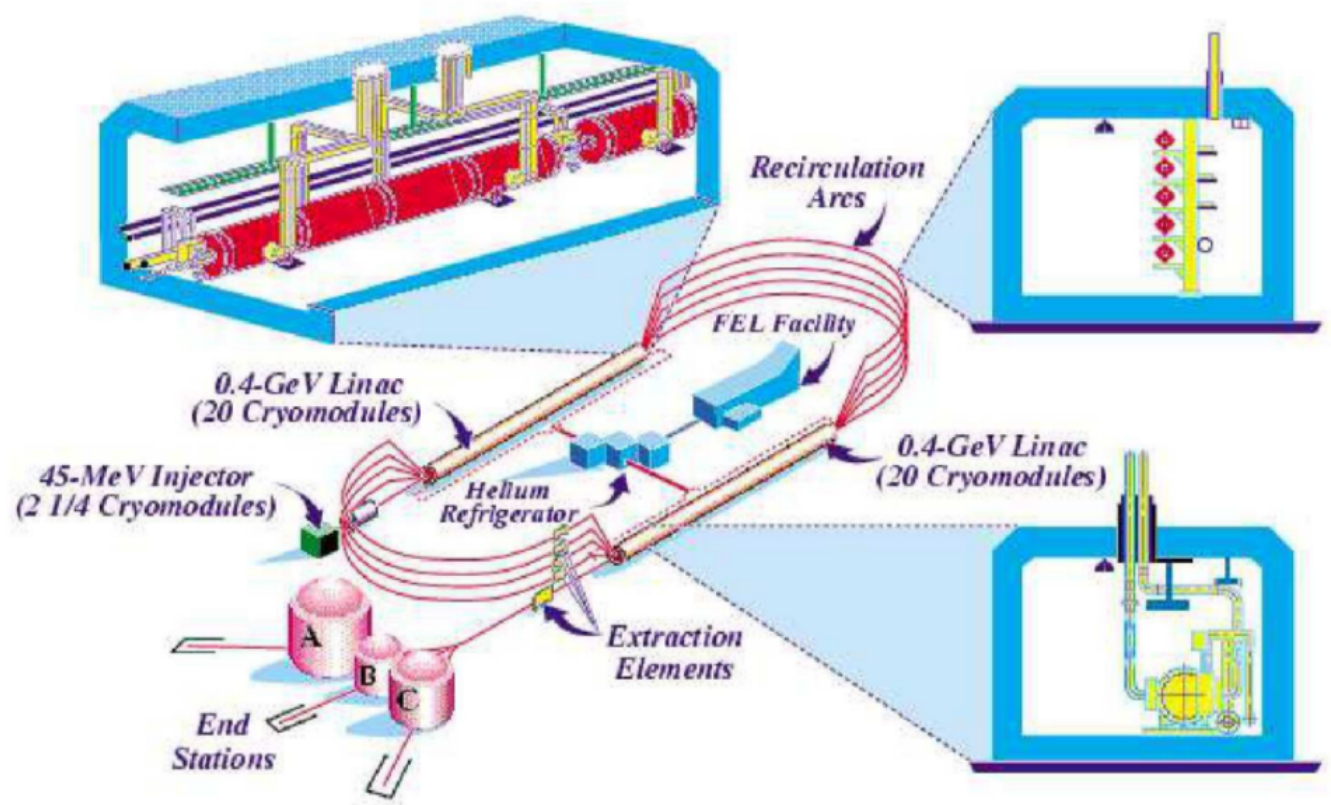

Figure 3.2

An Annotated Diagram of the CEBAF Accelerator

\subsubsection{The Polarized Beam Source}

The source is a polarized electron beam. The polarized electrons were excited by circular polarization from a semiconductor photocathode and accelerated for the SANE experiment to energies of $4.7 \mathrm{GeV}$ and $5.9 \mathrm{GeV}$ by the superconducting radio-frequency resonant cavities of the CEBAF accelerator. When the electrons were accelerated, they were delivered to Hall $\mathrm{C}$ experimental hall where they collided with a $\mathrm{NH}_{3}$ target.

\subsubsection{The Polarized Electron Production}

The starting point of the production and acceleration of the electron beam is at the electron source where electrons are excited from a strained GaAs (Gallium arsenide) crystal using circularly polarized laser light. The polarization of the electrons take place by 
optical pumping the $P_{3 / 2}$ valence-band level and the $S_{1 / 2}$ conduction-band level in GaAs. Moreover, a monolayer of $\mathrm{Cs}_{2} \mathrm{O}$ (Caesium oxide) on the GaAs causes the surface with negative electron affinity to shift the vacuum level below the conduction band, resulting in the release of excited photoelectrons across the semiconductor bandgap into the vacuum. All this is possible thanks to the fact that GaAs has a direct bandgap. In GaAs, the four spin substates of $P_{1 / 2}$ and $P_{3 / 2}$ level are degenerate, so light of the band-gap energy will induce transitions of both $P_{1 / 2}$ and $P_{3 / 2}$. With respect to the Clebsch-Gordan angular momentum coupling coefficient between the initial and excited states, there is $50 \%$ theoretical limit of polarized electrons. Notwithstanding, by lifting the degeneracy of the $P_{3 / 2}$, the polarization can be made higher than this limit.

Higher polarizations are reached by lifting the degeneracy through mechanically staining the GaAs. One way to implement the strain is by growing the GaAs cathode on a substrate of GaAsP, which has a different lattice constant. With so called "superlattice" doped on every other layer via phosphorus, Jefferson Lab is able to strain GaAs cathodes to deliver $85 \%$ polarized electron beam with a QE (Quantum Efficiency) of about 1\%.

Three gain switched diode lasers, one for each experimental hall are used to produce electrons from the cathode. Each laser is pulsed at a frequency of $499 \mathrm{MHz}$, and the three lasers are phased shifted relative to each other by $120^{\circ}$. Each laser pulse produces a single bunch of electrons, and the combined train of electron bunches has a frequency of 1497 $\mathrm{MHz}$, which equals the fundamental resonant frequency of the RF accelerating cavities in the linacs. 


\subsubsection{Acceleration and Delivery of Beam}

A $100 \mathrm{KV}$ electron gun accelerates the electrons from the polarized source into the injector. Furthermore, the injector provides as much as $67 \mathrm{MeV}$ of additional acceleration as it sends the electrons into the north linear accelerator. Each linear accelerator and the injector consist of 21, 20 and 4 cryomodules respectively; these cryomodules themselves contain 8 superconducting RF cavities as well a support cryogenics and power. Each cavity provides a nominal acceleration of about $28 \mathrm{MeV}$, giving each linac a nominal acceleration of $570 \mathrm{MeV}$. At 5 passes through the race track this provides $5.9 \mathrm{GeV}$. The accelerating cavities are made from Niobium cooled to $2 \mathrm{~K}$, with liquid helium and each is powered by an RF klystron at $1497 \mathrm{KHz}$. Electrons move on the crest of the RF wave in the superconducting cavities, gaining energy while their speed remains close to the speed of light. Because the electrons are already relativistic after leaving the injector, they are tuned to stay in phase with the RF field in the cavities. They will remain so even after several linac passes. In this way the cavities carry as many as 5 sets of electron beams from each successive pass simultaneously. As soon as the beam reaches the end of a linac, a series of dipole magnets splits the beam according to their energy, routing each to a recirculating arc. These arcs steer the beam back around to the other linac, with each successive arc using a larger field integral to carry beam of higher momentum around the turn in the race-track. As the beam switches yard, it is extracted from the racetrack which uses RF separator magnets at $499 \mathrm{MHz}$ to separately extract the 3 beams after any number of passes to send to each of the three experimental halls. 


\subsubsection{Beam Current Measurement}

Beam Current Monitors (BCM1 and BCM2) are used to measured the current of the beam entering the hall. BCM1 and BCM2 are cylindrical cavities designed to resonate in the transverse magnetic mode $T M_{010}$ at the same frequency as the accelerator RF. They are used to monitor the beam current in real time. The $T M_{010}$ mode is desirable mainly because the output power is relatively insensitive to the beam position inside the cavity when the beam is close to the cavity's longitudinal axis. Furthermore, this mode is excited as the beam passes through these cavities and the antennae placed inside the cavities are used to convert the RF power of the excited resonance, which is proportional to the square of the beam current. The final BCM1 and BCM2 are sent to a scaler which is read out every two seconds by the data acquisition running the Experimental Physics Industrial Control System (EPICS). It should be worth noting that the gain of the BCM cavities is quite sensitive to temperature due to its shape and size and the fact that the cavity can expand and contract in response to charges in temperature.

The drifting of the $\mathrm{BCM}$ gain over time necessitate periodic recalibration to ensure accuracy of the real time measurement of the current. The Unser monitor, which can be described as a parametric current transformer with an extremely stable gain was used as an absolute standard against which to calibrate the BCM cavities and calculate the correct gain of the BCM. However, the Unser monitor is not suitable to monitor the current in real time like the BCM1 and $\mathrm{BCM} 2$ due to the fact that the Unser monitor suffer from an unstable zero offset which can drift significantly over a short time scale. 
To calibrate the $\mathrm{BCM}$ using the Unser monitor with no beam current in the cavities, alternating runs are taken and then likewise with beam on of various currents so as to establish the zero offset and the gain respectively.

\subsubsection{Beam Energy Measurement}

To measure the beam energy as it enters Hall $\mathrm{C}$, the arc dipole magnets are used as a spectrometer. At the entrance, exit and middle of the arc, pairs of superharps precisely measure the position and direction or angle of the beam before and after the arc. In addition, a pair of superharps at the midpoint of the arc provides a third measurement of the trajectory and determines its curvature. With these measurements of the curvature of the beam over its $34.3^{\circ}$ deflection by the dipoles, the beam energy can be determined using the precise knowledge of the field integral of the arc dipole as a function of current:

$$
E \simeq P=\frac{e}{\theta} \int \vec{B} \cdot \overrightarrow{d l}
$$

where $e=$ electric charge, $\theta=$ arc bend angle, $\vec{B}=$ magnetic field integral over the path of the beam.

Table 3.1 shows the arc energy measurement; the average reading of the beam energy measurement, average per run for each beam energy and target field.

\subsubsection{Beam Position Measurement}

The beam position monitor (BPM) is used to measure the beam position at every point within the beam line. Each BPM is made up of a resonant cavity possessing a fundamental frequency that matches that of the accelerator and the Hall $\mathrm{C}$ beam. As a measure to 
Table 3.1

Beam Energies per Run for the various SANE run periods

\begin{tabular}{cccc}
\hline Normal E $(\mathrm{GeV})$ & Target field angle & Avg E(MeV) & Standard Deviation \\
\hline 4.7 & $180^{\circ}$ & 4736.7 & 0.9 \\
4.7 & $80^{\circ}$ & 4728.5 & 0.8 \\
4.7 & $80^{\circ}$ & 4729.1 & 0.5 \\
5.9 & $180^{\circ}$ & 5895.0 & 1.9 \\
5.9 & $80^{\circ}$ & 5892.1 & 4.9 \\
\hline
\end{tabular}

minimize synchrotron radiation damage, the cavity (holding four antennae) is rotated by $45^{\circ}$ relative to the vertical and horizontal axes.

The BPM also ensures that the beam trajectory makes it way exactly to the center of the SANE target passing through the $2.5 \mathrm{~cm}$ diameter target cup. Furthermore, information about the beam energy is also made available by the BPM as described in the previous subsection. Figure 3.3 shows the BPMs $(\mathrm{mm})$ versus all the experimental runs numbers.

\subsubsection{Beam Polarization Measurement}

The beam polarization was measured using a Møller polarimeter via the double polarized Møller scattering of $\vec{e}+\vec{e} \longrightarrow e+e$ with a well known cross section and precisely calculable in QED. In order to relate the beam polarization $P_{b}^{\|}$to the measured polarized cross section we make use of the unpolarized cross section namely:

$$
\frac{d \sigma_{0}}{d \Omega}=\left[\frac{\alpha\left(4-\sin ^{2} \theta\right)}{2 m_{e} \gamma \sin ^{2} \theta}\right]^{2}
$$




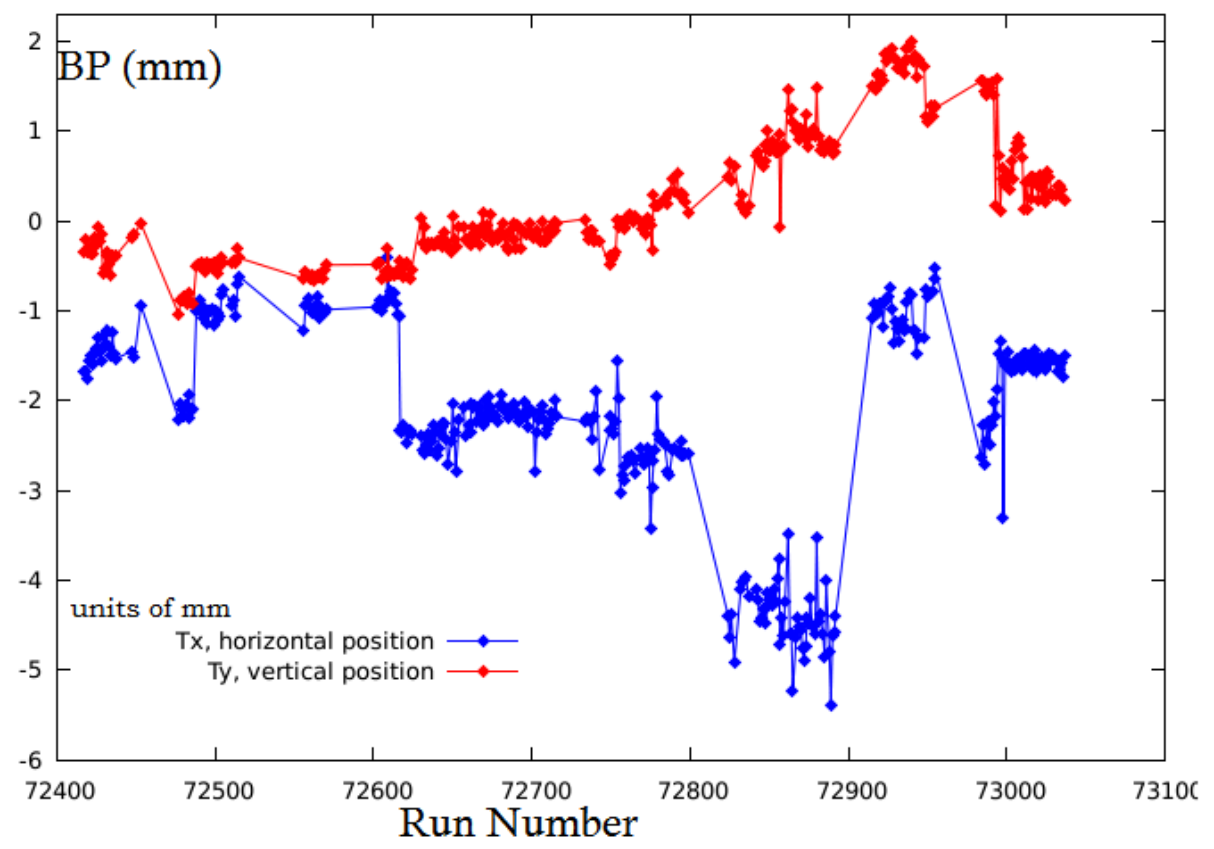

Figure 3.3

Beam position in units of $\mathrm{mm}$ for all the run numbers

where $\theta=$ scattering angle. Hence, the cross section of the above reaction in the center-ofmass frame is:

$$
\frac{d \sigma}{d \Omega}=\frac{d \sigma_{0}}{d \Omega}\left[1+P_{t}^{\|} P_{b}^{\|}(\theta)\right],
$$

where $P_{t}^{\|}$is obtained by polarizing an electron target parallel to the beam axis, $d \sigma_{0} / d \Omega$ is the unpolarized cross section (for the same process), $P_{b}^{\|}$and $P_{t}^{\|}$are the beam and target polarizations respectively. $A_{z z}(\theta)$ known as the analyzing power of the reaction is given by:

$$
A_{z z}(\theta)=-\sin ^{2} \theta \frac{8-\sin ^{2} \theta}{\left(4-\sin ^{2} \theta\right)^{2}}
$$


In term of the asymmetry of the cross section for the beam and target spins parallel and anti-parallel, we obtain:

$$
\epsilon=\frac{d \sigma^{\uparrow \uparrow}-d \sigma^{\uparrow \downarrow}}{d \sigma^{\uparrow \uparrow}+d \sigma^{\uparrow \downarrow}}=A_{z z}(\theta) P_{t}^{\|} P_{b}^{\|}
$$

The beam polarization is thus measured making use of the Møller scattering with a source of polarized target electrons with known polarization. A 4 Tesla field produced by a superconducting split coil solenoid is used to polarize a pure iron film target with the analyzing power maximized for electrons scattered by $90^{\circ}$ in the center of mass frame. In addition, the pairs of electrons are detected in coincidence around this angle.

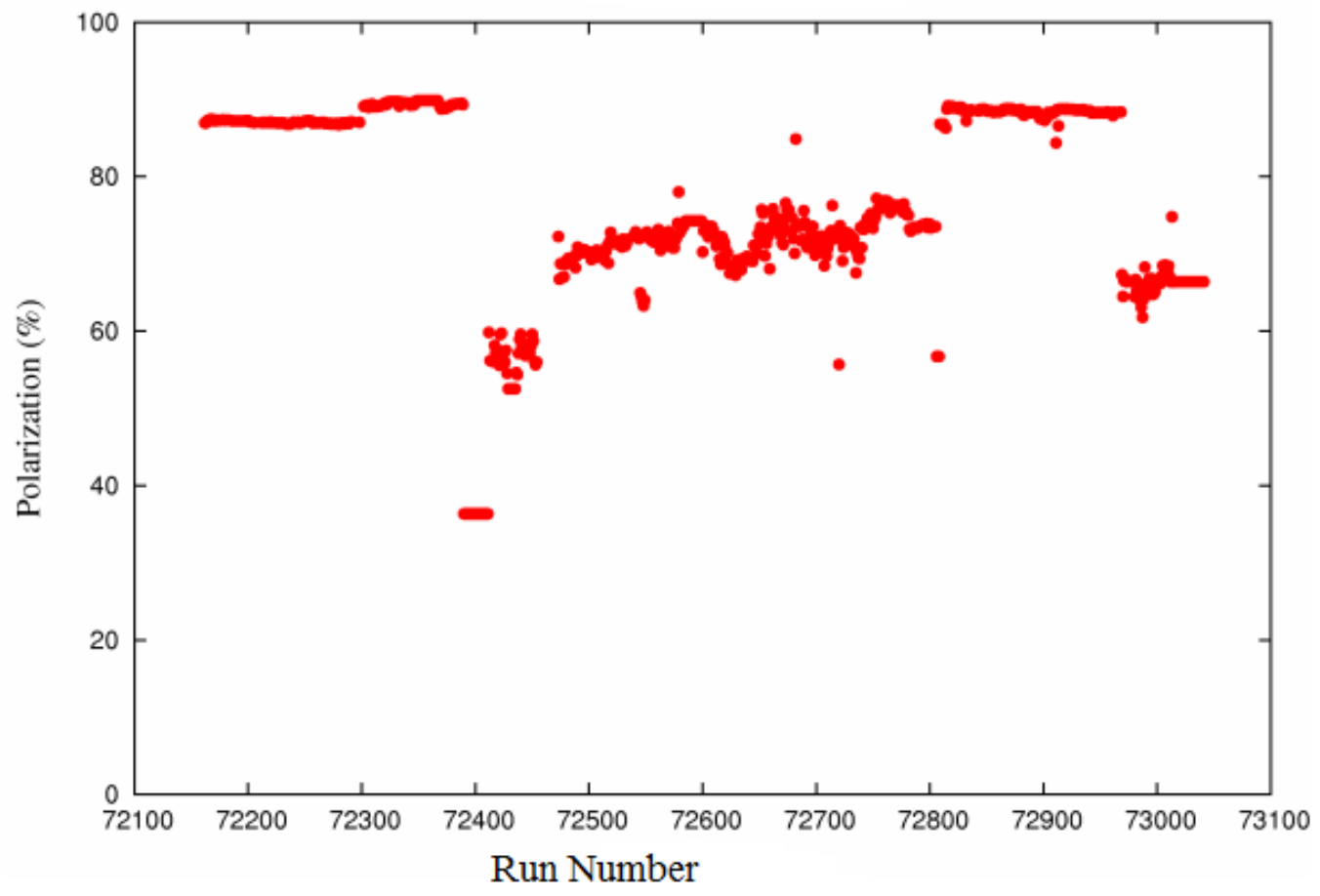

Figure 3.4

SANE's Beam Polarization per Run number 
The background resulting from other scattering processes such as Mott scattering from the iron nuclei are removed due to the coincidence. The scattered electrons undergo a deflection to large angles from a system of two quadrupole magnets. This allows for the analysis of their energy. A system of movable collimators are used for the selection of a narrow range of scattering angle around $90^{\circ}(\mathrm{CM})$.

In all, nine Møller data measurements were done periodically during the SANE experiment [16]. Due to the fact that during an experimental run, the beam polarization is not necessarily that of the most recent Møller measurement. Dave Gaskell, one of SANE's collaborators created a fit to all the good Møller data for the experiment, such that for a given number of passes the beam polarization can be expressed as a function of the beam enegy, wien angle, and the quantum efficiency as reported by the accelerator. The beam polarization measurement of the SANE experiment versus run number is presented in Figure 3.4 .

The jumps in Figure 3.4 are due to the fact that the longitudinal polarized beam was simultaneously delivered to two experimental halls of the three experimental halls (A, B and C). There are over 400 discrete energy combinations which provide longitudinal polarized beam polarization in any two halls simultaneously [47]. Combinations for beam to be delivered to two experimental halls ( $\mathrm{A}$ and $\mathrm{B}, \mathrm{B}$ and $\mathrm{C}, \mathrm{A}$ and $\mathrm{C}$ ) simultaneously, for 5-pass accelerator energies between 2 and $6 \mathrm{GeV}$ are presented in appendix $\mathrm{A}$. 
Using the following physical constants: $m=0.51099906 \mathrm{MeV} / c^{2}$; and $(g-2) / 2=$ 0.001159652 , we can write the total precession from the injector to each of the three halls as:

$$
\begin{aligned}
& \theta_{A}=P\left[2 n_{A}^{2}-n_{A}\left(1-2 \alpha-\frac{1}{2.4}\right)-\alpha\left(1-\frac{1}{4.8}\right)\right] \pi \equiv m_{A} \pi \\
& \theta_{B}=P\left[2 n_{B}^{2}-n_{B}(1-2 \alpha)-\alpha\right] \pi \equiv m_{B} \pi \\
& \theta_{C}=P\left[2 n_{C}^{2}-n_{C}\left(1-2 \alpha+\frac{1}{2.4}\right)-\alpha\left(1+\frac{1}{4.8}\right)\right] \pi \equiv m_{C} \pi
\end{aligned}
$$

Where $P=\left(\frac{E_{1}}{m}\right)\left(\frac{g-2}{2}\right), E_{1}$ is the energy of a single linac, $\alpha=0.1125$ is the ratio of the injector energy to the linac energy, and $n_{A}, n_{B}$, and $n_{C}$ are the number of recirculation passes delivered to the indicated hall. Both linac are assumed to operate at the same energy, and hence the energy of the beam in any particular hall is given by:

$$
E_{A, B, C}=\left(2 n_{A, B, C}+\alpha\right) E_{1}
$$

In order to find the beam energy combinations which will provide simultaneous longitudinal polarization in any two halls, we simply require that the difference in the precessions to the two halls in question be an integral multiple of $\pi$. In these cases, a single orientation of the polarization at the injector can be found which will arrive longitudinally in each of the two halls. The general form of the precession difference equation is:

$$
\left(\frac{\theta_{\sigma}-\theta_{\tau}}{\pi}\right)=\operatorname{Pf}\left(n_{\sigma}, n_{\tau}\right)=m_{\sigma}-m_{\tau}
$$

For each particular choice of two halls, $\sigma$ and $\tau$, there are 21 possible values for the function $f$, the precession difference function which depends only upon the number of the recirculation pass delivered to each of the two halls. These 21 values of $f$ for the cases of 
halls $\mathrm{A}$ and $\mathrm{B}$, halls $\mathrm{C}$ and $\mathrm{B}$, halls $\mathrm{A}$ and $\mathrm{C}$ are given in appendix $\mathrm{A}$. In general, the values of $f$ are large. In general there is a large precession different between any two halls - that permits so many energy combinations to provide simultaneous longitudinal polarizations to these two halls.

\subsubsection{The Raster System}

The Raster system consisted of the Fast and the slow Raster. The slow raster is operated in a $2 \mathrm{~cm}$ maximum diameter spiral pattern. Generally, the raster system is used to spread the beam over the polarized target's surface area uniformly to avoid rapid depolarization due to heating and radiation damage [16]. At Jefferson Lab, the standard fast raster spreads the beam over $2 \mathrm{~mm}$ by $2 \mathrm{~mm}$. The $2 \times 2 \mathrm{~mm}$ Hall $\mathrm{C}$ fast raster is superimposed on the slow raster pattern. The target used in this experiment is a cylinder of frozen ammonia beads with a diameter of approximately $2.5 \mathrm{~cm}$. The target will be discussed in detail later in section 3.5. A single loop coil, about a $1 / 2$ inches in diameter, embedded directly in the target material serves as a pickup for the polarization measurement.

When the beam passes through the target material, it damages it. This damage to the target material causes depolarization over time. Spreading the beam out with a wider raster reduces the radiation induced depolarization rate.

Two deflecting magnets serve to steer the beam. These magnets are controlled by three signal generators to establish a spiral pattern that maintains a constant beam flux over the area it sweeps out. 
This spiral raster pattern fills a circle of diameter $2 \mathrm{~cm}$. The raster current signal are sent to an ADC and read out by CODA. The raster signal data allows for the calculation of the beam position within the raster at any given time. A graph of the target can be produced by plotting the $x$ versus $y$ position of the raster for events in the main detector. More details can be found in [18].

\subsection{Chicane and Helium Bag}

Chicane is the name given to two set of dipole magnets, BE and BZ used to align the beam on the target. The chicane is used because the standard Hall $\mathrm{C}$ beam would be deflected down by the target magnetic field when its at $80^{\circ}$ relative to the beam causing the beam to miss the center of the target and also miss the beam dump in the rear of the Hall C.

When the target is oriented such that its 5T magnetic field is parallel or anti-parallel to the beam line, the trajectory of the beam is unaffected (as we can see from $\vec{v} \times \vec{B}$ ). On the other hand, when the target field is oriented near perpendicular $\left(80^{\circ}\right)$ relative to the beam, the beam is bent downward. Furthermore, SANE required near perpendicular target polarization (hence magnetic field alignment) for most of the experiment so the target was installed and kept for the entire experiment at the same level as the beamline.

The BE dipole magnet was used to bend the incoming beam vertically down toward the BZ, which in turn bent the beam back up at the target. These magnets were precisely positioned to allow the beam to hit the center of the target after being bent by the target 


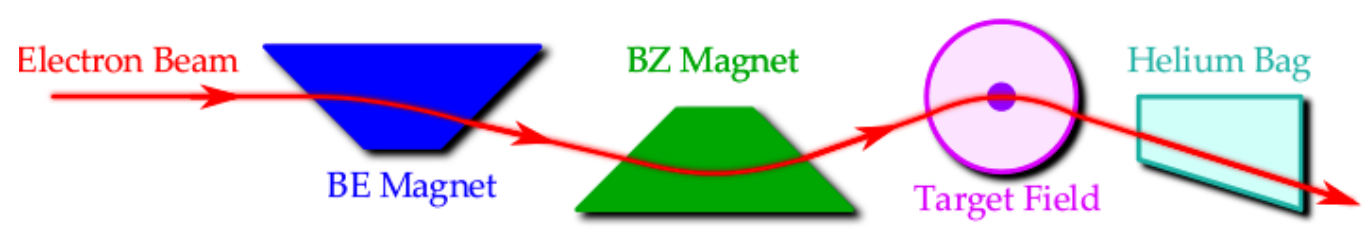

Figure 3.5

SANE Beamline During Perpendicular Target Field Running

magnetic field. The magnetic field setting for the chicane is tabulated in Table 3.2 [32].

Figure 3.5 shows SANE beamline during perpendicular target field running.

Table 3.2

Chicane Setting

\begin{tabular}{cccc}
\hline Beam Energy $(\mathrm{GeV})$ & $B E \int B d l$ & $B Z \int B d l$ & Target $\int B d l$ \\
\hline 4.7 & $1.002 \mathrm{Tm}$ & $0.513 \mathrm{Tm}$ & $1.521 \mathrm{Tm}$ \\
5.9 & $1.002 \mathrm{Tm}$ & $0.519 \mathrm{Tm}$ & $1.521 \mathrm{Tm}$ \\
\hline
\end{tabular}

The helium bag is used to prevent background and to handle the beam transport to the beam dump. That is, to prevent hazardous ionization and activation of the air in the hall as the beam passing through. The beam passing through the hall must be shielded from the surrounding atmosphere. This was done by using an 80 -foot-long helium bag which consisted of a 0.04 inch of aluminum windows at the entrance on an extension piece and at the exit to the beam dump for both when the beam is running straight through and when it is bent. Mississippi State University contributed in the downstream beamline design and the helium bag. 


\subsection{Target}

SANE made use of a frozen ${ }^{14} \mathrm{NH}_{3}$ as a proton target prepared via dynamic nuclear polarization (DNP) in a 5 Tesla magnetic field around $1 \mathrm{~K}$. This was done by the University of Virginia Solid Polarized Target group. This section aims to describe in detail the target preparation.

\subsubsection{Why Ammonia}

Ammonia was ideal for the following reasons: it has good reproducibility of the target, contains paramagnetic radicals, yields high maximum proton polarization, exhibit short polarization buildup time and is a more radiation-resistant material than other compounds. In addition, the radiation damage can be repaired by annealing. The process of annealing allows the recombination of paramagnetic centers to restore polarization. In other words, to anneal, the target material is moved out of the beam and the polarizing microwave radiation and is heated to between $90-100 \mathrm{~K}$ for about 30 minutes.

\subsubsection{Dynamic Nuclear Polarization DNP}

The polarization mechanism in DNP is used to obtain a high polarization of the nuclear spins. This is accomplished by the utilization of a microwave field in a high magnetic field to transfer the polarization of the free electron spin in the medium to the nucleon. This method was first developed in 1953 for metals [29] and in 1958 for solid insulators $[30,31]$. The various mechanisms that contribute to the DNP process shall be examined in this subsection. 


\subsubsection{Thermal Equilibrium Polarization}

In order to polarize a particular material such as a proton, the interaction of the magnetic moment of the particles of interest with an external magnetic field is generally a vital kickoff point. Consider a polarized target to be an ensemble of such particles placed in a high magnetic field and cooled to low temperature. A magnetic moment $\vec{\mu}$ in the external field, $\vec{B}$ establishes a set of $2 I+1$ energy sublevels due to the Zeeman interaction, where $I$ is the spin angular momentum. According to Boltzmann distribution, the relationship of the populations of two states for a spin $\frac{1}{2}$ particle is given by:

$$
N_{1}=N_{2} \cdot \exp \left(\frac{-\Delta E}{K_{B} T}\right)
$$

Where $N_{1,2}$ are the population numbers of the sublevels, $T$ is the temperature of the system, and $K_{B}$ is the Boltzmann constant. Given that the Zeeman interaction takes the form

$$
\vec{\mu} \cdot \vec{B}
$$

this implies that, for a spin $\frac{1}{2}$ particle, the ratio of the number of particles in the aligned state to anti-aligned state is given as:

$$
\frac{N_{\uparrow}}{N_{\downarrow}}=\exp \left(\frac{-\mu B}{K_{B} T}\right)
$$

Where $N_{1}$ and $N_{2}$ become $N_{\uparrow}$ and $N_{\downarrow}$; the number of particles in the aligned and antialigned states respectfully.

The vector polarization of the material, $P$, is a measure of the particle's spin alignment in the magnetic field and is typically defined as:

$$
P=\frac{N_{\uparrow}-N_{\downarrow}}{N_{\uparrow}+N_{\downarrow}}
$$


substituting equation (3.13) into equation (3.12), we get:

$$
P=\frac{e^{\frac{\mu B}{K T}}-e^{\frac{-\mu B}{K T}}}{e^{\frac{\mu B}{K T}}+e^{\frac{-\mu B}{K T}}}=\tanh \frac{\mu B}{K T}
$$

However, the magnetic moment of the proton, $\mu_{p}$ is small hence, the nucleon polarization obtained in this manner is very small.

For a $2.5 \mathrm{~T}$ magnetic field, we deduce using equation (3.14) that the electron polarization is approximately $92 \%$ at $1 \mathrm{~K}$. Since the magnetic moment of the proton is much smaller than that of the electron $\left(\mu_{e} \approx 660 \mu_{p}\right)$, this results in a much lower proton polarization of $0.25 \%$ at $2.5 \mathrm{~T}$ and $1 \mathrm{~K}$ [53]. Clearly, since producing magnetic fields much greater than $2.5 \mathrm{~T}$ and temperatures much below $1 \mathrm{~K}$ are difficult to achieve, there is a need to seek for other methods to achieve high proton polarization.

\subsubsection{Solid-State Effect}

As concerns the solid-state effect; the polarization of a desired target material with a high concentration is accomplished via doping with paramagnetic radicals which provide the unpaired electron spins.

The electron polarization is very high since the magnetic moment of the electron is much larger than that of the nucleon. Furthermore, contact between both spin species is provided as a result of hyperfine splitting. Hyperfine splitting is caused by the dipole-dipole interaction between the nucleon and the electron spins. With a frequency of about $140 \mathrm{GHz}$ at $5 \mathrm{~T}$, very close to the electron spin resonance frequency, the high electron polarization can be transferred to the proton. More so, the relaxation time for the nucleon spin is much longer than for the electron spin. Different values of the frequency are employed to align 


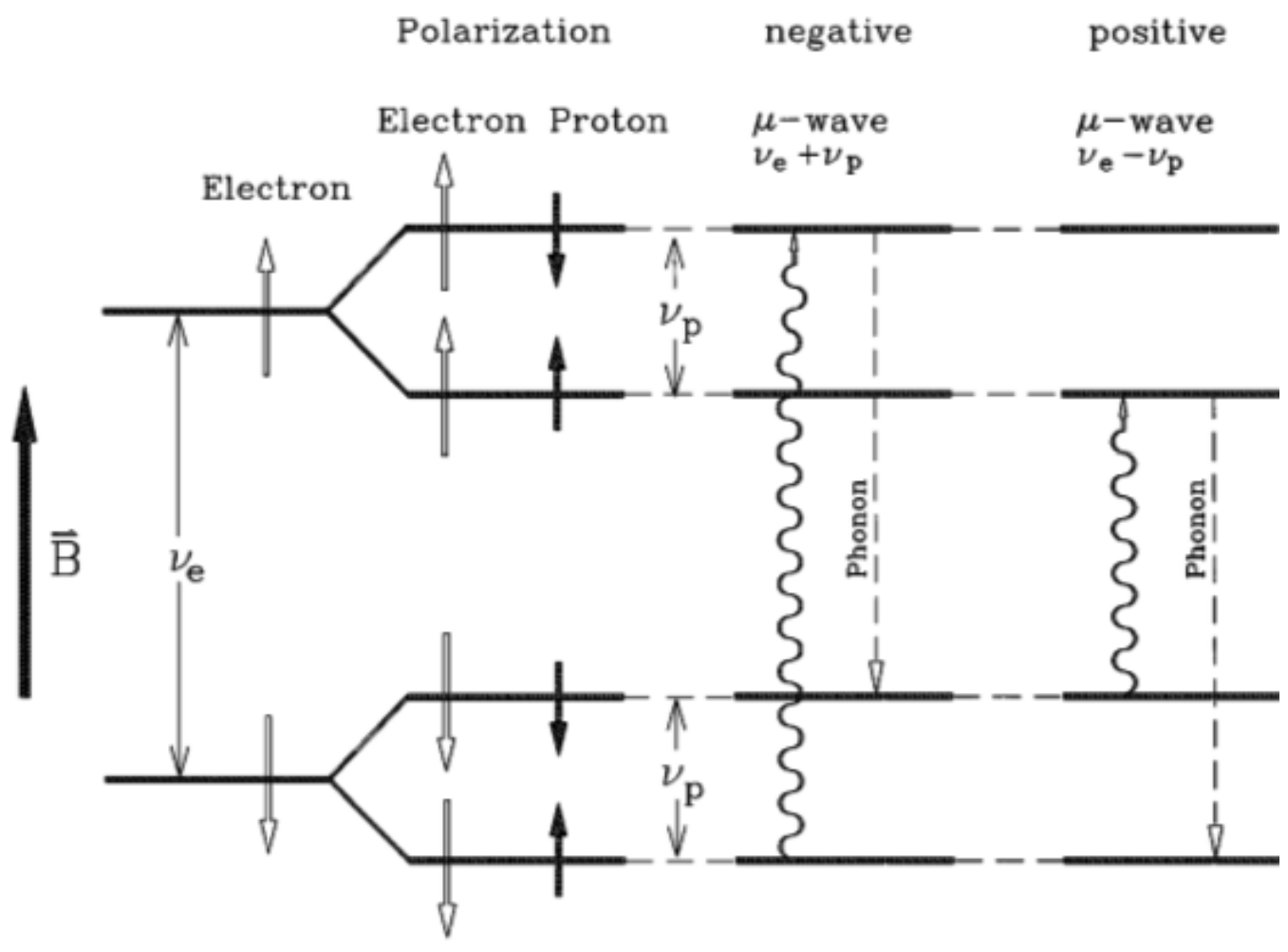

Figure 3.6

Schematic Demonstration of the Solid-State Effect 
the nucleon polarization either parallel or anti-parallel to the applied magnetic field as shown in Figure 3.6 [53]. It can also be shown quantum mechanically that the Hamiltonian in solid-state effect contains solely of the Zeeman interactions of the nucleon spins and the electron spins

$$
H=\overrightarrow{\mu_{e}} \cdot \vec{B}+\overrightarrow{\mu_{p}} \cdot B+H_{s s}
$$

where $H_{s s}$ is the spin-spin interaction term

In this model, one of the forbidden transitions $\left(\nu_{e} \pm \nu_{n}\right)$ is assumed to be excited provided the electron spin resonance (ESR) spectrum of the paramagnetic radicals is narrow compared to $\nu_{n}$. Basically, the population numbers of the dynamic equilibrium depend on the following: the line width, $\Delta \nu_{e}$, the relaxation times of the electrons, and the electron-nucleon coupling. Furthermore, these values determine the range of temperature and magnetic field in which dynamic polarization is feasible. The maximum polarization also depends on the microwave intensity. However, processes involving relaxation are disadvantaged because of paramagnetic impurities, that is radicals that do not contribute to the buildup of nucleon polarization.

\subsubsection{Equal Spin Temperature Theory}

In current polarization target materials, in which the radicals are introduced by irradiation, the process of dynamic polarization is somewhat different from the solid-state effect and is described by the theory of Equal Spin Temperature (EST) [53]

In the situation where the concentration of the electrons is high, the dipolar interactions

of the electron spins can no longer be neglected. These interactions with non discrete 

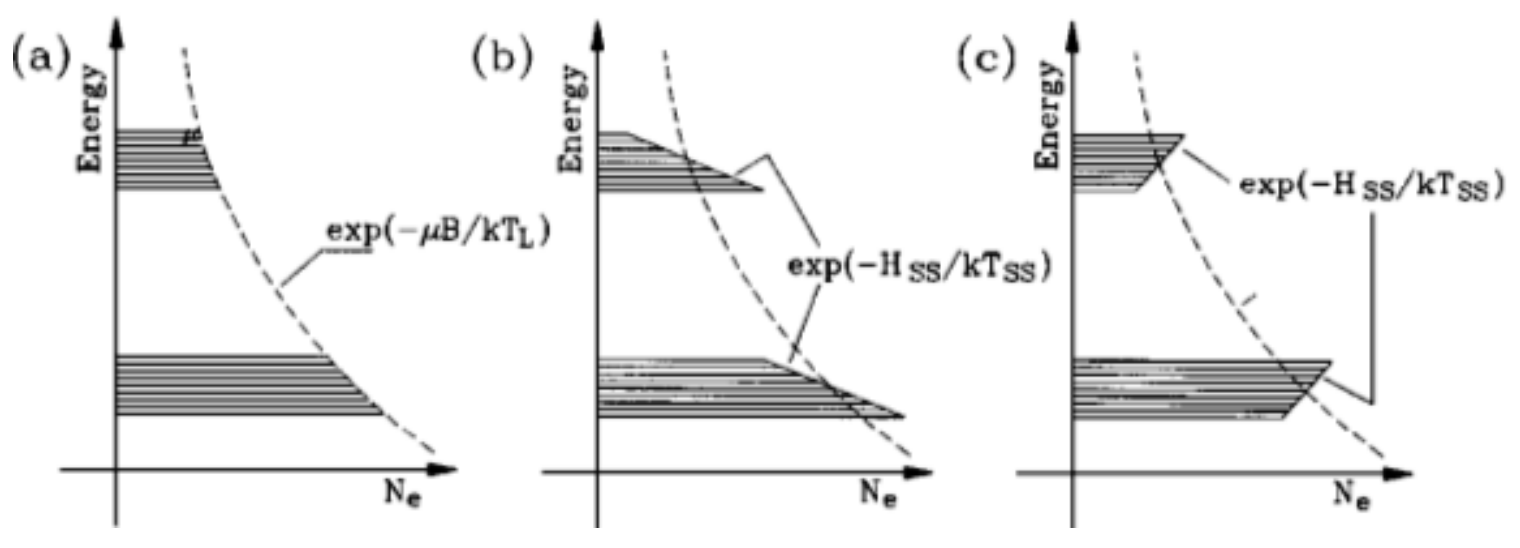

Figure 3.7

Population Densities of the Electron Levels

energy levels are weaker than to the Zeeman interaction of the electron spins. With no energy levels for equal spin quantum numbers, it becomes exceedingly difficult to describe the time evolution of the system under the influence of a microwave field or spin lattice relaxation acting as a perturbation.

With such an energy band, the population of the states inside and that of different bands are described by Boltzmann distribution with the temperatures, $T_{S S}$ and $T_{Z_{e}}$ being the temperatures of the electron spin-spin interaction reservoir (SSI-reservoir) and the electron Zeeman reservoir, respectively. As depicted in Figure 3.7 (a), $T_{S S}$ and $T_{Z_{e}}$ are identical to the lattice temperature $T_{L}$ only at thermodynamic equilibrium. The energy of the SSIreservoir is characterized such that it can also have negative values (Figure 3.7 (b), (c)) [16].

This implies that the upper energy population levels inside a band is higher than the population of the lower ones. In the case of a different $T_{S S}$ at constant $T_{Z_{e}}$, this implies 
that on average the number of spins in the magnetic field direction is constant but the spatial

distribution is not. DNP in the spin temperature mechanism can be described in two steps:

1. Cooling of the electron SSI-reservoir: In the first step, a quantum with the energy $h\left(\nu_{e}-\Delta\right)$ is absorbed from the microwave field. The energy is divided into two parts, one part $h \nu_{e}$ that is used to change the electron Zeeman energy while the other part, $h \Delta$ is absorbed by the electron SSI-reservoir. On the one hand, if $\Delta>0$, the SSI-reservoir emits this energy and cools down; on the other hand if $\Delta<0$, the SSI-reservoir is heated and $T_{S S}$ may become negative as shown in Figure 3.7.

2. The second step is the proper thermal mixing process whereby there is heat contact between the electron SSI-reservoir and the Zeeman reservoir of the nucleon. In this case, a forbidden relaxation process is considered that consists of a flip-flop of two electron spins together with a flip of nucleon spin. However, the electron-Zeemanreservoir energy is unaffected, whereas that of the nucleon-Zeeman-reservoir changes by $h \nu_{n}$ and this energy is exchanged between both reservoirs. Thus $T_{S S}$ and $T_{Z_{n}}$ are equalized.

This process has been seen in frozen ammonia where nitrogen polarization occurs during the exchange of transitions to polarize the hydrogen nuclei. It can be shown that for any body with a magnetic moment $g_{i} \mu_{i}$ in the material, the polarization for a spin $\frac{1}{2}$ particle is given by:

$$
P=\tanh \frac{g_{i} \mu_{i} B}{2 K_{B} T_{S S}}
$$

where $T_{S S}$ is the temperature of SSI-reservoir. Therefore, in ammonia, if the proton's polarization is determined, the spin spin temperature can be computed and the nitrogen nucleus polarization obtained.

The EST theory has been experimentally confirmed in ${ }^{14} \mathrm{NH}_{3}$ (Figure ??) where ${ }^{14} \mathrm{~N}-\mathrm{H}$ system shows equal spin temperatures during the DNP pumping process [16].

\subsubsection{Target Cryogenic}

The target used was a polarized ammonia target. The magnetic field for polarizing the target is a superconducting magnet built in the Helmholtz configuration with a cyrostat that serves as a liquid helium supply for the target refrigeration system. The ${ }^{4} H e$ evaporation 
refrigerator was used to cool the target, operating at around $1 \mathrm{~K}$ with a cooling power of about $1.5 \mathrm{~W}$ in a $5 \mathrm{~T}$ field.

This cooling power is important to remove heat from the beam and microwaves used for DNP. For the best polarization performance, it is necessary to operate the refrigerator with a high magnetic field [53]. DNP works best when the magnetic field is $\frac{B}{T_{L}} \approx 5-10 \mathrm{~T}$ where $T_{L}$ is the lattice temperature of the material. Figure 3.8 shows a picture of the UVa target that was used during the SANE experiment.

The target material is enclosed in an insert (see Figure 3.9) which extends into the nose of the refrigerator, where cooling is provided by liquid helium at $1 \mathrm{~K}$. The supply of helium is from the magnet helium reservoir through an insulated jumper and flows through baffles which cool the liquid. Liquid helium flows through the separator plate into a heat exchanger and into the target holder via a needle valve. The pool of liquid in the target holder is pumped on by large capacity roots pumps to reduce the temperature to less than $1 \mathrm{~K}$. As the cold vapor is pumped away, it exchanges heat with and cools the incoming warm liquid. Services like the microwaves, the data acquisition electronics, and the NMR (Nuclear Magnetic Resonance) are also brought into the target cavity to provide an online of the target polarization and recorded operating condition.

The target material was inserted such that it suspended in the magnet's uniform field region in the refrigerator's nose by the target insert. The target ladder is around $1.5 \mathrm{~m}$ long and provides storage for two target material samples in $2.5 \mathrm{~cm}$ diameter target cups at the bottom. The target ladder consisted of, two target cavities, carbon disk and tungsten wire cross-hairs. In addition, it carries semi-rigid cable down to the NMR coils inside the target 


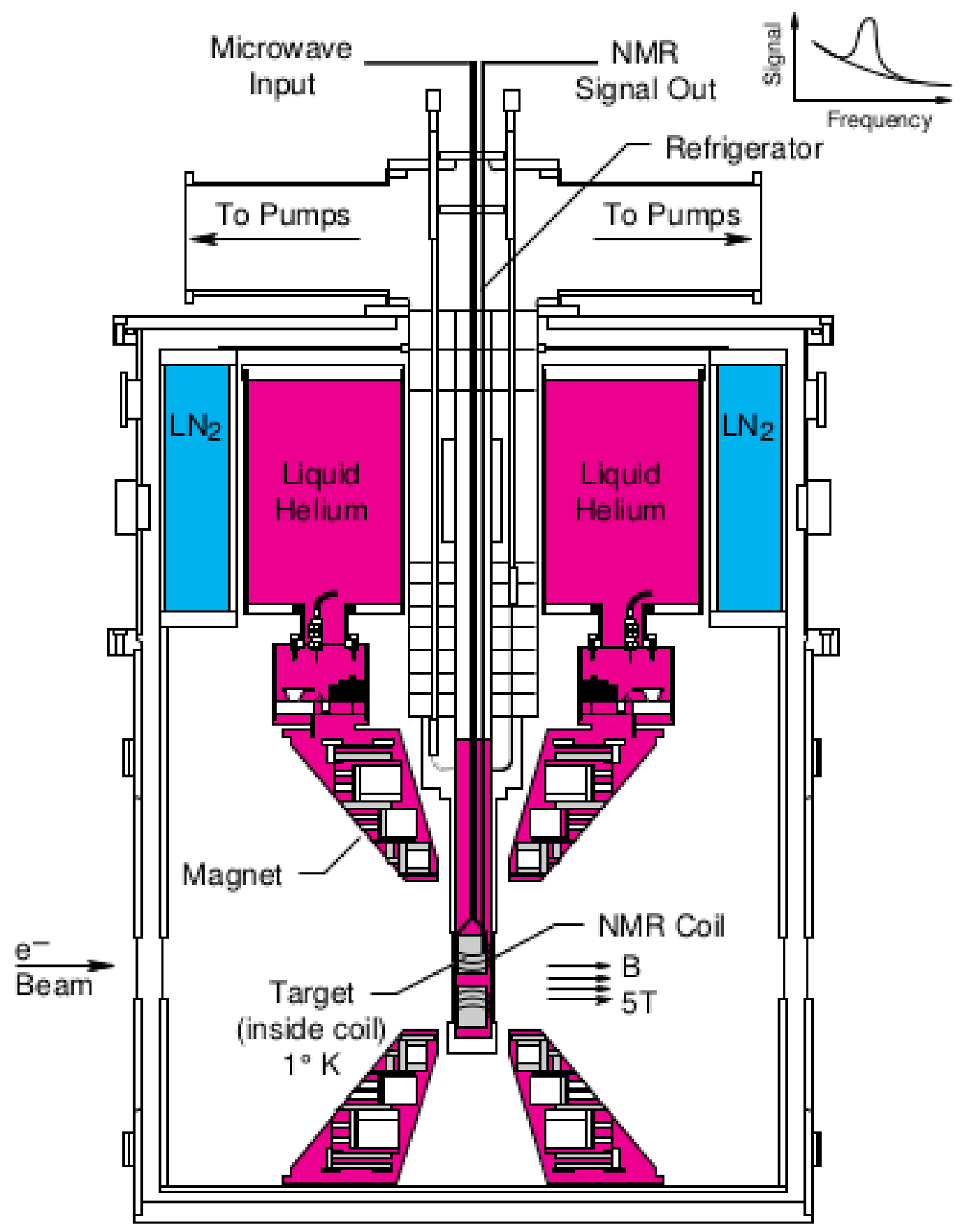

Figure 3.8

Target and magnet used for SANE 
cavities, and microwave guides and extend down to horns on each of the target material cup.

Since anneals (allowing the recombination of paramagnetic centers to restore polarization) of the material require precise temperature data, the ladder is equipped with, thermocouples, platinum resistors and carbon-glass resistors. Heater wire runs to the bottom of the ladder to provide the heat needed to perform anneals, and the entire ladder was raised and lowered by a mechanized lift to position the correct target cup in question in the beam.

\subsubsection{Microwaves}

The microwaves needed to drive the polarization enhancement in DNP were supplied by an Extend Interaction Oscillator (EIO) [16]. The EIO was located above the target during the experiment, coupled to either target material cups by a switching junction and over-sized CuNi wave-guides broadcast microwaves evenly over the cups. The EIO tube has a frequency of about $28 \mathrm{GHz} / \mathrm{T}$, i.e., $140 \mathrm{GHz}$ at $5 \mathrm{~T}$ and can be tuned using a mechanical bellows by $\pm 2 \mathrm{GHz}$ in order to drive the positive or negative polarization transition. The microwave frequency and power were monitored during the running of the experiment by a target operator. The frequency at which optimal polarization is achieved shifts while the polarization is building up and while the beam is being applied to the target. The local field around the nuclei and free radicals is affected by the free radical density. The density changes as the beam damages the target material. The changing local field causes a shift in optimal microwave frequency for polarization. It is necessary to monitor the polarization and continually adjust the microwave frequency in order to maintain maximum 


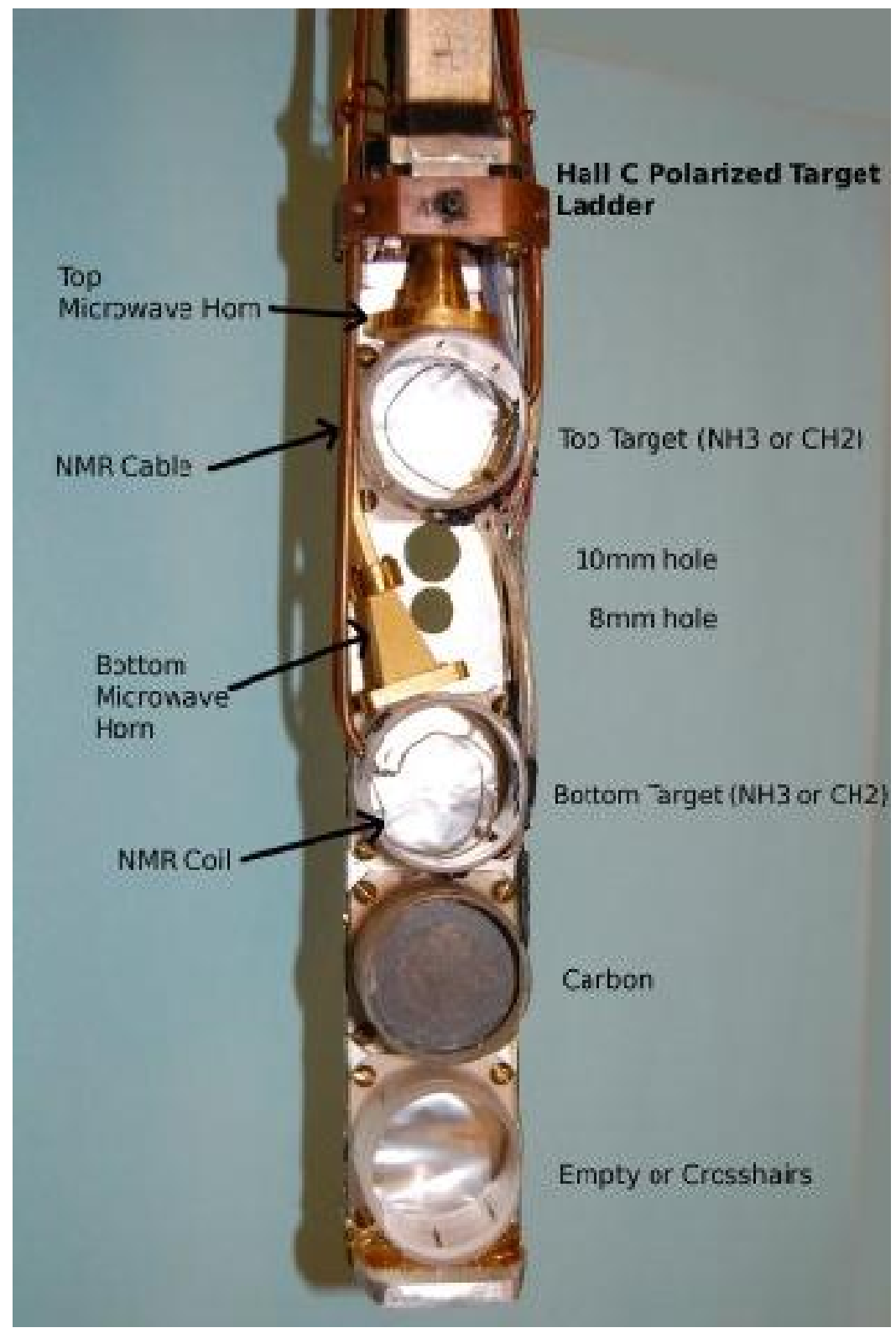

Figure 3.9

Target Ladder 
polarization. An adjustment of a few megahertz every half hour was sufficient to prevent unnecessary decay in polarization.

\subsubsection{Nuclear Magnetic Resonance and Polarization}

A spin- $I$ system placed in a magnetic field $\vec{B}$ shows a Zeeman energy splitting into $2 I+1$ levels. These levels are separated in energy by

$$
\hbar \omega_{L}=\frac{\mu \overrightarrow{\vec{B}}}{I}=g \mu_{n} B,
$$

where $g$ is the $g$-factor of the particle with spin $I$ and $\mu_{n}$ is the nuclear magneton. When the spin system is irradiated by an $r f$ field at the Larmor frequency, the spin system either absorbs some energy or the $r f$ induces the spins to emit energy. The response of a spin system to $r f$ irradiation is described. by its magnetic susceptibility

$$
\chi(\omega)=\chi^{\prime}(\omega)-i \chi^{\prime \prime}(\omega)
$$

where $\chi^{\prime}(\omega)$ is the dispersive and $\chi^{\prime \prime}(\omega)$ the absorptive part of the susceptibility. The absolute polarization of the material is proportional to the integral of the absorptive part of the susceptibility [53].

$$
P=K \int_{0}^{\infty} \chi^{\prime \prime}(\omega) d \omega
$$

where $K$ is a constant containing the properties of the NMR system concerned.

The polarization is measured by means of the nuclear magnetic resonance (NMR) method, using a series $Q$-meter as shown in Figure 3.10. The $Q$-meter is connected to an NMR-coil with inductance $L_{c}$ and resistance $r_{c}$, that is embedded in the target material 


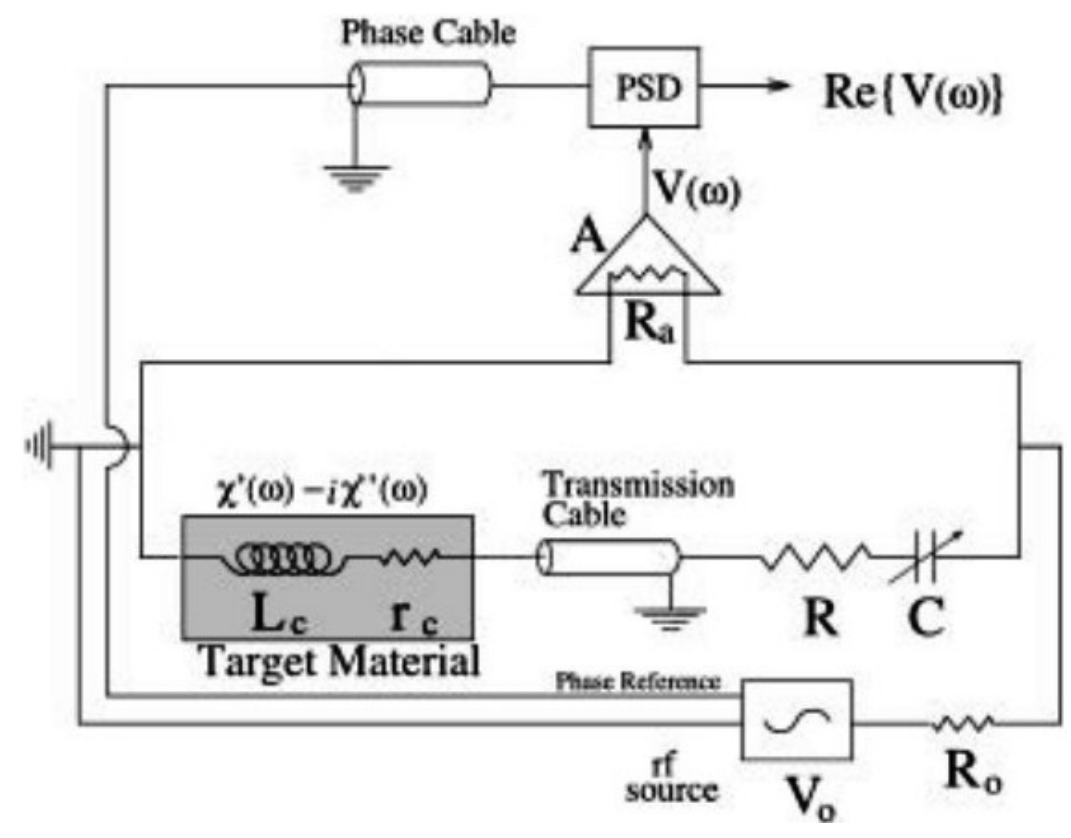

Figure 3.10

Schematic Drawing of the $Q$-meter (NMR) Circuit

via coaxial transmission cable, capacitor $C$, and damping resistance $R$ that forms a series $L R C$ circuit.

Due to inductive coupling between the spins and the coil, the impedance of the coil becomes

$$
Z_{c}=r_{c}+i \omega L_{c}(1+4 \pi \eta \chi(\omega))
$$

where $\eta$ is the filling factor of the coil. As shown in Figure 3.10, the circuit is driven by a frequency synthesizer, $V_{0}$, which sweeps the $r f$ frequency $\omega$ through the Larmor resonance. This causes a change of the inductance of the coil as the target material absorbs or emits energy. With the current kept to a constant, the inductance change in turn causes an inductance change in the circuit, which is proportional to the complex output voltage 
$V(\omega, \chi)$. At the last stage, a phase sensitive detector (PSD) allows the selection of the real part of the voltage by using the input $r f$ signal as a reference. The voltage is a superposition of both the signal, proportional to $\chi$, and the $Q$-curve. The $Q$-curve can thus be separated out by measuring the circuit response with the Larmor frequency shifted outside the range of the modulation by lowering the magnetic field. With the measurement of the $Q$-curve, it can be subtracted out and the result integrated as in equation (3.19) with $K$ the calibration constant that depends on the state of the NMR circuit. This calibration constant can be measured by doing a polarization measurement while the material is at a known polarization.

The NMR system is used to measure the material about every thirty seconds during the production runs. The frequency modulated signal of the coils in the material have a central frequency of $213 \mathrm{MHz}$ (Where $213 \mathrm{MHz}$ is the Larmor frequency of a proton in a $5 \mathrm{~T}$ field) and a linear sweep range of $400 \mathrm{MHz}$ on either side. The NMR output signal is the sum of the Q-curve and the frequency dependent response of the circuit due to the polarized target material. Figure 3.11 shows the $Q$-curve; the raw NMR signal from which the $Q$-curve is subtracted, and the polynomial fit to the signal ends [17]. During the run the signal can gain a dc-offset which may alter the $Q$-curve slightly due to temperature changes in circuit, thus the need for a polynomial signal.

In order to process the average signal from a $30 \mathrm{~s}$ sweep, the baseline is subtracted, a fit is performed to the ends of the signal, and the resulting curve is subtracted from the signal to remove any DC offset or slight changes to the $Q$-curve. Polarization measurements are taken on intervals of about $30 \mathrm{~s}$ to $60 \mathrm{~s}$ depending on the noise in the signal. For the 


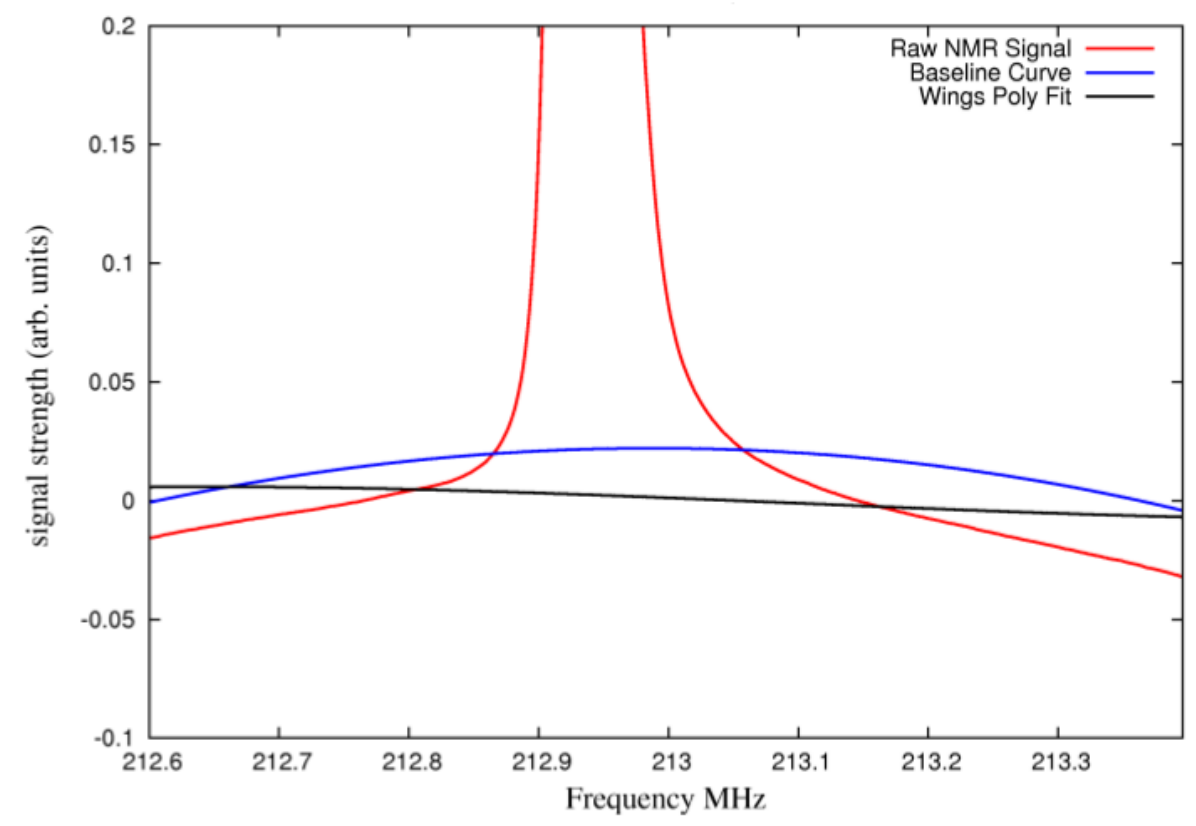

Figure 3.11

NMR signal decomposition

purpose of the analysis, a beam charge weighted average of the polarization is taken for each run. Data was taken from [66] to come up with the plot. The run is then assigned a target polarization value equal to that average. When including data in the average, a cut on the beam current was placed at $60 \mathrm{nA}$. Figure 3.12 show the charge average target polarization for each run. The average for the entire run period is $68 \%$.

\subsubsection{Radiation Damage and Lifespan of a Target Load}

Radiation damage occurs as a result of additional radicals in the target materials created through spallation and ionization during irradiation by the beam. This damage inhibits DNP process and lowers the polarization. These additional radicals do not contribute to the DNP process. With the increase in radical density, the nucleon relaxation time is shortened 


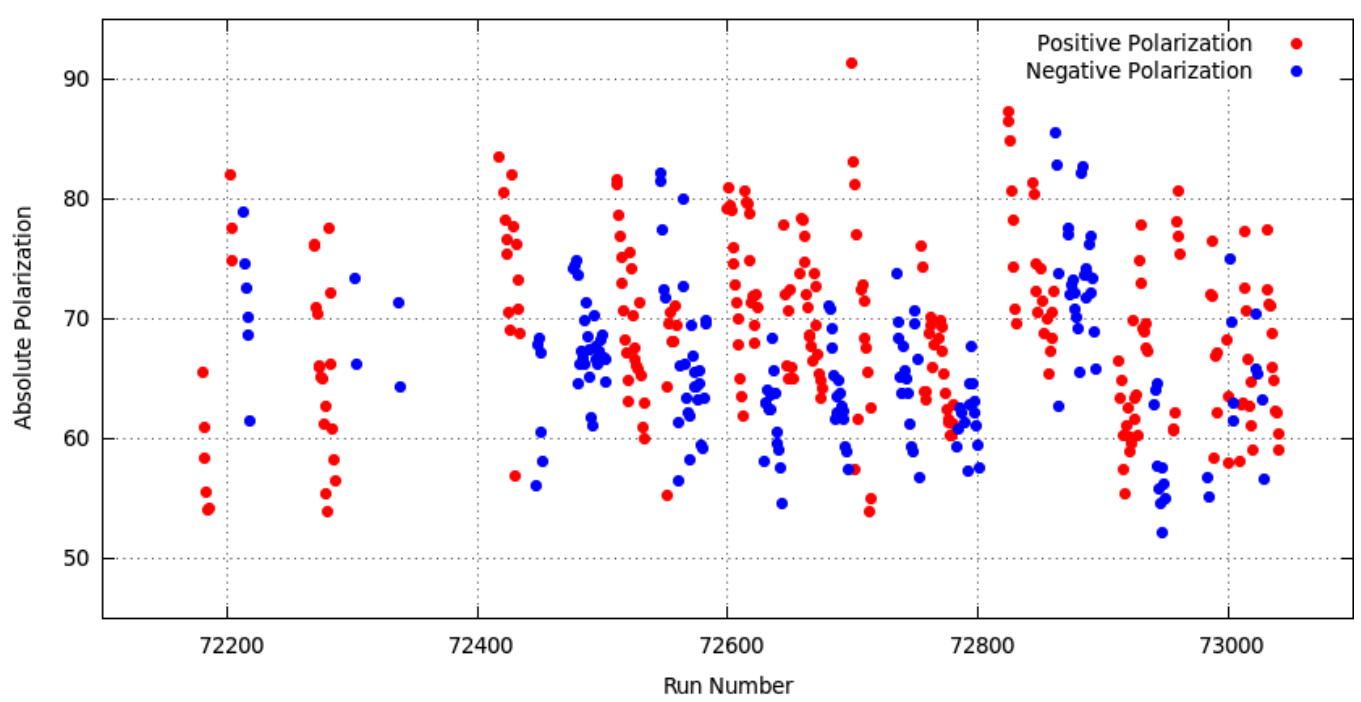

Figure 3.12

Charge averaged target polarization per run for SANE

and the polarization reduced. However, this reduction can be recovered by heating or annealing the target material. During an anneal, the temperature of the material is raised to slightly above liquid nitrogen temperature. Heating the material for about 30 minutes at $90-100 \mathrm{~K}$ decreases the inhibiting radical density and reduces the relaxation rate of the protons in the material, hence raising the possible maximum polarization back to its initial value. This results in a slightly increased depolarization rate per charge. Thus, the material has to be replaced as soon as the depolarization rate is high enough to cause the time cost of more frequent anneals to be greater than the time cost of uninstalling the insert from the fridge, swapping in new materials and reinstalling the insert. More details on radiation damage and annealing can be found in [16] and [53].

The target polarization decreased continually while the electron beam is on thus causing interference with the polarization process as a result of the beam heating. During 
beam trips, the target temperature caused the polarization to change. The microwaves were tweaked by hand as the optimal polarization frequency changed with the radiation dose on the target. There were also instances of gradual rise or unexpected decrease of the target polarization during run period, this could be attributed to sub-optimal tweaking of the microwaves. In addition, loss of helium in the target caused the polarization of the material to drop off suddenly. Therefore, the polarization is immediately destroyed during the beam and microwave heating in the absence of a refrigerant.

Furthermore, there were a series of target magnet failures which delayed the running of the experiment. On the whole, these shortcomings were sufficiently contained thanks to the Hall C management and the UVa target group. Details of the UVa target magnet failure, repairs, cause of damage, behavior after repair can be found in [16].

\subsection{Electron Detector Package}

The electron detector package otherwise known as "BETA", short for for Big Electron Telescope Array is comprised of four components namely: BigCal Pb glass calorimeter, lucite hodoscope, Cerenkov, and a forward tracker. BETA was well suited for the job, since it was non-magnetic with large acceptance, high pixelization, high background rejection and low deadtime with adequate energy resolution to detect DIS electrons. Figure 3.13 shows a photograph of BETA in the experimental Hall C. Figure 3.14 shows an annotated picture of BETA with DIS electron simulation [56]. 


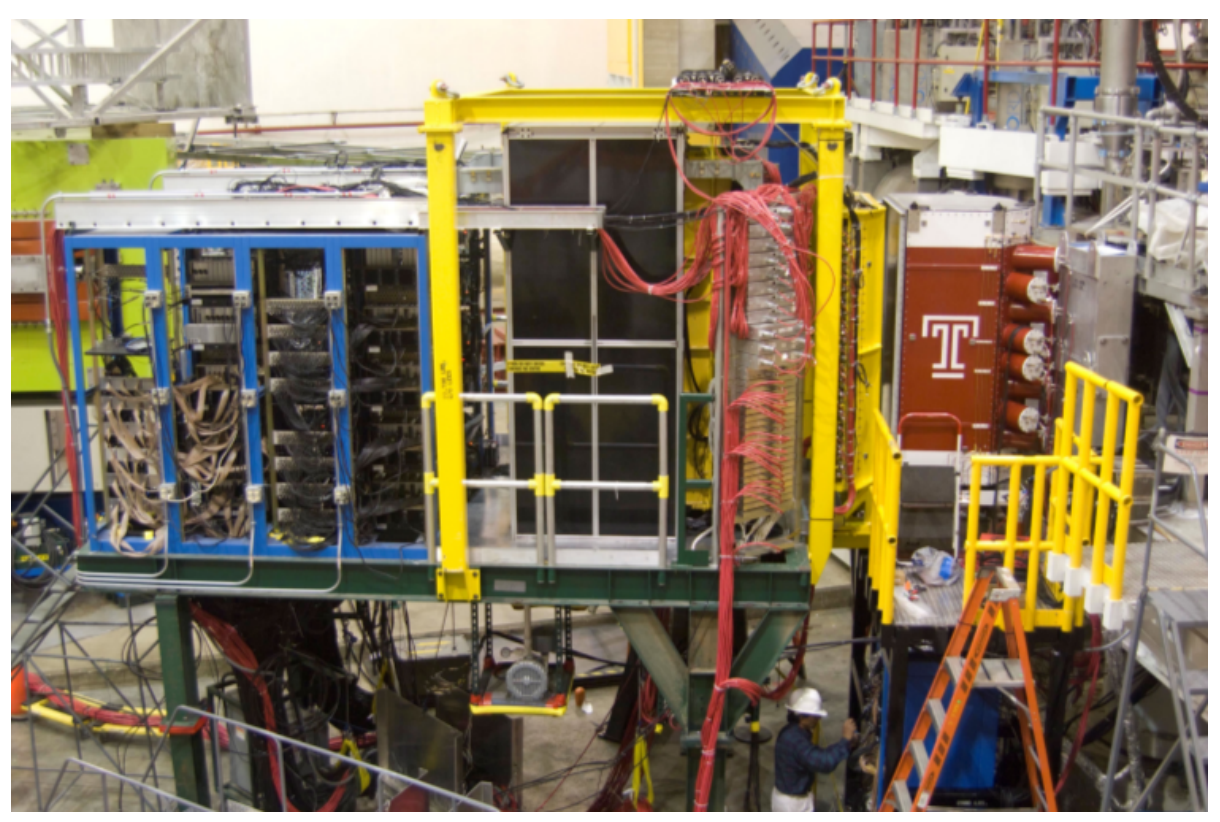

Figure 3.13

Charge averaged target polarization per run for SANE

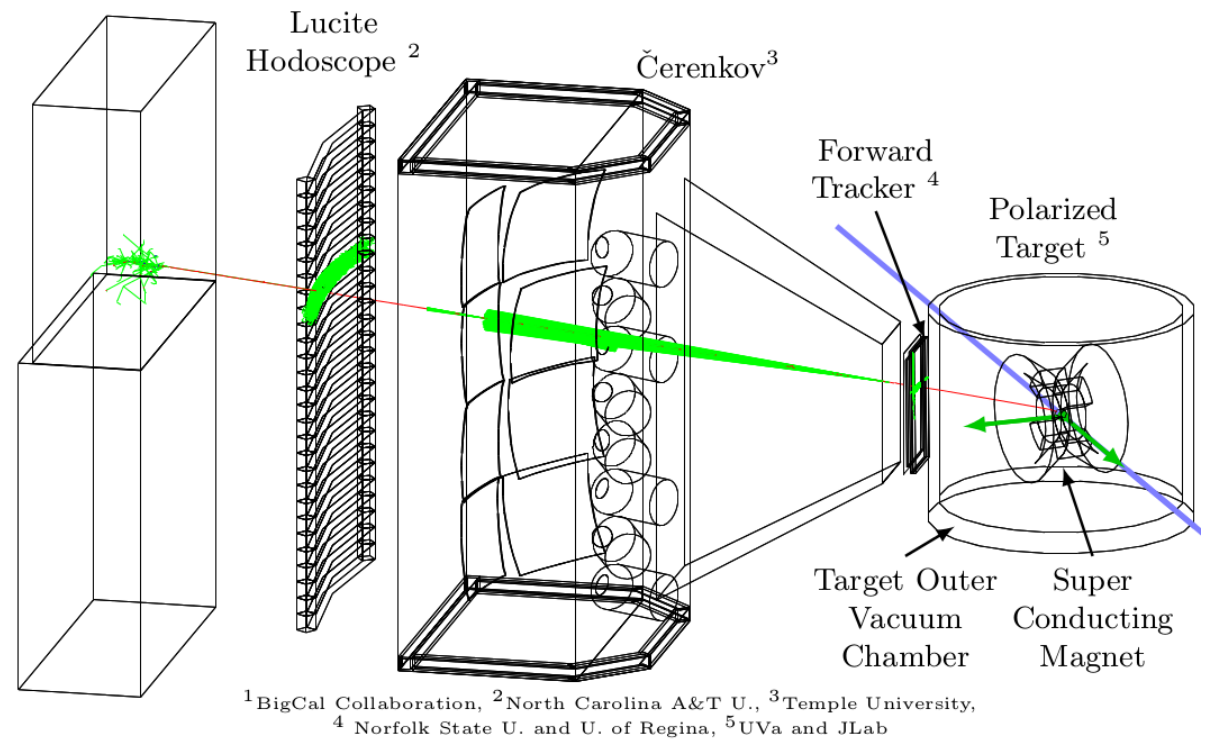

Figure 3.14

Annotated Picture of BETA with DIS Electron Simulation 


\subsubsection{The BigCal Electromagnetic Calorimeter}

The electromagnetic calorimeter consisted of $32 \times 32$ blocks of one dimension of $4 \times$ $4 \mathrm{~cm}^{2}$ of lead glass and $24 \times 56$ blocks of a different size. Each phototube signal was fed into a first level summing module which accepted 8 inputs. Each first level trigger sums 8 phototubes and each row is divided into 4 first level summing modules. These sections of rows are labels A, B, C and D in Figure 3.15. There are 224 first level summing modules. In the module, the 8 individual inputs are amplified 5 times and output individually in the back of the unit. These amplified individual signals go to an ADC. Furthermore, the module sums the 8 inputs and produces 6 summed output signals. One output signal went to a discriminator and then a TDC. Another output channel went to the input of the second level summing module to be summed with the output of 7 other first level summing modules. The second level summing module does not amplify the individual input signals and produces 6 summed output signals. The output of the second level summing module is the sum of 64 photo-tubes. The picture on the right of Figure 3.15 shows an example of the trigger logic where the second level summing module sums the signal from sections A and B (columns 1-16) for rows 4 through 7. This continues for the right half of BigCal and the same is done for the left half. There is no overlap between the halves of BigCal, which leads to a slight loss in trigger efficiency. A total of 39 second-level summing modules were used to cover BigCal. Each individual output signals was sent to a discriminator and the "OR" of the 39 signals was BigCal trigger. Furthermore, the BigCal detector was at $335.4 \mathrm{~cm}$ from the target. Details about the electromagnetic calorimeter can be found in the following references [16, 17, 32]. 

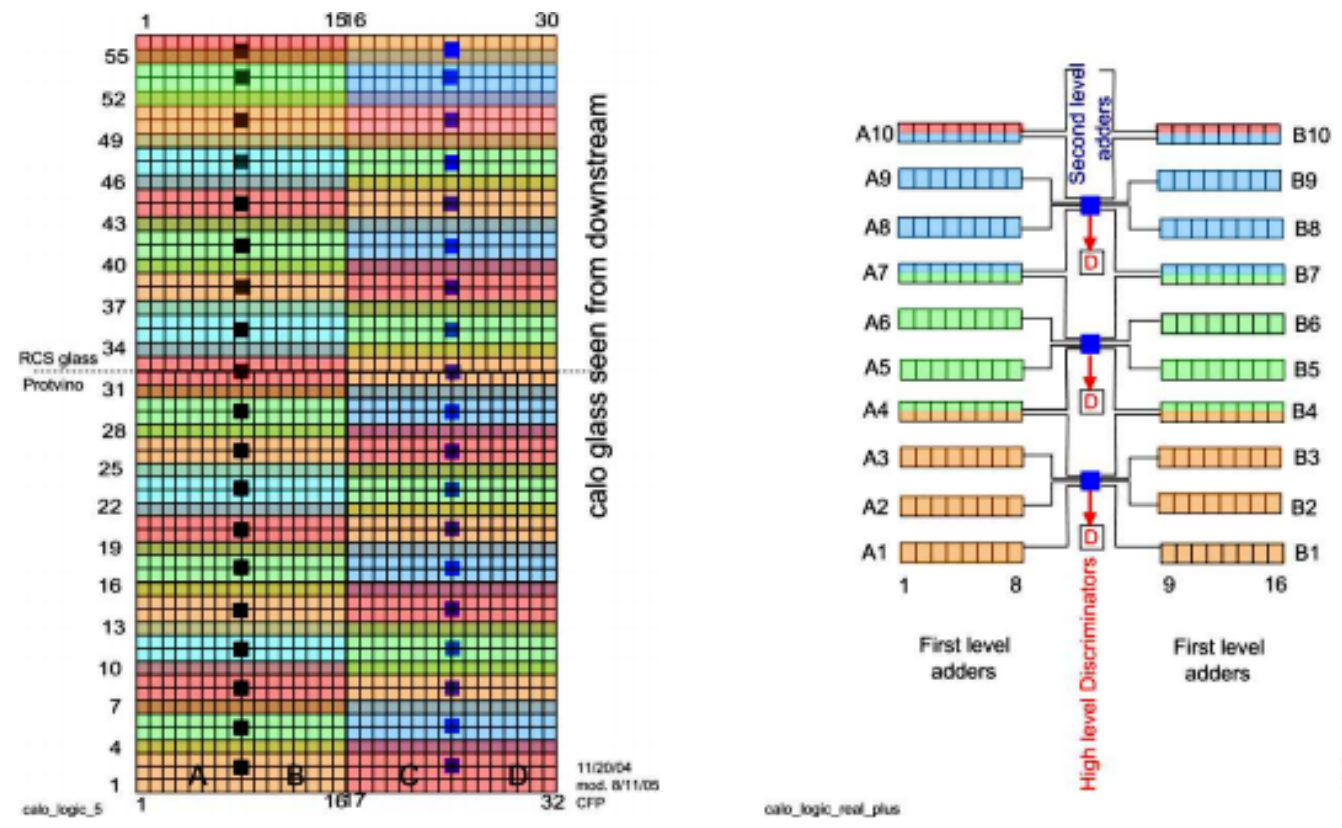

Figure 3.15

Rear View of Lead-Glass in BigCal (Left), Trigger Logic (Right)

\subsection{2 Čerenkov Detector}

The Čerenkov counter built by Temple University provided high efficiency (greater than $90 \%$ ) for electron detection while maintaining a pion rejection factor of at least 1000:1. With a low areal density, the Čerenkov detector minimizes the probability of $\delta$-rays from $\pi$ and $e$ scattering. It consisted of two stacks of four mirrors each that reflect light produced in nitrogen at atmospheric pressure and focuses it onto eight 3 inch diameter photomultipliers.

Čerenkov radiation is emitted when the speed of a speed of a particle, $v$ exceeds the speed of light in the medium it is traveling in with index of refraction $n: v>c / n$. 
The emission of Čerenkov radiation occurs at an angle given by:

$$
\theta=\cos ^{-1}\left(c_{n} / v\right)
$$

for a particle traveling at speed $v$. The electron detected will all be traveling close enough to the speed of light that the argument of $\cos ^{-1}$ is $1 / n$, and so the Čerenkov radiation is emitted almost parallel to the flight of the particle at about $\theta=1.4^{\circ}$.

Eight Mirrors were designed such that each mirror will reflect every ray traced from the target to the mirror's surface to a PMT dedicated to that mirror. The Čerenkov detector was made up of 8 PMTs. Half of the mirrors were toroidal and half of the mirrors were spherical.

Careful selection of the material based on its index of refraction allows identification of charged particles with speed above a given threshold. While electrons and pions of similar momentum or energy may be collected in the calorimeter, the more massive pions will not exceed the threshold speed, allowing rejection of the unwanted background.

Nitrogen $\left(N_{2}\right)$ gas was used as the choice of radiator gas in the design of the Čerenkov. At $20^{\circ} \mathrm{C}$, the index of refraction, $n$ of $N_{2}$ is approximately 1.000279 , yielding a $\beta$ threshold for Čerenkov light emission by pions of

$$
\beta_{\text {threshold }}=\frac{1}{n}=0.999721
$$

Also, the number of Čerenkov photons emitted per wavelength per unit of length travel for $N_{2}$ gas at $20^{\circ}$ assuming a constant index of refraction is given by:

$$
\frac{d N}{d \lambda}=\frac{2 \pi z^{2} \alpha}{\lambda^{2}}\left(1-\frac{1}{\beta^{2} n^{2}}\right)
$$


For $n=1.00279$ a conservative cutoff wavelength of $\lambda=200 \mathrm{~nm}$ and a radiation thickness of $125 \mathrm{~cm}$, we can expect the order of 20 photoelectrons after considering the photocathode sensitivity.

\subsubsection{Hodoscopes}

BETA had two tracking hodoscopes; namely, the lucite and forward tracker hodoscopes. These were contributed by North Carolina A\&T State University and Norfolk State University respectively.

\subsubsection{Lucite Hodoscope}

The Lucite Hodoscope was located between the Čerenkov and the BigCal calorimeter, at $253.6 \mathrm{~cm}$ from the target. While the distance between the Lucite and BigCal was 81.8 $\mathrm{cm}$. The purpose of the lucite hodoscope was: (a) to detect charged particles above the threshold (primarily electrons and pions) with high efficiency, (b) to assist in providing a high level of $\pi^{ \pm}$rejection (1000:1) for the case of electron trigger, (c) to provide useful position resolution at a reasonable cost, and (d) to be insensitive to the background particles coming from outside of the target chamber.

The index of refraction of the lucite is $n=1.49$ with a threshold velocity of Čerenkov radiation of $\beta_{\text {threshold }}=0.67$ inside. The bars of the lucite were wrapped in black paper without a reflecting layer ensuring that the propagation of light down the length of the bar to a photomultiplier tube (PMT) would be through total internal reflection (TIR) only. Since the critical angle inside the Lucite is $\theta_{T I R}=42.2^{\circ}$, this means only photons with an angle of incidence larger than $\theta_{T I R}=42.2^{\circ}$ would be detected. The PMT on both 
ends were shielded from the target magnetic field. The signals from the tubes were sent to discriminators then $\mathrm{TDCs}^{1}$ for recording.

\subsubsection{Forward (Front) Tracker}

The front tracker was the first element of the BETA detector package placed $55 \mathrm{~cm}$ away from the target cell. It constituted of three planes of $3 \times 3 \mathrm{~mm}^{2}$ Bicron-408 plastic scintillator bars positioned very close to the target. The main purpose was to provide tracking data on particles while they are still under the influence of the target's magnetic field. Combining this position data with the final positions caught in BigCal, the curve trajectory of the particle in the magnetic field should be perceptible, allowing the differentiation of positively and negatively charged particles. This would provide rejection of the positron background which diluted the yield of DIS electrons in BigCal. The tracker detector provided improved target position resolution in addition to its ability to reject non-target related backgrounds. An additional goal for the tracker is the partial ability to determine the sign of low momentum charged particles to discriminate positrons from electrons. This will allow the ability to measure positron asymmetry. Furthermore, this will enable the partial reduction of positron contamination of the electron sample and also reject low momentum $\pi^{+}$events. Figure 3.16 shows a picture of the front tracker [17].

\footnotetext{
${ }^{1}$ TDCs = Time-to-digital-converter
} 

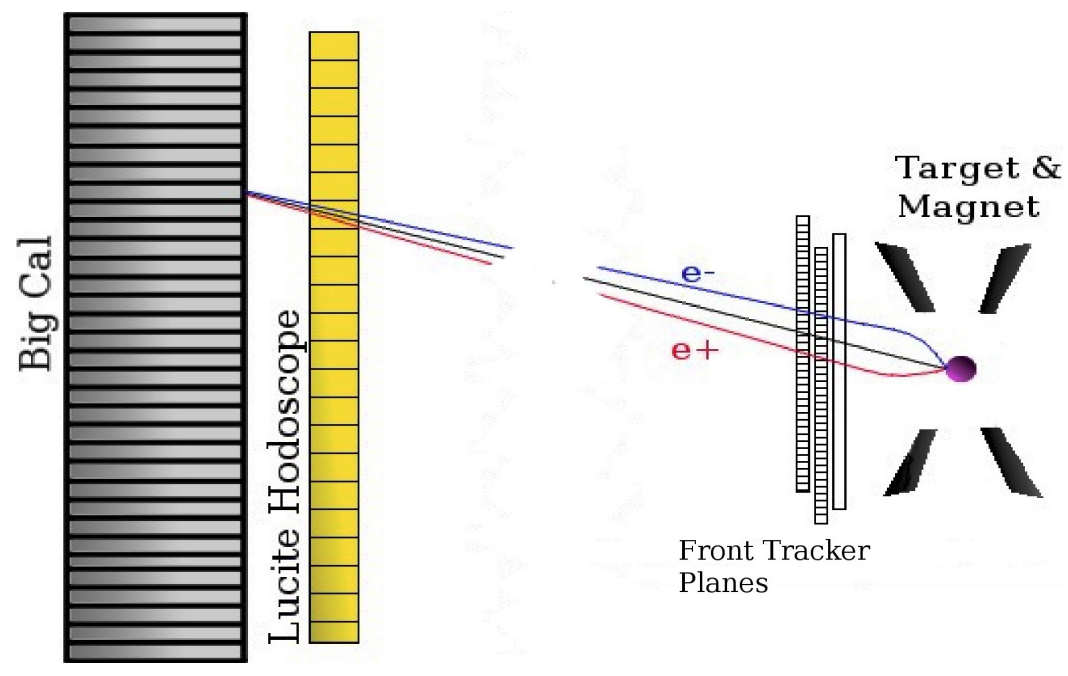

Figure 3.16

Picture of the front tracker 


\section{CHAPTER 4}

\section{DATA ANALYSIS}

This chapter talks about how the asymmetries and spin structure functions previously mentioned in chapter two are extracted and analyzed from the data collected during SANE.

The asymmetries measured from data collected from the BETA detector package are the following:

$$
A=\frac{1}{f P_{B} P_{T}} \frac{N_{C}^{+}-N_{C}^{-}}{N_{C}^{+}+N_{C}^{-}},
$$

where $N_{C}^{ \pm}=N^{+(-)} / C^{+(-)} / L^{+(-)} ; N^{+(-)}$is the number of counts with beam helicity positive (negative), $C^{+(-)}$is the incoming charge accumulated for each helicity and $L^{+(-)}$ is the livetime per helicity. The livetime will be discussed in section 4.3.2. Thus $N_{C}^{ \pm}$can be read as the corrected event counts for events generated by the indicated beam helicity. $P_{B}$ and $P_{T}$ correspond to the beam and target polarization respectively and are independent of the kinematic variables. They have fixed values assigned to them on a run by run basis. $f$ is known as the dilution factor and is different for each target load and is a function of the kinematics.

\subsection{Calibration}

In order to have the data in meaningful form, the various detectors in BETA needed to be calibrated. 


\subsubsection{BigCal Calorimeter}

The calorimeter was calibrated using a neural network (NN) (the NN will be discussed later). Calibration started at the level of the hardware. When energy is deposited by an incident particle into a lead-glass block of the calorimeter, a signal is generated by the photomultiplier tube and is digitized by an ADC ${ }^{1}$. This ADC signal is calibrated such that each ADC channel corresponds to roughly $1 \mathrm{MeV}$. The ADC signals with known energy are then analyzed. The absolute energy calibration of the $\mathrm{BigCal}$ is based on the reconstruction of the $\pi^{0}$ mass from events with two clusters of hits in the calorimeter produced by two photons.

Events for the calibration are based on: (a) minimum energy $E_{\gamma}>0.5 \mathrm{GeV}$, (b) distance between the clusters in the range $d_{\gamma \gamma} \in[20,90] \mathrm{cm}$, and (c) no signals in the Cerenkov for both clusters.

The definition of a cluster was set as a $5 \times 5$ array of blocks around the maximum energy block. The raw energy and coordinates of the clusters, calculated from the sum of the block energies and the energy weighted $x$ and $y$ cluster centroids, were corrected using the output of an artificial neural network (see section 4.1.2). The final physics angles were arrived at using the functions: $\Theta=f(X, Y, E)$ and $\Phi=g(X, Y, E)$ obtained from a GEANT Monte Carlo simulation by fitting the detected coordinates and energy of the particle to the corresponding angles of the generated event.

Meanwhile, the invariant square mass of the two decay photons is determined by:

$$
M_{\gamma \gamma}^{2}=2 E_{1} E_{2}\left(1-\cos \theta_{\gamma_{1} \gamma_{2}}\right)
$$

\footnotetext{
${ }^{1} \mathrm{ADC}=$ Analogue-to-digital-converter
} 
$M_{\gamma \gamma}^{2}$ is clearly directly proportional to the energies $E_{1}$ and $E_{2}$ of the photons. In this process, the essential assumption in the corrections to the raw energy is that the difference between the physical mass of the neutral pion $\left(m_{\pi^{0}}\right)$ and the mass reconstructed from the photon pair mass comes from just one of the cluster energies, thus:

$$
M_{\gamma \gamma}^{2}(\text { recon })=2 E_{1} E_{2}(\text { recon })\left(1-\cos \theta_{\gamma_{1} \gamma_{2}}\right)
$$

or

$$
M_{\gamma \gamma}^{2}=2 E_{1}(\text { recon }) E_{2}\left(1-\cos \theta_{\gamma_{1} \gamma_{2}}\right) .
$$

Since it is assumed that the difference in mass comes from just one of the cluster energies. We set the correction factors $C_{n}$ where:

$$
C_{n}=\frac{M_{\gamma \gamma}^{2}(\text { recon })}{m_{\pi^{0}}} .
$$

These correction factors are applied to blocks containing at least $20 \%$ of the cluster energy.

Upon the implementation of neural network position reconstruction, the angle $\theta_{\gamma_{1} \gamma_{2}}$ sustained between the two photons changes slightly after calibration. This is corrected by performing the calibration procedure over five hundred times in order to reach full convergence. The pion mass resolution obtained by this procedure is illustrated in Figure 4.1 [36]. Furthermore, this resolution is directly proportional to the energy resolution of the clusters.

\subsection{Artificial Neural Network}

Artificial neural networks are systems of interconnected neurons which can compute values from inputs. A neuron is a complicated function with inputs chosen based on what 


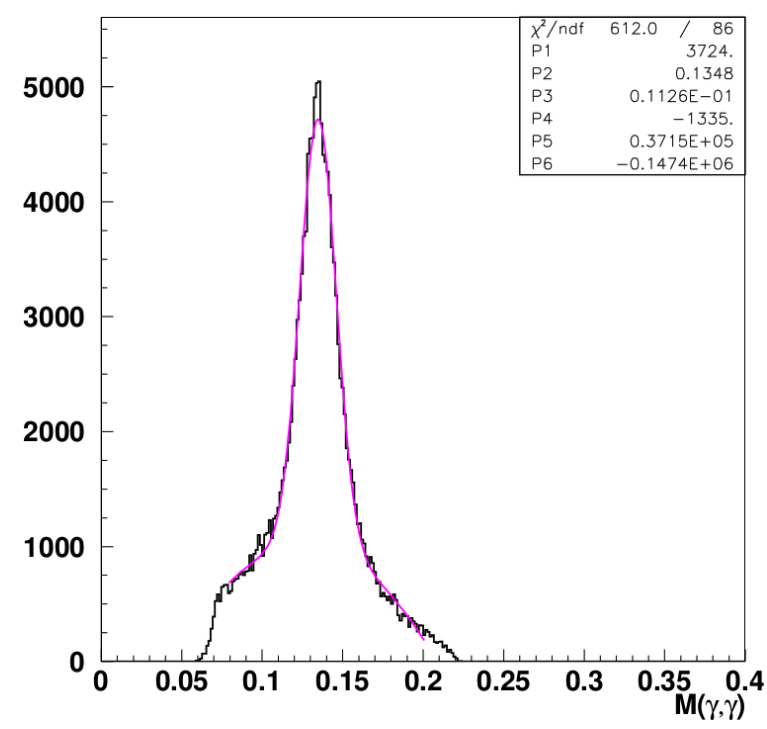

Figure 4.1

Reconstructed $\pi^{0}$ Mass. $P_{2}=$ Gaussian Fit and $P_{3}=\sigma$

the desired output is expected to depend on. The neural network was trained with output based on a GEANT simulation of the experiment that includes the polarized target magnetic field, the beam's slow raster $(\leq 1.2 \mathrm{~cm}$ radius $)$ and BETA. The input neurons to the $\mathrm{NN}$ came from the energies of 25 blocks surrounding the maximum energy block and the $X$ and $Y$ coordinates of the maximum energy block. The NN consists of one hidden layer with ten neurons. Output neurons are the cluster energy and coordinate corrections. Figure 4.2 shows a schematic diagram of the artificial neural network and this was based on the ROOT standard package (Multilayer Perceptron) [36]. The neuron training function was chosen to be Gaussian and the learning method to be KBFGS (TMultiLayerPerceptron) [67]. 


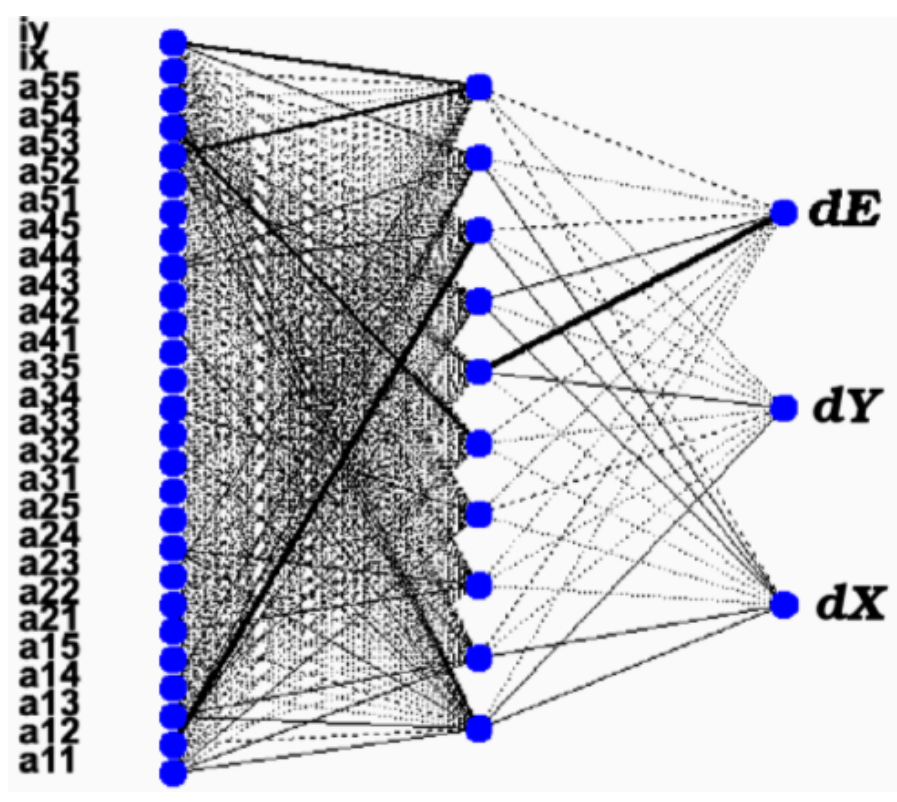

Figure 4.2

Neural Network schematics

More than twenty million events of electrons and photons were generated and used to train the NN with over 1000 epochs involved in the training procedure. An epoch is a measure of the number of times all of the training functions are used once to update the weights. The time taken to complete such a training procedure is about a month on a computer equipped with dual $2.7 \mathrm{GHz}$ quad-core Xeon CPU.

The generated photons were important for the different cluster energy distributions of the photon showers, which start one radiation length deeper in the glass than the electron showers. In addition, the NN was used to obtain the angles at the target by training the NN to fit the reconstructed polar and azimuthal GEANT-simulated angles to the corresponding generated quantities. The resulting fits are then applied to the data's reconstruction. $\sigma_{\Theta}=$ $0.4^{\circ}$ and $\sigma_{\Phi}=0.8^{\circ}$ were obtained as the resulting angular resolutions at the target. 
In Figure 4.3 the difference using $\mathrm{NN}$ and the conventional method is shown. The difference between the GEANT generated and reconstructed positions and energy using the conventional moments method (solid blue line) and with NN (dashed red line). As can be seen in panels a) and b) there is a dramatic position reconstruction improvement in both the $X$ and the $Y$ directions amounting to a resolutions improvement from $4 \mathrm{~cm}$ to $1 \mathrm{~cm}$. A detailed discussion of the NN can be found [67].
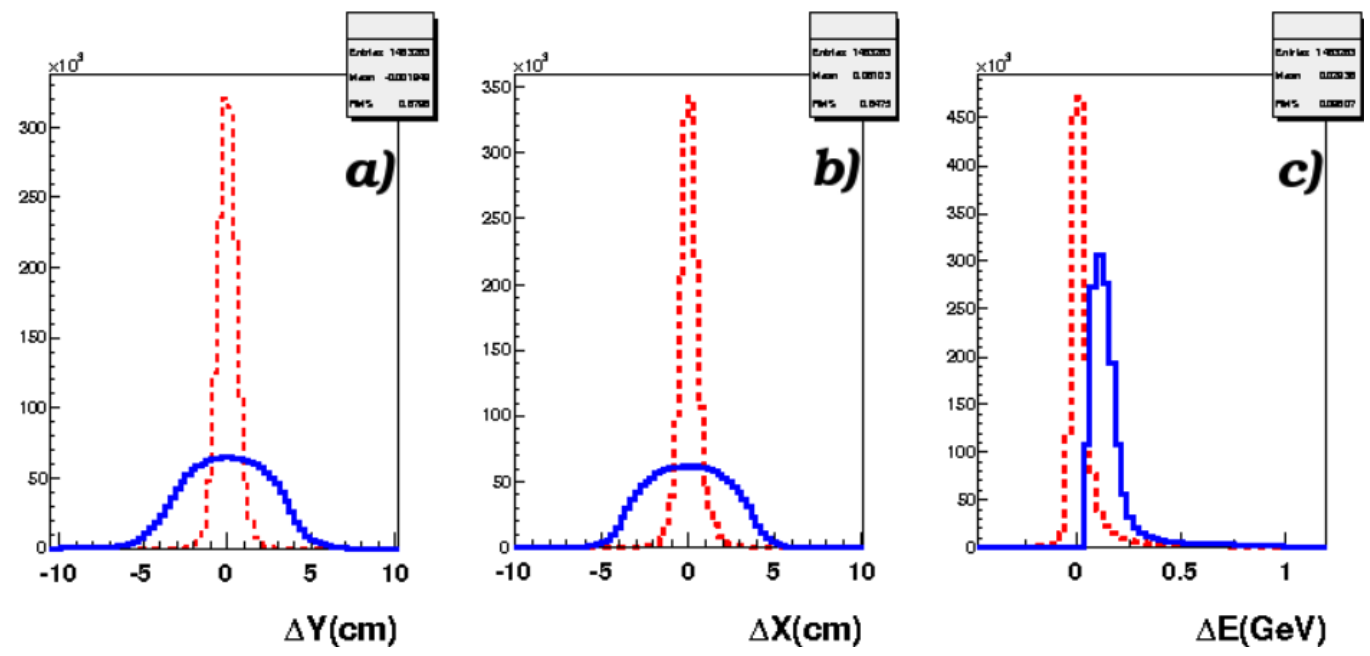

Figure 4.3

Difference between generated and reconstructed $Y, X$, and $E$

\subsection{1 Čerenkov}

In the analysis of the Čerenkov, recorded $\mathrm{TDC}^{2}$ and $\mathrm{ADC}^{3}$ values of the electron event were required. The TDC value for an event, which was triggered by a threshold on a photomultiplier analog signal was sufficient to tag an event as charged or not. The ADC

\footnotetext{
${ }^{2}$ TDCs = Time-to-digital-converter

${ }^{3} \mathrm{ADCs}=$ Analogue-to-digital-converter
} 
spectra show two peaks, for single and double tracks, the double track peak (Figure 4.4) was used to cut pairs from the tracker background as explained in section 5.2.1. A timewalk correction was also applied in the calibration of the Cerenkov. A time-walk is a shift in the trigger time based on the peak height of an ADC signal where a discriminator triggers on a threshold of the ADC signal from a photomultiplier tube. The distribution of the Čerenkov TDC versus ADC was plotted to correct for this shift (see Figure 4.5).

\subsection{Event Reconstruction and Selection}

An important element of the analysis is the event reconstruction phase. Once the various detectors are calibrated, we obtain a set of events, each of which consists of ADC and TDC values from the different detectors. These detector signals need to be reconstructed into the path of an electron of energy and trajectory which must be determined via the three quantities of interest for each event namely: the final electron energy $E^{\prime}$ and the electron scattering angles $\phi$ and $\theta$.

Knowing the $x$ and $y$ coordinate position of a cluster in BigCal by way of the neural network, it becomes straightforward to arrive at the scattering angles $\phi_{B}$ and $\theta_{B}$ in BigCal coordinates which are related to the physical scattering angles $\phi$ and $\theta$ in the following manner:

$$
\begin{aligned}
\cos \theta & =\cos \left(\theta_{B E T A}-\theta_{B}\right) \cos \phi_{B} \\
\tan \phi & =\frac{\tan \phi_{B}}{\sin \left(\theta_{B E T A}-\theta_{B}\right)}
\end{aligned}
$$

where $\theta_{B E T A}=40^{\circ}=$ the BigCal central angle from the beam. Figure 4.6 depicts BETA and the physics angles [16]. 


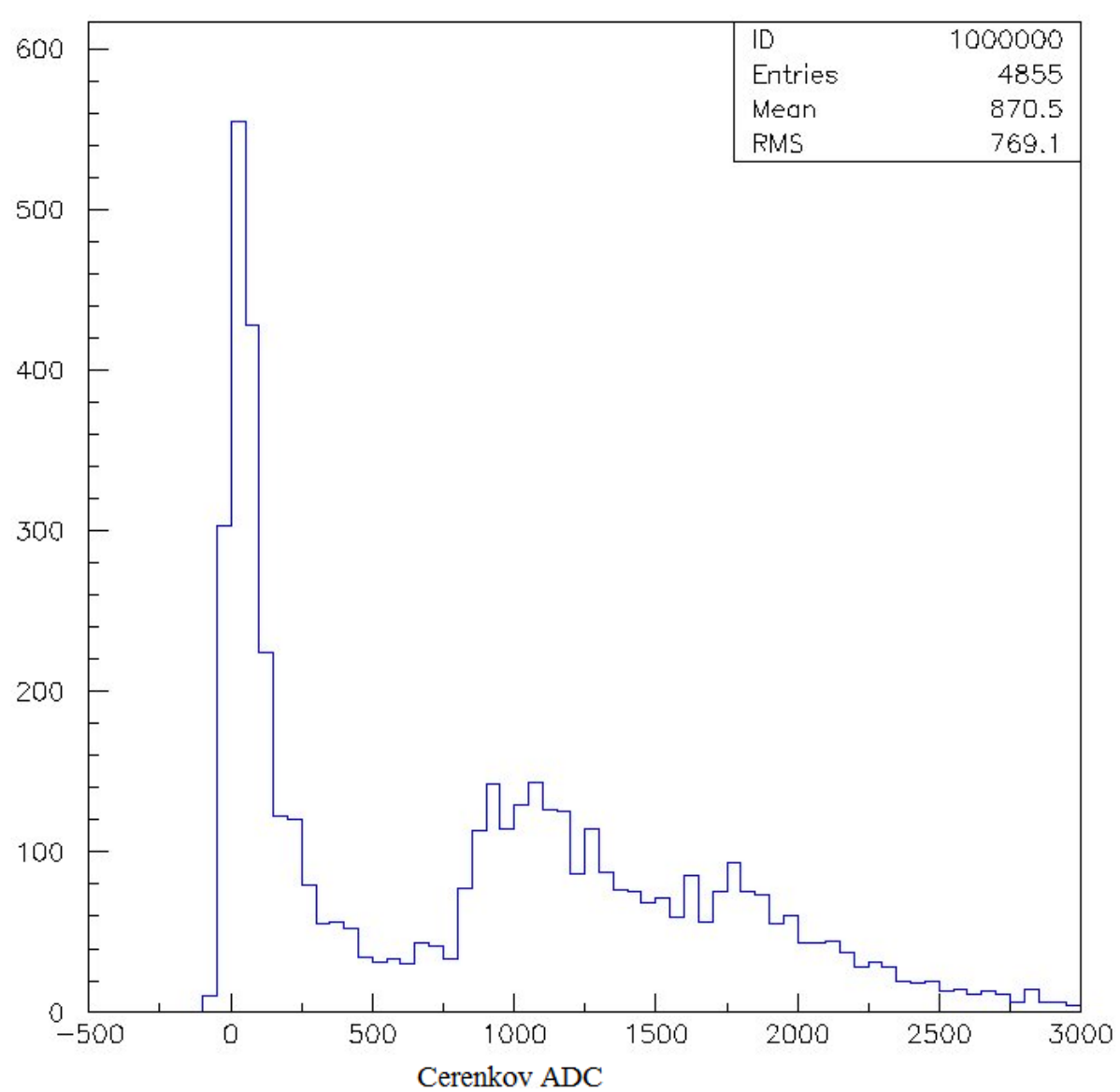

Figure 4.4

Čerenkov ADC Showing Two Peaks 

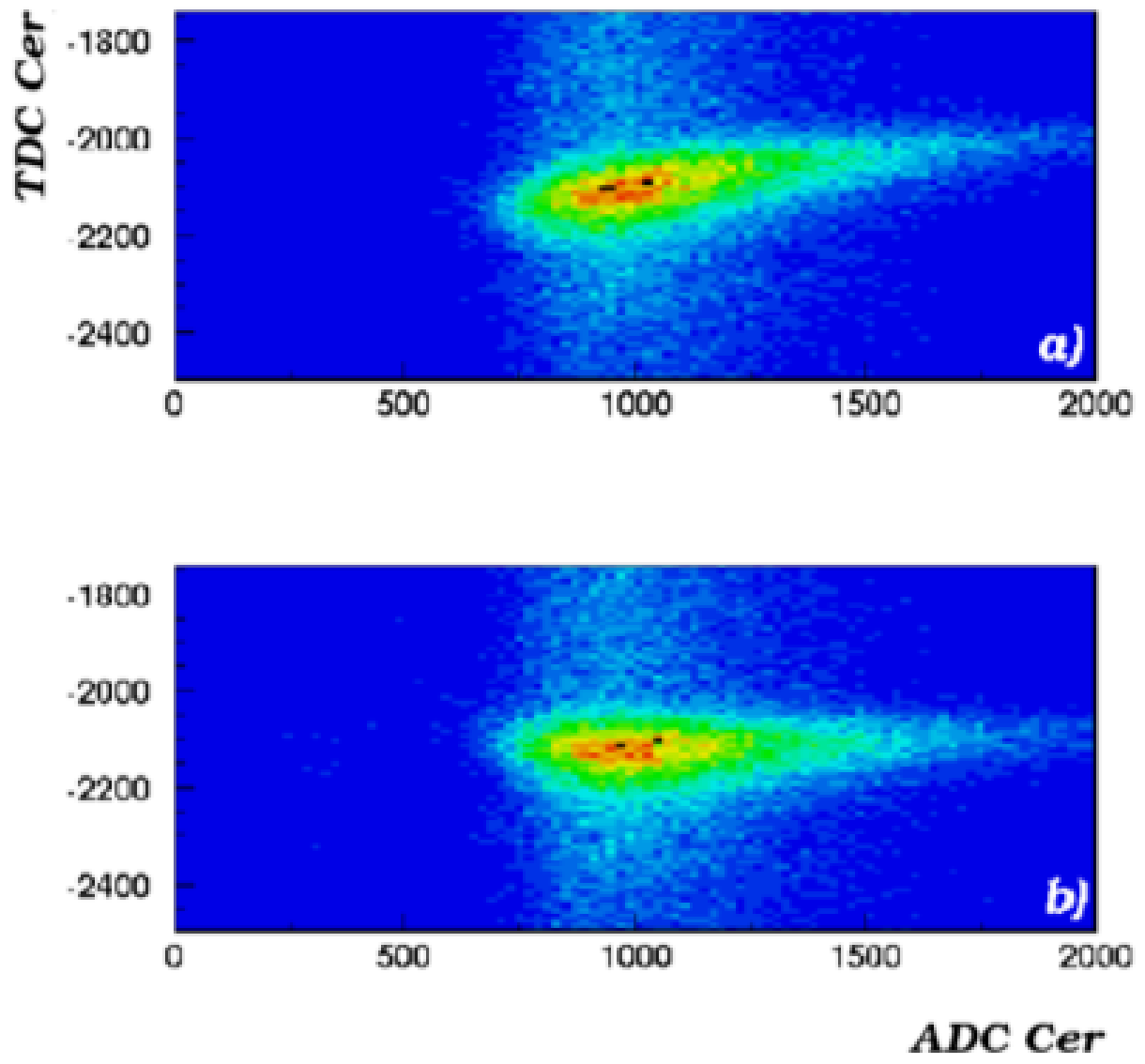

Figure 4.5

Čerenkov TDC versus ADC values 


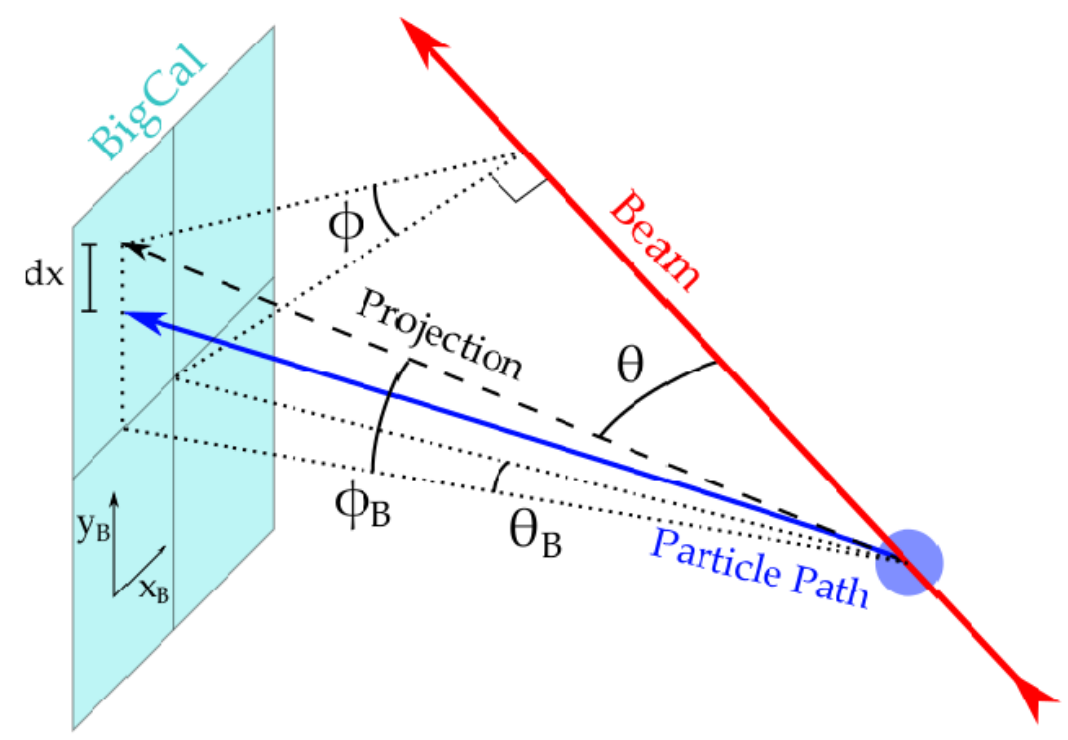

Figure 4.6

Diagram of BETA with Physics Angles

\subsubsection{Event Criteria}

It is important to select only the electron events of interest during the scattering process.

In order to accomplish this, we set several criteria to the events which will be included in the helicity count $N^{+}$and $N^{-}$. These criteria are listed as follows:

1. Trigger Type: Only trigger for events with a hit in both the calorimeter and the Čerenkov are considered.

2. Single Cluster: Only events with a single cluster on BigCal were included.

3. Čerenkov Hash: This cut takes care of events that occur in the calorimeter. This cut is greater than zero in the case of a good Čerenkov hit at the perfect time frame, and this hit matched a geometrical cut with the calorimeter.

4. Energy Cluster: The energy cluster cut was set at $500 \mathrm{MeV}$, this excluded charged hadron events which are unlikely to be found about $500 \mathrm{MeV}$.

5. Cluster Position: This cut helps to avoid events arriving on the edges of the calorimeter which are difficult to calibrate. 
6. Beam Current: This cut makes sure only events occurring when the beam current is over $60 \mathrm{nA}$ are considered.

With the event criteria, and selection in place, the data of the experiment were taken such that any run which is suspected to be undesirable is rejected. Undesirable runs were runs with either end-of-run errors, unacceptable low livetimes, i.e., the deadtime (lost time) of the data acquisition system (see section 4.3.2), asymmetries which were statistical out of bound or those labeled by the operators as bad. Each experimental run lasted for about one hour. Of all the experimental runs, about $315 \mathrm{NH}_{3}$ good run were selected, and compiled. A table of the selected $\mathrm{NH}_{3}$ runs with the energy and angle orientation is shown in Table 4.1.

Table 4.1

Good Runs with the Energy and Target Field Angle

\begin{tabular}{cccc}
\hline Energy & Angle Orientation & Run Range & Number of Good Runs in Range \\
\hline 5.9 & $80^{\circ}$ & $72417-72799$ & 222 \\
4.7 & $80^{\circ}$ & $72824-72892$ & 33 \\
5.9 & $180^{\circ}$ & $72915-72959$ & 27 \\
4.7 & $180^{\circ}$ & $72984-73037$ & 30 \\
\hline
\end{tabular}

\subsection{Asymmetries and Structure Functions Analysis}

Equation (4.1) shows how the asymmetries are measured from the data collected during the experiment. It can be rewritten in the form:

$$
A_{\text {physics }}=\frac{1}{f P_{B} P_{T}} A_{\text {measured }}
$$


Where $A_{\text {measured }}$ is:

$$
A_{\text {measured }}=\frac{N_{+}-N_{-}}{N_{+}+N_{-}}
$$

$N_{+}$and $N_{-}$are positive and negative electron yields produced for each kinematic bin.

However, in order to have clean physics asymmetry result, the measured electron must go through several corrections such as: charger normalization, livetime correction, dilution factor, nitrogen, radiation and pair-symmetric background corrections. All the above mentioned corrections will be discussed briefly in this section except for the pair symmetric background that will be discussed in greater detail in chapter 5 .

\subsubsection{Charge Normalization}

Charge normalization helps to prevent false asymmetry and is done by taking into account the incoming charge accumulated during the positive and negative helicity events. Hence $A_{\text {measured }}$ becomes

$$
A_{\text {charge normalization }}=\frac{\frac{N_{+}}{C_{+}}-\frac{N_{-}}{C_{-}}}{\frac{N_{+}}{C_{+}}+\frac{N_{-}}{C_{-}}}
$$

where $C_{+}$and $C_{-}$are the charge accumulated for positive and negative helicities. $C_{+}$and $C_{-}$were taken from scaler data archive for SANE runs.

\subsubsection{Livetime Correction}

The livetime correction to the asymmetry takes into accounts the deadtime (lost time) of the data acquisition (DAQ). The livetime is the DAQ recorded positive or negative helicity triggers divided by input triggers of that helicity recorded in scalers. Unfortunately, the 
positive helicity input trigger scaler information was lost. So the livetime (LT) for each run is determine using the negative helicity trigger as:

$$
L T=\frac{R_{T}}{R_{I}}
$$

where $R_{T}$ is the rate of negative helicity triggers and $R_{I}$ is the input trigger rate.

The total positive helicity triggers was estimated by assuming the correlation of the recorded positive helicity triggers to the total positive helicity triggers is the same as that of the negative helicity triggers to their total. By fitting the linear correlation of the negative helicity total scalers and recorded scalers that we have, we can calculate the total positive triggers using the recorded positive triggers.

\subsubsection{Dilution Factor and Packing Fractions}

The dilution factor is the ratio of yields from the polarized protons to yields from all the materials in the target sample. The dilution factor $f\left(Q^{2}, W\right)$ is used to calculate the measured asymmetries which are defined in terms of scattering on the protons. For ${ }^{14} \mathrm{NH}_{3}$, the dilution factor has the form:

$$
f=\frac{N_{1} \sigma_{1}}{N_{14} \sigma_{14}+N_{1} \sigma_{1}+\sum N_{A} \sigma_{A}}
$$

where $N_{A}$ are the number of scattering nuclei of mass number $A$ per unit area in the target, and $\sigma_{A}$ are the radiated, polarized $e-$ nucleus $A$ cross sections and are functions of invariant mass $W$. A dilution factor is necessary for each and every target load used during the running of SANE.

The origin of the dilution factor can be understood by looking closely at the definition of the measured asymmetry being the ratio of the difference over the sum of helicity de- 
pendent counts observed in the detector. Consider the formula used for calculating the raw asymmetry, $A_{\text {raw }}$ given by

$$
A_{\text {raw }}=\frac{N^{+}-N^{-}}{N^{+}+N^{-}}
$$

$A_{\text {raw }}$ can be rewritten as:

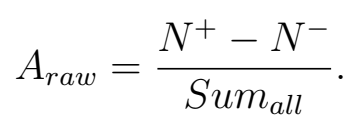

From equation (4.13), it can be seen that the numerator is composed solely of polarized counts (that is $N_{\text {proton }}^{+}-N_{\text {proton }}^{-}$), this is due mainly because the counts for the unpolarized materials cancel in the difference in the numerator. In the denominator, there is no such cancellation, rather it contains both the polarized and unpolarized counts.

For the case of the asymmetry of the proton only,

$$
A_{\text {proton }}=\frac{N_{\text {proton }}^{+}-N_{\text {proton }}^{-}}{\text {Sum }_{\text {proton }}}
$$

therefore $A_{\text {raw }}$ can be expressed as:

$$
A_{\text {raw }}=\frac{N_{\text {proton }}^{+}-N_{\text {proton }}^{-}}{\text {Sum }_{\text {proton }}} * \frac{\text { Sum }_{\text {proton }}}{\text { Sum }_{\text {all }}}=A_{\text {proton }} * f .
$$

Therefore

$$
A_{\text {proton }}=\frac{1}{f} \cdot A_{\text {raw }} .
$$

Figure 4.7 shows the dilution factor for one of the $\mathrm{NH}_{3}$ target load as a function of $\mathrm{W}$ [83].

However, the dilution factors depend on the packing fraction, which is mainly the fractional volume of the target cup filled with ammonia. It is a percentage and is independent 


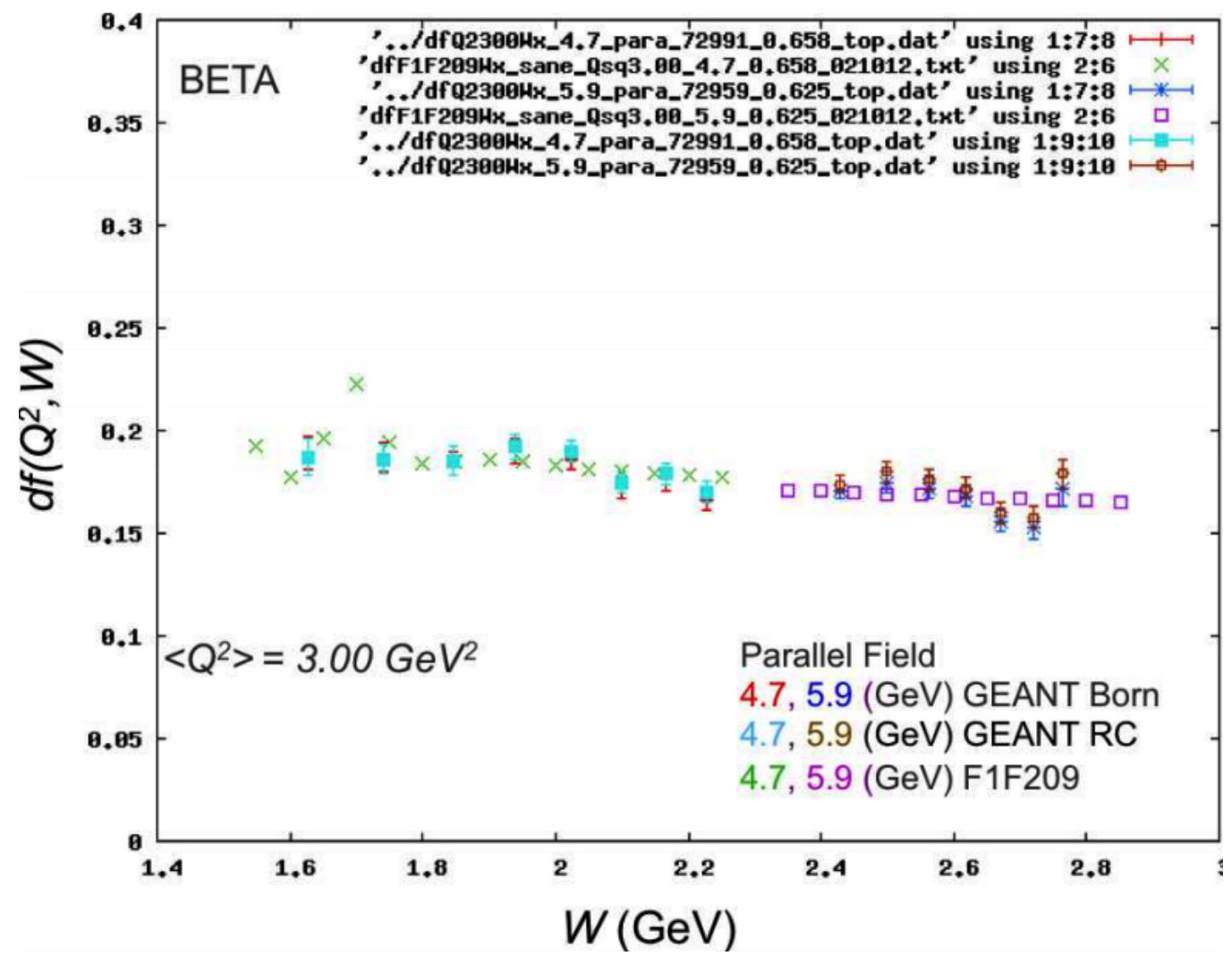

Figure 4.7

Dilution factor for one of the $\mathrm{NH}_{3}$ target loads as a function of $W(\mathrm{GeV})$ 
of kinematics. HMS data taken on ${ }^{14} \mathrm{NH}_{3}$ and on carbon disk targets of known thickness were used to calculate the packing fractions. The need to calculate the packing fraction can not be over emphasized, as we endeavor to fill the target cup completely, differences in the load amount, and the size and shape of the target beads change the packing fraction from load to load. By comparing the yields from each target load to those using a carbon disc target of known thickness, the packing fraction was estimated using the linear relation:

$$
\text { Yield }=m \times P_{f}+b
$$

where the slope $m$ and the intercept $b$ depend on the beam current, acceptance, partial densities and cross sections. With the linear form, the packing fraction of a given load can now be calculated by interpolating between two reference points on the line. These two points can come from a Monte Carlo simulation which accurately represented the acceptance of the detectors and the cross sections of the target materials involved. An important consideration is the production of a scaling factor to bring the Monte Carlo yields into agreement with the carbon data. The packing fraction is then a simple linear interpolation between the Monte Carlo yields with a target of packing fraction $P_{f 1}$ and another of packing fraction $P_{f 2}$. The $\mathrm{NH}_{3}$ data was simulated with two input packing fractions $(50 \%$ and $60 \%)$ to obtain two reference points, from which the slope $m$ and the intercept $b$ of the line were determined

$$
\begin{aligned}
m & =\frac{\text { Yield }_{P_{f}=0.5}-\text { Yield }_{P_{f}=0.6}}{0.5-0.6} \\
b & =\text { Yield }-m \times P_{f} .
\end{aligned}
$$


Figure 4.8 shows the interpolation between simulated yields of packing fraction 0.5 and 0.6 to obtain packing fraction from the experimental yield [16].

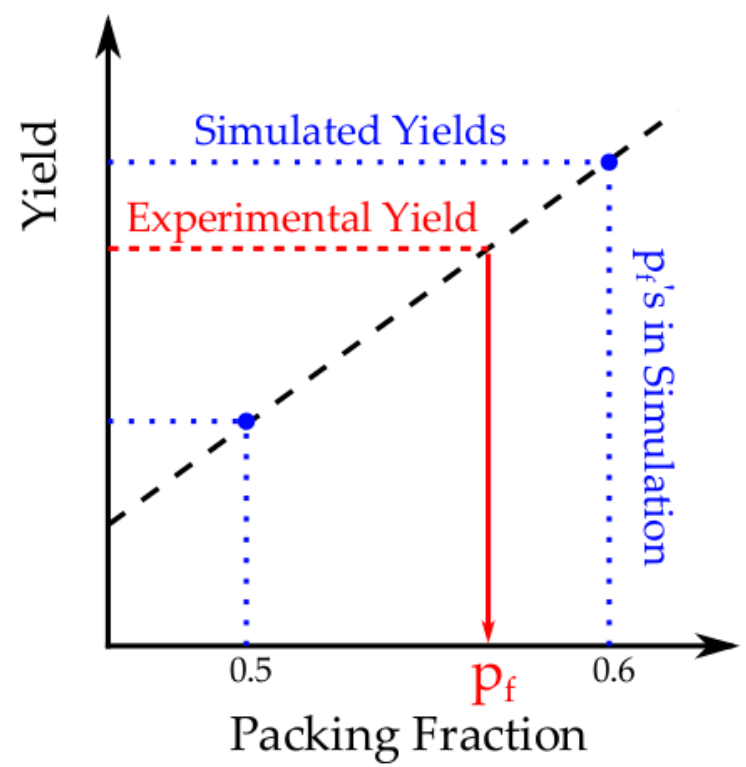

Figure 4.8

Method Used in Estimating the Packing Fraction in SANE

The packing fractions determined averaged $\sim 59.60 \%$, ranging $\sim 3.30 \%$ (absolute) about this mean [74]. The error on the $P_{f}$ was about $8 \%$ relative. This error propagates to the dilution factor but is suppressed by a factor of about two.

With the ammonia's calculated packing fraction the dilution factor $f\left(Q^{2}, W\right)$ is computed as:

$$
f\left(Q^{2}, W\right)=\frac{N_{1}\left(P_{f}\right) \sigma_{1}\left(Q^{2}, W\right)}{\sum_{A=1,2, \ldots} N_{A}\left(P_{f}\right) \sigma_{A}\left(Q^{2}, W\right)}
$$

Where $N_{A}\left(P_{f}\right)$ are the numbers of scattering nuclei of mass number $A$, density $\rho_{A}$, and atomic or molecular weight $M_{A}$ that occupy a length $z_{A}=3 \mathrm{~cm} \times p f$ in the target 
cup assuming constant area along the horizontal cylindrical cell. Also $N_{A}$ are computed in terms of Avogadro's number and is given by:

$$
N_{A}\left(P_{f}\right)=\frac{N_{0} \rho_{A} z_{A}}{M_{A}}
$$

Equation (4.20) has units of $\mathrm{cm}^{-2}$.

\subsubsection{Radiative Corrections}

Radiative corrections carried out in SANE involve both internal and external radiative corrections and depended upon the thickness of material encountered by the incoming and scattered electron [68].

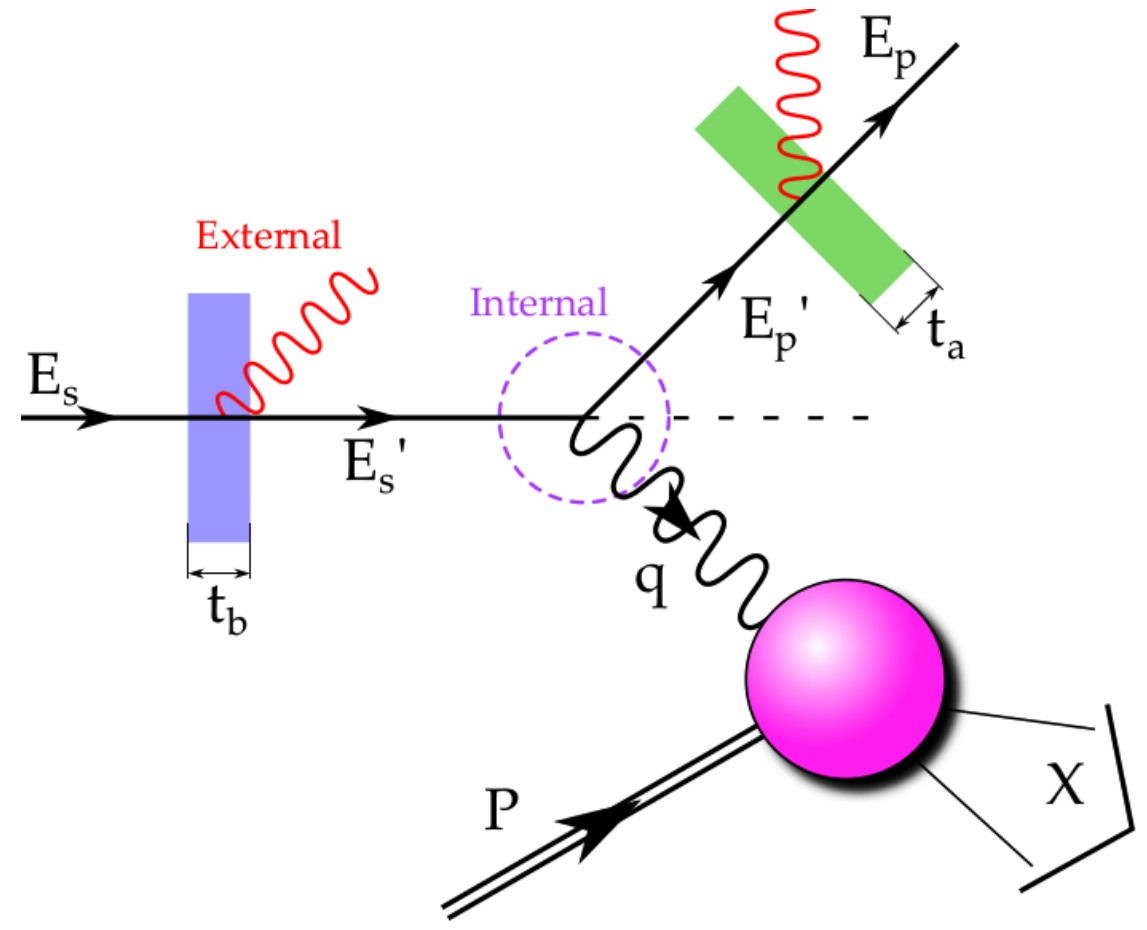

Figure 4.9

Radiation corrections mechanism 
Since SANE was an inclusive electron scattering on a polarized proton target, the observed asymmetries needed to be corrected for losses as a result of external and internal radiative processes. See Figure 4.9 [16, 69].

External corrections are essential to address the processes of bremsstrahlung and ionization in all material traversed by the electron beam before and after the scattering process of interest occurred. These materials which contribute to the radiation length include; aluminum beam windows, nose, helium, ammonia, etc or an electron may even radiate a photon before and after the scattering of interest at a probability related to the radiation length. On the other hand, internal corrections involve vacuum polarization, vertex corrections and internal bremsstrahlung. Due to external radiative processes, the incident beam energy $E_{s}$ and reconstructed final electron energy $E_{p}$ are changed to the $E_{s}^{\prime}$ and $E_{p}^{\prime}$ to the energies of the $e-p$ interaction. Thus the radiative effects act on the data in such a way as to shift the true kinematics at which the interaction takes place away from the measured kinematics (see Figure 4.9). Table 4.2 also shows a list of the radiation lengths.

Contribution to the radiative correction is broken down into two aspects: contribution from the elastic radiative corrections and contribution from inelastic radiative corrections (the inelastic contribution is small though). Most of the work done on the elastic radiative corrections was done by one of SANE collaborators, J. Maxwell [69]. A detailed recipe of the radiative corrections is covered in the following references [72]. 
Table 4.2

Material thickness that contributed to radiative processes

\begin{tabular}{lccc}
\hline & Material & Thickness $\left(\mathrm{mg} / \mathrm{cm}^{2}\right)$ & Rad. Length $(\%)$ \\
\hline Target Ammonia & ${ }^{4} \mathrm{NH}_{3}$ & 1561 & 3.82 \\
Target Helium & $\mathrm{LHe}$ & 174 & 0.18 \\
NMR Coil & $\mathrm{Cu}$ & 13 & 0.10 \\
Target Cell Lid & $\mathrm{Al}$ & 10 & 0.04 \\
Refrigerator Window & $\mathrm{Al}$ & 27 & 0.12 \\
4K Radiation Shield & $\mathrm{Al}$ & 7 & 0.03 \\
Nitrogen Radiation Shield & $\mathrm{Al}$ & 10 & 0.04 \\
Vacuum Chamber Entrance & $\mathrm{Be}$ & 94 & 0.14 \\
Vacuum Chamber Exit & $\mathrm{Al}$ & 139 & 0.58 \\
\hline
\end{tabular}

\subsection{Structure Function from Measurable Asymmetries and Cross-Section}

In this subsection we discuss how to get from the measured asymmetries $A_{80^{\circ}}$, and $A_{180^{\circ}}$ to the structure functions $g_{1}$, and $g_{2}$ and the spin asymmetries $A_{1}$, and $A_{2}$.

For the process of inclusive electron scattering off a nucleon target, the difference of cross-section is given by

$$
\begin{gathered}
\Delta \sigma_{\|}=\frac{-4 \alpha^{2} E^{\prime}}{Q^{2} E}\left[\left(E+E^{\prime}\right) M G_{1}-Q^{2} G_{2}\right] \\
\Delta \sigma_{\perp}=\frac{-4 \alpha^{2} E^{\prime 2}}{Q^{2} E} \sin \theta \cos \phi\left(M G_{1}+2 E G_{2}\right)
\end{gathered}
$$

It should be noted that $\Delta \sigma_{\|}$and $\Delta \sigma_{\perp}$ are the proper parallel and perpendicular crosssection. However in SANE where the coordinate system is such that the $z$-axis points along the beamline towards the beam dump, the $x$-axis is horizontal pointing towards the BigCal side of the beamline, and the $y$-axis points up, we need the asymmetries for the spin associated with target polarization anti parallel to the beam and for the target polarization aligned $10^{\circ}$ off from the $x$-axis in the $x-z$ plane. 
This was accounted for by making use of the fact that by doing a general cross-section difference without specifying the angle of the target spin vector, but assuming it is in the $x-z$ plane one obtains:

$$
\Delta \sigma=\Delta \sigma_{\|} \cos \alpha+\Delta \sigma_{\perp} \sin \alpha
$$

Furthermore, with respect to the unpolarized cross-section $\left(\sigma^{u n p}\right)$ and the unpolarized structure functions, $W_{1}\left(M \nu, Q^{2}\right)$ and $W_{2}\left(M \nu, Q^{2}\right)$ we have:

$$
\frac{d^{2} \sigma^{u n p}}{d \Omega d E^{\prime}}=\frac{4 \alpha^{2} E^{\prime 2}}{q^{4}}\left[2 W_{1} \sin ^{2} \frac{\theta}{2}+W_{2} \cos ^{2} \frac{\theta}{2}\right]
$$

Moreover, $W_{1}$ and $W_{2}$ can be expressed with another structure function $R$ (where $R$ is the ratio of the longitudinal to the transverse Compton cross-section) such that:

$$
\frac{W_{2}}{W_{1}}=\frac{1+R}{1+\frac{\nu^{2}}{Q^{2}}}
$$

Hence:

$$
\frac{d^{2} \sigma^{u n p}}{d \Omega d E^{\prime}}=\frac{2 \alpha^{2} E^{\prime}}{Q^{2} E} W_{1}\left[1+\frac{1+R}{2\left(1+\frac{\nu^{2}}{Q^{2}} \tan ^{2} \frac{\theta}{2}\right)}\right]
$$

We can get a more compact expression by using the following substitutions:

$$
\begin{aligned}
\epsilon & =\frac{1}{1+2\left(1+\frac{\nu^{2}}{Q^{2}}\right) \tan ^{2} \frac{\theta}{2}} \\
D^{\prime} & =\frac{1-\epsilon}{1+\epsilon R}
\end{aligned}
$$

resulting to

$$
\sigma^{u n p}=\frac{d^{2} \sigma^{u n p}}{d \Omega d E^{\prime}}=\frac{2 \alpha^{2} E^{\prime}}{Q^{2} E} \frac{W_{1}}{D^{\prime}}
$$

Therefore the asymmetries can be written as:

$$
A=\frac{\Delta \sigma}{2 \sigma^{u n p}}
$$




\subsection{Extraction of Asymmetries and Structure Functions}

The spin asymmetries $A_{1}$ and $A_{2}$ are extracted via asymmetries measurements with longitudinal and transverse polarizations. However, in SANE we measured anti-parallel $\left(180^{\circ}\right)$ and near-perpendicular $\left(80^{\circ}\right)$ asymmetries, hence careful determination of axis angle are necessary.

In the case of SANE, the asymmetry associated with the near-perpendicular configuration is:

$$
\begin{aligned}
A_{80^{\circ}} & =\frac{-D^{\prime}}{W_{1}}\left\{\left[\left(E+E^{\prime} \cos \theta\right) \cos 80^{\circ}+E^{\prime} \sin \theta \cos \phi \sin 80^{\circ}\right] M G_{1}\right. \\
& \left.+\left(2 E E^{\prime} \sin \theta \cos \phi \sin 80^{\circ}-Q^{2} \cos 80^{\circ}\right) G_{2}\right\},
\end{aligned}
$$

while the asymmetry associated with the anti-parallel configuration is:

$$
A_{180^{\circ}}=\frac{D^{\prime}}{W_{1}}\left[\left(E+E^{\prime} \cos \theta\right) M G_{1}-Q^{2} G_{2}\right] .
$$

Since $A_{180^{\circ}}$ and $A_{80^{\circ}}$ form a basis, we can find linear combinations of them that produce the structure functions. By solving for $G_{1}$ and $G_{2}$, the solutions are:

$$
\begin{aligned}
\frac{M G_{1}}{W_{1}} & =-\frac{A_{1} 80^{\circ}\left(Q^{2} \cos 80^{\circ}-2 E E^{\prime} \sin \theta \cos \phi \sin 80^{\circ}\right)+Q^{2} A_{8} 0^{\circ}}{D^{\prime} E^{\prime} \sin \theta \cos \phi \sin 80^{\circ}\left[2 E\left(E+E^{\prime} \cos \theta\right)+Q^{2}\right]} \\
\frac{G_{2}}{W_{1}} & =-\frac{\left[\left(E+E^{\prime} \cos \theta\right) \cos 80^{\circ}+E^{\prime} \sin \theta \cos \phi \sin 80^{\circ}\right] A_{180^{\circ}}+\left(E+E^{\prime} \cos \theta\right) A_{8} 0^{\circ}}{D^{\prime} E^{\prime} \sin \theta \cos \phi \sin 80^{\circ}\left[2 E\left(E+E^{\prime} \cos \theta\right)+Q^{2}\right]}
\end{aligned}
$$

From Eq.(1.1) of Chapter 1, we know that the spin asymmetries are given by:

$$
\begin{gathered}
A_{1}=\nu \frac{M G_{1}}{W_{1}}-Q^{2} \frac{G_{2}}{W_{1}} \\
A_{2}=\sqrt{Q^{2}}\left(\frac{M G_{1}}{W_{1}}+\nu \frac{G_{2}}{W_{1}}\right) \\
94
\end{gathered}
$$


Substituting $M G_{1} / W_{1}$ and $G_{2} / W_{1}$ of equation (4.32) into equation (4.33) and with further simplification gives (see [71]):

$$
\begin{aligned}
A_{1}= & \frac{1}{D^{\prime}}\left(\frac{E-E^{\prime} \cos \theta}{E+E^{\prime}}+\frac{E^{\prime} \sin \theta \cos 80^{\circ}}{\left(E+E^{\prime}\right) \cos \phi \sin 80^{\circ}}\right) A_{180^{\circ}} \\
& +\frac{1}{D^{\prime}} \frac{E^{\prime} \sin \theta}{\left(E+E^{\prime}\right) \cos \phi \sin 80^{\circ}} A_{80^{\circ}} \\
A_{2}= & \frac{1}{D^{\prime}} \frac{\sqrt{Q^{2}}}{2 E}\left(A_{180^{\circ}}-\frac{\left(E-E^{\prime} \cos \theta\right) \cos 80^{\circ}}{E^{\prime} \sin \theta \cos \phi \sin 80^{\circ}} A_{180^{\circ}}-\frac{E-E^{\prime} \cos \theta}{E^{\prime} \sin \theta \cos \phi \sin 80^{\circ}} A_{80^{\circ}}\right)
\end{aligned}
$$

Rearranging the factors by common kinematic terms we arrive at:

$$
\begin{aligned}
& A_{1}=\frac{1}{D^{\prime}}\left(\frac{E-E^{\prime} \cos \theta}{E+E^{\prime}} A_{180^{\circ}}+\frac{E^{\prime} \sin \theta}{\left(E+E^{\prime}\right) \cos \phi} A_{180^{\circ}} \frac{\cos 80^{\circ}+A_{80^{\circ}}}{\sin 80^{\circ}}\right) \\
& A_{2}=\frac{1}{D^{\prime}} \frac{\sqrt{Q^{2}}}{2 E}\left(A_{180^{\circ}}-\frac{\left(E-E^{\prime} \cos \theta\right)}{E^{\prime} \sin \theta \cos \phi} A_{180^{\circ}} \frac{\cos 80^{\circ}+A_{8} 0^{\circ}}{\sin 80^{\circ}}\right)
\end{aligned}
$$

Where $D^{\prime}$ and $\epsilon$ are defined as in equation (4.27)

$A_{1}$ and $A_{2}$ from equation (4.35) are the spin asymmetries calculated from the measured asymmetries.

The extraction of the spin structure functions $g_{1}$ and $g_{2}$ follows from the asymmetries such that:

$$
\begin{aligned}
g_{1} & =\frac{F_{1}}{1+\gamma^{2}}\left(A_{1}+\gamma A_{2}\right) \\
g_{2} & =\frac{F_{1}}{1+\gamma^{2}}\left(\frac{A_{2}}{\gamma}-A_{1}\right)
\end{aligned}
$$

Where $\gamma=\sqrt{4 x^{2} M^{2} / Q^{2}}$ 


\section{CHAPTER 5}

\section{PION ASYMMETRY}

\subsection{Introduction}

As mentioned in section 4 of the previous chapter, it is very important to understand and perform several corrections in order to have clean physics asymmetry and meet with the goals of the experiment. One of such corrections is that of the positron-electron $\left(e^{+} e^{-}\right)$pair symmetric background primarily from $\pi^{0}$ decay which constitutes the main source of background in SANE. In creating positron-electron pairs, these processes provide additional electrons that can enter the BETA detector setup and are indistinguishable from an outgoing DIS electron. The background contribution is high at low energy [61]. A full understanding of this phenomena is necessary to get clean results for SANE as well as extend SANE's understanding of the kinematic region at low $x$. Figure 5.1 show charge-symmetric processes from $\pi^{0}$ decay simulated by using a SLAC $e^{+} e^{-}$parameterization [60]. From the plot it can be seen that there is a need to reduce the positron rates by increasing the energy threshold to $E^{\prime}>1.3 \mathrm{GeV}$ (see section 5.6.3 for details).

Figure 5.2 shows the background ratio for the various energy configurations.

There are three main sources of processes that generate target background. Even though other particles than $\pi^{0}$ decay into $e^{+} e^{-}$or $e^{-}$but they have small probabilities and so were neglected in the analysis. These processes are listed below: 


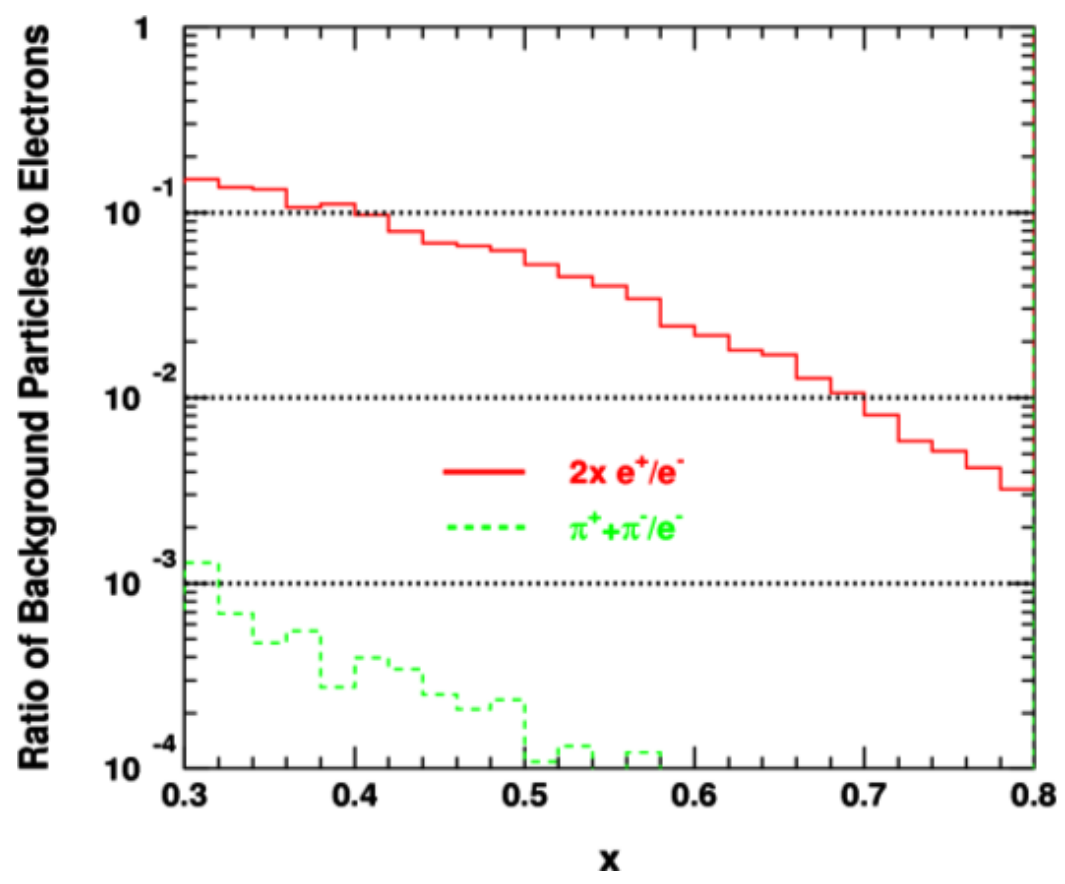

Figure 5.1

Ratio of Background Particles to Electron High at Low $x$

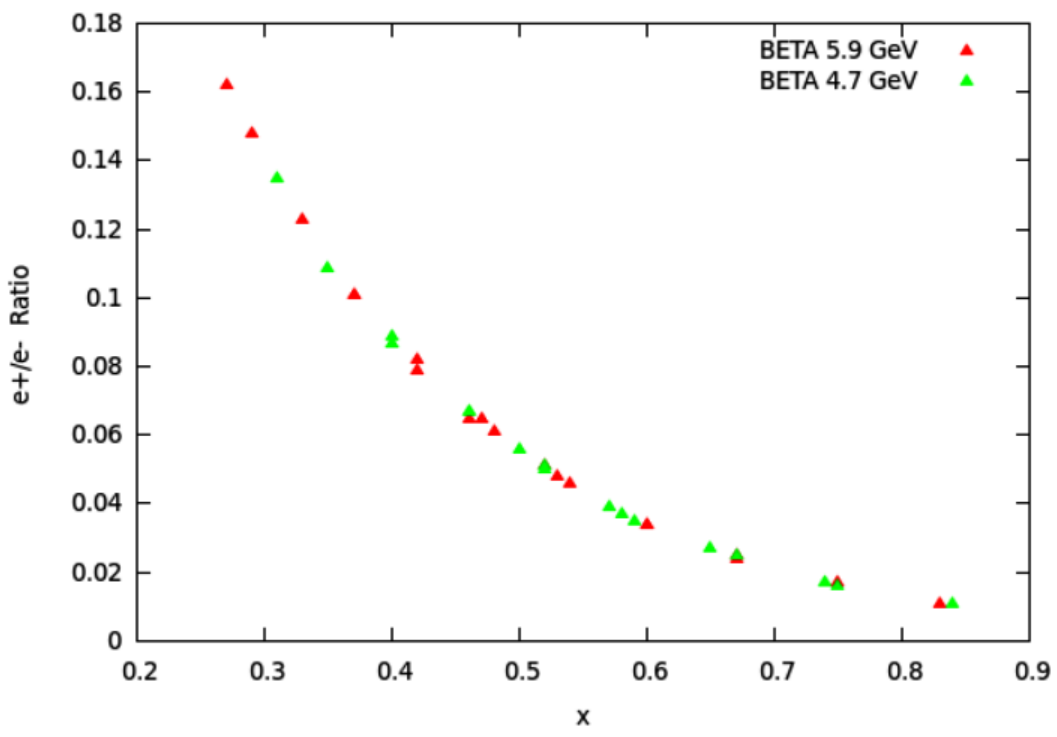

Figure 5.2

Charge Symmetric Background with SANE kinematics 
1. Bethe-Heitler (bremsstrahlung) into $e^{+} e^{-}$, however this process has a very small angle with respect to the beam, thus it is not a concern for SANE.

2. $\pi^{0}$ decays: $\pi^{0} \longrightarrow \gamma e^{+} e^{-}$and $\pi^{0} \longrightarrow \gamma \gamma$. This is the main concern for SANE.

3. Charged $\pi$ misidentified as electron.

\subsection{Pair-Sysmetric Background}

Pair symmetric processes create a background to inclusive electron scattering. In SANE, positron-electron $\left(e^{+} e^{-}\right)$pairs, which come primarily from neutral pion decay, constitute the main source of background. A neutral pion $\left(\pi^{0}\right)$ is created as the electron beam interacts with the target and quickly decays. The dominant decay of the $\pi^{0}$ is to two photons $\left(\pi^{0} \longrightarrow \gamma \gamma\right)$ with a branching ratio of $98.8 \%$ while most of the remaining $1.2 \%$ follows the Dalitz decay mode, $\left(\pi^{0} \longrightarrow \gamma e^{+} e^{-}\right)$. In creating $e^{+} e^{-}$pairs, these processes provide additional electrons that can enter our detector setup (BETA) and are indistinguishable from an outgoing DIS electron.

To extract the physics asymmetries and meet the experimental goals, the background contributions to the measured asymmetry must be well understood. Lepton pair production from bremsstrahlung photons are negligible for SANE. Furthermore, $\pi^{-}$and Kaons are not a significant background because they are removed by BigCal as they deposit less

energy than electrons. Also, the photons can interact in the tracker to produce $e^{+} e^{-}$pair. However, this is totally eliminated by a cut on the Čerenkov to remove double track events, as discussed in section 4.1.2. 


\subsubsection{Double Track Background from the Tracker}

The tracker that was placed between the target and the Čerenkov was found to increase the background. This was due basically to double tracks background coming from gamma conversions in the tracker. A simulation was done using GEANT to better understand the effect of the tracker on the background contamination. Furthermore, the Čerenkov ADC signal was used to cut these double tracks background from gamma conversions in the tracker.

In the stimulation $\pi^{0}$ events were generated when the tracker was 'In' (place between the target and the Čerenkov) and then when it was taken 'Out'. The EPC code [77] with the Wiser parameterization [78] of inclusive pion bremsstrahlung cross section were used for the generation and simulation of the pion events. The events were selected as follows:

1. Two photon events: Two cluster events with energies above $0.6 \mathrm{GeV}$ with no Čerenkov hit (or signal) for both clusters.

2. One Cluster event: One cluster event with energy above $0.6 \mathrm{GeV}$ with no Cerenkov hit for cluster.

3. $e^{-} e^{+}$: Two cluster events with energies above $0.6 \mathrm{GeV}$ with Čerenkov hit for both clusters.

4. One $e^{-}$or $e^{+}$: One cluster event with energy above $0.6 \mathrm{GeV}$, that is Čerenkov hit for cluster.

See section 5.4 for the definition of these cuts. Figure 5.3 depicts these scenarios. We focused on the single lepton and pair events. For each case we found that: for the $e^{-}$or $e^{+}$ events, the ratio $\left(N_{\text {out }} / N_{\text {in }}\right)$ was calculated to be about $68 \% \pm 1 \%$ whereas for the $e-e+$ events, $\left(N_{\text {out }} / N_{\text {in }}\right)$ ratio was calculated to be about $64 \% \pm 2 \%$. Where $N_{\text {out }}$ is the count when the tracker is out and $N_{i n}$ is the count when the tracker is in. Thus it can be seen that 
the tracker contributed about $30 \%$ of the background since BETA is charge insensitive, it does do not distinguish between $e^{-}$or $e^{+}$.

Figure 5.4 shows the Čerenkov ADC signal as a function of the number of tracks where the Čerenkov ADC window cut is defined by the events between the two vertical lines. This picture shows the necessity of the Čerenkov cut, the purpose of which is to remove the background of pairs produced by photons outside of the central region of the target magnetic field. The black curve shows the sum of all the events. The blue curve shows the relative yield for events that originated with a scattered electron. The red curve shows the background events.

\subsection{Trajectory of a $e^{+} e^{-}$pair in SANE}

In this section an example is presented to give a practical picture of the trajectory of a $e^{+} e^{-}$pair in SANE as shown in Figure 5.5 and Figure ??.

The example in Figure 5.5 and Figure 5.6 show how each particle positron (blue), electron (red) each with momentum $700 \mathrm{MeV}$. The pair starts out in a direction of $40^{\circ}$ along the BETA axis. There is vertical deflection of $11.93^{\circ}$ which translates to $71 \mathrm{~cm}$ in BigCal. The cluster energy $700 \mathrm{MeV}$ corresponds to $x=0.2$ for DIS electrons at $40^{\circ}$.

\subsection{Calculating the Pair-Symmetric Background Asymmetry}

In order to calculate the $\pi^{0}$ asymmetry the $\pi^{0}$ events are identified by reconstructing the $\pi^{0}$ mass from energy deposited in BigCal from events with two clusters produced by neutral particles. The identification of $\pi^{0}$ is done by placing a cut on $\pi^{0}$ mass and using the 

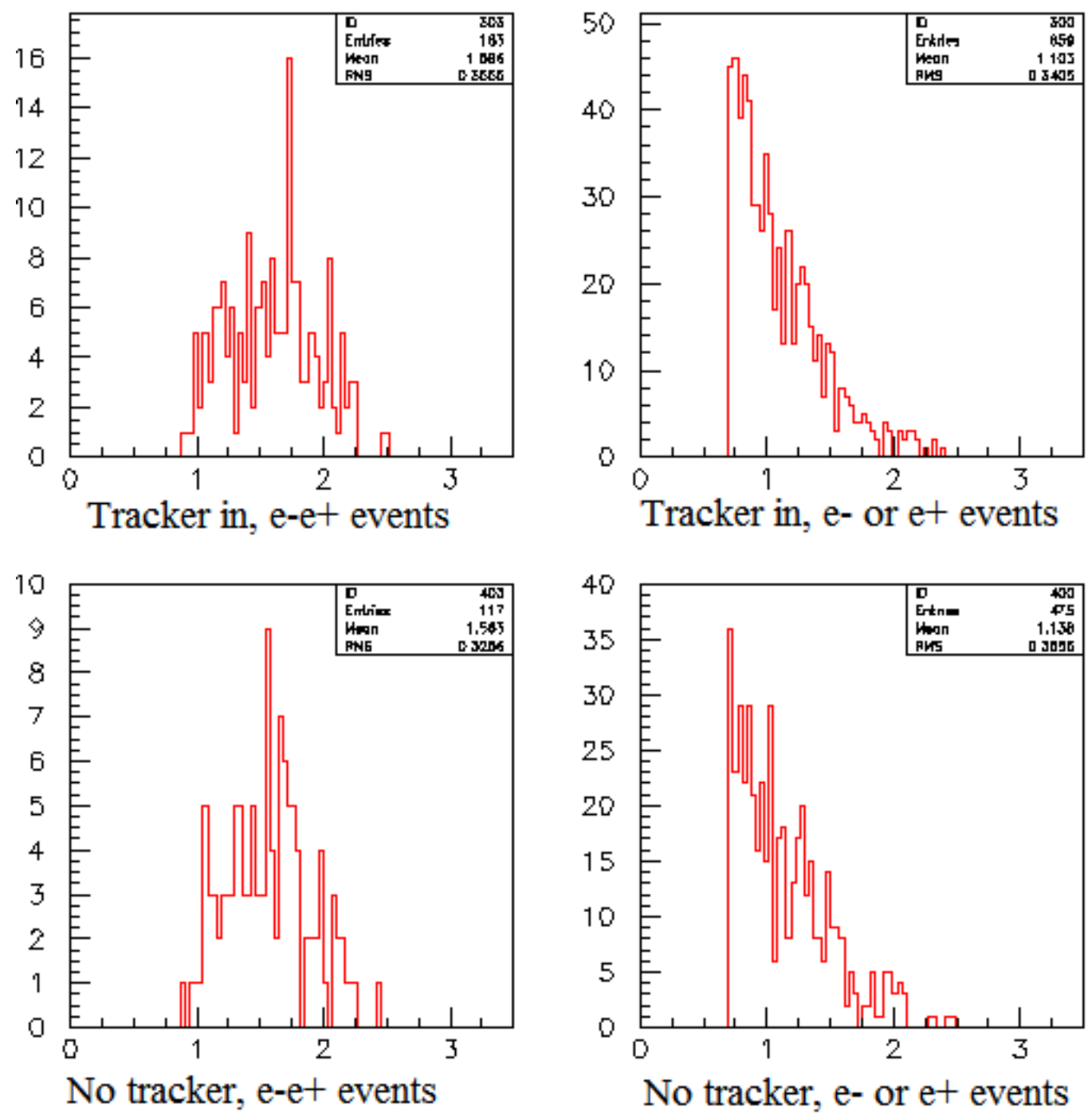

Figure 5.3

Tracker Analysis, When Tracker is In/Out 


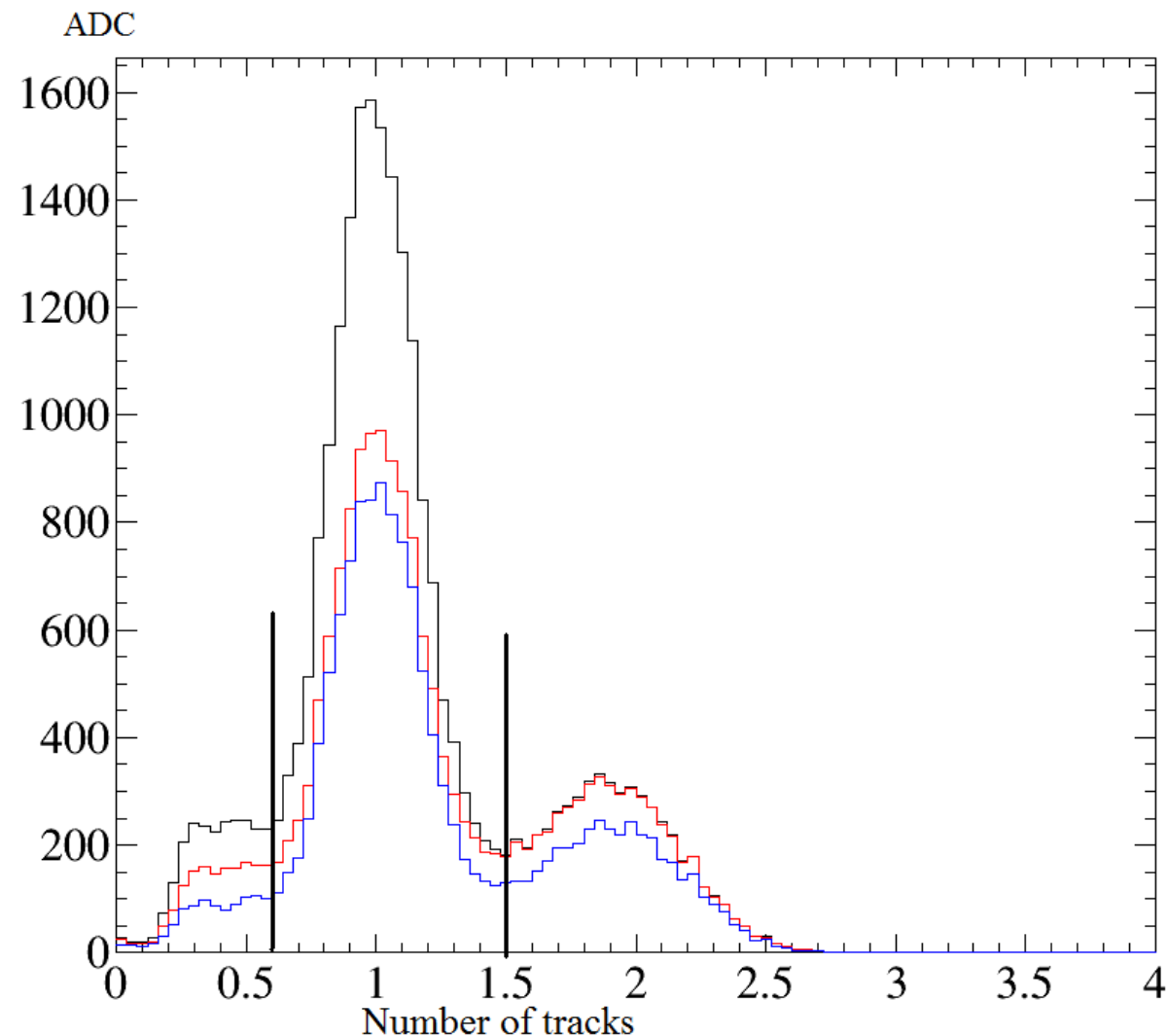

Figure 5.4

Simulated Čerenkov Response with Double Track ADC Spectrum 


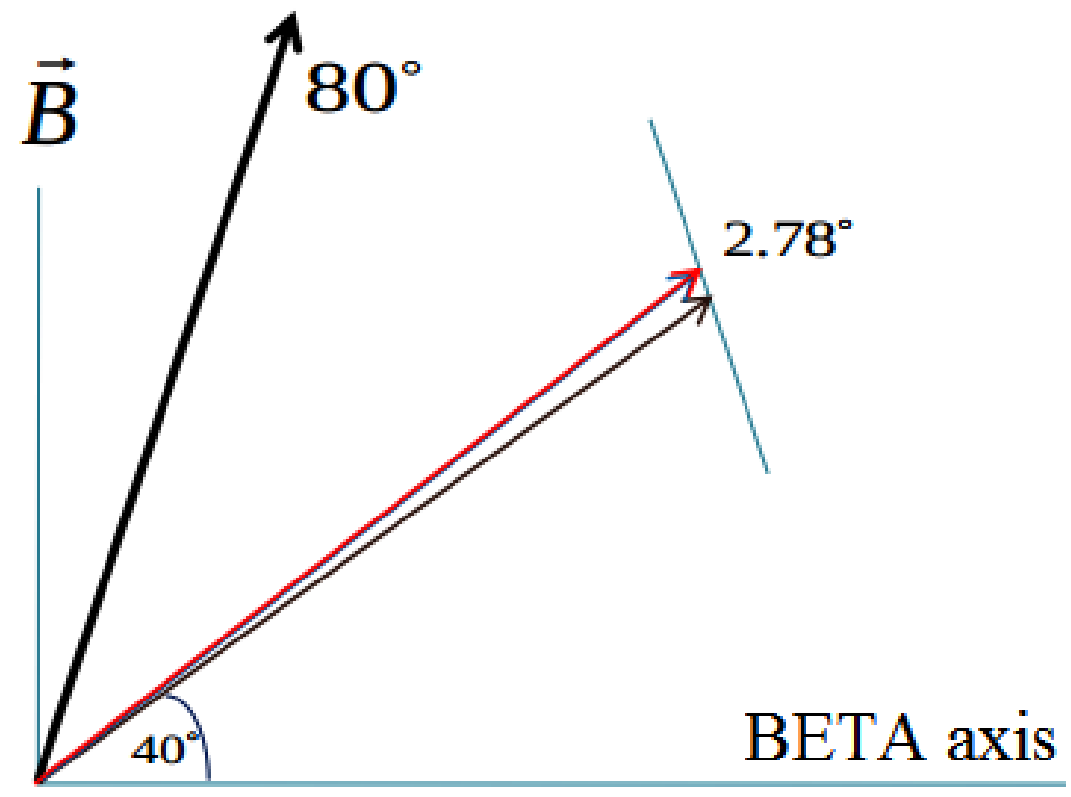

Figure 5.5

Red-electron, blue-positron) starting direction of $40^{\circ}$ along the BETA axis

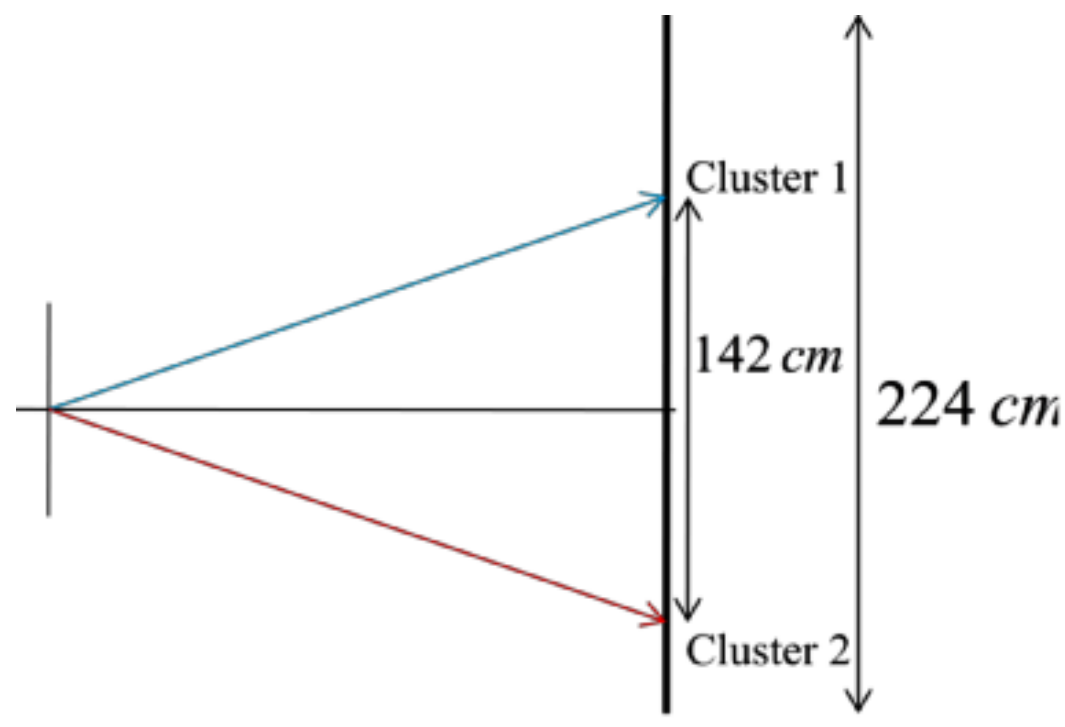

Figure 5.6

Vertical Deflection and Translation in the BigCal 
reconstructed energy cut: $E>0.6 \mathrm{GeV}$. At high energy, $\pi^{0}$ have low contribution, whereas below $0.5 \mathrm{GeV}$ the neural network does not work well [75].

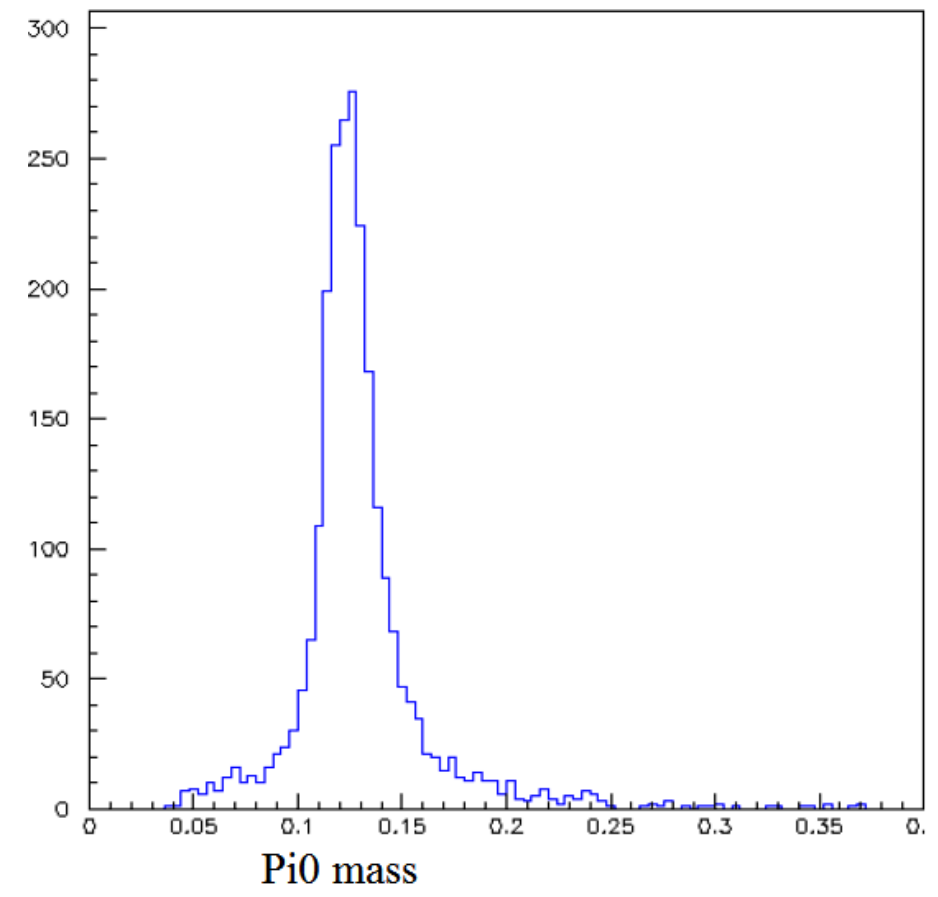

Figure 5.7

\section{Reconstructed $\pi^{0}$ Mass from Two Neutral Cluster Particles}

The raw $\pi^{0}$ asymmetry is calculated as follows:

$$
A_{\text {raw }}=\frac{\frac{N_{+}}{C_{+}}-\frac{N_{-}}{C_{-}}}{\frac{N_{+}}{C_{+}}+\frac{N_{-}}{C_{-}}},
$$

where $N^{+(-)}$is the number of counts with helicity positive (negative), $C^{+(-)}$is the incoming charge accumulated during the event with positive (negative) helicity. 


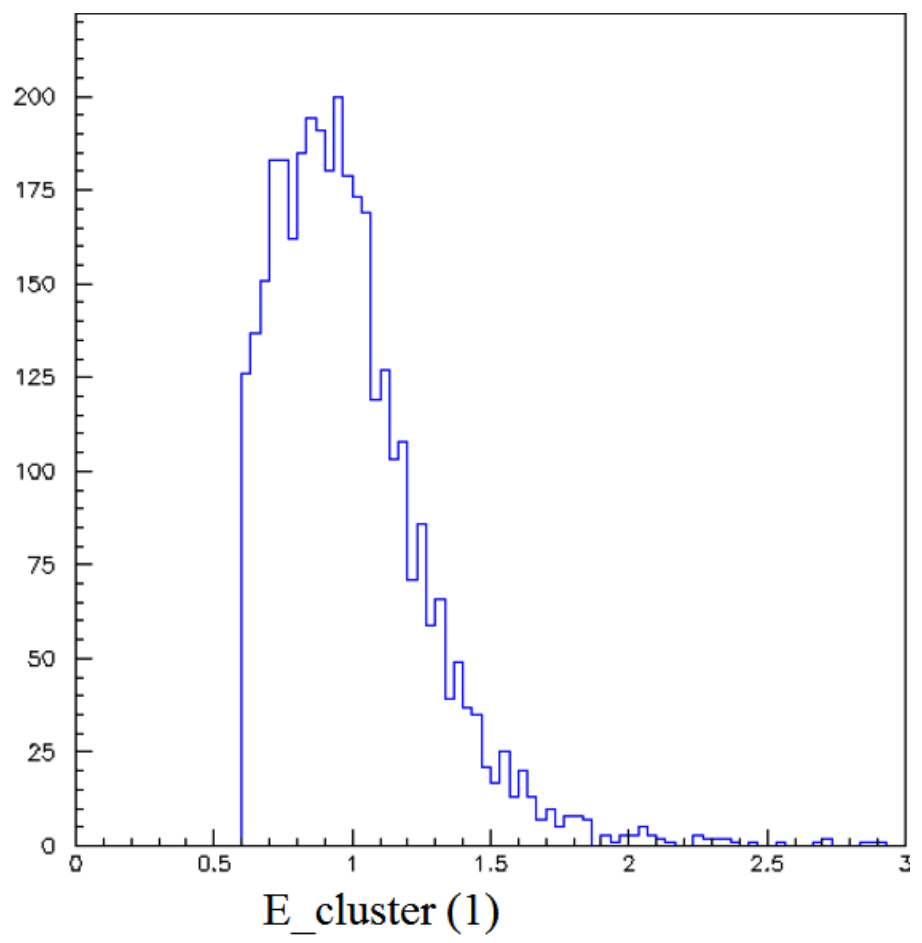

Figure 5.8

E(1) of Run 73001 


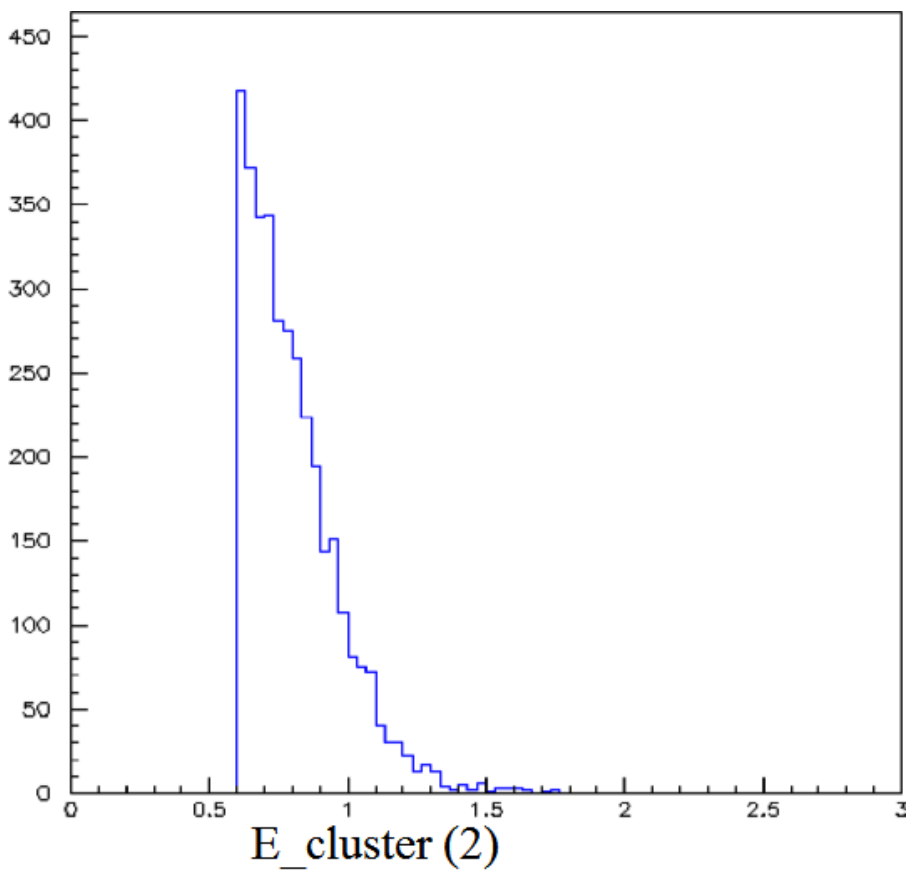

Figure 5.9

$E(2)$ of Run 73001 


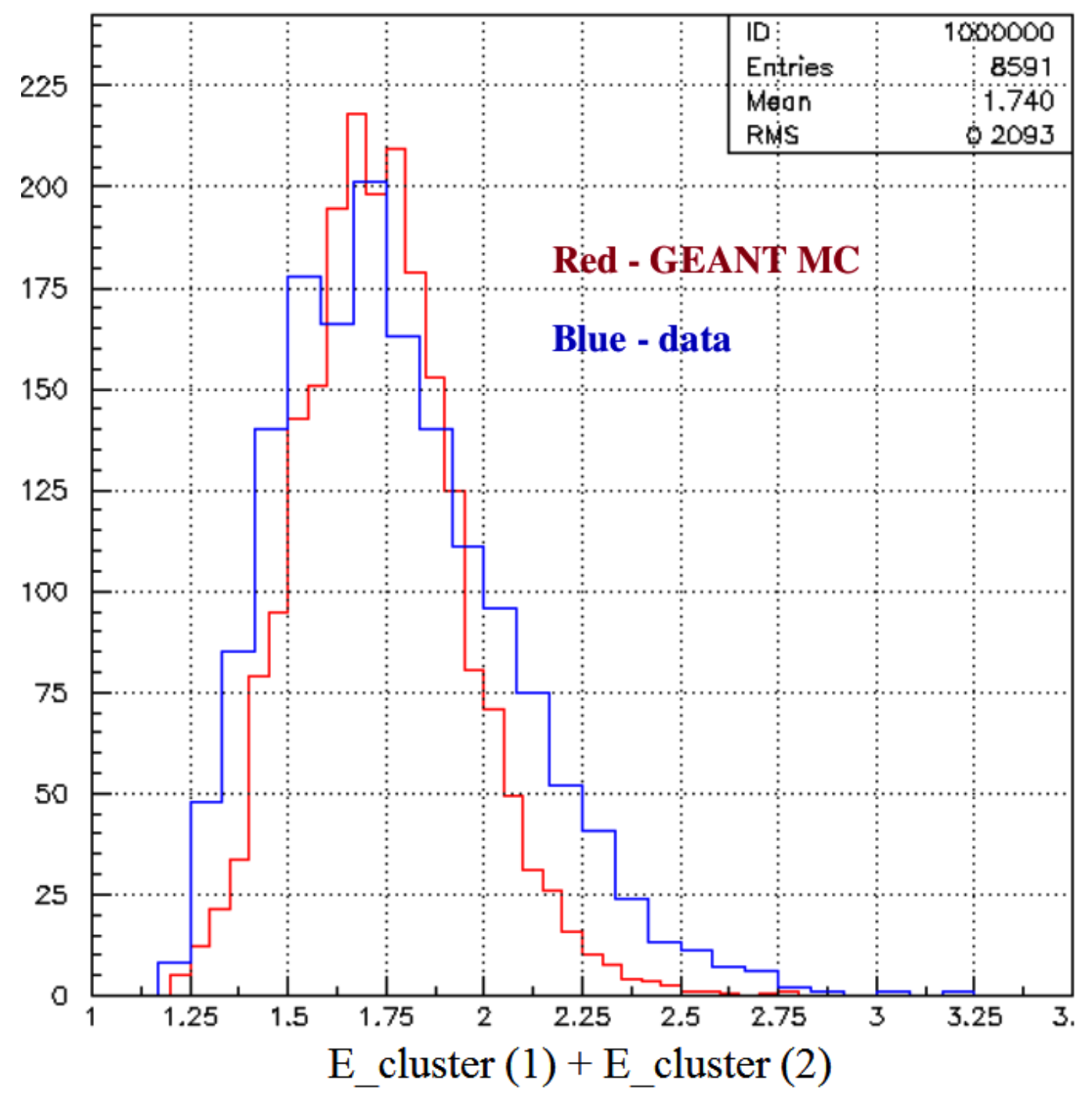

Figure 5.10

Comparing the MC (red plot) with Data 
The physics asymmetry is obtained by considering the beam and target, $P_{b}$ and $P_{t}$ respectively:

$$
A_{\text {physics }}=\frac{1}{P_{b} P_{t}} A_{\text {raw }}
$$

Moreover, since the pion asymmetry can only come from scattering on polarized nucleon, the physics asymmetry needs to include a dilution factor $1 / f$ with $f \sim 0.14$ (see section 5.6.1 on how $f$ is obtained). Hence equation (5.2) becomes:

$$
A_{\text {physics }}=\frac{1}{f P_{b} P_{t}} A_{\text {raw }}
$$

As shown on Figure 5.7, Figure 5.8 and Figure 5.9, we start with the reconstruction of the $\pi^{0}$ mass from events with two clusters produced by neutral particles and then we identify $\pi^{0}$ by putting a cut on the $\pi^{0}$ mass. The $\pi^{0}$ mass plot is shown in Figure 5.7.

The following cuts were used:

1. The cut: $e_{\_} r>0.6$ is used to present the energy in GeV of the indexed clusters in the event is $>0.6 \mathrm{GeV}$. The stability of the cut was checked by plotting the average asymmetry versus run numbers for the various cuts as shown in figure ??. Figure ?? further shows the stability of the average total $\pi^{0}$ asymmetry for various energy cuts.

2. The cut: $n c l u s t=2$ is used to denote the number of clusters in an event is equal to two.

3. The cut: $c e r \_h=0$ is used for events with no hit in the Čerenkov (that is no Čerenkov signal).

4. The cut: i_helicity $>0($ or $<0)$ to denote the beam helicity as indicated by the trigger supervisor.

5. The distance $(\mathrm{cm})$ cut was such that $20 \mathrm{~cm}<$ distance between clusters $<80 \mathrm{~cm}$.

Figure 5.11 shows the stability of the asymmetry to energy cuts. Furthermore, on the same picture is plotted the product of the beam and target polarization, $P_{b}, P_{t}$ (red 
crosses) where $P_{b} * P_{t}$ has been multiplied by a factor of 2 to fit on the plot. In addition, the $\pi^{0}$ asymmetries are combined by groups of runs with the same sign of the product $P_{b} * P_{t}$. Figure 5.12 shows the total average asymmetry for all cuts versus the energy cuts. Table 5.1 shows total average asymmetry for the various energy cuts. Table 5.2 shows the $\pi^{0}$ Asymmetries Combined by Groups of Runs with the Same Sign of the Product of $P_{b} * P_{t}$.

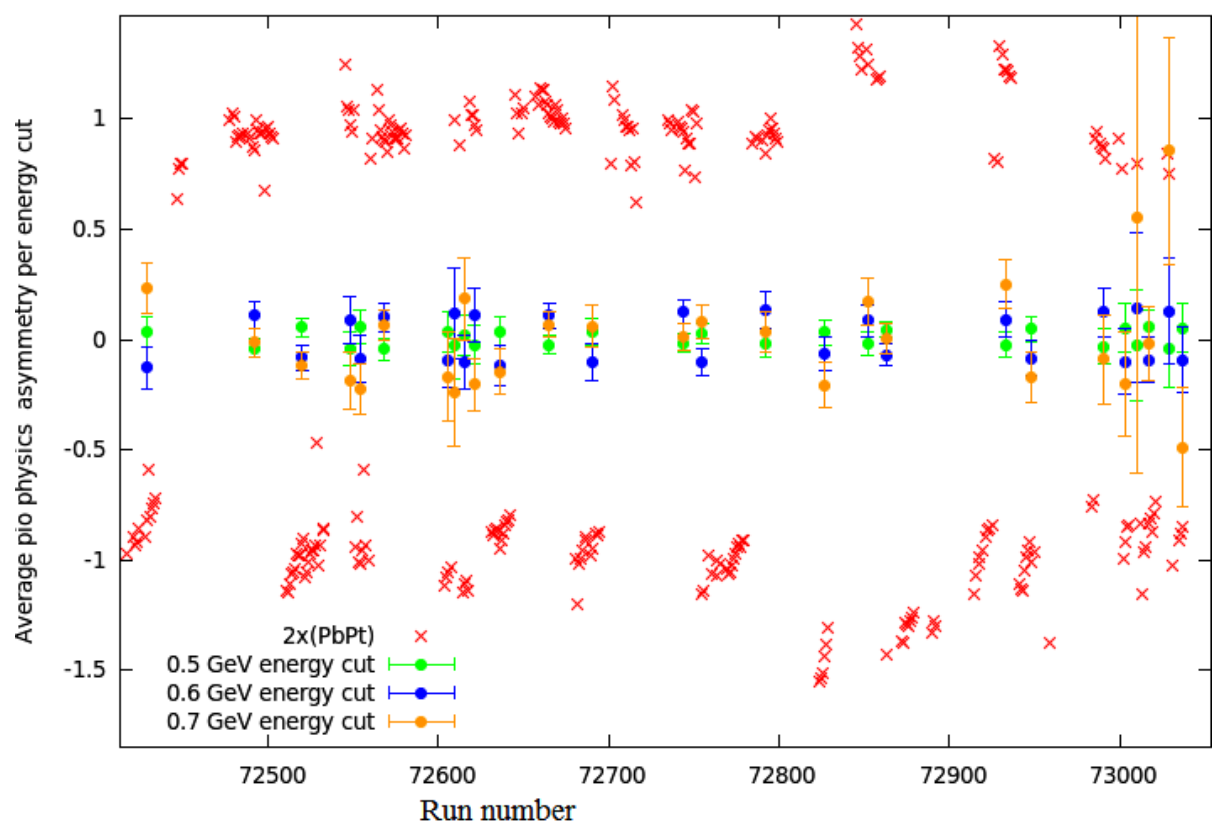

Figure 5.11

Average Asymmetry Versus Run Numbers for the Energy Cuts

The plot in Figure 5.13 shows the average physics $\pi^{0}$ asymmetry with the line $f=0.14$ being the dilution factor. The blue points indicate the average physics asymmetry while the red points indicate the product of the beam and target polarization $\left(P_{b} P_{t}\right)$, which were 


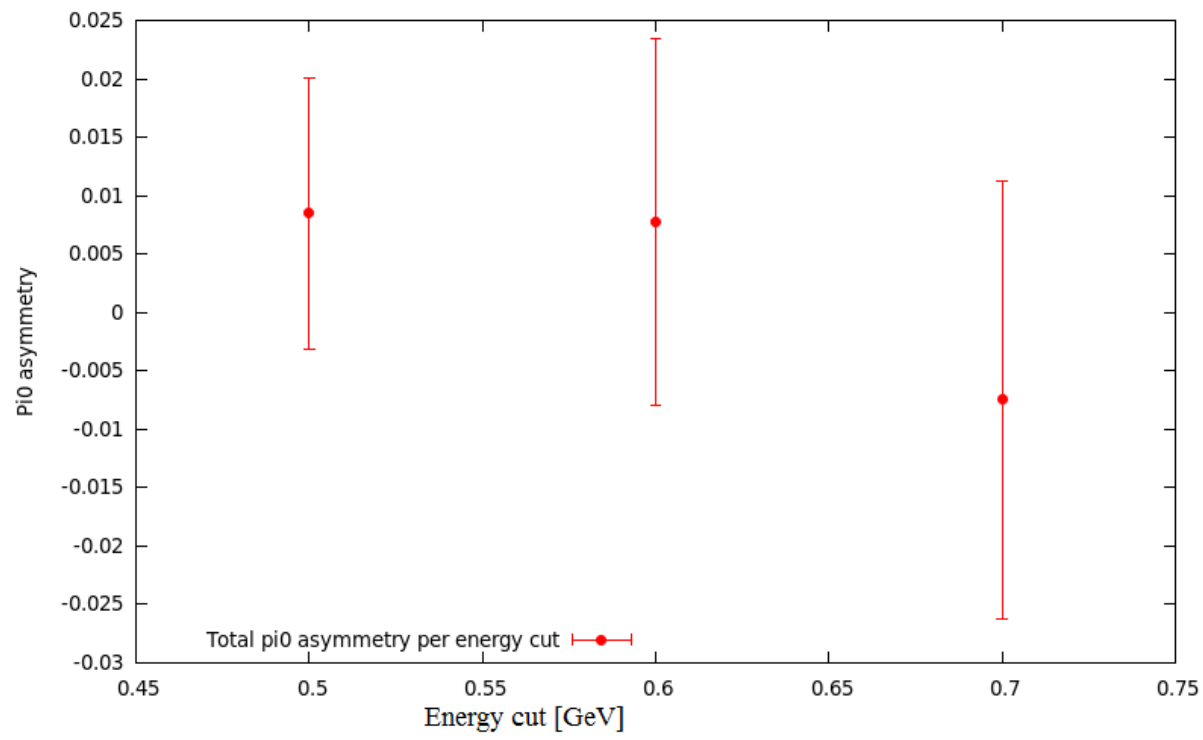

Figure 5.12

Total Average Asymmetry for All Cuts Versus Energy Cuts

Table 5.1

Total Average Asymmetry for the Various Energy Cuts

\begin{tabular}{ccc}
\hline Energy Cut $(\mathrm{GeV})$ & Total Asymmetry & Total Asymmetry Error \\
\hline 0.5 & 0.0085 & 0.0116 \\
0.6 & 0.0078 & 0.0157 \\
0.7 & -0.0075 & 0.0188 \\
\hline
\end{tabular}


averagely $73 \%$ and $69 \%$ respectively. In Figure 5.13 the product $P_{b} P_{t}$ has been scaled by a factor of two so as to fit in the same plot.

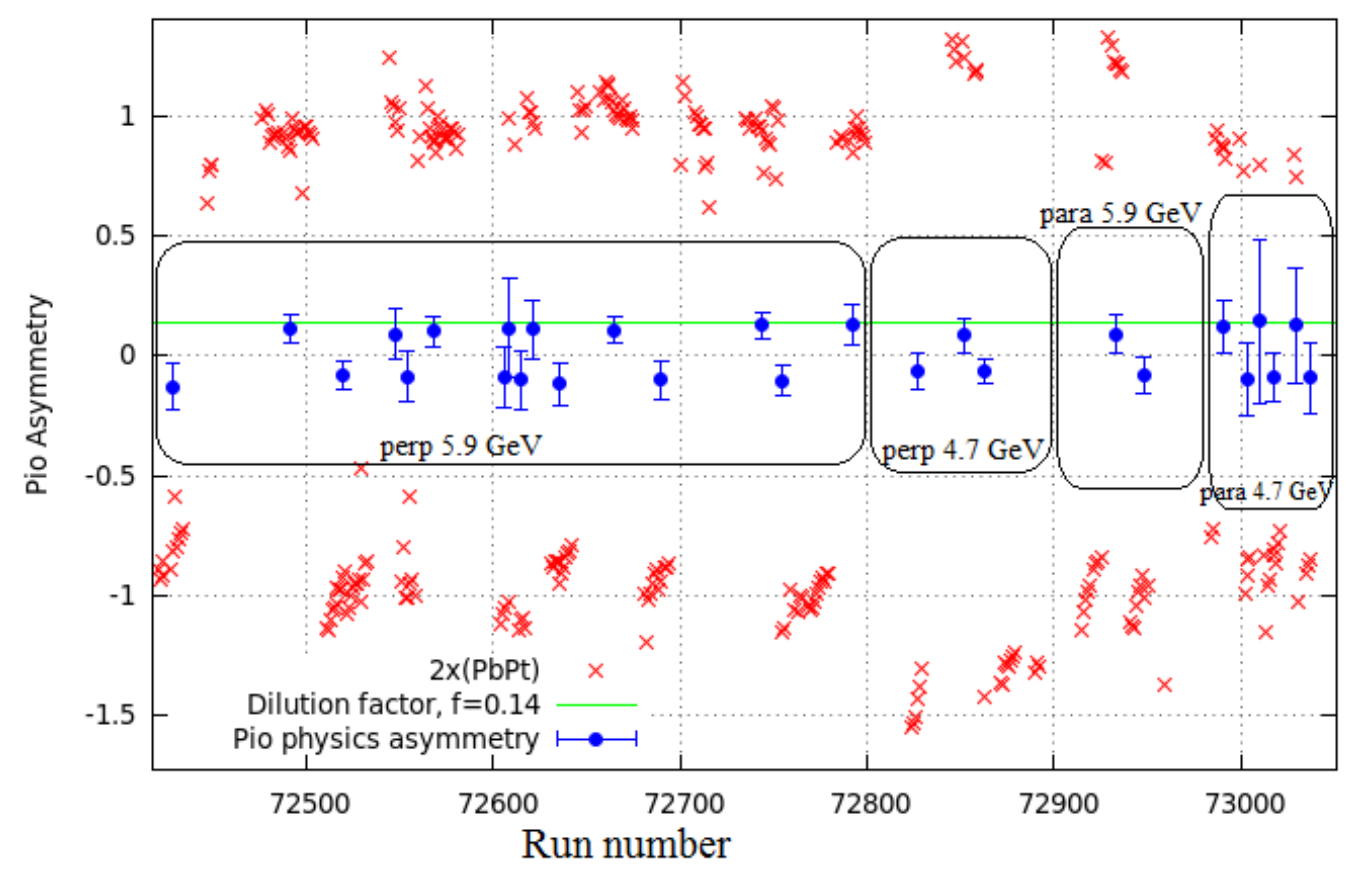

Figure 5.13

Average $\pi^{0}$ Physics Background Asymmetry (Blue Circles)

Figure 5.13 shows that the total $\pi^{0}$ asymmetry is consistent with zero. Table 5.3 shows $\pi^{0}$ asymmetries combined by groups of runs with the same sign of the product $P_{b} * P_{t}$. The run numbers shown correspond to the first run of each group. The data shows some agreement with the SLAC E155 results. This will be used in section 5.6 to calculate the 
Table 5.2

Pion Asymmetries for the Various Energy and Angle Orientations

\begin{tabular}{ccc}
\hline Energy $(\mathrm{GeV})$ & Orientation & Physics asymmetry \\
\hline 5.9 & $80^{\circ}$ & $0.0206 \pm 0.0193$ \\
4.7 & $80^{\circ}$ & $-0.0137 \pm 0.0393$ \\
5.9 & $180^{\circ}$ & $-0.0207 \pm 0.0481$ \\
4.7 & $180^{\circ}$ & $-0.0180 \pm 0.0568$ \\
\hline$\left.<A_{\pi^{0}}\right\rangle$ & & $0.0078 \pm 0.0157$ \\
\hline
\end{tabular}

background dilution. Table 5.2 shows the pion asymmetries for the various energy and angle orientations

$$
\begin{aligned}
A\left(180^{\circ}\right) \text { for } S A N E & =-0.020 \pm 0.04 \\
A_{A L L}\left(180^{\circ}\right) \text { for } S L A C & =-0.022 \pm 0.002
\end{aligned}
$$

Therefore we can conclude that the total combined asymmetry of the neutral pion measured in SANE is consistent with zero and the $\pi^{0}$ contribution is more of a background dilution rather than an asymmetry.

\subsection{Systematic Error in Background Correction}

Even though the neutral pion data show an asymmetry consistent with zero with errors, the errors of this result will propagate to the systematic uncertainties of the background corrected asymmetries of about $10 \%$. 
Table 5.3

$\pi^{0}$ Asymmetries Combined by Groups of Runs with the Same Sign of the Product of $P_{b} * P_{t}$

\begin{tabular}{cccc}
\hline Run Number & Runs per group & Average asymmetry & Error \\
\hline 72429 & 12 & -0.1280435 & 0.094315 \\
72492 & 27 & 0.1104841 & 0.057759 \\
72520 & 23 & -0.0815739 & 0.057612 \\
72548 & 6 & 0.0885419 & 0.105221 \\
72554 & 8 & -0.0884082 & 0.105020 \\
72568 & 20 & 0.1004343 & 0.064884 \\
72606 & 4 & -0.0931135 & 0.126911 \\
72609 & 2 & 0.1169479 & 0.205036 \\
72615 & 4 & -0.1018342 & 0.121171 \\
72621 & 5 & 0.1110645 & 0.120611 \\
72636 & 12 & -0.1175212 & 0.090736 \\
72665 & 23 & 0.1074975 & 0.054486 \\
72690 & 12 & -0.1025327 & 0.081327 \\
72744 & 27 & 0.1269283 & 0.055797 \\
72755 & 18 & -0.1034831 & 0.063189 \\
72792 & 12 & 0.1316201 & 0.085218 \\
72827 & 6 & -0.0616518 & 0.076158 \\
72852 & 9 & 0.0867471 & 0.071472 \\
72863 & 21 & -0.0679715 & 0.050460 \\
72933 & 9 & 0.0915698 & 0.078221 \\
72948 & 12 & -0.0831270 & 0.076641 \\
72991 & 8 & 0.1223320 & 0.109845 \\
73003 & 4 & -0.0986898 & 0.150569 \\
73010 & 1 & 0.1440228 & 0.341566 \\
73017 & 9 & -0.0918755 & 0.101957 \\
73029 & 2 & 0.1284352 & 0.241060 \\
73037 & 4 & -0.0932524 & 0.147693 \\
\hline & & &
\end{tabular}


To obtain the contribution of the error due to the uncertainty in the background and background asymmetry we proceed as follow: Taking the background into consideration, the measured asymmetry $A_{m}$ is:

$$
A_{m}=\frac{N^{+}-N^{-}+N_{b}^{+}-N_{b}^{-}}{N^{+}+N^{-}+N_{b}^{+}+N_{b}^{-}}
$$

Setting $N_{m}=N^{+}+N^{-}+N_{b}^{+}+N_{b}^{-}=N+N_{b}$ where $N_{m}$ is the measured count, and $N_{b}$ the background count. With $N=N^{+}+N^{-}$and $N_{b}=N_{b}^{+}+N_{b}^{-}$

$A_{m}$ becomes:

$$
\begin{aligned}
A_{m} & =\frac{\frac{N^{+}-N^{-}}{N^{+}+N^{-}}\left(N^{+}+N^{-}\right)+\frac{N_{b}^{+}-N_{b}^{-}}{N_{b}^{+}+N_{b}^{-}}\left(N_{b}^{+}+N_{b}^{-}\right)}{N_{m}} \\
& =\frac{A\left(N^{+}+N^{-}\right)+A_{b}\left(N_{b}^{+}+N_{b}^{-}\right)}{N_{m}} \\
& =\frac{\left(N_{m}-N_{b}\right) A+N_{b} A_{b}}{N_{m}},
\end{aligned}
$$

where $A$ and $A_{b}$ are the physics asymmetry and background asymmetry respectively given by:

$$
A=\frac{N^{+}-N^{-}}{N^{+}+N^{-}} ; \quad A_{b}=\frac{N_{b}^{+}-N_{b}^{-}}{N_{b}^{+}+N_{b}^{-}}
$$

with $N^{+}+N^{-}=N=N_{m}-N_{b}$ and $N_{b}^{+}+N_{b}^{-}=N_{b}$, we can thus rewrite the physics asymmetry $A$ as:

$$
A=\frac{A_{m}-f_{b} A_{b}}{1-f_{b}}
$$

where $f_{b}=\frac{N_{b}}{N_{m}}$ is the background dilution which is the ratio of the background count to the measured counts. The error propagation is computed using the formula:

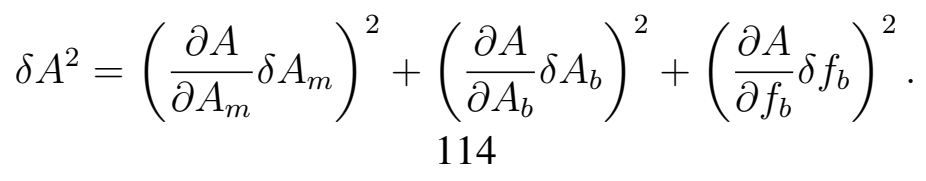


Further

$$
\frac{\partial A}{\partial A_{m}}=\frac{1}{1-f_{b}} ; \quad \frac{\partial A}{\partial A_{b}}=\frac{f_{b}}{1-f_{b}} ; \quad \frac{\partial A}{\partial f_{b}}=\frac{A_{m}-A_{b}}{\left(1-f_{b}\right)^{2}} .
$$

Therefore, substituting equation (5.10) into equation (5.9), we obtain:

$$
\delta A^{2}=\frac{1}{\left(1-f_{b}\right)^{2}}\left[\left(\delta A_{m}\right)^{2}+\left(f_{b} \delta A_{b}\right)^{2}+\left(\frac{A_{m}-A_{b}}{1-f_{b}}\right)^{2}\left(\delta f_{b}\right)^{2}\right] .
$$

Now $A_{e^{+} / e^{-}}=A_{b} \sim 0$

$$
\delta A^{2}=\frac{A^{2} f_{b}^{2}}{\left(1-f_{b}\right)^{2}}\left(\frac{\partial A_{m}}{A f_{b}}\right)^{2}+\frac{A^{2} f_{b}^{2}}{\left(1-f_{b}\right)^{2}}\left[\left(\frac{\delta A_{b}}{A}\right)^{2}+\left(\frac{\delta f_{b}}{f_{b}}\right)^{2}\right] .
$$

The first term is statistical, hence it is dropped, while, the second term is treated as a systematic error. Thus the relative systematic error of the corrected asymmetries due to $\delta A_{b}$ is:

$$
\frac{\delta A}{A}=\frac{f_{b}}{\left(1-f_{b}\right)}\left[\left(\frac{\delta A_{b}}{A}\right)^{2}+\left(\frac{\delta f_{b}}{f_{b}}\right)^{2}\right]^{\frac{1}{2}} .
$$

Where $\frac{\delta A_{b}}{A}$ is the uncertainty of the background asymmetry, $\frac{\delta f_{b}}{f_{b}}$ is the error of the background dilution and $\frac{\delta A}{A}$ is the systematic error.

\subsection{Calculating $A_{b}$ and $f_{b}$}

In the analysis of the background asymmetry $A_{b}$ and background dilution $f_{b}$ we have made used of the SLAC $A_{\|}$and $A_{\perp}$ data but suitably modified for the SANE kinematics. For example, SANE $\left(A_{180}\right)$ shows agreement with SLAC $\left(A_{\|}\right)$though with larger error bars. Furthermore, SANE $\left(A_{80}\right)$ is not zero while SLAC $\left(A_{\perp}\right)$ though consistent with zero but has some error.

It should be noted that SLAC $\left(A_{\|}\right)$is a statistical weighted average of $\pi^{+}$and $\pi^{-}$ asymmetries whereas, SANE $\left(A_{180}\right)$ is a direct $\pi^{0}$ asymmetry. The SLAC experiment 
made use of magnetic spectrometers to measure the actual background rates with high $e^{+}$ rejection efficiency. In SANE, BETA was charge insensitive with an open configuration and detected both charges such that for each $e^{+}$detected there is a corresponding $e^{-}$. It therefore made sense to use the more precise SLAC background results in our analysis.

Data from the SLAC experiments E155 and E155x were used in the analysis presented in this section. The SLAC E155 took dedicated data on longitudinal pion asymmetry while E155x took transverse asymmetry data. The kinematics were at high transverse momentum $\left(P_{T}\right)$ where

$$
P_{T}=P_{\pi} \sin \theta
$$

where $\theta$ is the angle the detector makes with the beam line and $P_{\pi}$ is the pion momentum. Therefore, by knowing just the detector's measured variables, momentum and angle relative to the beam, the $P_{T}$ of any detected particle can be calculated no matter how it was produced.

The asymmetry data of $\pi^{+}$and $\pi^{-}$collected from E155 [84] and E155x [87] were combined and fitted as functions of the pion transverse momentum. This is possible because the SANE and SLAC experiments were at very comparable $P_{T}$. In addition, at high $P_{T}$ the inclusive cross sections scale depend almost exclusively on $P_{T}$ [86]. This therefore gives us a concrete basis to parametrize the SLAC asymmetries in terms of $P_{T}$ to compare with the SANE data. Table ?? illustrates the fact that SANE and SLAC were at very comparable $P_{T}$, where the large angle used by SANE compensates for lower detected pion momentum than SLAC.

compT 
Table 5.4

SANE and SLAC experiments were at comparable $P_{T}$

\begin{tabular}{cccc}
\hline Experiment & $P_{\text {pion }}(\mathrm{GeV} / \mathrm{c})$ & $\theta\left(^{\circ}\right)$ & $P_{T}(\mathrm{GeV} / \mathrm{c})$ \\
\hline SANE & 1.5 & 30 & 0.75 \\
SANE & 1.167 & 40 & 0.75 \\
SANE & 0.98 & 50 & 0.75 \\
SLAC & 15.6 & 2.75 & 0.75 \\
\hline
\end{tabular}

\subsection{1 $A_{b}$ for SANE's $A_{180^{\circ}}$ Configuration}

The data from table 2 of reference [84] for identified pions were used to make the plots in this subsection. In this table the photon endpoint energy is $48.35 \mathrm{GeV}$ and the errors are statistical only.

Figure 5.14 and Figure 5.15 show the asymmetries for $\pi^{+}$and $\pi^{-}$plotted versus $P_{T}$ for the various angles that were used in the E155 experiment, $2.75^{\circ}$ and $5.5^{\circ}$. Data from table 2 of [84] was used to make this plot.

The plots (Figure 5.14, Figure 5.15 and Figure 5.16)show that:

1. The asymmetries for $\pi^{+}$and $\pi^{-}$have similar shapes.

2. At lower angle, $2.75^{\circ}$ and $P_{T}$, the $\pi^{+}$and $\pi^{-}$asymmetries are slightly equal though the $\pi^{+}$asymmetries have larger error bars.

3. At $5.5^{\circ}$, and as $P_{T}$ increases the $\pi^{+}$and $\pi^{-}$asymmetries are not equal as was the case at $2.75^{\circ}$. They tend to diverge as the $P_{T}$ is increased.

4. The data seem to depend only on $P_{T}$ as expected.

5. There are more $e^{-}$asymmetry data points at high $P_{T}$.

6. According to the E155 published paper [84], the identified pion results have large statistical errors. This might be due to the large uncertainty in the dilution $\left(f^{\prime}\right)$. 


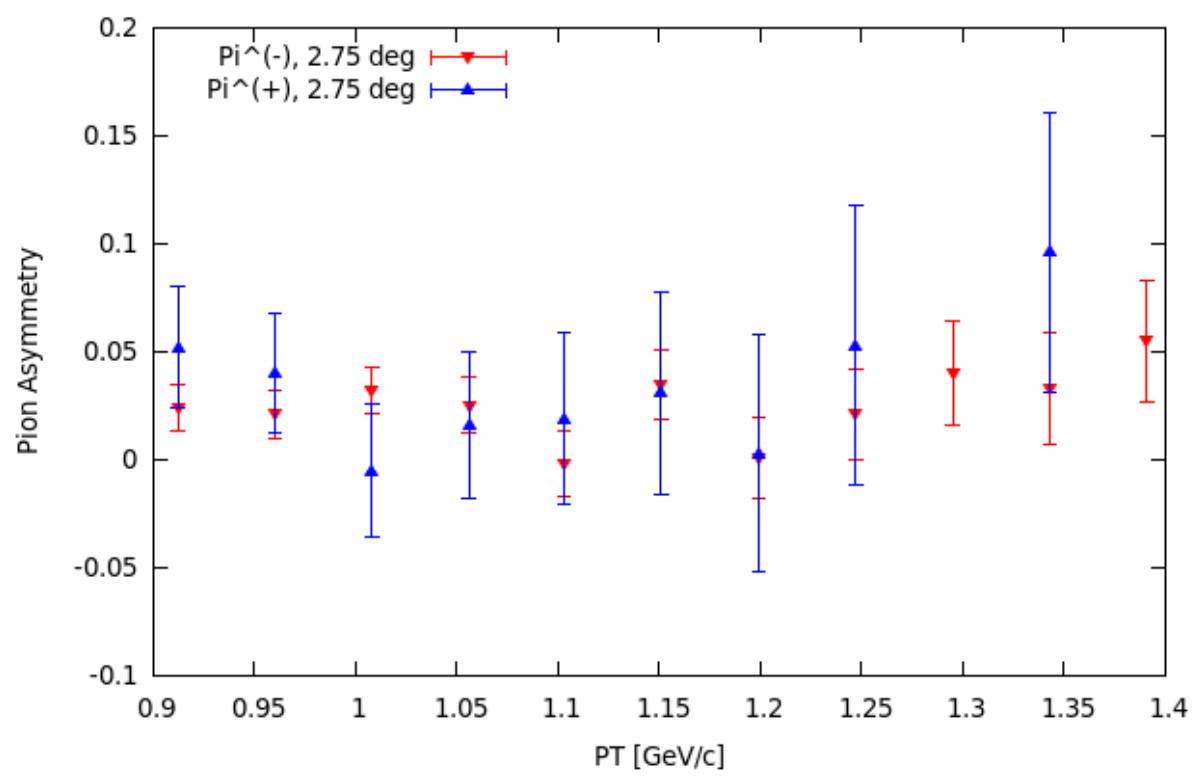

Figure 5.14

Plots of $\pi^{+}$(Blue) and $\pi^{-}$(Red) Asymmetries Versus $P_{T}$

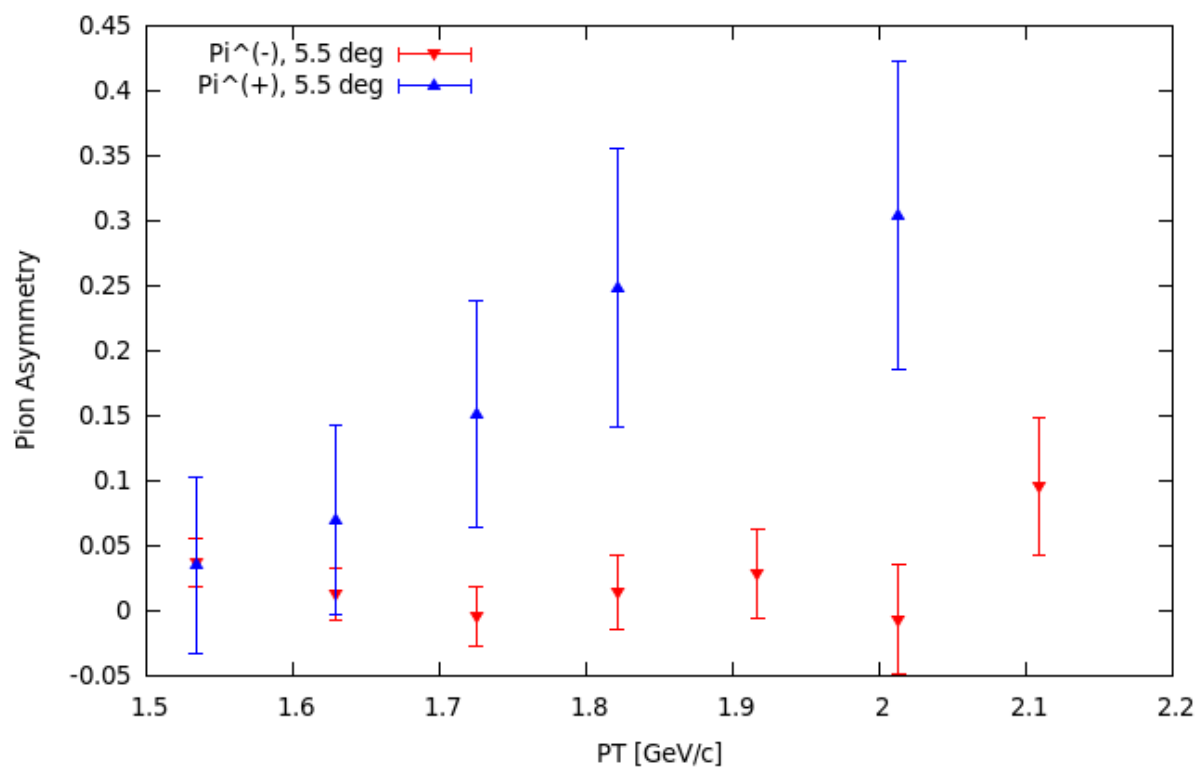

Figure 5.15

Plots of $\pi^{+}$(Blue) and $\pi^{-}$(Red) Asymmetries Versus $P_{T}$ 


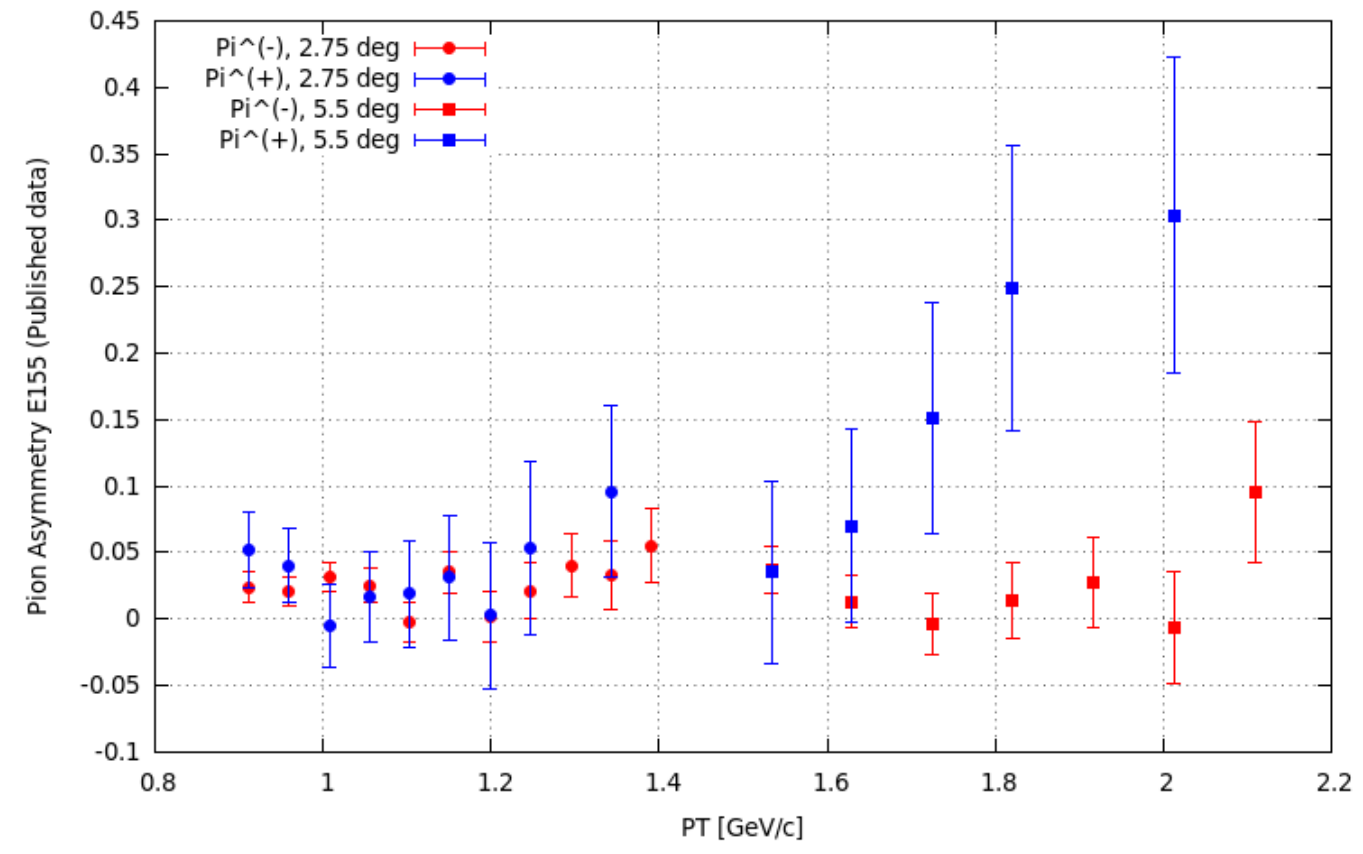

Figure 5.16

Plots of $\pi^{+}$(Blue) and $\pi^{-}$(Red) Asymmetries Versus $P_{T}$

In order to obtain the $\pi^{0}$ asymmetry from the $\pi^{+}$and $\pi^{-}$asymmetries, points in the same $P_{T}$ bins were averaged for all charges and spectrometer angles. In fitting the data, a simple constant fit of the form $a_{1}$ was preferred. Where $a_{1}$ is a constant to be determined from the fitting process.

The fit chosen is of the form

$$
A_{\|}=a_{1}
$$

The constant $a_{1}$ from the fitting process was found to be $a_{1}=0.0256 \pm 0.0032$ such that

$$
A_{\|}=0.0256 \pm 0.0032
$$

The data show good agreement with the fit especially at low $P_{T}$. Also, the following modifications were needed to be done namely: 


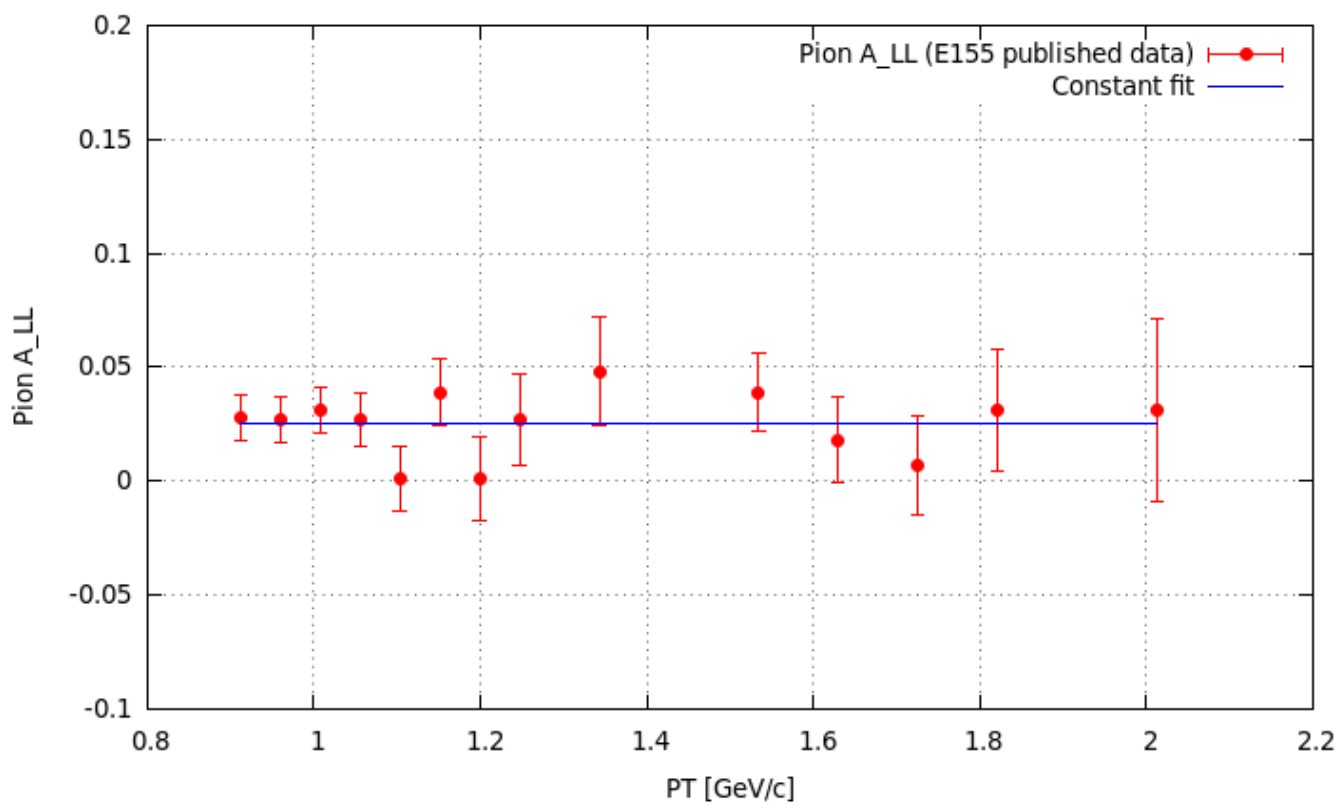

Figure 5.17

Parallel $\pi^{0}$ Asymmetry Versus $P_{T}$ with a Constant Fit (Blue Line).

1. $A_{\|}=A_{0^{\circ}}$ for SLAC, while for SANE $A_{\|}=A_{180^{\circ}}$. In order to appropriate and apply SLAC numbers suitable for SANE's usage a negative sign is added to $A_{0^{\circ}}$ since SANE measured $A_{180^{\circ}}$. Therefore, $A_{180^{\circ}}(\mathrm{SANE})=-A_{0^{\circ}}(\mathrm{SLAC})$.

2. The fit values need to be adjusted to account for the dilution of ${ }^{14} \mathrm{~N}$ for SANE and ${ }^{15} \mathrm{~N}$ for SLAC. The SANE experiment used ${ }^{14} \mathrm{NH}_{3}$ target while SLAC used ${ }^{15} \mathrm{NH}_{3}$. The dilution factor for SLAC, $f^{\prime}=0.13 \pm 0.03$. In order to calculate the background dilution for SANE, $f_{S A N E}$, careful but yet accurate estimation was done by considering the fact that: $f^{\prime}=0.13=3 /(3+15+x)$, where there are 3 protons, 15 nucleons in nitrogen, and $x$ is a constant to be determined such as: $x=\left[\mathrm{He}^{*}\left(1-p_{f}\right)+\mathrm{Al}\right.$ windows, etc] $/ p_{f}$, with $p_{f}$ being an unknown packing fraction. Solving for $x$ and using it to compute the corresponding value for $f_{S A N E}$ we arrived at $f_{S A N E}$ to be $0.14 \pm 0.032$.

Since there is little or no dependence of the SLAC $A_{\|}$pion on $P_{T}$, it would also be the case for SANE pion asymmetries as well. Furthermore, the fit values are adjusted 
by $0.13 / 0.14$ to account for the dilution of ${ }^{14} \mathrm{~N}$ (SANE) versus ${ }^{15} \mathrm{~N}$ (SLAC). Hence from equation (5.16), $A_{0^{\circ}}=0.024 \pm 0.003$.

Therefore, $A_{180^{\circ}}(\mathrm{SANE})=-0.024 \pm 0.003$. This value shows agreement with the SANE data $(-0.020 \pm 0.037)$ although SANE value has larger errors.

\subsection{2 $A_{b}$ for SANE's $A_{80^{\circ}}$ Configuration}

In the case for the perpendicular asymmetry, we proceed as in the previous subsection, however using the E155x SLAC data extracted from reference [87] (digitized from figures 24 and 25 [85]). The perpendicular $\pi^{0}$ asymmetry was obtained from a weighted average of the $\pi^{+}$and $\pi^{-}$asymmetries as shown in Figure 5.18.

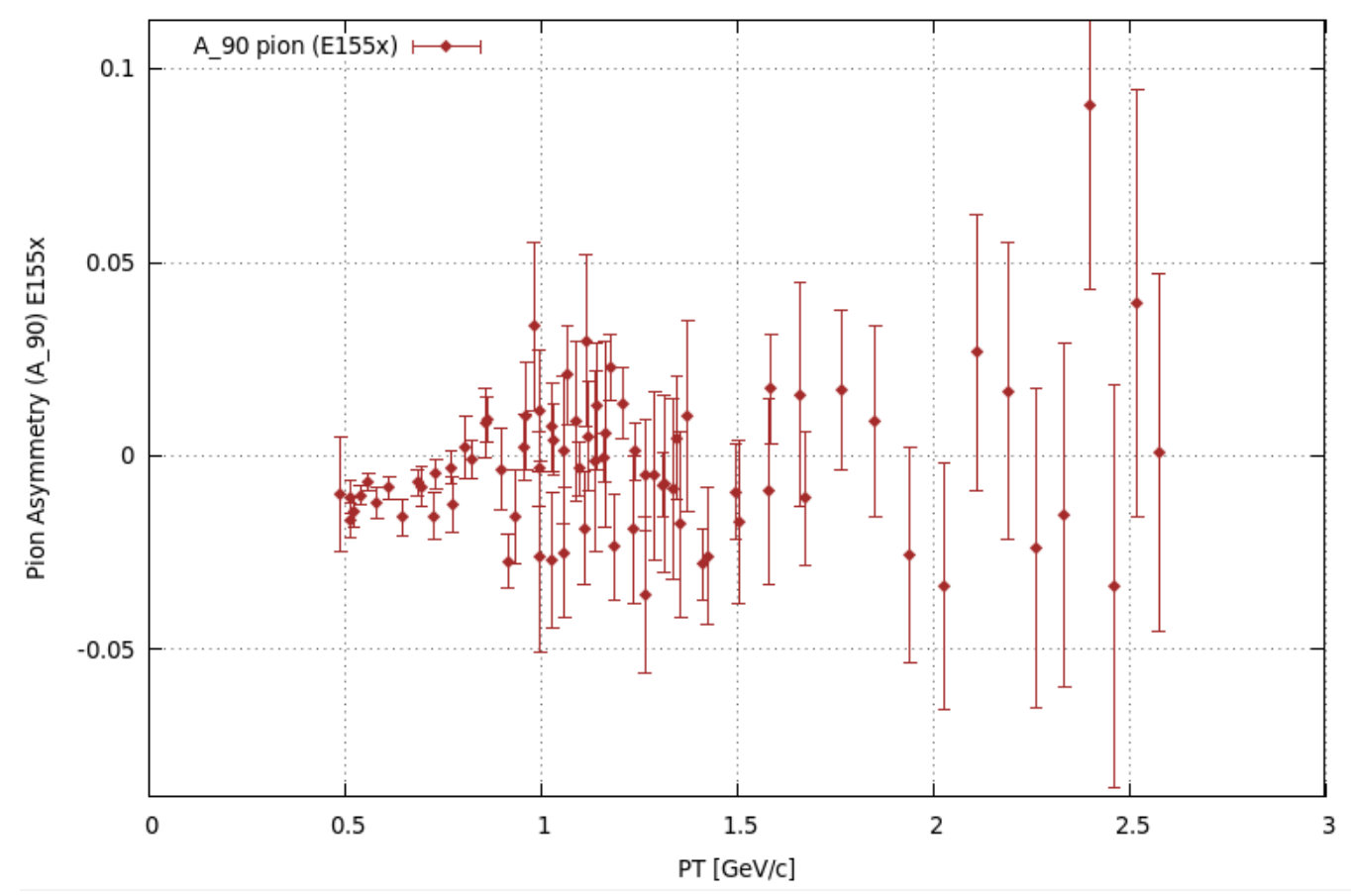

Figure 5.18

Perpendicular $\pi^{0}$ Asymmetry Versus $P_{T}$ 
A suitable fit to the data was found to be a constant fit such that only the data with $P_{T}>0.8 \mathrm{GeV} / \mathrm{c}$ which corresponds to about the range of pairs with $P_{T}>0.4 \mathrm{GeV} / \mathrm{c}$ for SANE [85].

Figure 5.18 shows a constant fit that was chosen to fit the data.

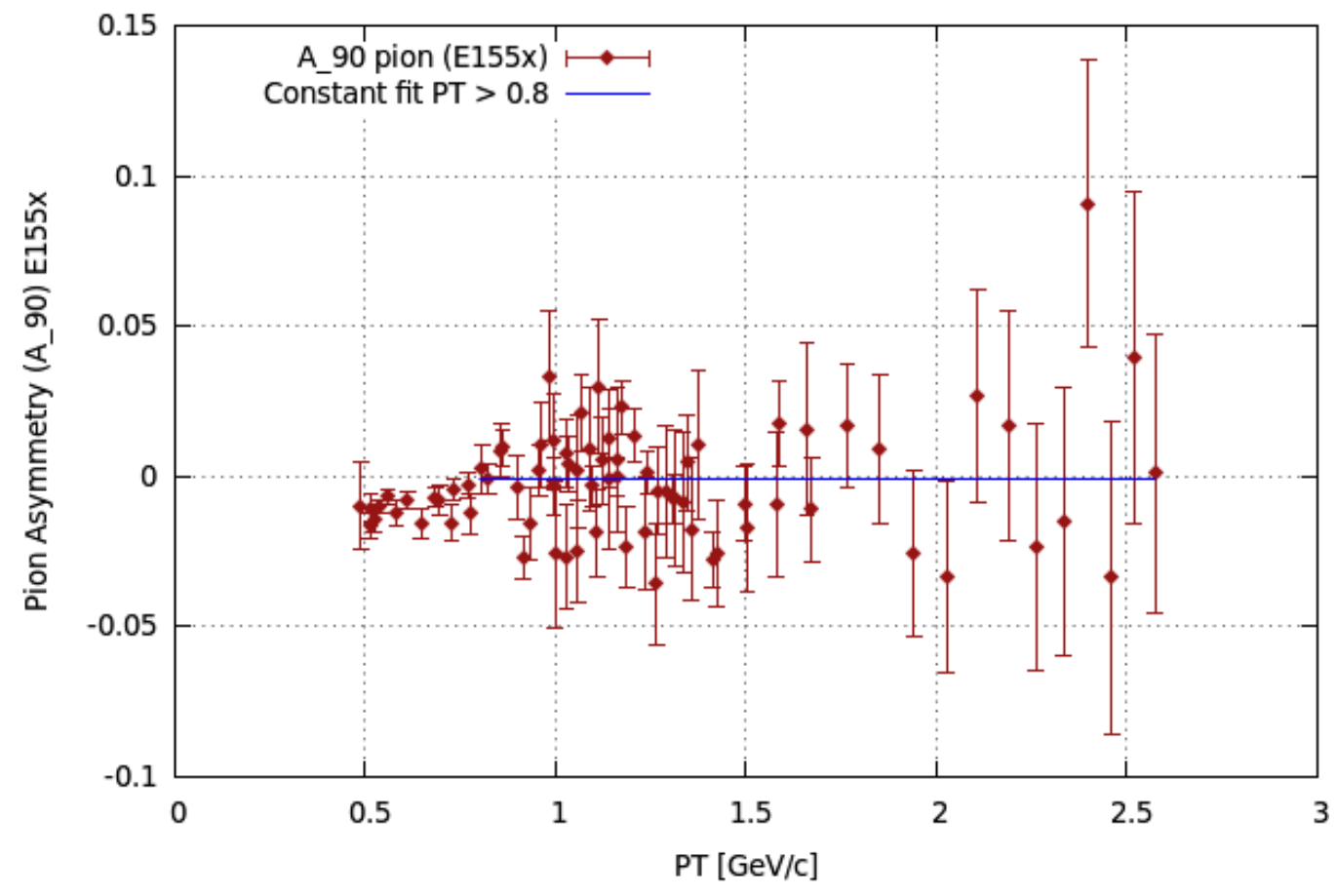

Figure 5.19

Fit to Perpendicular $\pi^{0}$ Asymmetry Data

The fit is of the form $A_{\perp}=a_{1}$ where $a_{1}=-0.00122 \pm 0.0016$. As before the fit values have to be adjusted by the dilution ratio of $0.13 / 0.14$.

$$
A_{\perp}=A_{92.4^{\circ}}=-0.00113 \pm 0.0015 .
$$


Because SLAC's data were collected at $92.4^{\circ}$, thus $A_{90}=\left[A_{92.4}-A_{0} \cos (92.4)\right] / \sin (92.4)=$ $-0.00013 \pm 0.0016$. Data in SANE were taken at $80^{\circ}$, therefore we used a linear combination of $A_{90}$ and $A_{180}$

$$
A_{80}=A_{90} \sin \left(80^{\circ}\right)+A_{0} \cos \left(80^{\circ}\right) .
$$

Even though $A_{90}=0$ for SANE, however, the error on $A_{90}$ for SLAC $( \pm 0.002)$ will be added to SANE's systematic errors. Therefore $A_{80}$ becomes:

$$
\begin{aligned}
A_{80} & =A_{90} \sin \left(80^{\circ}\right)+A_{0} \cos \left(80^{\circ}\right) \\
& =(0 \pm 0.002) * \sin \left(80^{\circ}\right)+(0.024 \pm 0.003) * \cos \left(80^{\circ}\right) \\
& =0.004 \pm 0.002 .
\end{aligned}
$$

The perpendicular pion asymmetry $A_{90^{\circ}}$ for SLAC is consistent with zero but with a non zero error. However, the $A_{80^{\circ}}$ for SANE is non zero with a non zero error.

\subsubsection{Calculating $f_{b}$}

The background dilution $f_{b}$ was calculated by simulating the ratio of background particles (positrons) to electrons. A fit to the simulated data resulted in a good estimation of $f_{b}$. A suitable form of such fit is given by:

$$
f\left(E^{\prime}\right)=a_{1} * e^{-a_{2} * E^{\prime}}
$$

where $E^{\prime}$ is the energy of the scattered particles, $a_{1}$ and $a_{2}$ are constants to be determined via the fitting process such that $a_{1}=0.938127 \pm 0.05938$ and $a_{2}=1.97656 \pm 0.06201$ Therefore

$$
f\left(E^{\prime}\right)=(0.938127 \pm 0.05938) * e^{-(1.97656 \pm 0.06201) * E^{\prime}}
$$




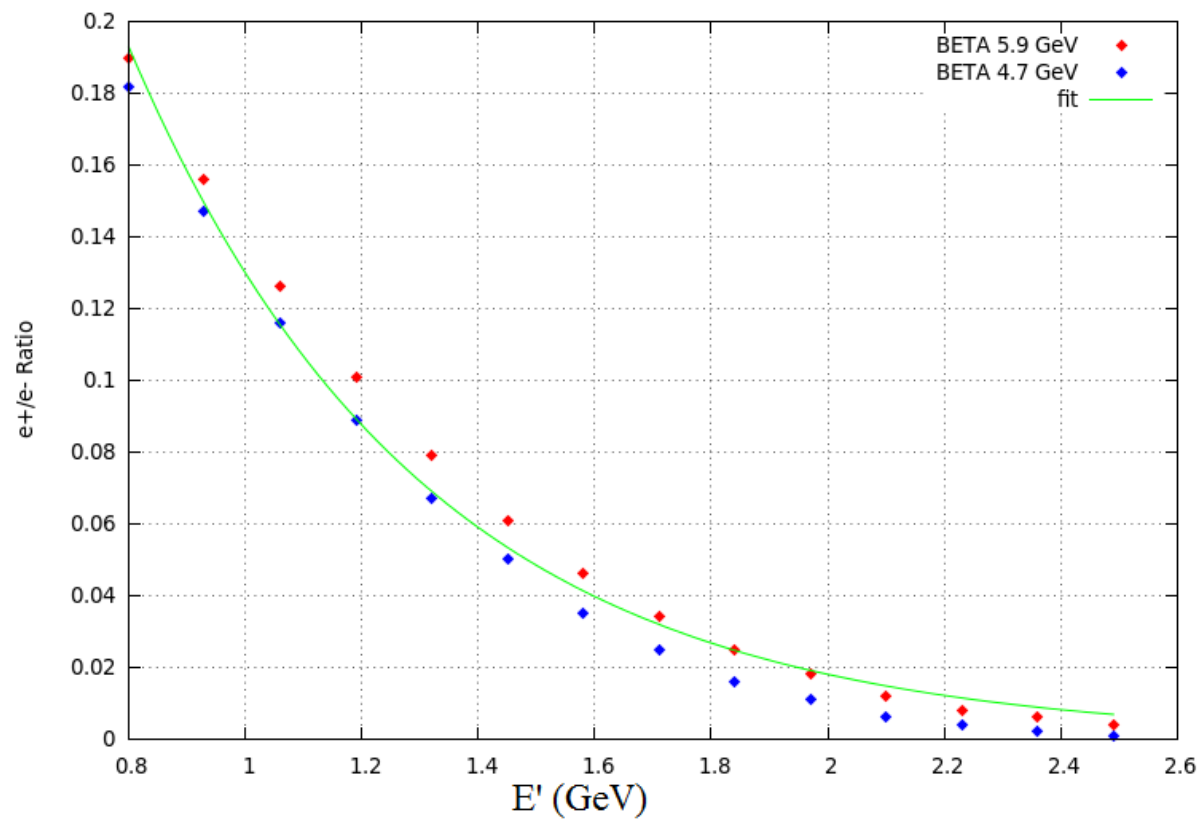

Figure 5.20

Ratio of Background Particles to Electrons at SANE Kinematics

Figure 5.20 shows the ratio of background particles to electrons plotted versus $E^{\prime}$. The red points represent when the beam energy is $5.9 \mathrm{GeV}$ while the blue points represent when the beam energy is $4.7 \mathrm{GeV}$. The green curve represent the fitting function. In the simulation BETA was set at a central angle of $40^{\circ}$.

Table 5.5 shows the connection between $f_{b}$ and $E^{\prime}$. At lower energies the background contribution is high therefore, in order to reduce pair-symmetric background it made sense to put a cut on the data if $f_{b}$ exceeded $10 \%$ which correspond to around $E^{\prime} \sim 1.3 \mathrm{GeV}$ as can be seen on Table 5.5.

Calculated values of the background corrections are presented in Table 5.6. It should be noted that equation (5.8) have been used to compute the numbers in the correction row 
Table 5.5

Ratio of Background Particles to Electrons

\begin{tabular}{cc}
\hline$E^{\prime}$ & $f_{b}$ \\
\hline 0.8 & 0.19 \\
0.93 & 0.156 \\
1.06 & 0.126 \\
1.19 & 0.101 \\
1.32 & 0.079 \\
1.45 & 0.061 \\
1.58 & 0.046 \\
1.71 & 0.034 \\
1.84 & 0.025 \\
1.97 & 0.018 \\
2.1 & 0.012 \\
2.23 & 0.008 \\
2.36 & 0.006 \\
2.49 & 0.004 \\
\hline
\end{tabular}

while equation (5.13) have been used to compute the systematic error row. The last row is a numerical example for $f_{b}=0.1, A_{m}\left(180^{\circ}\right)=0.36$ and $A_{m}\left(80^{\circ}\right)=0.019$. Also, the number in the table are only for $E^{\prime}$ such that $f_{b}\left(E^{\prime}\right)=0.1$, so $E^{\prime} \sim 1.3$. As $E^{\prime}$ increases, $f_{b}$ and the errors decrease, so that the table is a worst case.

Table 5.6 shows that both the correction and the systematic error on the final asymmetry $A$ (equation (5.8)) and its error (equation (5.13)) depend on the measured asymmetry $A_{m}$ and the background dilution $f_{b}$, for example when $f_{b}=0.1$, and $A_{m}\left(180^{\circ}\right)=0.36$, the $A_{180^{\circ}}$ corrected asymmetry and systematic error are written as:

$$
\begin{aligned}
A_{180} & =1.1 * A_{m}+0.003=0.406 \\
\frac{\delta A}{A} & =0.11 * \sqrt{\left(\frac{0.003}{1.1 * A_{m}+0.003}\right)^{2}+0.01}=1.1 \%
\end{aligned}
$$


Table 5.6

Error Results and the A Numerical Example of the Corrected Asymmetry $A$

\begin{tabular}{ccc}
\hline & $A_{180^{\circ}}$ & $A_{80^{\circ}}$ \\
\hline$A_{b} \pm \delta A_{b}$ & $-0.024 \pm 0.003$ & $0.004 \pm 0.002$ \\
$\delta f_{b} / f_{b}$ & 0.1 & 0.1 \\
Correction & $1.1 * A_{m}+0.003$ & $1.1 * A_{m}-0.0004$ \\
Systematic $\left(\frac{\delta A}{A}\right)$ & $0.11 * \sqrt{\left(\frac{0.003}{1.1 * A_{m}+0.003}\right)^{2}+0.01}$ & $0.11 * \sqrt{\left(\frac{0.002}{1.1 * A_{m}-0.0004}\right)^{2}+0.01}$ \\
Example $A$ & 0.406 & 0.021 \\
Example $\frac{\delta A}{A}$ & $1.1 \%$ & $1.5 \%$ \\
\hline
\end{tabular}

while for $A_{m}=0.019$, the $A_{80^{\circ}}$ asymmetry correction and systematic error are written as:

$$
\begin{aligned}
& A_{80}=1.1 * A_{m}-0.0004=0.021 \\
& \frac{\delta A}{A}=0.11 * \sqrt{\left(\frac{0.002}{1.1 * A_{m}-0.0004}\right)^{2}+0.01}=1.5 \% .
\end{aligned}
$$

Table 5.7 illustrates numerical cases for $A_{180^{\circ}}$ and $A_{80^{\circ}}$ with $A_{m}\left(180^{\circ}\right)=0.36$, $A_{m}\left(80^{\circ}\right)=0.019$ and different values of $f_{b}$.

It should be noted that the $10 \%$ relative dilution factor error was assumed as a reasonable estimate from the pair background simulations and comparison with data. Furthermore, the values for $A_{m}\left(180^{\circ}\right)$ and $A_{m}\left(80^{\circ}\right)$ are the preliminary results thus far. 
Table 5.7

Numerical Example to Illustrate the Corrected Asymmetries $A_{180^{\circ}}$ and $A_{80^{\circ}}$

\begin{tabular}{ccc}
\hline$f_{b}$ & $A_{180^{\circ}} \frac{\delta A}{A}$ & $A_{80^{\circ}} \frac{\delta A}{A}$ \\
\hline 0.1 & $0.406 \pm 1.1 \%$ & $0.021 \pm 1.5 \%$ \\
0.05 & $0.383 \pm 0.3 \%$ & $0.020 \pm 0.6 \%$ \\
0.004 & $0.365 \pm 0.004 \%$ & $0.019 \pm 0.042 \%$ \\
\hline
\end{tabular}




\section{CHAPTER 6}

\section{RESULTS}

In this chapter the results of the SANE analysis are presented. The selected events included in the analysis were divided into two category settings: anti-parallel target field $\left(180^{\circ}\right)$ and near perpendicular field setting $\left(80^{\circ}\right)$. These were further divided into two energy configurations, $4.7 \mathrm{GeV}$ and $5.9 \mathrm{GeV}$ that were in the kinematic region of $Q^{2}$ from 1.5 to $6.5 \mathrm{GeV}$ with $x$ ranging above 0.25 . Table 6.1 shows the SANE configuration.

Table 6.1

SANE Configuration

\begin{tabular}{cc}
\hline Energy & Orientation \\
\hline $5.9 \mathrm{GeV}$ & $80^{\circ}$ \\
$4.7 \mathrm{GeV}$ & $80^{\circ}$ \\
$5.9 \mathrm{GeV}$ & $180^{\circ}$ \\
$4.7 \mathrm{GeV}$ & $180^{\circ}$ \\
\hline
\end{tabular}

The results of the experimental physics asymmetries, $A_{180}$ and $A_{80}$ are presented in bins of $Q^{2}$ with respect to $x$ in section (6.1) followed by the results of the spin asymmetries $A_{1}$ and $A_{2}$ versus $W$ and $x$ in section (6.2). Furthermore, the results of the spin structure 
functions $g_{1}$ and $g_{2}$ are presented in section (6.3). Lastly, the background correction is presented in section (6.4).

\subsection{Results of $A_{180}$ and $A_{80}$}

Having applied all corrections, the experimental physics asymmetries are presented in Figure 6.1, Figure 6.2, Figure 6.3 and Figure 6.4, binned in $Q^{2}$ and $x$, and average by runs for the two target field configurations: anti-parallel $\left(180^{\circ}\right)$ and near perpendicular $\left(80^{\circ}\right)$ [56]. These are presented for the two energies used by SANE, 4.7 GeV and 5.9 GeV. The anti-parallel asymmetries are much larger than the near perpendicular values. The colored bands at the bottom of the plots represent the systematic error for the various $Q^{2}$ bins. From here we now proceeded in extracting the spin asymmetries and spin structure functions.

\subsection{Results of the Spin Asymmetries}

$A_{1}$ and $A_{2}$ were extracted from $A_{180}$ and $A_{80}$ as described in section (4.5). Plots of these results are shown in Figure 6.5, Figure 6.6 and Figure 6.7 [56]. SANE shows good

agreement with world data at large $W . A_{1}^{p}$ looks smooth versus $W . A_{2}^{p}$ is slightly zero at low and high $W$.

\subsection{Results of the Spin Structure Functions}

Shown in Figure 6.10 and Figure 6.9 are the results of $g_{1}$ and $g_{2}$ [56]. They where extracted as described in section (4.5). 


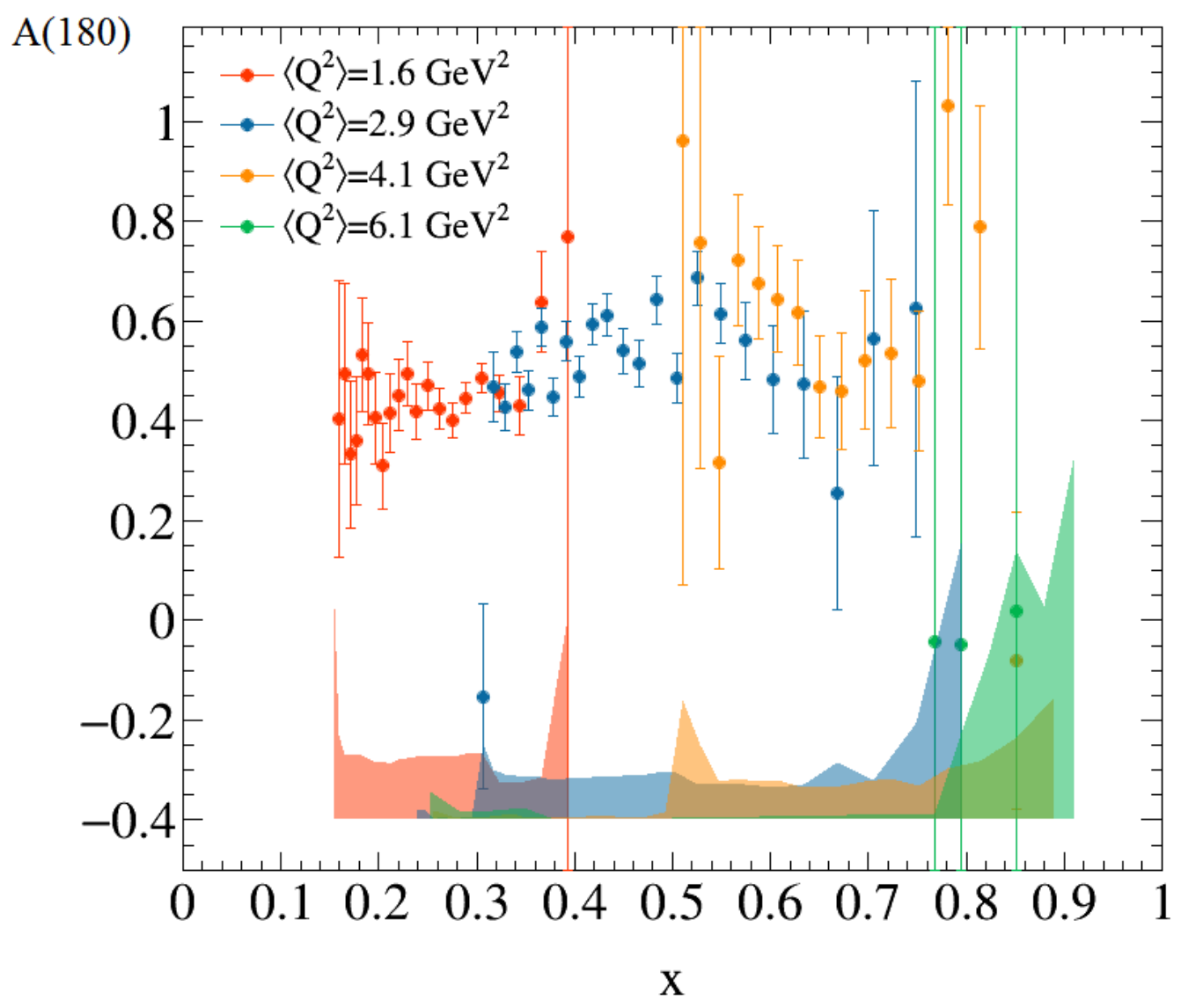

Figure 6.1

Plots of $A_{180}$ Versus $x$ at $4.7 \mathrm{GeV}$ 


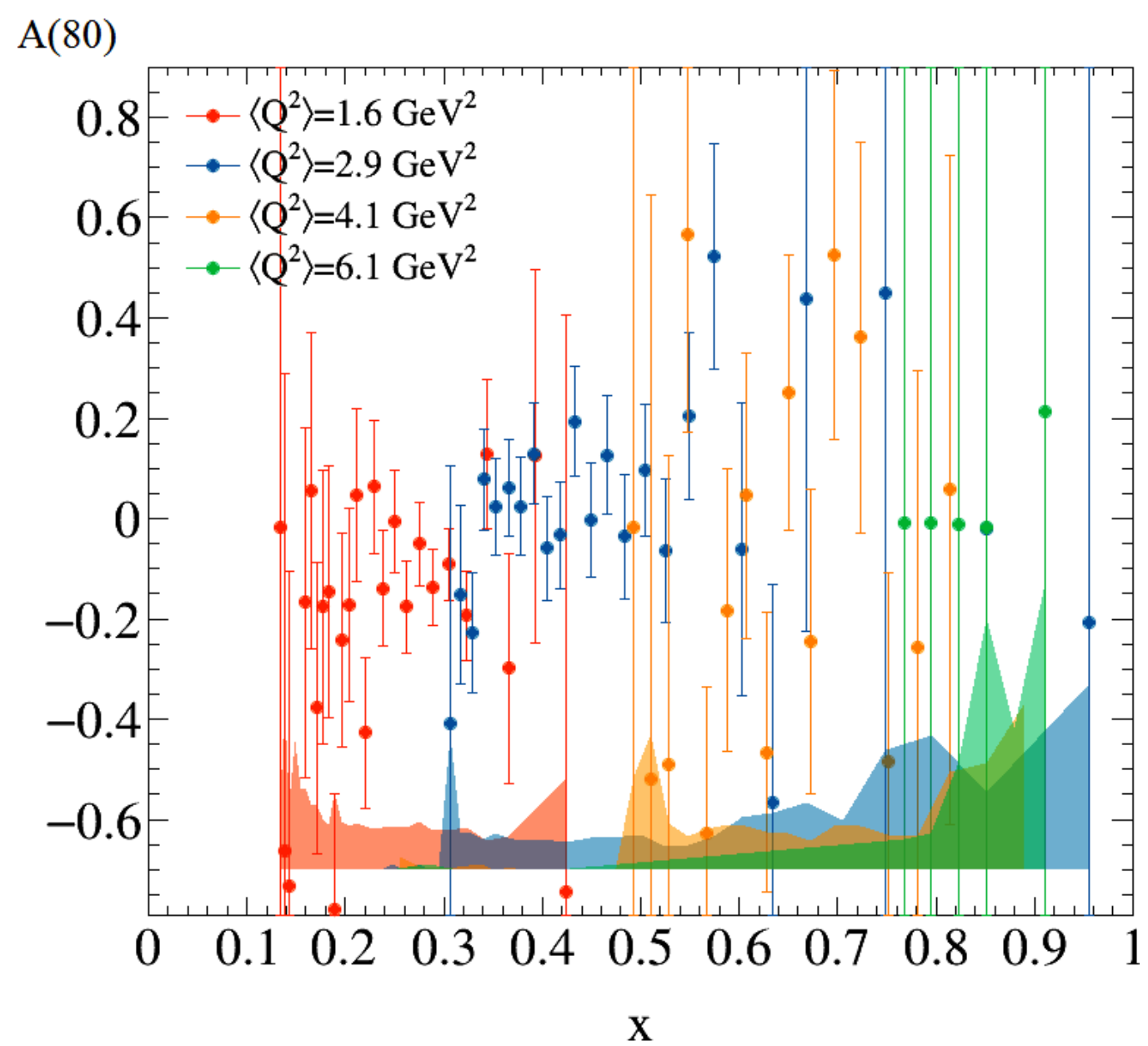

Figure 6.2

Plots of $A_{80}$ Versus $x$ at $4.7 \mathrm{GeV}$ 


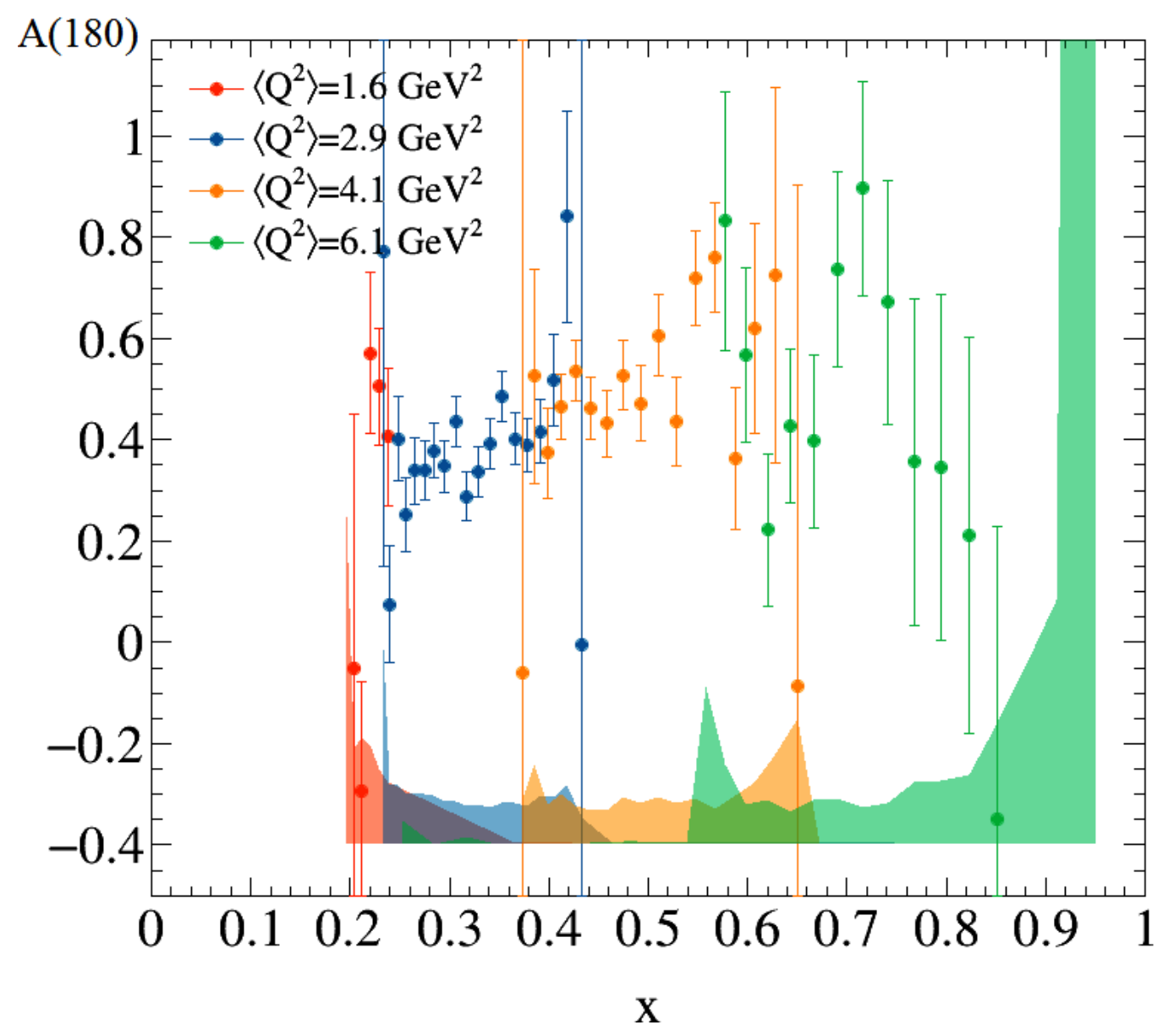

Figure 6.3

Plots of $A_{180}$ Versus $x$ at $5.9 \mathrm{GeV}$ 


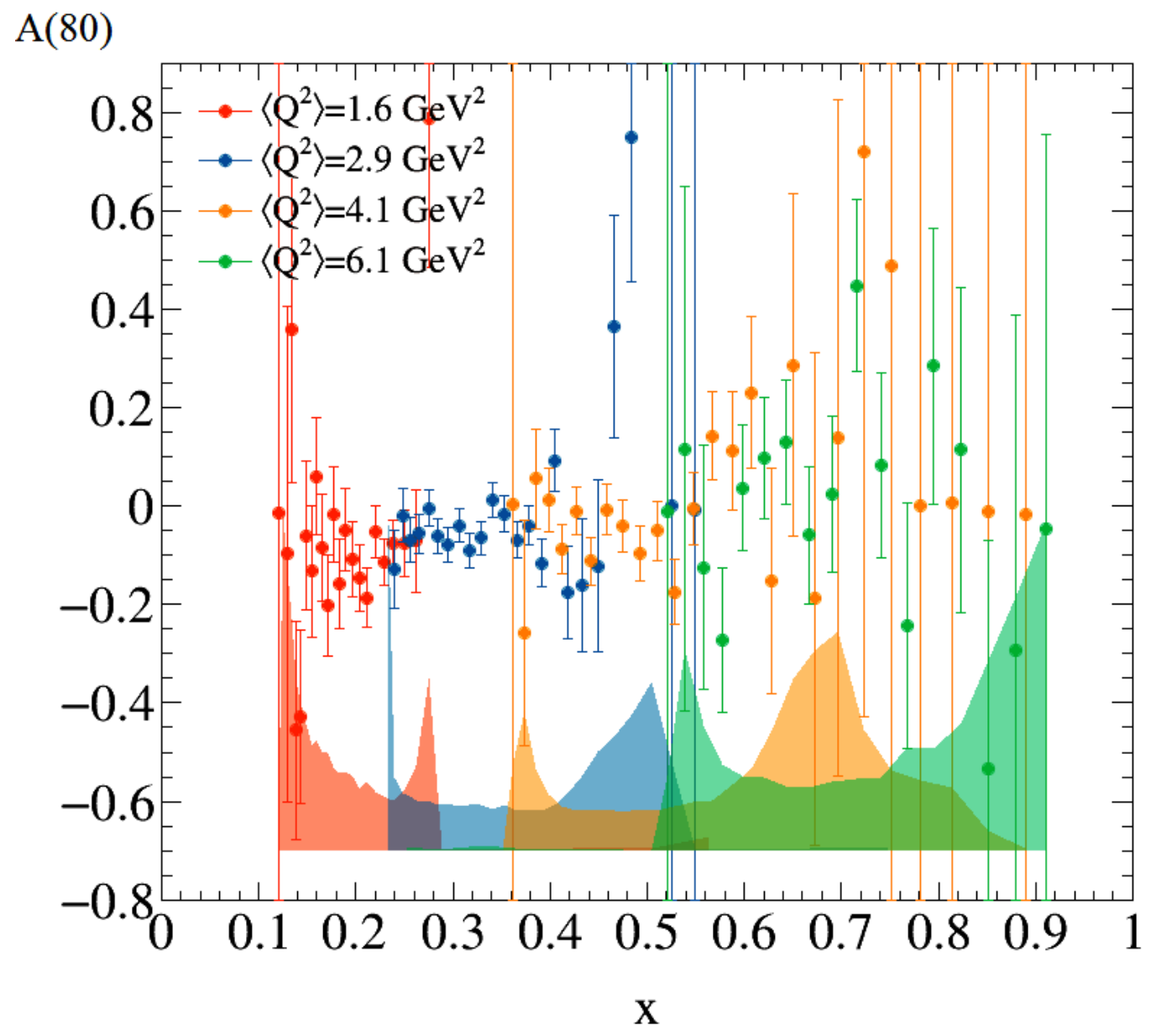

Figure 6.4

Plots of $A_{80}$ Versus $x$ at $5.9 \mathrm{GeV}$ 


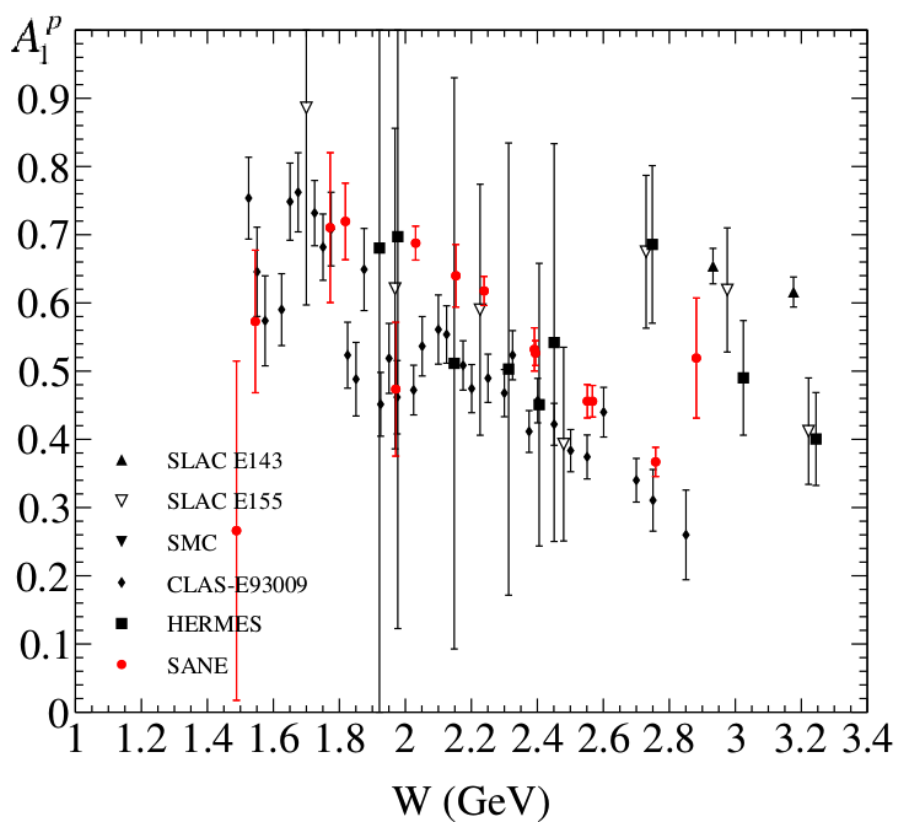

Figure 6.5

Plots of $A_{1}{ }^{p}$ Versus $W$

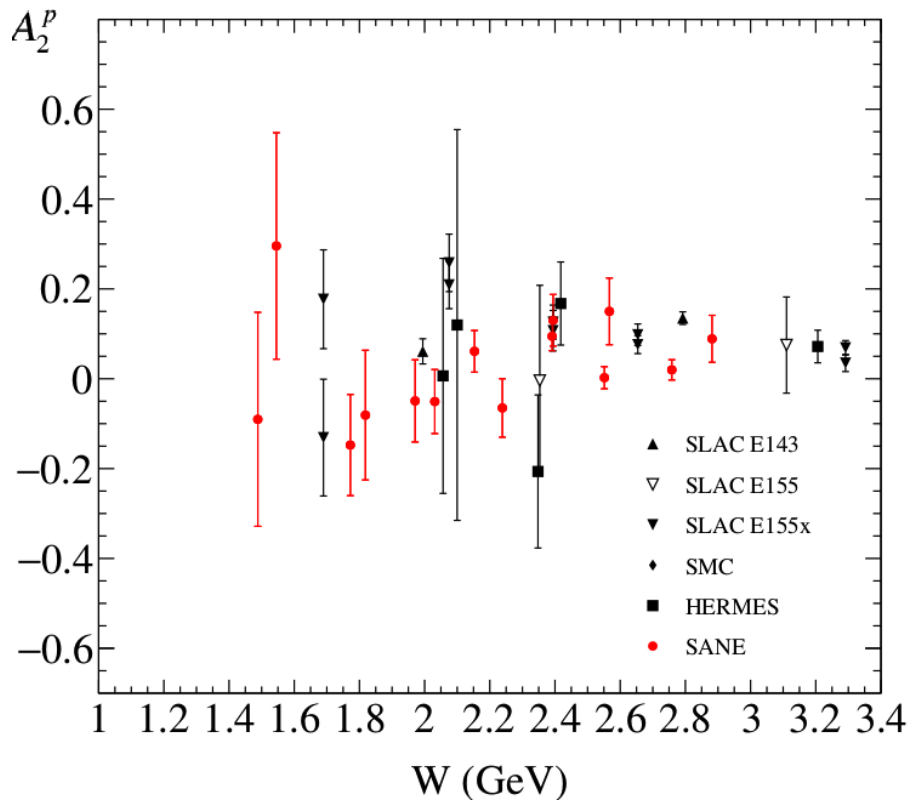

Figure 6.6

Plots of $A_{2}{ }^{p}$ Versus $W$ 


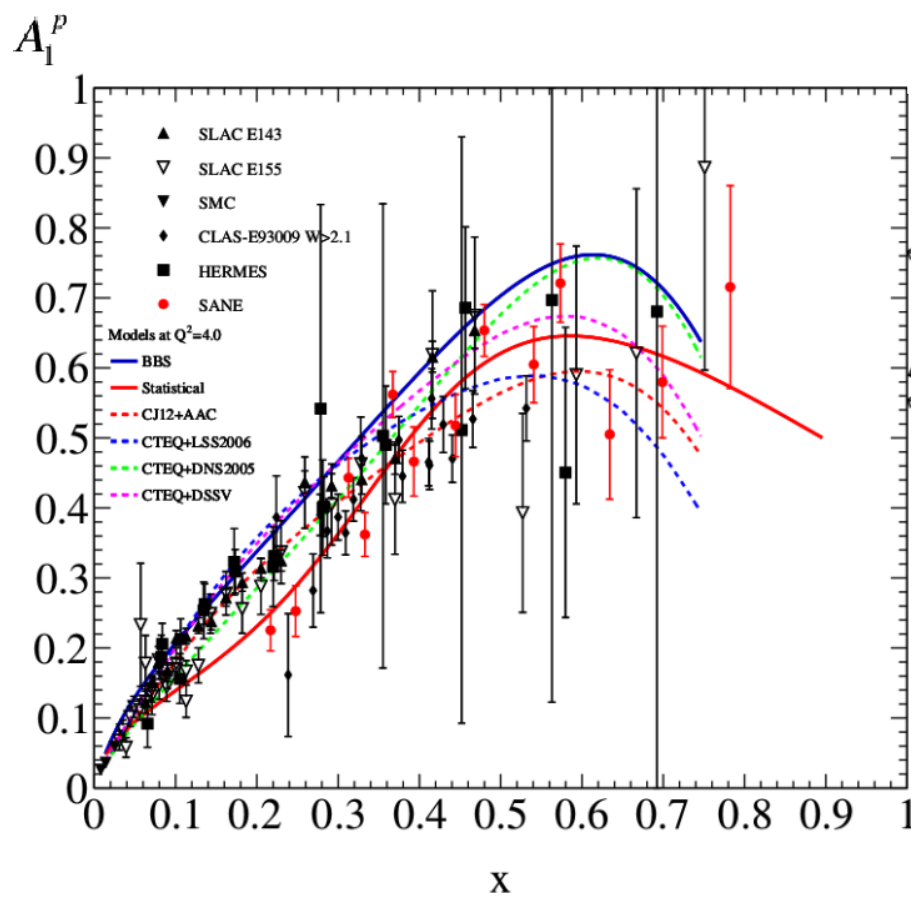

Figure 6.7

Plot of $A_{1}^{p}$ Versus $x$ 
As opposed to other experiments, SANE was a model independent experiment. Furthermore, SANE has more $g_{1}$ data than other experiments.

Figure 6.8 and Figure 6.9 show that $g_{1}$ has more data points with smaller statically error bars than $g_{2}$. Furthermore, at low $x$ we notice jumping points which are more pronounced in $g_{2}$, this is probably due to the effect of the background.

\subsection{Background Corrections}

SANE was an inclusive double spin asymmetry measurement by scattering longitudinally polarized electrons on a longitudinally and transversely polarized $\mathrm{NH}_{3}$ target. The measurements were done at momentum transfer of $1.5 \leq Q^{2} \leq 6.5 \mathrm{Ge} V^{2}$ and Bjorken $x$ of $0.25 \leq x \leq 0.8$. A significant background diluted our asymmetries particularly at low $E^{\prime}$ (low $x$ ). Photons from neutral pion decay convert to positron-electron pairs before reaching the Čerenkov. If these events pass the energy threshold, both electron and positron could be accepted in the calorimeter as good electron (DIS) events from primary scattering. A correction to this effect takes the form (as discussed in chapter 5)

$$
A=\frac{A_{m}-f_{b} A_{b}}{1-f_{b}}
$$

where $f_{b}$ is the background dilution, the ratio of the background count to all the polarized counts. Please refer to section 5.6 for a detail analysis and result. 


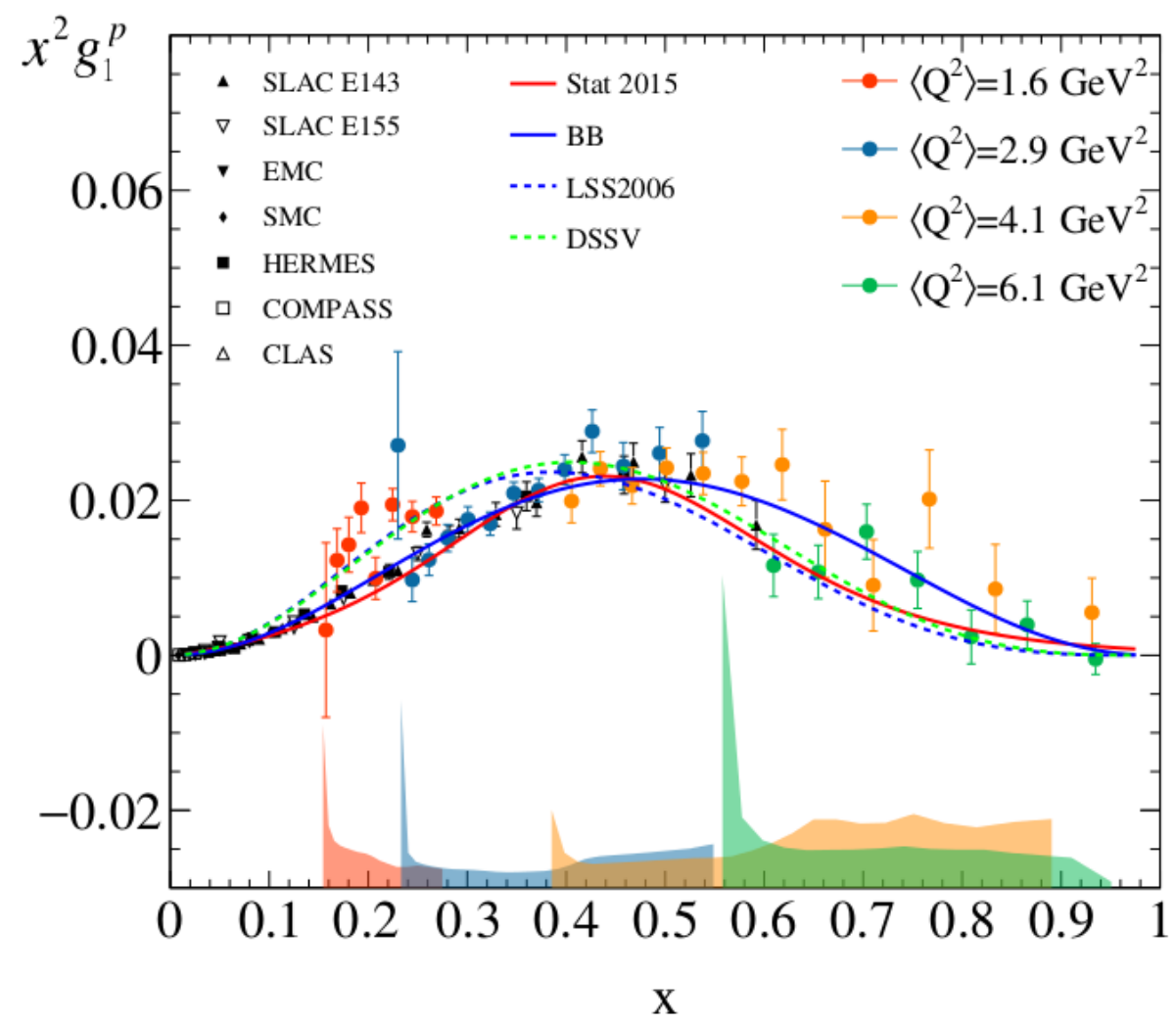

Figure 6.8

Results of $x^{2} g_{1}{ }^{p}$ Versus $x$ 


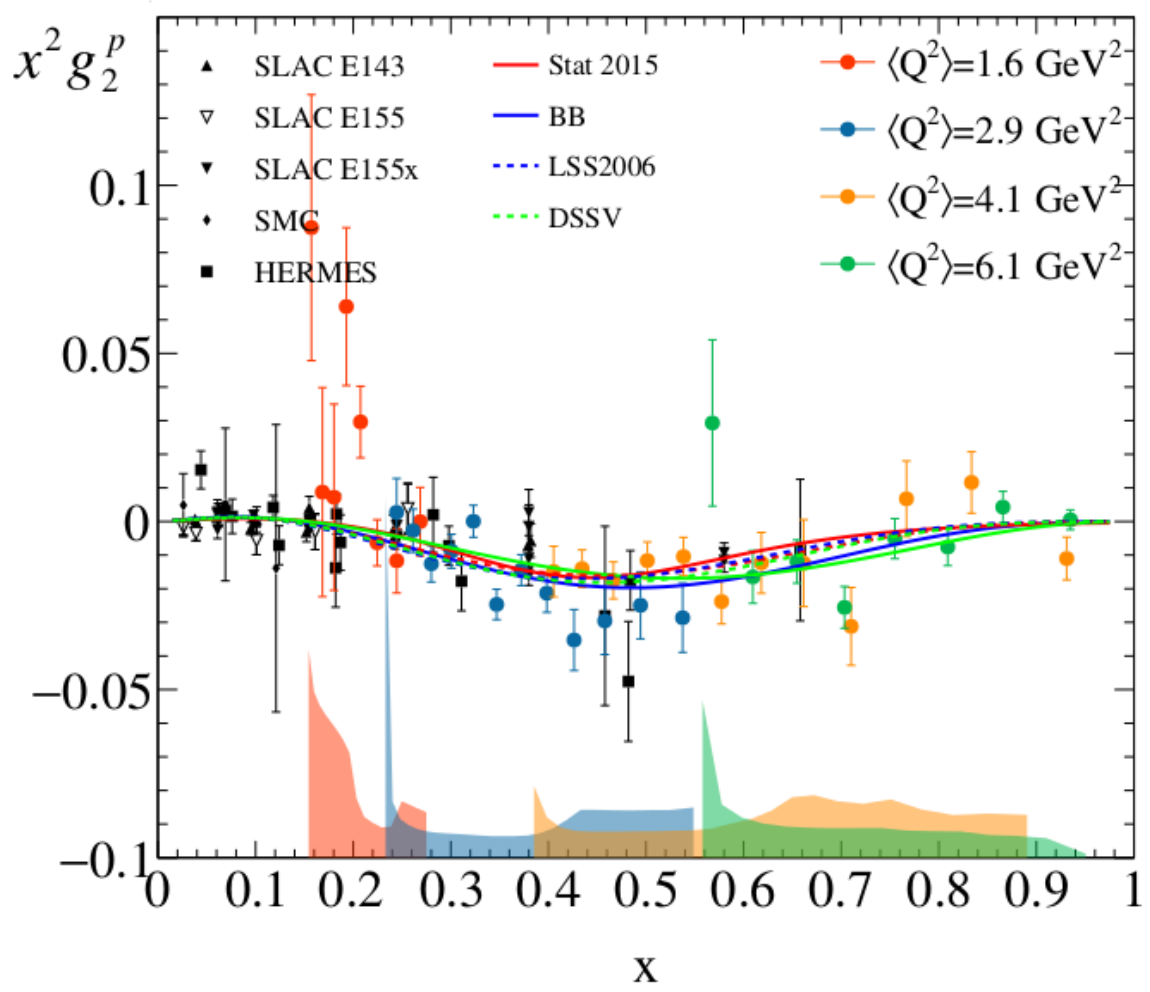

Figure 6.9

Results of $x^{2} g_{2}^{p}$ Versus $x$ 


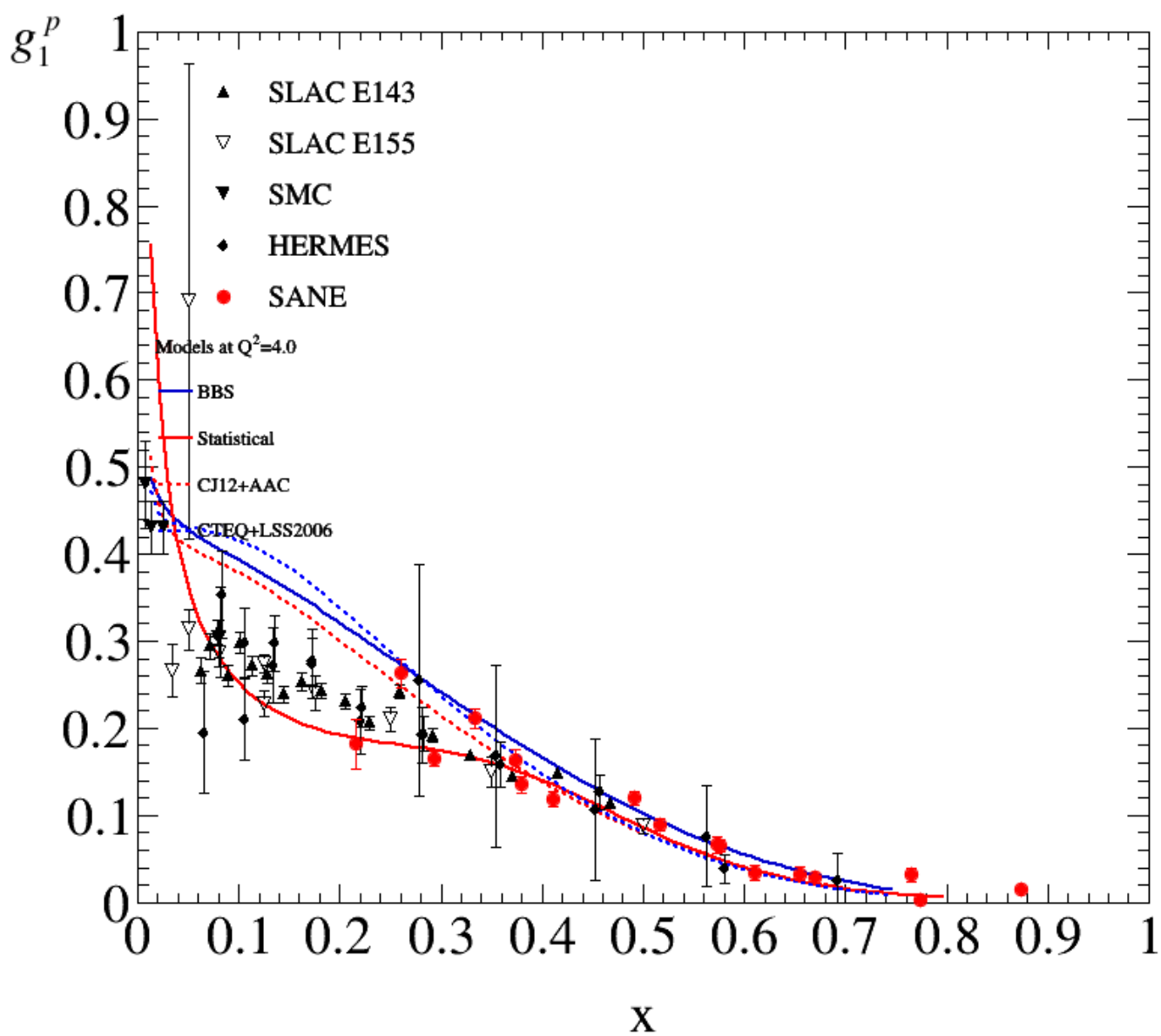

Figure 6.10

Results of $g_{1}{ }^{p}$ Versus $x$ 


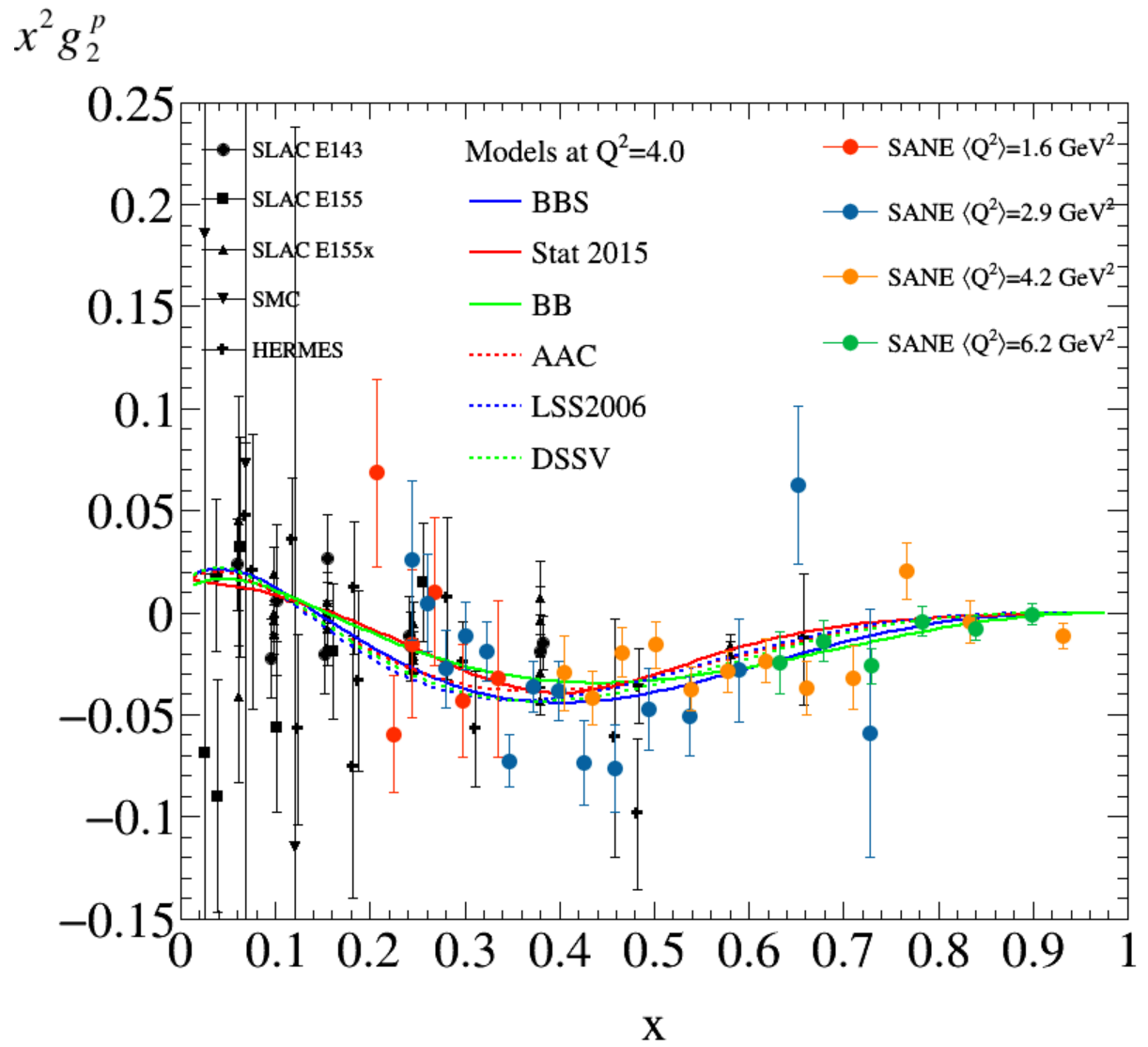

Figure 6.11

Results of $x^{2} g_{2}{ }^{p}$ Versus $x$ 


\section{CHAPTER 7}

\section{CONCLUSIONS}

\subsection{Conclusion}

Using the Continuous Electron Beam Accelerator Facility at Jefferson Laboratory, the Spin Asymmetries of the Nucleon Experiment in a model independent approach has produced valuable double polarization measurements of the proton's spin structure in the kinematic range of $x$ from 0.3 to 0.8 and $Q^{2}$ from 1.5 to $6.5 \mathrm{GeV}^{2}$. We have presented spin asymmetries $A_{1}$ and $A_{2}$ and the spin structure functions $g_{1}$ and $g_{2}$ in this region, as calculated from the data collected. With the inclusion of both anti-parallel and near perpendicular target orientation asymmetries, these calculations avoid the model dependence required by purely parallel datasets.

Being an inclusive electron scattering experiment, pair-symmetric backgrounds from neutral pion decays contributed significantly to measurements of the cross sections and asymmetries. Given that it was not possible to carry out measurements of the positively charged background using magnetic spectrometers as in other experiments, SANE made use of kinematic cuts to exclude data for which the ratio of background to signal would exceed $10 \%$, within a $\sim 20 \%$ relative uncertainty $[62,63]$. The results of the data analysis showed agreement with estimations from GEANT simulations. 
The importance of doing a pair symmetric background analysis can not be over emphasized. In order to get fully corrected measured asymmetries is the subtraction of the background pair symmetric and pion asymmetries. We have used SLAC data but suitably modified for the SANE to calculate the background asymmetries and background dilutions. In order to reduce these pair-symmetric background the data was cut if background dilution exceeded $10 \%$ which correspond to around $E^{\prime}>1.3 \mathrm{GeV}$.

These data offers a look at spin structure function $g_{2}$ with high accuracy. By both expanding the kinematic scope of existing measurements and contributing vastly to their statistical significance, SANE represents an important contribution to the understanding of nucleon spin structure an exciting expansion of nuclear physics. 


\section{REFERENCES}

[1] B. H. Bransden and C. J. Joachain Physics of Atom and Molecules. Addision Wesley Longman Limited, Edinburgh Gate, England, 1998.

[2] John T. Stock The Pathway to the Laws of Electrolysis Bull. Hist. Chem. 11 (1991).

[3] J.J. Thompson. Cathode rays. Philosophical Magazine, 1897.

[4] John Campbell Rutherford A Brief Biography Retrieved 4 March 2013.

[5] Romer A. Proton or prouton? Rutherford and the depths of the atom. Amer. J. Phys. 65 (8): 707, (1997).

[6] E. Rutherford. The scattering of $\alpha$ and $\beta$ particles by matter and the structure of the atom. Philosophical Magazine 21:669, 1911.

[7] H. Geiger and E. Marsden. The laws of deflexion of $\alpha$-particles through large angles. Philosophical Magazine 25:148, 1913.

[8] James Chadwick Possible Existence of a Neutron. Nature, p. 312, (Feb. 27, 1932).

[9] F. Halzen and A. Martin. Quarks and Leptons: An introductory course in modern particle physics. John Wiley and Sons, New York, USA, 1984.

[10] F. E. Close An Introduction to Quarks and Partons.

[11] Quang Ho-Kim, Xuan-Yem Pham Elementary particles and their interactions : concepts and phenomena.

[12] R.G. Roberts. Structure of the proton: deep inelastic scattering.

[13] HERMES Collaboration (A. Airapetian (Giessen U. and Michigan U.) et al.) Measurement of the virtual-photon asymmetry A2 and the spin-structure function $g 2$ of the proton. Eur.Phys.J. C72 (2012) 1921 (2012-03-13).

[14] P. Amaudruz et al. The ratio $F_{2}^{n} / F_{2}^{p}$ in deep inelastic muon scattering. Nuclear Physics B, 371(1-2):3 31, 1992.

[15] M. Arneodo et al. Reevaluation of the gottfried sum. Phys. Rev. D, 50:R1R3, Jul 1994. 
[16] J. Maxwell. Probing Proton Spin Structure: A measurement of $g_{2}$ at four momentum transfer of 2 to $6 \mathrm{GeV}^{2}$. PhD thesis, UVA, 2010.

[17] J. Mulholland. SANE's Measurement of the Proton's Virtual Photon Spin Asymmetry, $A_{1}^{p}$, at Large Bjorken $x$. PhD thesis, UVA, 2012.

[18] C. Yan. Slow raster system for polarized target experiments. Technical report, Jefferson Lab Hall C, 2008.

[19] [RSS Collaboration]. Proton spin structure in the resonance region, arXiv:nuclex/0608003.

[20] F. R. Wesselmann et al. Phys. Rev. Lett. 98, 132003, (30 March 2007).

[21] O. A. Rondon, RSS T. N. 2005-03 (2006).

[22] De Groot, N. and others, Measurement of the Spin Dependent Structure Functions of the Neutron and Proton, INSPIRE-1108855. 1996.

[23] P.M. King for the E155 and E155X collaboration Spin structure functions of the proton and deuteron from SLAC experiments E155 and E155X. SLAC-PUB-9741, 2002.

[24] Linda M. Stuart for the E143 collaboration Spin structure functions of the proton and deuteron from SLAC experiments E143. SLAC-PUB-7101, 1996.

[25] V.W. Hughes et al., Measurement of the Spin Dependent Structure Functions of the Neutron and Proton. CERN-SPSC-88-47 52p. (Dec 1988).

[26] D. Adams et al., Measurement of the Spin Dependent Structure Function $g_{1}(x)$ of the Proton. Phys.Lett. B329:399-406, 1994.

[27] Huang, J. et al. Beam-Target Double Spin Asymmetry $\left(A_{L} T\right)$ in Charged Pion Production from Deep Inelastic Scattering on a Transversely Polarized He-3 Target at $1.4<Q^{2}<2.7 \mathrm{GeV}^{2}$ Phys.Rev.Lett. 108 (2012) 052001.

[28] Donal Day. The Charge Form Factor of the Neutron, TJNAF E-93-026.

[29] Overhauser A. Phys. Rev. $92: 411$ (1953).

[30] Abragam A. and Proctor WJ. Compt. Rend. Acad. Sci. 246:2253 (1958).

[31] Jeffries CD. Phys. Rev. 117:1056 (1960).

[32] SANE Collaboration. www.hallcweb.jlab.org/experiments/sane/rondon/chicane.pdf Chicane setting analysis page.

[33] David Griffiths Introduction to Elementary Particles. WILEY-VCH Verlag GmbH and Co. KGaA, Weinheim 2004. 
[34] J. Beringer et al (Particle Data Group), Phys. Rev., D86, 010001 (2012).

[35] W. Wandzura and F. Wilczek, Phys.Lett. B172 (1977) 195.

[36] SANE Collaboration Update to TJNAF E03-007; Spin Asymmetries of the Nucleon Experiment 2006.

[37] Philip G. Ratcliffe $Q C D$ and Transverse-Spin Physics. arXiv:hep-ph/0310150v1 2003.

[38] A. Airapetian et al. [HERMES Collaboration], Phys. Rev. Lett. 92, 012005 (2004).

[39] K. V. Dharmawardane et al. [CLAS Collaboration], Phys. Lett. B 641, 11 (2006).

[40] X. Zheng et al. [Jefferson Lab Hall A Collaboration], Phys. Rev. Lett. 92, 012004 (2004).

[41] R. Van de Vyver [A2/GDH Collaboration], Nul. Phys. A689, 379 (2001).

[42] K. Helbing [GDH Collaboration], Status of the GDH experiment at ELSA”, Published in Mainz 2000, Gerasimov-Drell-Hearn sum rule and the nuleon spin struture in the resonane region, p. 133.

[43] S. Brodsky, M. Burkardt, and I. Shmidt, Nul. Phys. B441, 197 (1995).

[44] Xiangdong Ji Spin Structure Functions of the Nucleon arXiv:hep-ph/9510362. 1995.

[45] C. Amsler et al., Physics Letters B667, 1 (2008).

[46] Friedmann, Kendall and Taylor et al Deep Inelastic Scattering, Oxford University, Physics Department, 2003.

[47] C. K. Sinclair Technical Notes: Beam Energy Combination which Provide Simultaneous Longitudinal Electron Polarization in Two Experimental Halls.

[48] RSS Collaboration Proton spin structure in the resonance region arXiv:nuclex/0608003.

[49] Donal Day The Charge Form Factor of the Neutron, TJNAF E-93-026

[50] Overhauser A. Phys. Rev. 92:411 (1953)

[51] Abragam A Proctor WJ. Compt. Rend. Acad. Sci. 246:2253 (1958)

[52] Jeffries CD. Phys. Rev. 117:1056 (1960)

[53] D.G. Crabb and W. Meyer. Solid polarized targets for nuclear and particle physics experiments. Annual Review of Nuclear and Particle Science, 47:67109, 1997.

[54] J.D. Jackson, S.B. Treiman, and H.W. Wyld, Jr., Phys. Rev. 106, 517 (1957). 
[55] D.H. Wilkinson, Nucl. Phys. A377, 474 (1982).

[56] W. Armstrong. Measurement of the Proton $A_{1}$ and $A_{2}$ Spin Asymmetries: Probing Color Forces. PhD thesis, Temple University, 2015.

[57] SLAC E80, M. J. Alguard et al., Phys. Rev. Lett. 37, 1261 (1976); 41, 70 (1978).

[58] SLAC E130, G. Baum et al., Phys. Rev. Lett. 51, 1135 (1983).

[59] O. A. Rondon, The Packing Fraction and Dilution Factor in RSS, RSS T. N. 2005-03 (2006).

[60] G. Warren, GEANT Simulation and Analysis Package of BETA. (2003).

[61] K. V. Dharmawardane, et al. [CLAS Collaboration], Phys. Lett. B641, 11 (2007).

[62] K. V. Dharmawardane, Report On Pair Symmetric Background. SANE meeting VII. (2005).

[63] O. A. Rondon, SANE Pair-Symmetric Background Run Plan. SANE Work Meeting. (2007).

[64] J.D. Bjorken, Inelastic Scattering of Polarized Leptons from Polarized Nucleons Phys. Rev. D 1 (1970) 1376.

[65] C.S. Armstrong et al. Moments of the Proton $F_{2}$ Structure Function at Low $Q^{2}$. Phys. Rev. D63:094008, 2001.

[66] J. Maxwell. Polarized target analysis for the spin asymmetries of the nucleon experiment. SANE technical report, April 2010.

[67] V. Breton, H. Fonvieille, P. Grenier, C. Guicheney, J. Jousset, Y. Roblin, and F. Tamin. Application of neural networks and cellular automata to interpretation of calorimeter data. Nuclear Instruments and Methods in Physics Research Section A: Accelerators, Spectrometers, Detectors and Associated Equipment, 362(2-3):478 486, 1995.

[68] K. Slifer Radiation Lengths used in E94010 Analysis, December 10, 2002.

[69] James Maxwell Elastic Radiative Tail Correction in SANE, April 27, 2011.

[70] J. Mulholland Getting the Structure functions from Measurable Asymmetries SANE Tech note June 6, 2011.

[71] O. A. Rondon Simplified Expressions to get $A_{1}$ and $A_{2}$ from $A_{8} 0$ and $A_{1} 80$ in SANE.

[72] L. W. MO and Y. S. TSAI. Radiative corrections to elastic and inelastic ep and up scattering. Rev. Mod. Phys., 41(1):205 235, Jan 1969. 
[73] D. Gaskell and J. Maxwell. https://hallcweb.jlab.org/experiments/sane/ wiki/index.php/Moller_Polarimeter.

[74] O. A. Rondon https://userweb.jlab.org/ rondon/analysis/asym/df/pfs-final-v3.pdf.

[75] Hovhannes Baghdasaryan. Pair-Symmetric Background Asymmetry. Private communication, 2011.

[76] [SANE Collaboration], Spin Asymmetries on the Nucleon Experiment (SANE). (April 28, 2003).

[77] J.O'Connell Predicting Inclusive Electropion and Nucleon Cross Sections for High Particle Momenta. National Burean of Standards, p. 345-349, 1988.

[78] David E. Wiser, Inclusive Photoproduction of Protons, Kaons, and Pions at SLAC Energies 1977.

[79] O. A. Rondon Inclusive Pion and Nucleon Electroproduction https://hallcweb.jlab.org/experiments/sane/wiki/index.php/ Inclusive_pion_and_nucleon_electroproduction.

[80] Hovhannes Baghdasaryan Data to MC pion rate comparison https://hallcweb.jlab.org/experiments/sane/wiki/index.php/ Data_to_MC_pion_rate_comparison.

[81] Whitney R. Armstrong. Inclusive Pion Production Models 2014.

[82] J.W. Jr. Lightbody and J.S. O'Connell. Modeling single arm electron scattering and nucleon production from nuclei by Gev electrons. Computers in Physics, 2:57-64, 1988.

[83] Narbe kalantarians Update on Dilution Factors, SANE Collaboration Meeting, February 2012.

[84] P.L. Anthony et al. [E155 Collaboration] Inclusive Hadron Photoproduction from Longitudinally Polarized Protons and Deuterons. Phys. Lett. B458, 536 (1999), [hep$\mathrm{ph} / 9902412]$.

[85] O. A. Rondon Fits to charged pion asymmetries https://hallcweb.jlab.org/experiments/sane/wiki/ index.php/Fits_to_charged_pion_asymmetries.

[86] M. Perl, High Energy Hadron Physics, John Wiley and Sons Inc (December 4, 1974), New York.

[87] Nawal Benmouna, A Precise Measurement of the Spin Structure Functions g2p 2001.

[88] Steven D. Bass The Spin Structure of the Proton. World Scientific Publishing Co. Pte. Ltd. 5 Toh Tuck Link, Singapore 5962242008. 
APPENDIX A

BEAM ENERGY COMBINATIONS FOR EXPERIMENTAL HALLS AND $\pi^{0}$ ASYMMETRY DATA FOR ALL RUNS 


\section{A.1 Beam Energy Combinations for Experimental Halls}

Here, we give the 21 values of the precession difference function, $f$ for the cases of halls $\mathrm{A}$ and $\mathrm{B}$, and for halls $\mathrm{C}$ and $\mathrm{B}$, as well as for halls $\mathrm{A}$ and $\mathrm{C}$. Each three line entry in the tables gives, in addition to the value of the function $f\left(n_{\sigma}, n_{\tau}\right)$, the range of values of $m_{\sigma}-m_{\tau}$ which can be obtained by operation of the accelerator with five pass energies between 2 and $6 \mathrm{GeV}$, and the total number of such values over this energy range.

For each particular choice of two halls, $\sigma$ and $\tau$, the general form of the precession difference equation is:

$$
\frac{\theta_{\sigma}-\theta_{\tau}}{\pi}=\operatorname{Pf}\left(n_{\sigma}, n_{\tau}\right)=m_{\sigma}-m_{\tau}
$$

To illustrate the use of these tables to find the beam energy combination which provide simultaneous longitudinal polarization in two particular halls, consider the case of providing 3 pass beam to hall $\mathrm{A}$ and 5 pass beam to hall $\mathrm{C}$, with a five pass energy close to $4 \mathrm{GeV}$.

From the table for halls A and C, we obtain the value of $f\left(n_{A}=3, n_{C}=5\right)=-27.069792$. Allowing values for $\left(m_{A}-m_{C}\right)$ to range between -13 amd -36 . Since we want an energy close to the midrange of the allowed values, e.g., -24 or -25 , will provide the best choice. Working through the numbers for these two cases, we find that $m_{A}-m_{C}=-24$ corresponds to a linac energy of $390.6778 \mathrm{MeV}$, and $m_{A}-m_{C}=-25$ corresponds to 406.9560 $\mathrm{MeV}$. These values for the linac energy give five pass energies of $3.9507 \mathrm{GeV}$ and 4.1153 $\mathrm{GeV}$, respectively. For the case with the linac energy of $390.6778 \mathrm{MeV}$, we compute a total precession of $14.926694 \pi$ to hall A. Thus, to obtain the longitudinal polarization in hall 
A, we need to add $0.073306 \pi=13.195^{\circ}$ to the polarization direction at the injector, in the horizontal plane.

\begin{tabular}{|c|c|c|c|c|c|}
\hline & $\mathrm{n}_{\mathrm{A}}=1$ & $\mathrm{n}_{\mathrm{A}}=2$ & $\mathrm{n}_{\mathrm{A}}=3$ & $\mathrm{n}_{\mathrm{A}}=4$ & $\mathrm{n}_{\mathrm{A}}=5$ \\
\hline $\mathrm{n}_{\mathrm{B}}=1$ & $\begin{array}{l}\text { Condition } \\
\text { Not } \\
\text { Allowed }\end{array}$ & $\begin{array}{l}6.081771 \\
3 \text { to } 8 \\
6 \text { values }\end{array}$ & $\begin{array}{l}15.723438 \\
8 \text { to } 21 \\
14 \text { values }\end{array}$ & $\begin{array}{l}29.365104 \\
14 \text { to } 39 \\
26 \text { values }\end{array}$ & $\begin{array}{l}47.006771 \\
22 \text { to } 64 \\
43 \text { values }\end{array}$ \\
\hline $\mathrm{n}_{\mathrm{B}}=2$ & $\begin{array}{l}-4.784896 \\
-3 \text { to }-6 \\
4 \text { values }\end{array}$ & $\begin{array}{l}\text { Condition } \\
\text { Not } \\
\text { Allowed }\end{array}$ & $\begin{array}{l}10.498438 \\
5 \text { to } 14 \\
10 \text { values }\end{array}$ & $\begin{array}{l}24.140104 \\
11 \text { to } 32 \\
22 \text { values }\end{array}$ & $\begin{array}{l}41.781771 \\
19 \text { to } 56 \\
38 \text { values }\end{array}$ \\
\hline $\mathrm{n}_{\mathrm{B}}=3$ & $\begin{array}{l}-14.009896 \\
-7 \text { to }-19 \\
13 \text { values }\end{array}$ & $\begin{array}{l}-8.368229 \\
-4 \text { to }-11 \\
8 \text { values }\end{array}$ & $\begin{array}{l}\text { Condition } \\
\text { Not } \\
\text { Allowed }\end{array}$ & $\begin{array}{l}14.915104 \\
7 \text { to } 20 \\
14 \text { values }\end{array}$ & $\begin{array}{l}32.556771 \\
15 \text { to } 44 \\
30 \text { values }\end{array}$ \\
\hline $\mathrm{n}_{\mathrm{B}}=4$ & $\begin{array}{l}-27.234896 \\
-13 \text { to }-37 \\
25 \text { values }\end{array}$ & $\begin{array}{l}-21.593229 \\
-10 \text { to }-29 \\
20 \text { values }\end{array}$ & $\begin{array}{l}-11.951563 \\
-6 \text { to }-16 \\
11 \text { values }\end{array}$ & $\begin{array}{l}\text { Condition } \\
\text { Not } \\
\text { Allowed }\end{array}$ & $\begin{array}{l}19.331771 \\
9 \text { to } 26 \\
18 \text { values }\end{array}$ \\
\hline $\mathrm{n}_{\mathrm{B}}=5$ & $\begin{array}{l}-44.459896 \\
-21 \text { to }-60 \\
40 \text { values }\end{array}$ & $\begin{array}{l}-38.818229 \\
-18 \text { to }-52 \\
35 \text { values }\end{array}$ & $\begin{array}{l}-29.176563 \\
-14 \text { to }-39 \\
26 \text { values }\end{array}$ & $\begin{array}{l}-15.534896 \\
-8 \text { to }-21 \\
14 \text { values }\end{array}$ & $\begin{array}{l}2.106771 \\
1 \text { to } 2 \\
2 \text { values }\end{array}$ \\
\hline
\end{tabular}

Figure A.1

Precession Difference Functions for Halls A and B 


\begin{tabular}{|c|c|c|c|c|c|}
\hline & $\mathrm{n}_{\mathrm{c}}=1$ & $\mathrm{n}_{\mathrm{c}}=2$ & $\mathrm{n}_{\mathrm{c}}=3$ & $\mathrm{n}_{\mathrm{c}}=4$ & $\mathrm{n}_{\mathrm{C}}=5$ \\
\hline $\mathrm{n}_{\mathrm{B}}=1$ & $\begin{array}{l}\text { Condition } \\
\text { Not } \\
\text { Allowed }\end{array}$ & $\begin{array}{l}4.368229 \\
2 \text { to } 5 \\
4 \text { values }\end{array}$ & $\begin{array}{l}13.176563 \\
6 \text { to } 17 \\
12 \text { values }\end{array}$ & $\begin{array}{l}25.984896 \\
12 \text { to } 35 \\
24 \text { values }\end{array}$ & $\begin{array}{l}42.793229 \\
20 \text { to } 58 \\
39 \text { values }\end{array}$ \\
\hline $\mathrm{n}_{\mathrm{B}}=2$ & $\begin{array}{l}-5.665104 \\
-3 \text { to }-7 \\
5 \text { values }\end{array}$ & $\begin{array}{l}\text { Condition } \\
\text { Not } \\
\text { Allowed }\end{array}$ & $\begin{array}{l}7.951563 \\
4 \text { to } 10 \\
7 \text { values }\end{array}$ & $\begin{array}{l}20.759896 \\
10 \text { to } 28 \\
19 \text { values }\end{array}$ & $\begin{array}{l}37.568299 \\
18 \text { to } 51 \\
34 \text { values }\end{array}$ \\
\hline $\mathrm{n}_{\mathrm{B}}=3$ & $\begin{array}{l}-14.890104 \\
-7 \text { to }-20 \\
14 \text { values }\end{array}$ & $\begin{array}{l}-10.081771 \\
-5 \text { to }-13 \\
9 \text { values }\end{array}$ & $\begin{array}{l}\text { Condition } \\
\text { Not } \\
\text { allowed }\end{array}$ & $\begin{array}{l}11.534896 \\
6 \text { to } 15 \\
10 \text { values }\end{array}$ & $\begin{array}{l}28.343229 \\
13 \text { to } 38 \\
26 \text { values }\end{array}$ \\
\hline $\mathrm{n}_{\mathrm{B}}=4$ & $\begin{array}{l}-28.115104 \\
-13 \text { to }-38 \\
26 \text { values }\end{array}$ & $\begin{array}{l}-23.306771 \\
-11 \text { to }-31 \\
21 \text { values }\end{array}$ & $\begin{array}{l}-14.498438 \\
-7 \text { to }-19 \\
13 \text { values }\end{array}$ & $\begin{array}{l}\text { Condition } \\
\text { Not } \\
\text { Allowed }\end{array}$ & $\begin{array}{l}15.118229 \\
7 \text { to } 20 \\
14 \text { values }\end{array}$ \\
\hline $\mathrm{n}_{\mathrm{B}}=5$ & $\begin{array}{l}-45.340104 \\
-21 \text { to }-38 \\
41 \text { values }\end{array}$ & $\begin{array}{l}-40.531771 \\
-19 \text { to }-55 \\
37 \text { values }\end{array}$ & $\begin{array}{l}-31.723438 \\
-15 \text { to }-43 \\
29 \text { values }\end{array}$ & $\begin{array}{l}-18.915104 \\
-9 \text { to }-25 \\
17 \text { values }\end{array}$ & $\begin{array}{l}-2.106771 \\
-1 \text { to }-2 \\
2 \text { values }\end{array}$ \\
\hline
\end{tabular}

Figure A.2

Precession Difference Functions for Halls B and C

\begin{tabular}{|c|c|c|c|c|c|}
\hline & $\mathrm{n}_{\mathrm{A}}=1$ & $\mathrm{n}_{\mathrm{A}}=2$ & $\mathrm{n}_{\mathrm{A}}=3$ & $\mathrm{n}_{\mathrm{A}}=4$ & $\mathrm{n}_{\mathrm{A}}=5$ \\
\hline $\mathrm{n}_{\mathrm{c}}=1$ & $\begin{array}{l}\text { Condition } \\
\text { Not } \\
\text { Allowed }\end{array}$ & $\begin{array}{l}6.521875 \\
3 \text { to } 8 \\
6 \text { values }\end{array}$ & $\begin{array}{l}16.163542 \\
8 \text { to } 22 \\
15 \text { values }\end{array}$ & $\begin{array}{l}29.805208 \\
14 \text { to } 40 \\
27 \text { values }\end{array}$ & $\begin{array}{l}47.446875 \\
22 \text { to } 64 \\
43 \text { values }\end{array}$ \\
\hline $\mathrm{n}_{\mathrm{c}}=2$ & $\begin{array}{l}-3.928125 \\
-2 \text { to }-5 \\
4 \text { values }\end{array}$ & $\begin{array}{l}\text { Condition } \\
\text { Not } \\
\text { Allowed }\end{array}$ & $\begin{array}{l}11.355208 \\
6 \text { to } 15 \\
10 \text { values }\end{array}$ & $\begin{array}{l}24.996875 \\
12 \text { to } 34 \\
23 \text { values }\end{array}$ & $\begin{array}{l}42.638542 \\
20 \text { to } 58 \\
39 \text { values }\end{array}$ \\
\hline $\mathrm{n}_{\mathrm{c}}=3$ & $\begin{array}{l}-12.736458 \\
-6 \text { to }-17 \\
12 \text { values }\end{array}$ & $\begin{array}{l}-7.094792 \\
-4 \text { to }-9 \\
6 \text { values }\end{array}$ & $\begin{array}{l}\text { Condition } \\
\text { Not } \\
\text { Allowed }\end{array}$ & $\begin{array}{l}16.188542 \\
8 \text { to } 22 \\
15 \text { values }\end{array}$ & $\begin{array}{l}33.830208 \\
16 \text { to } 46 \\
31 \text { values }\end{array}$ \\
\hline $\mathrm{n}_{\mathrm{c}}=4$ & $\begin{array}{l}-25.544792 \\
-12 \text { to }-34 \\
23 \text { values }\end{array}$ & $\begin{array}{l}-19.903125 \\
-10 \text { to }-27 \\
18 \text { values }\end{array}$ & $\begin{array}{l}-10.261458 \\
-5 \text { to }-13 \\
9 \text { values }\end{array}$ & $\begin{array}{l}\text { Condition } \\
\text { Not } \\
\text { Allowed }\end{array}$ & $\begin{array}{l}21.021875 \\
10 \text { to } 28 \\
19 \text { values }\end{array}$ \\
\hline $\mathrm{n}_{\mathrm{c}}=5$ & $\begin{array}{l}-42.353125 \\
-20 \text { to }-57 \\
38 \text { values }\end{array}$ & $\begin{array}{l}-36.711458 \\
-17 \text { to }-49 \\
24 \text { values }\end{array}$ & $\begin{array}{l}-27.069792 \\
-13 \text { to }-36 \\
24 \text { values }\end{array}$ & $\begin{array}{l}-13.428125 \\
-7 \text { to }-18 \\
12 \text { values }\end{array}$ & $\begin{array}{l}4.213542 \\
2 \text { to } 5 \\
4 \text { values }\end{array}$ \\
\hline
\end{tabular}

Figure A.3

Precession Difference Functions for Halls A and C 


\section{A.2 $\pi^{0}$ Asymmetry Data for All Runs}

Table A.1 shows the data used to calculate the $\pi^{0}$ asymmetries. The following are defined: run\# is the run number, $A_{\text {raw }}$ is the raw asymmetry, $P_{b} P_{t}$ is the product of the beam polarization and target polarization, $q^{+(-)}$is the charge for the positive (negative) helicity, $L^{+(-)}$is the livetime for positive (negative) helicity and $A_{p h y}$ is the corrected $\pi^{0}$ physics asymmetry. 
Table A. 1

$\pi^{0}$ Asymmetries for All Runs

\begin{tabular}{|c|c|c|c|c|c|c|c|}
\hline Run\# & $A_{\text {raw }}$ & $P_{b} P_{t}$ & $q^{+}$ & $q^{-}$ & $L^{+}$ & $L^{-}$ & $A_{p h y}$ \\
\hline 72417 & -0.0255 & -0.4855 & 64.36 & 64.57 & 0.8561 & 0.855 & -0.1111 \\
\hline 2421 & -0.0257 & -0.4478 & 111.66 & 112.05 & 0.847 & 0.8458 & -0.1202 \\
\hline 2422 & -0.021 & -0.4662 & 112.5 & 112.88 & 0.8451 & 0.8437 & -0.1134 \\
\hline 2423 & -0.0117 & -0.4574 & 110. & 110. & 0.8521 & 0.8509 & -0.1167 \\
\hline 2424 & -0.0378 & -0.4276 & 108.87 & 109.2 & 0.8516 & 0.8505 & -0.1242 \\
\hline 2428 & -0.0085 & -0.4456 & 116.03 & 116.42 & 0.8473 & 0.8463 & -0.1214 \\
\hline 2429 & -0.0105 & -0.4081 & 112. & 113.22 & & 0.8408 & -0.1329 \\
\hline 2430 & -0.0234 & -0.2962 & 22.28 & 22.36 & 0.8487 & 0.8477 & -0.1819 \\
\hline 2431 & -0.046 & -0.4001 & 95.47 & 95.78 & 0.8383 & 0.8371 & -0.1327 \\
\hline 2432 & -0.03 & -0.3844 & 107. & 107.4 & & & -0.1399 \\
\hline 2433 & -0.0099 & -0.3719 & 106.26 & 106.64 & 0.8493 & 0.8483 & -0.1484 \\
\hline 2434 & -0.0092 & -0.3611 & 106 & 106 & & 0.8469 & -0.1459 \\
\hline 2447 & -0.0042 & 0.3186 & 94.77 & 95.05 & 0.7959 & 0.7962 & 0.1829 \\
\hline 2448 & 0.0032 & 0.3875 & 86.67 & 86.94 & 0.8338 & 0.834 & 0.1508 \\
\hline 72449 & -0.00 & 0.3967 & 95. & 95 & & & 0.1468 \\
\hline 2450 & -0.02 & 0.3996 & 96.22 & 96. & & 0.8403 & \\
\hline 72477 & -0.0585 & 0.4972 & 85.3 & 85.43 & 0.8019 & 0.8016 & 0.103 \\
\hline 72479 & -0.01 & 0.51 & 81 & & & & 0.1007 \\
\hline 2480 & -0.01 & 0.5045 & 549.49 & 550.18 & 0.808 & 0.8085 & 0.1057 \\
\hline 72481 & -0.0062 & 0.4467 & 724.91 & 725.97 & 0.808 & 0.8083 & 0.1188 \\
\hline & & 0.45 & 470 & & & & \\
\hline 72483 & -0.0011 & 0.4638 & 760.6 & 761.74 & 0.8099 & 0.8101 & 0.1139 \\
\hline 72484 & 0.0091 & 0.461 & 748.62 & 749.62 & 0.8037 & 0.804 & 0.1139 \\
\hline 2485 & -0.01 & 0.4654 & 739 & 739.92 & 0.8 & 0.8082 & 0.11 \\
\hline 72488 & -0.0431 & 0.4674 & 5347.96 & 5348.72 & 0.7942 & 0.7943 & 0.1015 \\
\hline 2489 & -0.00 & & & & & & 017 \\
\hline 72491 & -0.0187 & 0.4368 & 5819 & 5820.33 & 0.8087 & 88 & 0.1094 \\
\hline 72492 & -0.0236 & 0.4284 & 2339.5 & 2339.68 & 0.8015 & 0.8017 & 0.1113 \\
\hline 72493 & 0.0093 & 0.4957 & 6040.08 & 6040.88 & 0.81 & & 0.0951 \\
\hline 72494 & -0.0037 & 0.4762 & 5840.51 & 5841.16 & 0.8143 & 0.8142 & 0.0969 \\
\hline 72495 & -0.0182 & 0.4672 & 5848.99 & 5849.85 & 0.8137 & 0.8139 & 0.1025 \\
\hline 72496 & -0.0028 & 0.4671 & 6029.87 & 6030.31 & 0.816 & 0.8161 & 0.1009 \\
\hline 72497 & -0.0389 & 0.4723 & 5972.93 & 5973.81 & 0.8143 & 0.8145 & 0.1013 \\
\hline 72498 & -0.0358 & 0.3386 & 1162.45 & 1162.59 & 0.8322 & 0.8323 & 0.1395 \\
\hline 72499 & -0.009 & 0.4792 & 6066.59 & 6067.69 & 0.8195 & 0.8198 & 0.1014 \\
\hline 72500 & -0.0295 & 0.4803 & 6062.35 & 6063.49 & 0.8209 & 0.8213 & 0.1017 \\
\hline
\end{tabular}


Table A.1

(continued)

\begin{tabular}{|c|c|c|c|c|c|c|c|}
\hline Run\# & $A_{\text {raw }}$ & $P_{b} P_{t}$ & $q^{+}$ & $q^{-}$ & $L^{+}$ & $L^{-}$ & $A_{p h y}$ \\
\hline & & 0.4683 & 6070.99 & 607160 & 0.8199 & 0.82 & \\
\hline 2502 & & & & & & & \\
\hline 2503 & & 1551 & & & & & 037 \\
\hline 2511 & & & 77 & & & & \\
\hline 2512 & & & & & & & -0.069 \\
\hline 2513 & -0.00 & & & & & & 12 \\
\hline & & & & & & & 76 \\
\hline 515 & & & & & & & \\
\hline 516 & & & & & & & -0.079 \\
\hline & & & & & & & \\
\hline & & & & & & & \\
\hline 519 & & & & & & & $-0 .($ \\
\hline 520 & & & & & & & \\
\hline 521 & & & & & & & -0 . \\
\hline 522 & & & & & & & -0 \\
\hline & & & & & & & \\
\hline 2524 & & & & & & & -0 . \\
\hline 2525 & & & & & & & \\
\hline & & & & & & & \\
\hline 527 & & & & & & & -0 . \\
\hline & & & & & & & \\
\hline & & & & & & & \\
\hline 2530 & & & & & & & -0.074 \\
\hline & & & & & & & \\
\hline & & & & & & & \\
\hline 2533 & -0.0 & & & & & & \\
\hline & & & & & & & \\
\hline 2546 & & & & & & & 0.0905 \\
\hline & & & & & & & \\
\hline & & & & & & & 0.095 \\
\hline & & & & & & & \\
\hline & & & & & & & \\
\hline & & & & & & & -0.087 \\
\hline & & & & & & & -0.104 \\
\hline 72553 & -0.0125 & -0.5035 & 6447.57 & 6448.56 & 0.8445 & 0.843 & -0.081 \\
\hline
\end{tabular}


Table A.1

(continued)

\begin{tabular}{|c|c|c|c|c|c|c|c|}
\hline Run\# & $A_{\text {raw }}$ & $P_{b} P_{t}$ & $q^{+}$ & $q^{-}$ & $L^{+}$ & $L^{-}$ & $A_{p h y}$ \\
\hline 72554 & 0.0029 & -0.5065 & 7096.27 & 7096.99 & 0.8452 & 0.8438 & -0.0812 \\
\hline 2555 & -0.0045 & -0.4763 & 4946.04 & 8 & 0.848 & 0.8467 & -0.0874 \\
\hline 2556 & -0.0114 & -0.296 & 2761.59 & 2761.88 & 0.8547 & 0.8536 & -0.1433 \\
\hline 2557 & 0.0051 & -0.4658 & 5559.58 & 5561.51 & 0.8056 & 0.8038 & -0.0848 \\
\hline 2559 & -0.0177 & -0.4997 & 4841.87 & 4842.76 & 0.8029 & 0.8012 & -0.0787 \\
\hline 2560 & -0.0149 & 0.4095 & 6784.92 & 6785.63 & 0.8449 & 0.845 & 0.1163 \\
\hline 2561 & 0.0027 & 0.4561 & 6732.8 & 6733 & 0.8481 & 0.8481 & 0.1033 \\
\hline 2564 & -0.0202 & 0.5651 & 6419.98 & 6421 & 0.8516 & 0.8516 & 0.0838 \\
\hline 2565 & 0123 & 0.5191 & 718 & 718 & 0.8482 & 0.8481 & 0.0896 \\
\hline 2566 & -0.0201 & 0.4764 & 7184.68 & 718 & 0.8505 & 0.8505 & 0.0982 \\
\hline 2567 & -0.0281 & 0.4591 & 7203.15 & 7204 & 0.8494 & 0.8494 & 0.1024 \\
\hline 2568 & & & & & & & \\
\hline 569 & & & 4192 & 419 & & & \\
\hline 2570 & -0.016 & 0.4246 & 4078.18 & 4078 & 0.8228 & 0.8227 & 0.1095 \\
\hline 2571 & -0.00 & & 7254 & 725 & & & \\
\hline 2572 & -0.0102 & 0.4852 & 737 & 737 & 0.8541 & 0.8541 & 0.0964 \\
\hline 2573 & -0.03 & 0.4715 & 743 & 743 & 44 & 47 & 019 \\
\hline & & & & & & & \\
\hline 72575 & -0.0238 & 0.4547 & 5994.77 & 5995 & 0.8571 & 0.8573 & 0.1055 \\
\hline 2576 & -0.0453 & 0.4546 & 5569.42 & $556 C$ & 558 & 557 & 016 \\
\hline 2577 & & 3 & 430 & 430 & & & 99 \\
\hline 2578 & -0.0188 & 0.4769 & 4465 & 446 & & & 977 \\
\hline & & & & & & & 19 \\
\hline 2580 & -0.0209 & 0.4338 & 2961. & 296 & & 07 & 111 \\
\hline 72581 & -0.0021 & 0.4637 & 4361.84 & 4362.37 & 0.8557 & 0.8558 & 0.1019 \\
\hline 72604 & -0.0472 & -0.5593 & & 32.32 & & & -0.0811 \\
\hline 72605 & -0.0544 & -0.5381 & & & & 68 & -0.0 \\
\hline 72606 & -0.0027 & -0.5277 & 33.52 & 33. & 0.5928 & 0.5915 & -0.0995 \\
\hline 72608 & -0.0015 & & & & & 0.8329 & -0.1053 \\
\hline 72609 & -0.0609 & 0.4962 & 66.35 & 66.48 & 0.8207 & 0.8212 & 0.1123 \\
\hline 72612 & -0.0271 & 0.4391 & 97.46 & & 0.8331 & 0.8329 & 0.1229 \\
\hline 72614 & -0.0023 & -0.5743 & & & 0.8312 & 0.8299 & -0.0922 \\
\hline 72615 & -0.0653 & -0.5532 & 46.59 & 46.77 & 0.8279 & 0.8264 & -0.0969 \\
\hline 72616 & -0.0303 & -0.546 & 17.83 & & & 0.8958 & -0.1119 \\
\hline 72617 & -0.0599 & -0.5685 & 25.02 & & 0.8949 & 0.8939 & -0.107 \\
\hline 72618 & -0.0074 & 0.5381 & 48.86 & 48.95 & 0.829 & 0.8292 & 0.1002 \\
\hline
\end{tabular}


Table A.1

(continued)

\begin{tabular}{|c|c|c|c|c|c|c|c|}
\hline Run\# & $A_{\text {raw }}$ & $P_{b} P_{t}$ & $q^{+}$ & $q^{-}$ & $L^{+}$ & $L^{-}$ & $A_{p h y}$ \\
\hline 2619 & -0.0009 & 0.5094 & 48.35 & 48.45 & 0.8301 & 08305 & 01007 \\
\hline 620 & & & & & & & \\
\hline 621 & & & 47.63 & 47.75 & & & \\
\hline & & & & & & & \\
\hline 2631 & -0.01 & -0.4357 & 67.44 & 67. & 38 & 8125 & 0.1152 \\
\hline 632 & & -0.4 & & & & 0.0101 & $17^{7}$ \\
\hline 633 & & & & & & & \\
\hline 63 & & & & & & & \\
\hline & & & & & & & \\
\hline 636 & & & & & & & 028 \\
\hline 637 & & & & & & & 10 \\
\hline & & & & & & & \\
\hline $62+2$ & & & & & & & 213 \\
\hline 640 & & & & & & & -0.1252 \\
\hline & & & & & & & \\
\hline 642 & & & & & & & -0.125 \\
\hline 645 & & & & & & & 097 \\
\hline & & & & & & & \\
\hline 647 & & & & & & & 27 \\
\hline 649 & & & & & & & 34 \\
\hline & & & & & & & \\
\hline & & & & & & & 008 \\
\hline & & & & & & & \\
\hline & & & & & & & \\
\hline 2661 & -0.0 & 654 & & 05 & & & .0979 \\
\hline & & & & & & & \\
\hline & & & & & & & 017 \\
\hline & & & & & & & \\
\hline & & & & & & & 1081 \\
\hline & & & & & & & 0.1094 \\
\hline & & & & & & & 0.1103 \\
\hline & & & & & & & 0.1093 \\
\hline & & & & & & & 0.1051 \\
\hline 72670 & 0.0089 & 0.519 & 74.94 & 75.13 & 0.8527 & 0.8532 & 0.1113 \\
\hline
\end{tabular}


Table A.1

(continued)

\begin{tabular}{|c|c|c|c|c|c|c|c|}
\hline Run\# & $A_{\text {raw }}$ & $P_{b} P_{t}$ & $q^{+}$ & $q^{-}$ & $L^{+}$ & $L^{-}$ & $A_{p h y}$ \\
\hline 2671 & -0.0119 & 0.4949 & & & 0.8188 & 0.8195 & \\
\hline 672 & & & & & & & \\
\hline & & & & & & & \\
\hline 674 & & & & & & & \\
\hline & & & & & & & \\
\hline 681 & & 82 & 32.2 & 32. & & & \\
\hline 682 & 79 & -0.6 & 61. & 62 & & & \\
\hline & & & & & & & \\
\hline 684 & -0.0 & & & 76 & & & \\
\hline 685 & & & & & & & 21 \\
\hline & & & & & & & \\
\hline 687 & & & 77. & & & & \\
\hline & & & & & & & \\
\hline 0 & & & & & & & \\
\hline 692 & & & & & & & \\
\hline & & & & & & & \\
\hline & & & & & & & \\
\hline & & & & & & & \\
\hline & & & & & & & \\
\hline 703 & & & & & & & \\
\hline 708 & & & & & & & \\
\hline & & & & & & & \\
\hline & & & & & & & \\
\hline 2711 & & & & & & & \\
\hline & & & & & & & \\
\hline 713 & & & & & & & \\
\hline & & & & & & & \\
\hline & & & & & & & 427 \\
\hline 2716 & -0.03 & 0.3108 & & & & & \\
\hline & & & & & & & \\
\hline 0726 & & & & & & & 0.1237 \\
\hline & & & & & & & \\
\hline & & & & & & & \\
\hline & & & & & & & 0.1381 \\
\hline 72743 & 0.0043 & 0.4801 & 155.3 & 155.97 & 0.8352 & 0.8356 & 0.1319 \\
\hline
\end{tabular}


Table A.1

(continued)

\begin{tabular}{|c|c|c|c|c|c|c|c|}
\hline Run\# & $A_{\text {raw }}$ & $P_{b} P_{t}$ & $q^{+}$ & $q^{-}$ & $L^{+}$ & $L^{-}$ & $A_{p h y}$ \\
\hline 2744 & & 0.4721 & 153.6 & 154.17 & 0.8368 & 0.8369 & 01260 \\
\hline 745 & & 383 & & & & & 1498 \\
\hline 746 & 9 & 0.4547 & & & & & .1348 \\
\hline 747 & & & & & & & \\
\hline 748 & & 0.4429 & & & & & \\
\hline 749 & & & & & & & \\
\hline 750 & & 79 & & & & & 151 \\
\hline 751 & 3 & 3675 & 87.43 & & & & 577 \\
\hline 752 & & & & & & & \\
\hline 755 & & & & & & & 951 \\
\hline 756 & & & & & & & -0.092 \\
\hline 759 & & & & & & & \\
\hline 761 & & & & & & & 968 \\
\hline 763 & & & & & & & \\
\hline & & & & & & & \\
\hline 765 & & & & & & & 26 \\
\hline 769 & & & & & & & \\
\hline 770 & & & & & & & 972 \\
\hline 771 & & & & & & & \\
\hline & & & & & & & \\
\hline 773 & & & & & & & 59 \\
\hline 774 & & & & & & & 088 \\
\hline & & & & & & & 052 \\
\hline 776 & & & & & & 0.7744 & 127 \\
\hline & & & & & & & \\
\hline & & & & & & & \\
\hline 779 & -0.0497 & .455 & 39.47 & & 0.8475 & 0.8461 & -0.1181 \\
\hline & & & & & & & \\
\hline 2706 & & & & & & & 1347 \\
\hline & & & & & & & 0.1325 \\
\hline & & & & & & & 0.13 \\
\hline & & & & & & & 0.1394 \\
\hline & & & & & & & \\
\hline 72794 & -0.0365 & 0.4761 & 36.27 & 36.42 & 0.8465 & 0.8465 & 0.1275 \\
\hline
\end{tabular}


Table A.1

(continued)

\begin{tabular}{|c|c|c|c|c|c|c|c|}
\hline Run\# & $A_{\text {raw }}$ & $P_{b} P_{t}$ & $q^{+}$ & $q^{-}$ & $L^{+}$ & $L^{-}$ & $A_{p h y}$ \\
\hline 72795 & & 0.5001 & 151.88 & 152.5 & 08375 & 08274 & 0.1218 \\
\hline & & & & & & & \\
\hline 797 & & 0.4663 & & & & & \\
\hline & & & & & & & \\
\hline 799 & 13 & 447 & & 2 & 8179 & & 1302 \\
\hline 824 & & -0.7764 & & & & & 58 \\
\hline & & & & & & & \\
\hline 826 & & & & & & & \\
\hline & & & & & & & \\
\hline 828 & & & & & & & \\
\hline 829 & 7 & & & & & & 72 \\
\hline & & & & & & & \\
\hline & & & & & & & \\
\hline 47 & & & & & & & \\
\hline & & & & & & & \\
\hline & & & & & & & \\
\hline & & & & & & & \\
\hline & & & & & & & \\
\hline & & & & & & & \\
\hline & & & & & & & \\
\hline & & & & & & & \\
\hline & & & & & & & \\
\hline & & & & & & & \\
\hline & & & & & & & \\
\hline 875 & & -0.6457 & 124 & 9 & 34 & & \\
\hline & & & & & & & \\
\hline & & & & & & & \\
\hline & & & & & & & \\
\hline & & & & & & & \\
\hline & & & & & & & \\
\hline & & & & & & & \\
\hline & & & & & & 0.8384 & -0.0652 \\
\hline & & & & & & & \\
\hline 72916 & -0.0155 & -0.5356 & 177.51 & 177.88 & 0.9109 & 0.9095 & -0.0904 \\
\hline
\end{tabular}


Table A.1

(continued)

\begin{tabular}{|c|c|c|c|c|c|c|c|}
\hline Run\# & $A_{\text {raw }}$ & $P_{b} P_{t}$ & $q^{+}$ & $q^{-}$ & $L^{+}$ & $L^{-}$ & $A_{p h y}$ \\
\hline 72917 & & -0.5098 & & 14470 & 0.912 & 0.911 & \\
\hline & & & & & & & \\
\hline 919 & & & & & & & \\
\hline & & & & & & & \\
\hline 922 & & -0.4335 & 89. & & 912 & & \\
\hline 923 & & & 12 & & & & \\
\hline & & & & & & & \\
\hline & & & & & & & \\
\hline & & & & & & & \\
\hline 929 & & & & & & & \\
\hline 931 & & & & & & & \\
\hline & & & & & & & \\
\hline & & & & & & & \\
\hline & & & & & & & \\
\hline & & & & & & & \\
\hline & & & & & & & \\
\hline 941 & & & & & & & \\
\hline & & & & & & & \\
\hline 943 & & & & & & & \\
\hline 944 & & & & & & & \\
\hline & & & & & & & \\
\hline 946 & & & & & & & \\
\hline & & & & & & & \\
\hline & & & & & & & \\
\hline 2950 & & $-0.4 \varepsilon$ & 157. & 32 & .9139 & & \\
\hline & & & & & & & \\
\hline & & & & & & & \\
\hline & & & & & & & \\
\hline & & & & & & & \\
\hline & & & & & & & \\
\hline & & & & & & & \\
\hline & & & & & & & 0.1263 \\
\hline & & & & & & & \\
\hline 72992 & -0.0047 & 0.4115 & 281.16 & 281.16 & 0.9046 & 0.9059 & 0.1255 \\
\hline
\end{tabular}


Table A.1

(continued)

\begin{tabular}{cccccccc}
\hline Run\# & $A_{\text {raw }}$ & $P_{b} P_{t}$ & $q^{+}$ & $q^{-}$ & $L^{+}$ & $L^{-}$ & $A_{\text {phy }}$ \\
\hline 72999 & -0.0395 & 0.4538 & 160.33 & 160.24 & 0.9416 & 0.943 & 0.1088 \\
73001 & -0.0486 & 0.3865 & 160.64 & 160.57 & 0.9331 & 0.9343 & 0.1278 \\
73002 & -0.0418 & -0.4951 & 208.35 & 208.46 & 0.942 & 0.9405 & -0.0865 \\
73003 & -0.0274 & -0.4604 & 192.69 & 192.89 & 0.9417 & 0.9403 & -0.0975 \\
73004 & 0.0182 & -0.4253 & 255.18 & 255.39 & 0.9409 & 0.9396 & -0.1055 \\
73005 & -0.002 & -0.4198 & 297.37 & 297.75 & 0.9339 & 0.9326 & -0.1099 \\
73010 & -0.018 & 0.3977 & 50.25 & 50.32 & 0.9382 & 0.9397 & 0.144 \\
73012 & -0.0284 & -0.4181 & 385.37 & 384.97 & 0.9392 & 0.938 & -0.0918 \\
73013 & -0.0253 & -0.5783 & 253.85 & 253.82 & 0.9465 & 0.9453 & -0.0719 \\
73014 & -0.0361 & -0.4818 & 163.19 & 163.19 & 0.9419 & 0.9406 & -0.0862 \\
73015 & -0.0187 & -0.4682 & 195.25 & 195.18 & 0.9495 & 0.9487 & -0.09 \\
73017 & -0.0292 & -0.4159 & 140.89 & 140.83 & 0.9426 & 0.9414 & -0.0976 \\
73018 & -0.0499 & -0.4053 & 83.03 & 83.04 & 0.9342 & 0.9331 & -0.1046 \\
73019 & -0.04 & -0.4348 & 256.51 & 256.41 & 0.944 & 0.9428 & -0.0934 \\
73020 & 0.001 & -0.3921 & 246.79 & 246.72 & 0.9443 & 0.9433 & -0.1058 \\
73021 & -0.0905 & -0.3675 & 65.17 & 65.17 & 0.9333 & 0.9319 & -0.1135 \\
73028 & -0.0039 & 0.4201 & 173.62 & 173.61 & 0.9421 & 0.9431 & 0.1197 \\
73029 & 0.0369 & 0.3757 & 41.87 & 41.88 & 0.9295 & 0.9308 & 0.1394 \\
73031 & 0.011 & -0.5139 & 162.97 & 163 & 0.9431 & 0.9417 & -0.0815 \\
73035 & 0.0078 & -0.4568 & 198.3 & 198.33 & 0.9459 & 0.9448 & -0.0935 \\
73036 & -0.0344 & -0.4383 & 149.01 & 149.07 & 0.9415 & 0.9403 & -0.0993 \\
73037 & -0.031 & -0.4259 & 155.31 & 155.37 & 0.9416 & 0.9406 & -0.1036 \\
\hline & & & & & & &
\end{tabular}

